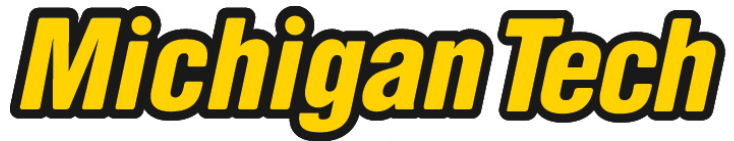 \\ Michigan Technological University Create the Future Digital Commons @ Michigan Tech
}

Dissertations, Master's Theses and Master's Reports - Open

Dissertations, Master's Theses and Master's

Reports

2006

Study of and design procedure for dual circularly polarized waveguide slot arrays

Lee M. Paulsen

Michigan Technological University

Follow this and additional works at: https://digitalcommons.mtu.edu/etds

Part of the Electrical and Computer Engineering Commons

Copyright 2006 Lee M. Paulsen

\section{Recommended Citation}

Paulsen, Lee M., "Study of and design procedure for dual circularly polarized waveguide slot arrays", Dissertation, Michigan Technological University, 2006.

https://doi.org/10.37099/mtu.dc.etds/70

Follow this and additional works at: https://digitalcommons.mtu.edu/etds

Part of the Electrical and Computer Engineering Commons 


\title{
A Study of and Design Procedure for Dual Circularly Polarized Waveguide Slot Arrays
}

\author{
By \\ Lee M Paulsen \\ A DISSERTATION \\ Submitted in partial fulfillment of the requirements \\ for the degree of \\ DOCTOR OF PHILOSOPHY \\ Electrical and Computer Engineering \\ MICHIGAN TECHNOLOGICAL UNIVERSITY \\ 2006
}


Copyright (C) Lee M Paulsen 2006 
This dissertation, "A Study of and Design Procedure for Dual Circularly Polarized Waveguide Slot Arrays," is hereby approved in partial fulfillment of the requirements for the degree of DOCTOR OF PHILOSOPHY in the field of Electrical and Computer Engineering.

DEPARTMENT or PROGRAM:

Electrical and Computer Engineering

Signatures:

Dissertation Advisor Dr. Warren Perger

Committee

Dr. Jon Soper

Dr. Paul Bergstrom

Dr. Barbara Bertram

Department Chair Dr. Timothy Schulz

Date 


\section{Abstract}

For the past sixty years, waveguide slot radiator arrays have played a critical role in microwave radar and communication systems. They feature a wellcharacterized antenna element capable of direct integration into a low-loss feed structure with highly developed and inexpensive manufacturing processes. Waveguide slot radiators comprise some of the highest performance - in terms of side-lobe-level, efficiency, etc. - antenna arrays ever constructed.

A wealth of information is available in the open literature regarding design procedures for linearly polarized waveguide slots. By contrast, despite their presence in some of the earliest published reports, little has been presented to date on array designs for circularly polarized (CP) waveguide slots. Moreover, that which has been presented features a classic traveling wave, efficiency-reducing beam tilt. This work proposes a unique $\mathrm{CP}$ waveguide slot architecture which mitigates these problems and a thorough design procedure employing widely available, modern computational tools.

The proposed array topology features simultaneous dual-CP operation with grating-lobe-free, broadside radiation, high aperture efficiency, and good return loss. A traditional X-Slot $\mathrm{CP}$ element is employed with the inclusion of a slow wave structure passive phase shifter to ensure broadside radiation without the need for performance-limiting dielectric loading. It is anticipated this technology will be advantageous for upcoming polarimetric radar and Ka-band SatCom systems.

The presented design methodology represents a philosophical shift away from traditional waveguide slot radiator design practices. Rather than providing design curves and/or analytical expressions for equivalent circuit models, simple first-order design rules - generated via parametric studies — are presented with the understanding that device optimization and design will be carried out computationally. A unit-cell, S-parameter based approach provides a sufficient reduction of complexity to permit efficient, accurate device design with attention to realistic, application-specific mechanical tolerances. 
A transparent, start-to-finish example of the design procedure for a linear sub-array at X-Band is presented. Both unit cell and array performance is calculated via finite element method simulations. Results are confirmed via good agreement with finite difference, time domain calculations. Array performance exhibiting grating-lobe-free, broadside-scanned, dual-CP radiation with better than $20 \mathrm{~dB}$ return loss and over $75 \%$ aperture efficiency is presented. 


\section{Acknowledgements}

I would like to acknowledge and thank several people who have made a positive impression on this work. First, I'd like to thank Dr. Warren Perger for his steadfast optimism and the support he gave me throughout this long process. Ours was perhaps an unorthodox student-advisor relationship as I was never able to take any of his classes and we interacted very little on a technical level, but I consider his direction and advice invaluable. I would like to thank him along with Drs. Paul Bergstrom, Jon Soper, and Mark Gockenbach for sacrificing the time to read through my dissertation. Their thoughtful comments and suggestions strengthened it. Also, I would be remiss to not acknowledge the great service Dr. Barb Bertram did for me, stepping in at the last minute to fill a sudden vacancy on my committee.

I would like to acknowledge and thank my colleagues at Rockwell Collins. The fascinating conversations we've had on slot radiators, as well as, the insightful and challenging questions you posed over the last year-and-a-half helped me greatly. From this group I would like to especially acknowledge the help of my manager, Jim West. He first introduced me to waveguide slot radiators and in many ways helped guide me more than anyone else. I deeply appreciate the always cheerful suggestions he made when the work stalled, as well as his contagious enthusiasm for all things electromagnetic. Although we openly disagreed on several aspects of this work (I told you an equivalent circuit wasn't necessary!), his keen intellect and wealth of experience greatly strengthened its quality.

Finally, I would like to thank my wife and one true love, Kim. Without your continual support this research would never have been completed. The depth of your sacrifices is perhaps something few others will ever know, but know that they are appreciated at a level of expression beyond my feeble grasp of our language. The countless nights I spent working on this while you watched our first child, Isabelle, or simply waited patiently for me to finish are time I can never return to you. Thank you. I love you. 


\section{Table of Contents}

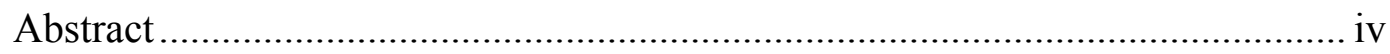

Acknowledgements....................................................................................... vi

Table of Figures ..................................................................................... $\mathrm{x}$

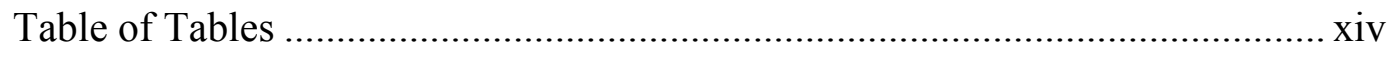

Chapter 1: Introduction and Historical Setting ................................................... 1

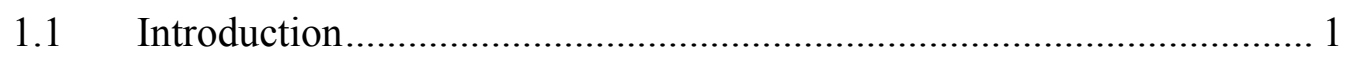

$1.2 \quad$ Linearly Polarized Slots ................................................................... 2

$1.3 \quad$ Circularly Polarized Slots ...................................................................... 3

1.4 Overview and Goals of Work ……………………………………..... 4

Chapter 2: The Problems and a Proposed Solution ................................................... 7

2.1 Dual Circular Polarization ................................................................ 7

2.2 The Spatial Beam Divergence Problem................................................. 9

2.3 The Grating Lobe Problem .................................................................... 10

2.4 Dielectric Loading and the G/T Problem................................................ 11

2.5 The Design Problem …………………………………..................... 13

2.6 A Solution Approach ................................................................... 15

Chapter 3: Array Topology and Design Approach ............................................... 17

3.1 The Array Topology ........................................................................... 17

3.1.1 The Radiating Elements ................................................................. 18

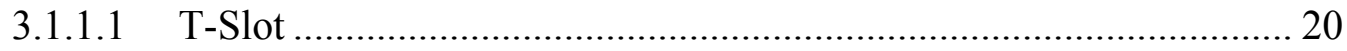

3.1.1.2 Offset Compound Slot Pair ........................................................... 26

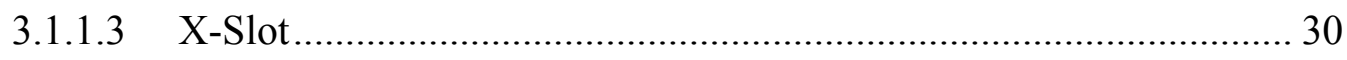

3.1.1.4 Radiator Down Selection ................................................................ 36

3.1.2 The Phase Shifter ........................................................................ 37

3.1.2.1 Phase Shifter Loss..................................................................... 38

3.1.2.2 Design Challenges ……………………….................................... 40

3.1.2.3 Phase Shifter Down Selection.......................................................... 42

3.2 The Design Approach ………………………................................... 42 


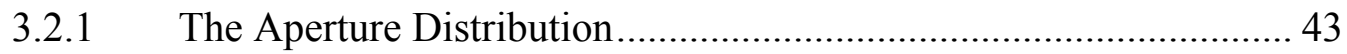

Chapter 4: Slow Wave Structure Passive Phase Shifters...................................... 48

4.1 Introduction and Definition................................................................ 48

4.2 Slow Wave Structures and Waveguide Slot Arrays ............................. 50

4.3 Slow Wave Structure Analysis …………………………………......... 50

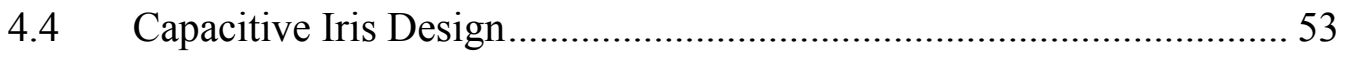

4.5 Slow Wave Structure Design ................................................................. 55

4.6 Unoptimized Slow Wave Structure Return Loss ................................... 59

4.7 Optimized Slow Wave Structure Return Loss ......................................... 61

4.7.1 Optimized Slow Wave Structure Return Loss .................................... 61

4.7.2 Re-Optimized Slow Wave Structure Return Loss .............................. 65

4.8 A Discrete Slow Wave Structure Design Methodology ........................ 67

Chapter 5: X-Slot Parametric Study and Design Rules ............................................ 69

5.1 A Discrete Slow Wave Structure Design Methodology ......................... 69

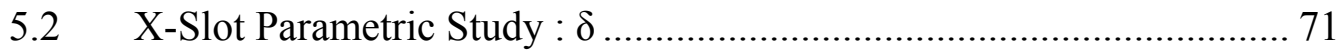

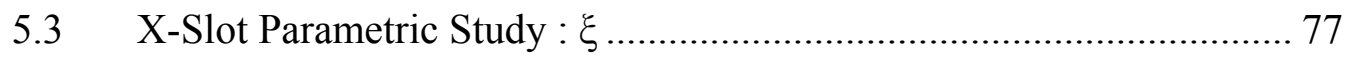

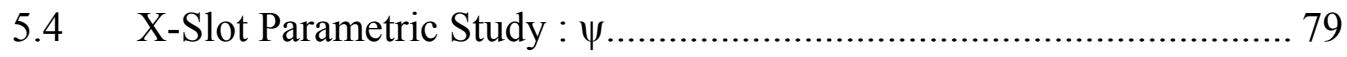

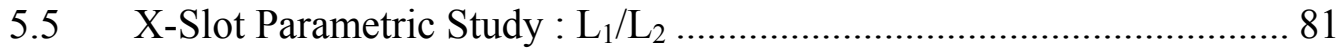

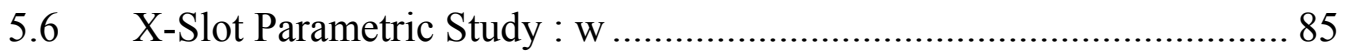

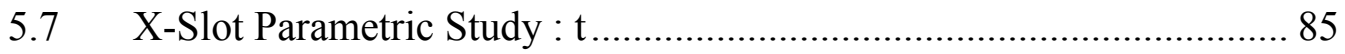

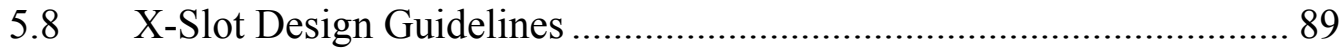

Chapter 6: Array Design Methodology and Example............................................ 92

6.1 Dual CP Linear Array Design Methodology ………………………...... 92

6.2 Dual CP Broadside Radiating X-Slot Array Design Example............... 94

6.2.1 Step 1: Array Layout................................................................... 95

6.2.2 Step 2 : Slot Coupling Ratio ........................................................... 96

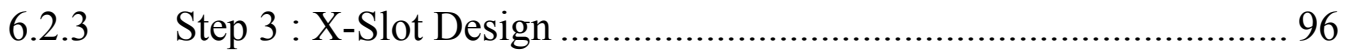

6.2.4 Step 4: Slow Wave Structure Design................................................... 99

6.2.5 Step 5 : Simulate the Linear Array .................................................. 104

6.2.5.1 The HFSS Model ……………………….................................. 104

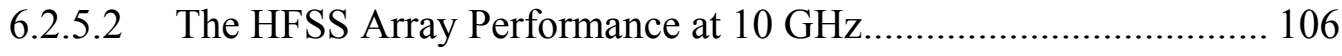


6.2.5.3 The HFSS Array Performance from 9.5-10.5 GHz ....................... 109

6.2.5.4 The CST Comparison Model ........................................................ 115

6.2.5.5 CST vs. HFSS : Radiation Patterns.................................................. 115

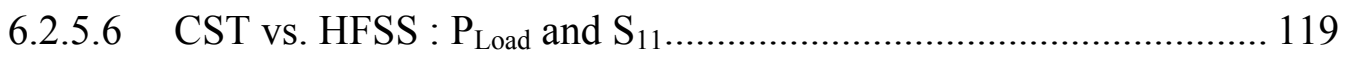

6.3 Array Design Example Conclusions................................................... 121

Chapter 7: Conclusions and Future Work......................................................... 124

7.1 Overview and Conclusions .............................................................. 124

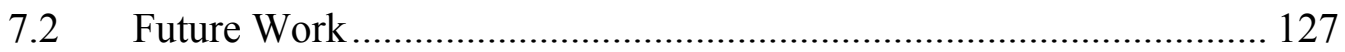

Appendix A : An Alternate X-Slot Theory of Operation ................................... 128

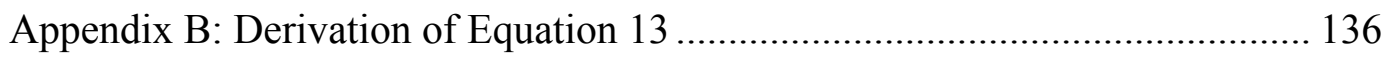

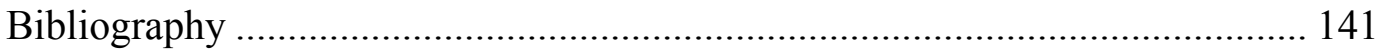




\section{Table of Figures}

Figure 1 : Linearly Polarized Waveguide Slots .................................................. 1

Figure 2 : Circularly Polarized Waveguide Slot Combinations............................... 3

Figure 3 : Typical Dual CP Waveguide Slot Array .............................................. 8

Figure 4 : Typical Dual CP Waveguide Slot Array Gain Pattern ........................... 8

Figure 5 : Proposed Dual CP Array Topology .................................................... 15

Figure 6 : Proposed Linear Subarray .............................................................. 17

Figure 7 : Rectangular Waveguide Broad Wall ................................................... 18

Figure $8: \mathrm{TE}_{10}$ Notional Magnetic Field Distribution............................................ 19

Figure 9: $\mathrm{TE}_{10}$ Magnetic Field Vector Plot........................................................ 20

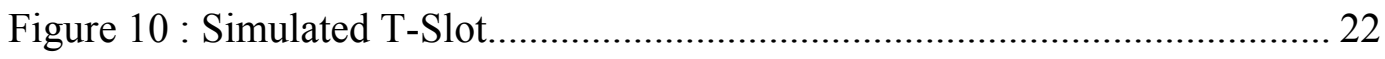

Figure 11 : T-Slot LHCP Pattern ........................................................................ 22

Figure 12 : T-Slot RHCP Pattern .......................................................................... 23

Figure 13 : T-Slot Half Space CoPol Gain (dB) ……………………………... 24

Figure 14 : T-Slot Half Space XPol Gain (dB)............................................... 25

Figure 15 : T-Slot S-Parameter Performance...................................................... 26

Figure 16 : Simulated Offset Compound Slot Pair …………………………..... 27

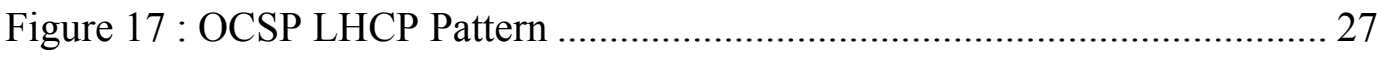

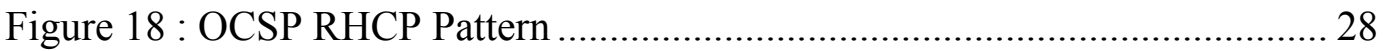

Figure 19: OCSP Half Space CoPol Gain (dB) ............................................... 29

Figure 20 : OCSP Half Space XPol Gain (dB) ……………………................. 29

Figure 21 : OCSP S-Parameter Performance..................................................... 30

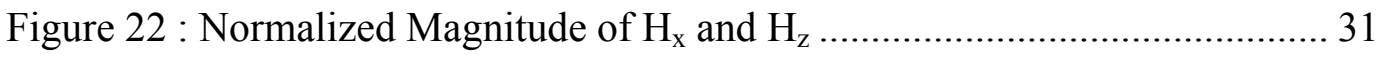

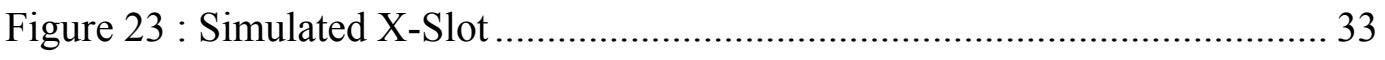

Figure 24 : X-Slot LHCP Pattern........................................................................ 33

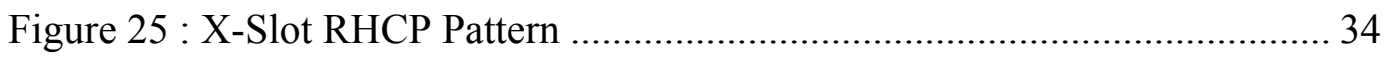

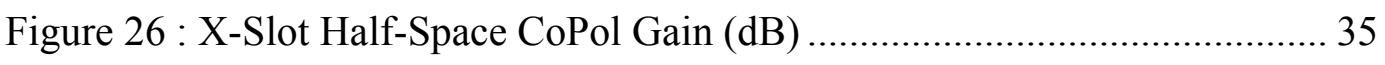

Figure 27 : X-Slot Half-Space XPol Gain (dB) ................................................... 35

Figure 28 : X-Slot S-Parameter Performance …………………………............... 36

Figure 29 : Passive Waveguide Phase Shifters ..................................................... 38 
Figure 30 : Traveling Wave 15-Element Linear Array Slot Conductance ........... 44

Figure 31 : Traveling Wave Linear Array Aperture Power Distribution.............. 45

Figure 32 : Fwd and Rev Fed Traveling Wave Linear Array Directivities .......... 45

Figure 33 : TW Aperture Efficiency Comparison .............................................. 46

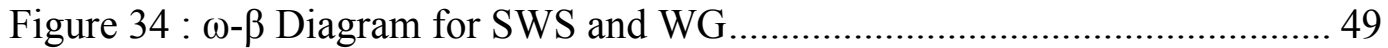

Figure 35 : Capacitive Iris Slow Wave Structure ............................................. 51

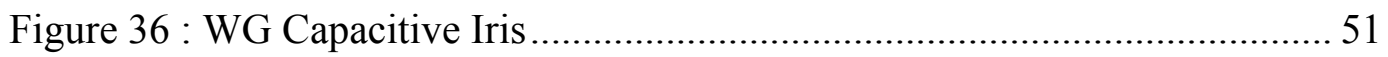

Figure 37 : WG Capacitive Iris Equiv Circuit ................................................. 51

Figure 38 : Arbitary Terminated T-network ............................................... 52

Figure 39 : Theoretical vs. Simulated Iris Susceptance.................................... 54

Figure 40: Discrete Slow Wave Structure ....................................................... 55

Figure $41: \mathrm{N}=3 \mathrm{SWS}$ from Theoretical Expressions ….................................. 56

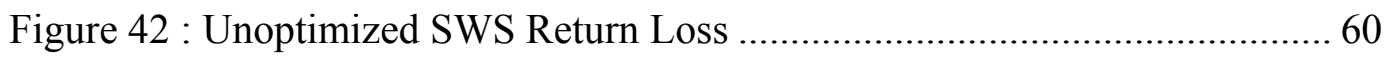

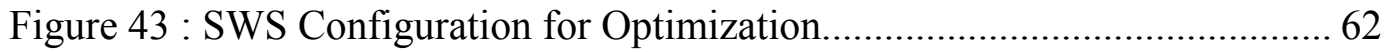

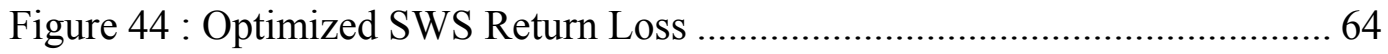

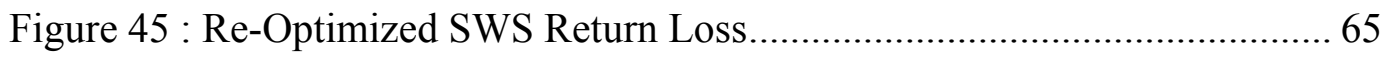

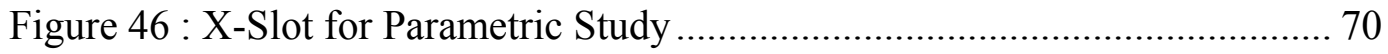

Figure $47: 8 \mathrm{~mm}$ X-Slot, $12.5 \mathrm{GHz}$ Radiation Performance vs. $\delta$..................... 72

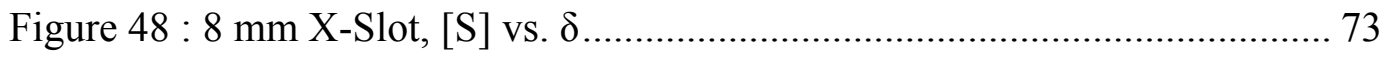

Figure 49 : 11.5 mm X-Slot, $12.5 \mathrm{GHz}$ Radiation Performance vs. $\delta$................. 74

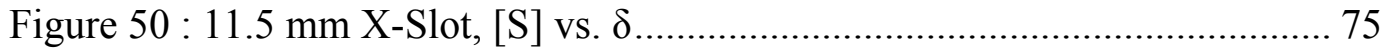

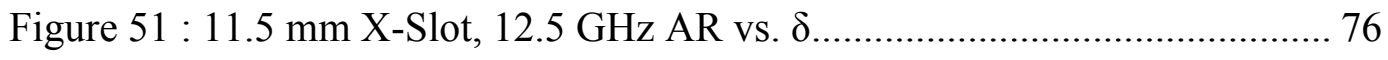

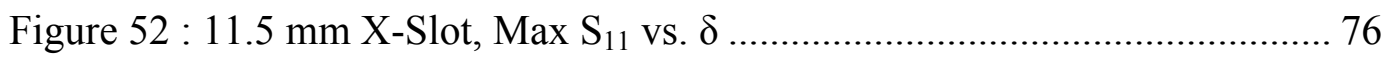

Figure 53 : $11.5 \mathrm{~mm}$ X-Slot, $12.5 \mathrm{GHz}$ Radiation Performance vs. $\xi$................. 78

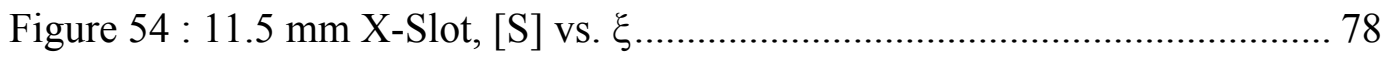

Figure 55 : $11.5 \mathrm{~mm}$ X-Slot, $12.5 \mathrm{GHz}$ Radiation Performance vs. $\psi$................ 80

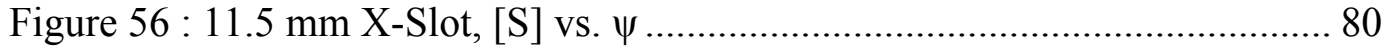

Figure 57 : $11.5 \mathrm{~mm}$ X-Slot, $12.5 \mathrm{GHz}$ Radiation Performance vs. $\mathrm{L}_{1} / \mathrm{L}_{2} \ldots \ldots \ldots . . .82$

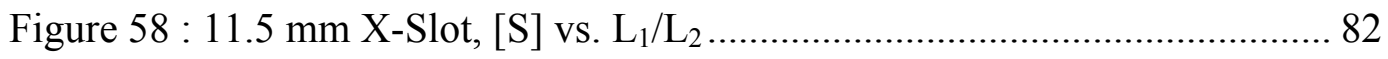

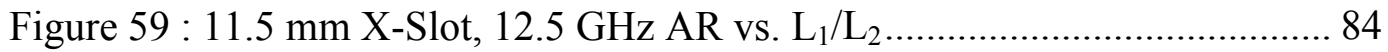

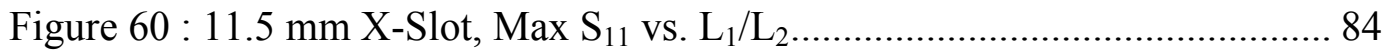


Figure 61 : $11.5 \mathrm{~mm}$ X-Slot, $12.5 \mathrm{GHz}$ Radiation Performance vs. t.................. 87

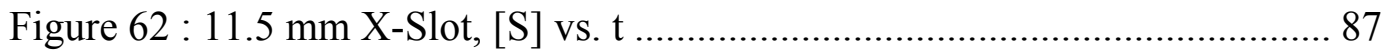

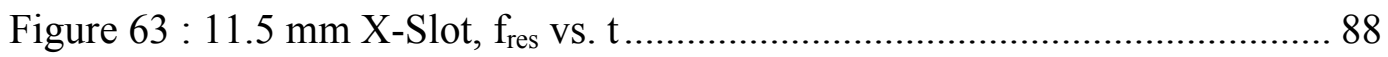

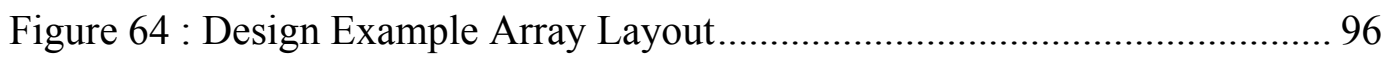

Figure 65 : Initial X-Slot, L = 14 mm, S-Parameters...................................... 97

Figure 66 : Optimized X-Slot, L = 14.2 mm, S-Parameters ............................. 98

Figure 67 : Optimized X-Slot, $\mathrm{L}=14.2$ mm, Radiation Patterns ........................ 99

Figure 68 : Initial SWS Return Loss........................................................... 100

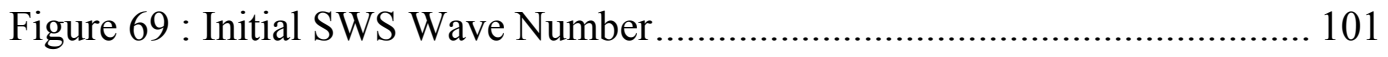

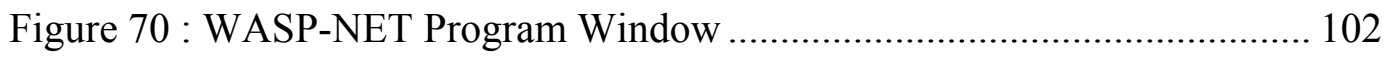

Figure 71 : Optimized SWS Return Loss ..................................................... 103

Figure 72 : Optimized SWS Wave Number .................................................. 103

Figure 73 : HFSS Linear Array Design Example Model................................. 105

Figure 74 : Linear Array, Port 1 Radiation Patterns ........................................ 106

Figure 75 : Linear Array, Port 2 Radiation Patterns ........................................ 107

Figure 76 : $10 \mathrm{GHz}$ Linear Array CoPol Gain (dB)..................................... 108

Figure 77 : 10 GHz Linear Array XPol Gain (dB) ...................................... 108

Figure 78 : Linear Array Gain at Zenith vs. Frequency.................................. 110

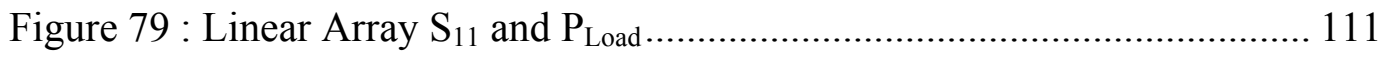

Figure 80 : Linear Array Main Beam Direction vs. Frequency ......................... 112

Figure 81 : 9.5 GHz Linear Array CoPol Gain (dB)..................................... 113

Figure 82 : 10.5 GHz Linear Array CoPol Gain (dB).................................. 113

Figure 83 : Linear Array Boresite and Peak Gain vs. Frequency ...................... 114

Figure 84 : CST Linear Array Design Example Model................................... 115

Figure 85 : Linear Array 10 GHz Directivity, HFSS vs. CST .......................... 117

Figure 86 : Linear Array 10 GHz Main Beam Comparison, HFSS vs. CST ...... 117

Figure 87 : CST Linear Array $10 \mathrm{GHz}$ CoPol Directivity (dB)....................... 118

Figure 88 : CST Linear Array 9.5 GHz CoPol Directivity $(\mathrm{dB})$....................... 118

Figure 89 : CST Linear Array 10.5 GHz CoPol Directivity (dB)..................... 119

Figure 90 : Linear Array $\mathrm{S}_{11}$ HFSS vs. CST .................................................. 120

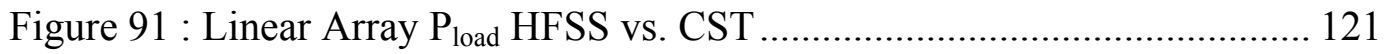


Figure 92 : Compound Slot Study Unit Cell............................................. 128

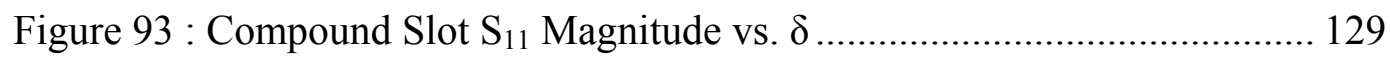

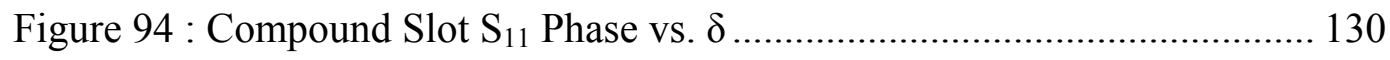

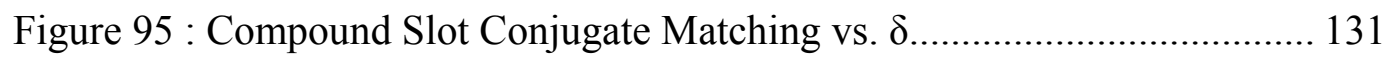

Figure 96 : Compound Slot Aperture Field Magnitude Comparison ................. 132

Figure 97 : Compound Slot Aperture Field Phase Comparison ........................ 132

Figure 98 : Double Crescent Slot Unit Cell ................................................. 133

Figure 99 : DCS Unit Cell S-Parameter Performance ..................................... 134

Figure 100 : DCS Unit Cell Radiation Performance ....................................... 134 


\section{Table of Tables}

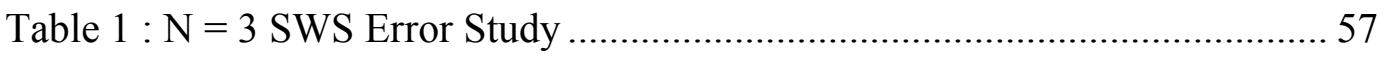

Table 2 : SWS B Study - HFSS vs. Collin....................................................... 58

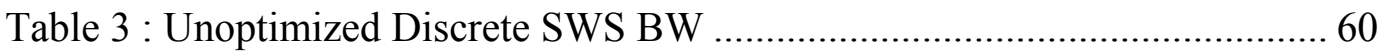

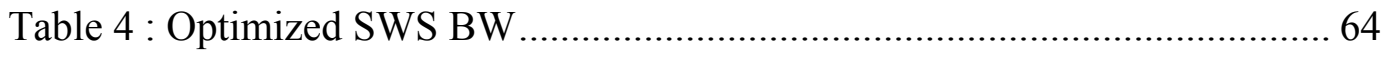

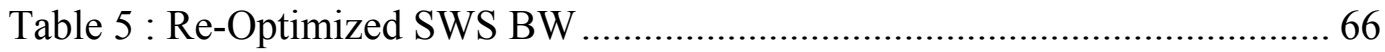

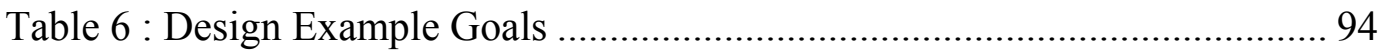

Table 7 : Design Example Array Layout ......................................................... 95

Table 8 : Linear Array Simulated S-Parameter and Gain Data ......................... 109 


\section{Chapter 1: Introduction and Historical Setting}

\subsection{Introduction}

A waveguide slot radiator is fundamentally any aperture intentionally placed in a waveguide wall. Most commonly these apertures are located in the broad or narrow walls, but they may also be located in a shorting plate placed across the guide cross section. A myriad of shapes are theoretically possible, however, only a select few have been well characterized and are commonly used. The linearly polarized (LP) waveguide slots depicted in Figure 1 have formed the bulk of the waveguide slot radiator publication canon.

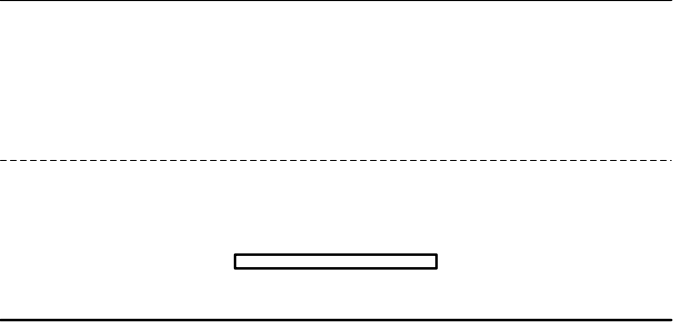

(a) Longitudinal Shunt Slot

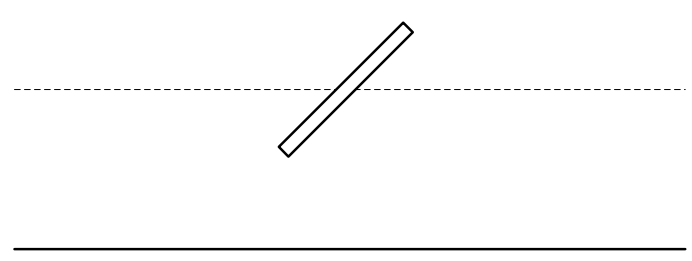

(c) Centered Inclined Series Slot

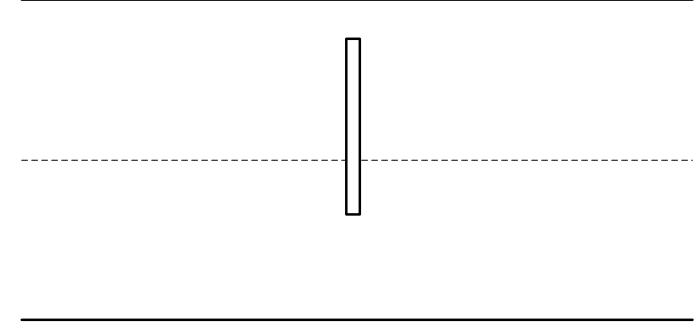

(b) Offset Transverse Series Slot

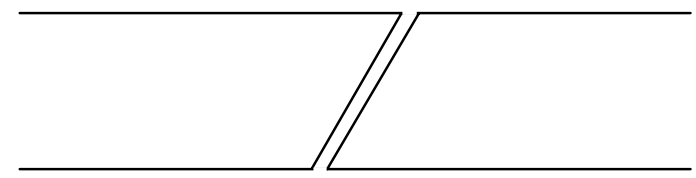

(d) Rotated Shunt Edge Slot

Figure 1 : Linearly Polarized Waveguide Slots

The history of waveguide slot radiator research is long and complex, with several possible dividing points from which to guide a discussion. Here the division will follow slot polarization. The majority of waveguide slot radiator research and applications have focused on linearly polarized slots, so a brief, general historical overview will be presented within a discussion of linearly polarized slots. A more specific discussion of circularly polarized slots will follow. 


\subsection{Linearly Polarized Slots}

The earliest published waveguide slot radiator research was carried out by W.H. Watson in 1945 at University of Saskatchewan. The fruits of this pioneering, highly experimental work have been catalogued in his famous text The Physical Principles of Wave Guide Transmission and Antenna Systems [1-1]. He, like all early waveguide slot researchers, limited his investigation to linear slots cut in either the broad or narrow wall of rectangular waveguide.

The first theoretical analysis was offered by A.F. Stevenson in 1947 at The University of Toronto [1-2]. Stevenson assumed that a slot whose length was equal to half a free space wavelength would be resonant, i.e. have zero reactance/susceptance and, moreover, would have a half-sinusoidal field distribution. He derived expressions for slot resistance and conductance as a function of offset. While his theoretical developments were limited to resonant slots, they had remarkably good agreement with Watson's experimental results and proved a benchmark for all future theoretical waveguide slot developments.

In the late 1950s, A.A. Oliner employed variational methods to the waveguide slot radiator problem [1-3]. In doing so, he overcame the limitations of Stevenson's work and developed approximate expressions to characterize nonresonant slot impedance and admittance. Good agreement was observed with experimental work.

Perhaps the most prolific waveguide slot radiator researcher and author throughout the past 60 years has been R.S. Elliott [1-4 - 1-8]. He was the first person to seriously consider the impact of mutual coupling on the performance of slot radiators in an array environment. Early in his career, he developed standard design approaches for both traveling wave and standing wave array architectures along with the likes of R.C. Hansen [1-9] and A. Dion [1-10]. He later developed iterative techniques to compensate for the influence of mutual coupling on slot design.

In 1974, T.V. Khac presented the first attempt to apply numerical methods to the analysis and design of waveguide slot radiators [1-11]. A specialized Method of Moments (MoM) code was developed to analyze broad wall 
longitudinal and transverse offset slots. Good agreement was shown between experimental, computational, and previously developed analytical results. Many computational studies have been carried out since Khac's work, but little of it had significant historical impact.

B.J. Maxum [1-12] and S.R. Rengarajan [1-13] studied compound slots (i.e., rotated slots offset from the centerline of the broad wall). The former pursued an experimental approach while the latter produced similar computational results.

\subsection{Circularly Polarized Slots}

The most common types of circularly polarized (CP) waveguide slot radiators are depicted in Figure 2. These may be referred to as the T-Slot, X-Slot, and Offset Compound Slot Pair (OCSP).

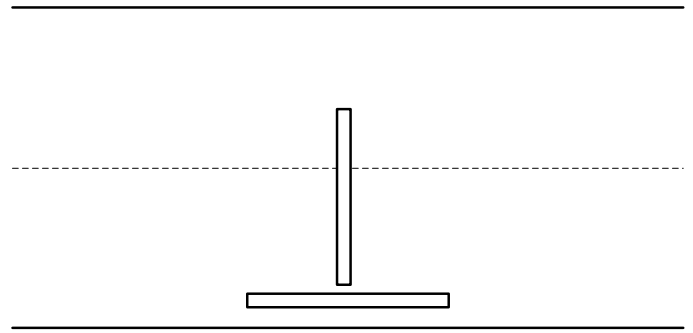

(a) T-Slot

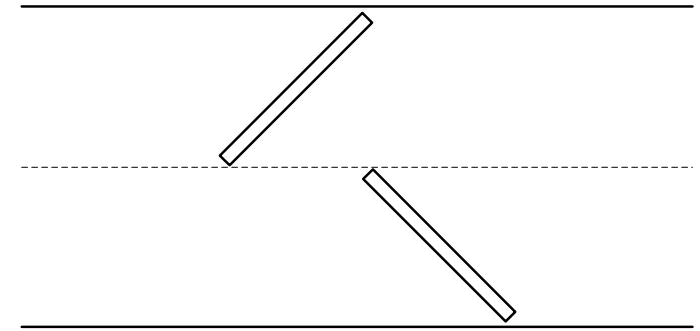

(b) Offset Compound Slot Pair

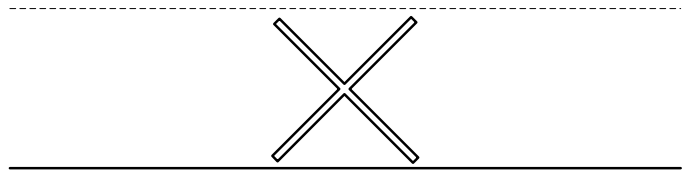

(c) X-Slot

Figure 2 : Circularly Polarized Waveguide Slot Combinations

In contrast to the dense, lengthy, and thorough history of linearly polarized waveguide slot radiator research and development, circularly polarized slot radiators have received only sparse and relatively incomplete attention in the 
literature. In The Physical Principles of Waveguide Transmission and Antenna Systems, Watson describes the possibility of achieving circular polarization from slot pair combinations [1-14] and includes a notional figure for the T-Slot; however no experimental information is presented.

In 1957, A.J. Simmons presented the first experimental results of a circularly polarized waveguide slot [1-15]. Following Watson, and with a surprisingly simple theoretical development, he observed that any slot cut in the broad wall of a waveguide at a properly chosen distance from the center line should radiate circularly polarized energy. After experimenting with several slot shapes, including simple circular holes, he discovered that placing an $\mathrm{X}$-shaped slot, rotated $45^{\circ}$, produced the best axial ratio (AR) and radiated the highest percentage of incident energy.

After Simmons' work, very little theoretical or experimental work was presented on circularly polarized waveguide slot radiators until the 1990s, when the topic was taken up by N. Goto, M. Ando, and J. Hirokawa at the Tokyo Institute of Technology [1-16-1-18]. A unique array topology, employing radial transmission lines and OCSPs was developed for SatCom and mm-wave applications [1-19-1-21].

\subsection{Overview and Goals of Work}

The goal of this work is to propose, develop, and justify a new circularly polarized waveguide slot radiator topology. This new approach will be capable of simultaneous dual-CP operation with co-location of each CP beam, with good axial ratio and return loss performance. Finally, a design methodology employing widely available commercial software tools will be developed to ensure the work is generally accessible.

The remaining chapters are outlined as follows.

Chapter 2 expands on the above historical introduction to waveguide slot radiator research and develops both a context and basis for the main difficulty tackled in this work. The fundamental problems associated with achieving 
simultaneous dual circular polarization with co-located RHCP and LHCP beams will be presented, along with the application spaces where such a system is necessary and waveguide feed systems are advantageous. Finally, an array topology capable of addressing these shortcomings will be presented, including general performance requirements for each component of the array. As will be seen, a deficiency of design information in the literature exists regarding each element in the proposed array topology.

Chapter 3 addresses the primary building blocks of the proposed array topology. Waveguide CP slot radiator options will be compared and a down selected choice justified. Similarly, various approaches to achieving zero interelement phase shift will be compared with a justified down selection. Finally, a design approach will be proposed, highlighting the necessary performance parameters to emphasize during the discussion of the passive phase shifter and slot radiator.

Chapter 4 is a discussion of the slow wave structure passive phase shifter proposed in the array topology studied in this work. A simple, largely derivative theoretical development, including first-order design equations, will be presented for these structures. As will be clearly seen, the first-order textbook design approach insufficiently addresses the performance requirements. In response, a robust design approach employing optimization algorithms and an efficient, widely available piece of commercial software will be developed.

Chapter 5 addresses the $\mathrm{X}$-slot. The basic design equations and theory of operation developed by Watson and Simmons will be presented, along with some simulation results to confirm their findings. Because little design approach information is present in the literature beyond this early work, a parametric study was undertaken and will be presented. The resulting insight provided by the study provides design guidelines for the $\mathrm{X}$-slot within the proposed array topology.

Chapter 6 will cover the complete design methodology for the proposed array topology. A start-to-finish example of a five-element array at X-band will be transparently carried out to further encourage wide accessibility and dissemination of the proposed design approach. The design example will 
conclude with Finite Element Method (FEM) simulation performance for the designed five-element array. FEM results are confirmed via Finite Difference Time Domain (FDTD) simulations.

Chapter 7 will contain concluding remarks and suggestions for future extensions of the present work. 


\section{Chapter 2: The Problems and a Proposed Solution}

\subsection{Dual Circular Polarization}

In certain applications, an antenna capable of simultaneous, dual-circularly polarized (CP) operation is required. Examples of existing systems include DBS, MilStar SatCom, and TDL; while Ka-band military SatCom, HC3, and dualpolarimetric weather radar are over-the-horizon systems which will also require dual CP antennas.

All of the $\mathrm{CP}$ waveguide slot radiators discussed in Chapter 1 are fundamentally capable of dual CP operation. Simply switching sides of the broadwall center line or else changing the direction of an incident feed wave will produce an opposite sense of CP. This phenomenon was observed and reported by Simmons in the earliest $\mathrm{CP}$ waveguide slot paper.

Several modern papers from Tokyo Tech have addressed the problem of achieving dual CP performance from waveguide slot arrays [2-1 - 2-3]. The typical approach taken is depicted in Figure 3. A waveguide feeding mechanism was constructed to provide traveling wave operation via two points at opposite ends of the waveguide feed structure. As demonstrated in the work, the array exhibited simultaneous dual CP operation. However, because the linear arrays were operated under traveling wave conditions, an aperture phase taper existed, producing a beam tilt. The aperture phase taper caused each sense of $\mathrm{CP}$ to produce a beam in opposite directions, also seen in Figure 4. 


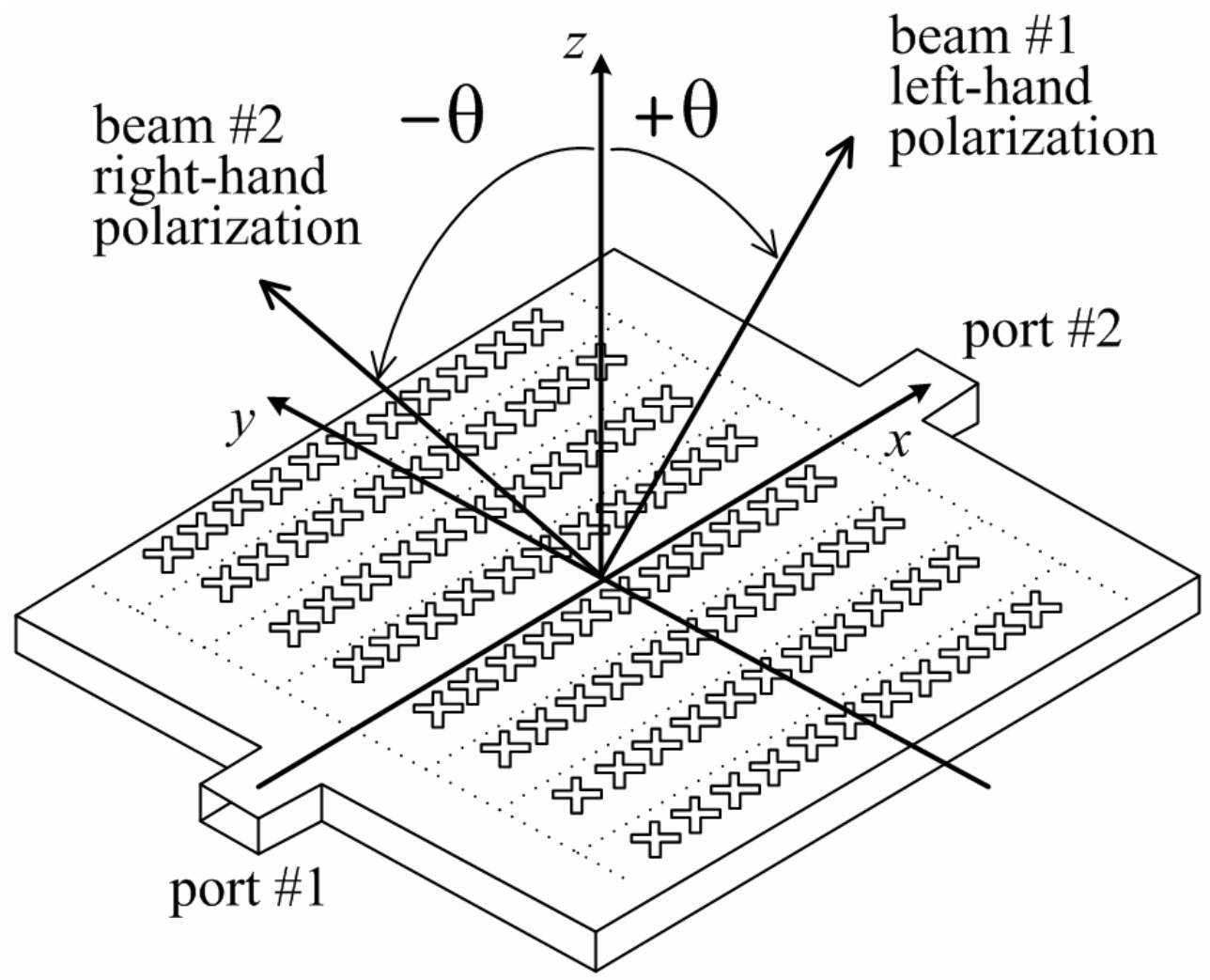

Figure 3 : Typical Dual CP Waveguide Slot Array

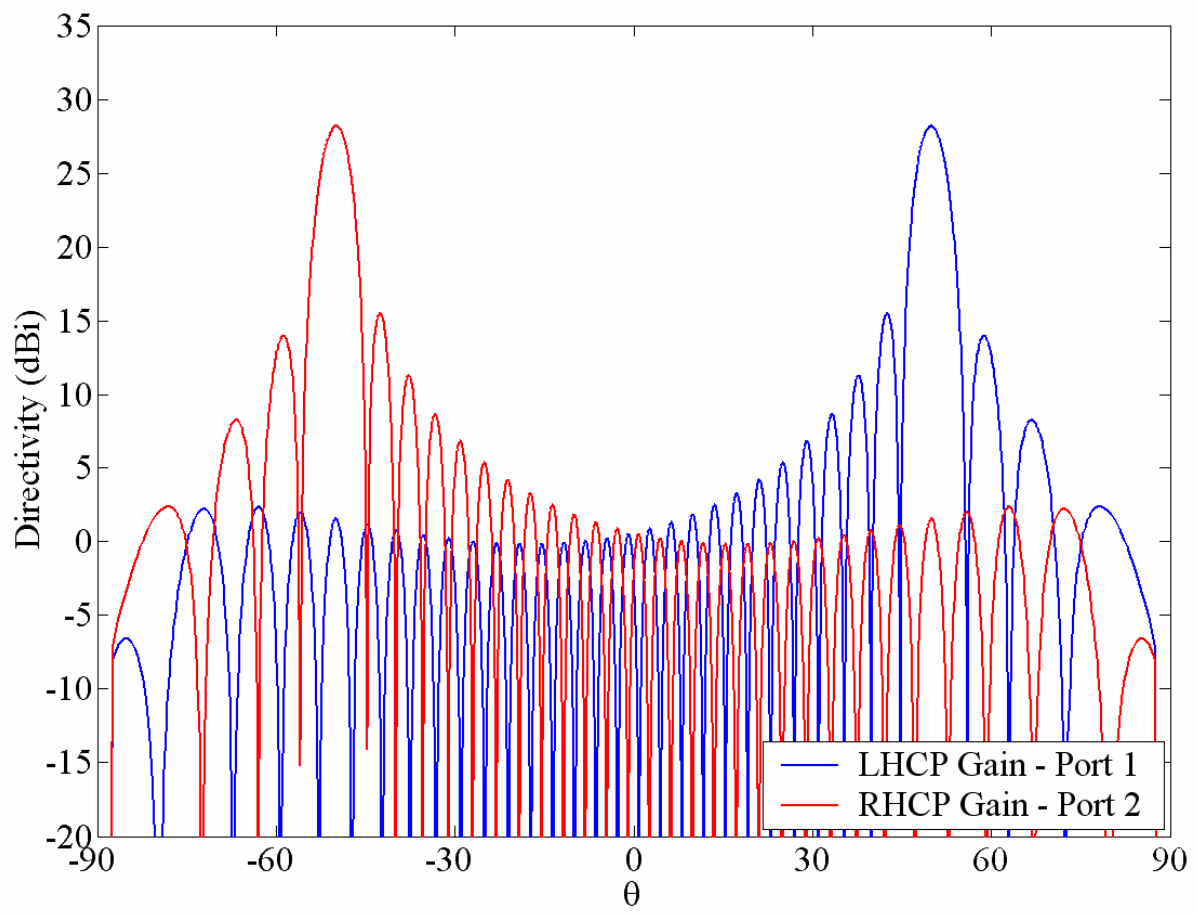

Figure 4 : Typical Dual CP Waveguide Slot Array Gain Pattern 
A series of problems persist relative to achieving dual $\mathrm{CP}$ radiation from waveguide slot arrays. What follows will be a short discussion of these problems and the solutions proposed by this work.

\subsection{The Spatial Beam Divergence Problem}

A feature common to all dual $\mathrm{CP}$ waveguide slot radiators in the literature is the existence of two spatially divergent beams, as seen above. The linear sub arrays must be operated under traveling wave conditions, thus causing an aperture phase taper related to the inter-element spacing (s) and guided wave number $(\boldsymbol{\beta})$.

$$
\Delta \phi=\beta s
$$

The effective aperture phase taper will be of opposite sign when fed from opposite ends of the waveguide. Thus, each sense of CP will produce a beam at an angle $\pm \boldsymbol{\theta}$, around $50^{\circ}$ in the above figure, and determined by the following equation, where $\boldsymbol{\beta}$ and $\mathbf{k}$ represent the guided and free-space wave numbers, respectively:

$$
\sin \theta=\frac{\beta}{k}
$$

Most applications which require dual $\mathrm{CP}$ operation cannot withstand two spatially divergent beams. Consider, for example, a radar system employing dual CP operation - little valuable data could be gleaned from a comparative operation if each beam were pointing at a different target.

In dual CP applications which can tolerate spatially divergent beams, the antennas suffer an intrinsic reduction in aperture efficiency, due to the tilted beam. A common approximation to the aperture efficiency is:

$$
\eta=\frac{A_{e}}{A_{p}} \cos \theta
$$


Here, $\mathbf{A}_{\mathbf{e}}$ is the effective aperture, $\mathbf{A}_{\mathbf{p}}$ is the physical area, $\boldsymbol{\eta}$ is the aperture efficiency, and $\boldsymbol{\theta}$ is the beam angle relative to zenith. As an example, a beam tilt angle of $45^{\circ}$ would reduce the aperture efficiency by around $30 \%$. For applications which demand high aperture efficiencies-a common requirement in ground mobile and land mobile platforms which often restrict antenna real estate - a traveling wave, tilted beam dual CP antenna is a poor choice.

Thus a need exists for the development of a waveguide slot antenna array topology capable of simultaneous dual $\mathrm{CP}$ operation wherein the two $\mathrm{CP}$ beams are located at zenith - i.e. in a direction orthogonal to the plane of the array.

\subsection{The Grating Lobe Problem}

A trivial solution to obtaining broadside radiation with co-located dual CP beams would be to place each slot element a full guide wavelength apart. From (1), it may be seen that the inter-element phase shift would be zero, thus eliminating the aperture phase taper and producing broadside beams. However, the grating lobe condition for a linear array may be stated as

$$
\frac{s}{\lambda}=\frac{1}{\sin \theta_{0}-\sin \theta_{G L}},
$$

where $\boldsymbol{\theta}_{\mathbf{0}}$ is the main beam angle, $\boldsymbol{\theta}_{\mathbf{G L}}$ is the angle of the first grating lobe, $\mathbf{s}$ is the inter-element spacing, and $\lambda$ is the free space wavelength. This expression may be re-written in terms of the minimum inter-element spacing required to suppress grating lobes from visible space:

$$
s \leq \lambda .
$$

Thus, the well-known phenomenon of grating lobes arises if the inter-element spacing is larger than a free space wavelength for the condition of broadside radiation. If the inter-element spacing is set at $\lambda_{\mathrm{g}}$, a cursory glance at the relationship between guided wavelength and free space wavelength provides sufficient insight in this matter. 


$$
\lambda_{g}=\frac{\lambda}{\sqrt{1-\left(\frac{\lambda}{\lambda_{c}}\right)^{2}}}
$$

Within an empty rectangular waveguide, the guided wavelength will always be larger than the free space wavelength. Because the free space wavelength determines the edge of the grating lobe condition, it is impossible to place waveguide slots a full guided wavelength apart - thus achieving broadside radiation-without also having grating lobes. Grating lobes are effectively secondary main beams which have the practical effect of greatly reducing the aperture efficiency.

It should be noted that it is entirely possible to achieve broadside radiation from linearly polarized waveguide slot radiators. A slot field phase reversal may be generated by simply mirroring a longitudinal, broad wall slot across the waveguide center line or alternating the direction of rotation with edge slots in the waveguide narrow wall. In both cases, slots are placed a half guided wavelength apart, thus producing in-phase radiation from elements whose array spacing is less than the grating lobe criteria. Such a phase reversal through simple geometric operations cannot be achieved with any circularly polarized slot.

Thus, it is seen that a need exists to achieve a dual $\mathrm{CP}$ waveguide slot array topology which is capable of co-located $\mathrm{CP}$ beams without grating lobes.

\subsection{Dielectric Loading and the G/T Problem}

A somewhat obvious solution which could achieve broadside radiation from a dual CP waveguide slot array is to dielectrically load the rectangular waveguide cavity. Careful observation of (6) reveals that $\lambda_{\mathrm{g}}$ is dependent upon $\boldsymbol{\lambda}$, that is, the wavelength in an unbound medium with material characteristics equivalent to those found within the waveguide cavity. If the feed waveguide is filled with a dielectric material such that $\lambda_{\mathrm{g}}$ becomes less than $\boldsymbol{\lambda}_{\mathbf{0}}$, the free space wavelength 
and grating lobe condition, broadside radiation may be achieved without grating lobes.

In practice, this approach is rarely employed because several system-level penalties are incurred. First, manufacturing costs and weight are higher for a dielectrically loaded waveguide. Second, the broadwall width must decrease in order to maintain a common single-mode band of operation after the waveguide has been dielectrically loaded. This restricts the range of slot sizes available to achieve CP radiation. Because slot arrays are typically composed of resonant elements, the necessary $\lambda_{0} / 2$ slot length will no longer fit within the dimensions of the broad wall.

Dielectric loading also presents a problem in terms of increasing loss. As will be discussed in Chapter 3, most practical dielectric materials suitable for loading a waveguide will increase the loss of the waveguide more than alternative passive phase shifter architectures.

Increasing feed loss is typically not a design goal, but the precise impact is system specific and difficult to generalize. For example, in a purely terrestrial system (e.g., ground-based, point-to-point data link) the relationship between feed loss and the link margin is purely linear because the antenna noise temperature and feed system noise temperature are equal. By contrast, in a satcom system, where the system noise temperature is in the range of $290 \mathrm{~K}$, while the antenna noise temperature is in the range of $10-50 \mathrm{~K}$, the relationship between feed loss and antenna $\mathrm{G} / \mathrm{T}$ is non-linear. ( $\mathrm{G} / \mathrm{T}$ is the antenna gain divided by the antenna noise temperature and is an important performance metric in determining link margin.) These relationships are brought out in the following equation, where $\mathbf{T}_{\mathbf{A}}$ is the equivalent antenna noise temperature, $\boldsymbol{\eta}_{\mathbf{r a d}}$ is the antenna radiation efficiency, $\mathbf{T}_{\mathbf{B}}$ is the background noise temperature (10-30K for daytime reception of signals above the horizon), $\mathbf{T}_{\mathbf{P}}$ is the physical temperature of the antenna $(290 \mathrm{~K})$, and $\mathbf{L}$ is the loss in the feed network:

$$
T_{\text {sys }}=\eta_{\text {rad }} T_{B}+\left(1-\eta_{\text {rad }}\right) T_{P}+(L-1) T_{P}
$$


If the background noise temperature and the antenna physical temperature are identical, the net amount of noise present in the system at the output of the antenna will not increase with feed loss. If, however, they are unequal, the net effect of feed loss will be to both decrease the gain of the antenna and increase the effective noise temperature at its output. Thus, while increased feed loss will always decrease performance, the level to which it does so is not easily generalized and is system specific.

It is seen that a need exists to develop a dual CP waveguide slot array topology which is capable of co-located CP beams without grating lobes through some avenue other than dielectric loading. This is generally true from a manufacturing stand point and finds special relevance in satcom systems where feed loss non-linearly reduces system performance.

\subsection{The Design Problem}

A final problem associated with dual CP waveguide slot arrays is the void of design information available in the open literature. X-slots are by far the most common CP slot found in publication, yet the only design equation or design information available is found in the original paper by Simmons in 1957! An occasional reference has been made to experimental studies having been carried out to characterize the X-slot design process [2-4], but no useful results have been made openly available.

A clear need exists for a generally available, parametric study which would reveal the salient design parameters for X-slots above and beyond the relationship provided by Simmons. A goal of this work is to undertake such a study and determine a set of design rules for X-slots in a dual CP waveguide slot array. Chapter 5 covers this topic.

A second design difficulty arises when the slow wave structure passive phase shifter is considered. Well-defined analytical methods have been successfully employed and are widely available to determine the slow wave characteristics of infinitely periodic structures within waveguide (capacitive and 
inductive irises, corrugations, etc.) [2-5 - 2-7]. However, a more general methodology to design well-matched slow wave structures of only a few elements has not been presented.

Thus, a clear need also exists for a slow wave structure passive phase shifter design procedure. A goal of this work is to provide such a design procedure based on the strong analytical foundations that were laid over 50 years ago and extend them to a modern design approach of optimization via efficient computational methods. Chapter 4 covers this topic.

Finally, any design methodology that would be considered widely available in a modern context must be amenable to the methods and tools which are currently widely employed. In the 1950s, when Watson, Stevenson, and Oliner carried out their pioneering works, the then-modern design tools included slide rules and design curves. In the late 1970s, when Khac and Elliott began applying numerical methods-MoM, in particular - to the problem of waveguide slots, a new tool came into limited usage - the digital computer. Over the years since the pioneering efforts, much of the new tools developed have followed this trend - that is, specialized code packages were developed to analyze a particular waveguide slot radiator design problem. Unfortunately, these techniques were not widely available as each designer had to write a unique piece of code (MoM, typically) to tackle each problem.

In the modern era, the accuracy and price of both commercially available generic numerical E\&M solvers employing standard methods (FEM, MoM, FDTD, etc.) and the necessary computing resources to utilize them, has revolutionized the face of professional electromagnetics. Today, the widely available tools are HFSS [2-8], CST [2-9], FEKO [2-10], and the like, and allow users - in academia and industry alike - a great deal of design flexibility and accuracy without the prohibitive cost seen in the early days of Khac and Elliott.

A primary philosophical goal of this work is to develop a design methodology which uses these commercially available tools to ensure the results of this work will be as widely available as the software tools themselves. Additionally, more than one tool will be employed because, despite the accuracy 
of modern numerical methods, each approach has its strengths and weaknesses and an efficient design approach must utilize the numerical approaches that are optimally suited for the each task.

\subsection{A Solution Approach}

A proposed solution to the problem of achieving a dual CP waveguide slot array topology featuring co-located CP beams with grating-lobe-free, broadside radiation, and good return loss without the use of performance limiting dielectrics is depicted in Figure 5. The decision to choose X-slot radiators is discussed in the next chapter. Broadside radiation without grating lobes is achieved via slow wave structure passive phase shifters. They permit inter-element spacings less than a free space wavelength while enabling zero inter-element phase shift. The architectural details, array topology, and a design approach will be further discussed in the next chapter.

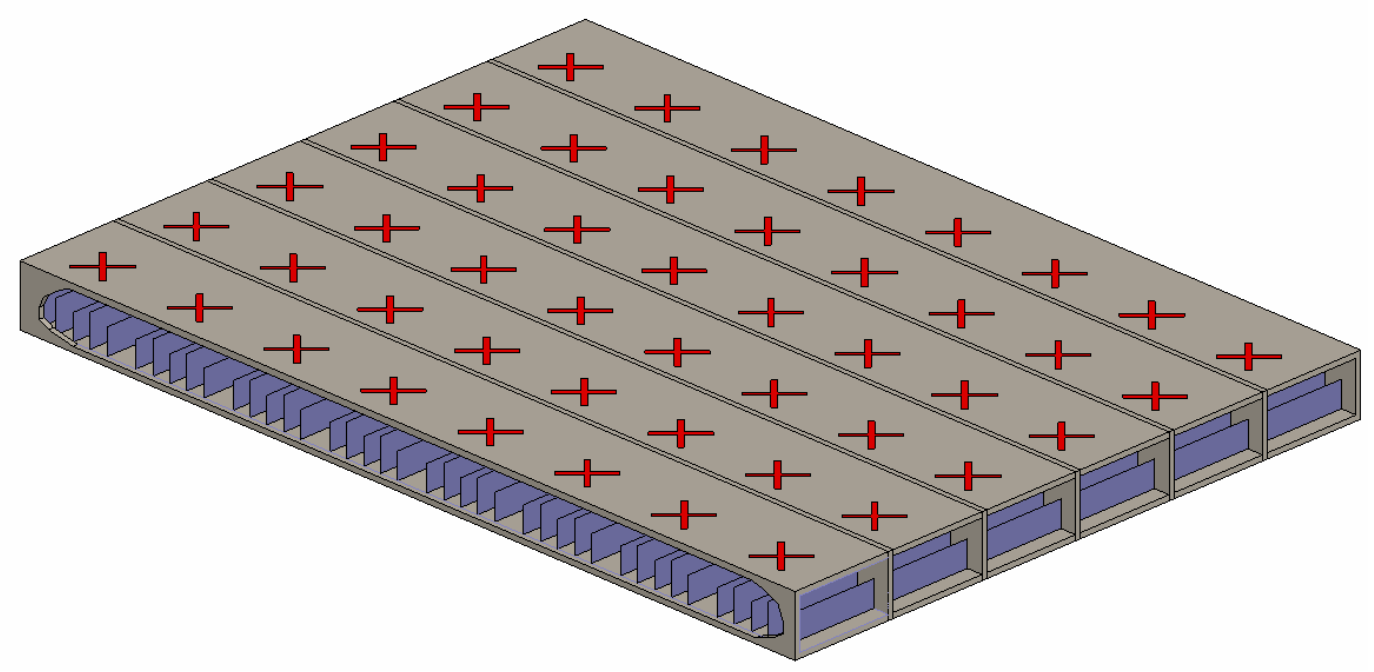

Figure 5 : Proposed Dual CP Array Topology

Architecturally similar approaches have been suggested in the past [2-11 $\&$ 2-12] and are being researched at present [2-13] for $\mathrm{CP}$ waveguide slot radiators with broadside radiation. However, a philosophical difference exists between these approaches and that proposed here. In these papers, continuously 
distributed corrugated waveguide structures are employed. This causes a distributed shift in the effective guide wavelength and necessitates a coupled design procedure, whereby the slot radiators and slow wave structures must be designed simultaneously. By restricting the length of the phase shifter unit cell, coupling between the slot radiators and phase shifters may be treated from simple transmission line theory, allowing an independent, simplified design procedure for each component. The design procedure is further simplified by assuming each element in the cascaded network has a low return loss. A final advantage of the passive phase shifters suggested in this work relative to uniformly distributed corrugations is the reduction in fabrication time.

A similar array layout of slow wave structure passive phase shifters has been suggested for traveling-wave arrays of linearly polarized, transverse slots [214]. This brief work, in failing to present a design approach and ignoring manufacturing issues, does little more than lend credence to the approach of employing fairly simple slow wave structure passive phase shifting elements to achieve broadside radiation without grating lobes in a traveling wave slot radiator array where slot field phase reversal is not possible.

The primary goals of this work are to confirm the performance characteristics of the proposed dual CP array topology and develop a widely available design procedure for a linear subarray. Numerical simulation will be the primary tool to confirm device operation. The majority of the work will employ the FEM approach with FDTD used to substantiate the FEM results. 


\section{Chapter 3: Array Topology and Design Approach}

\subsection{The Array Topology}

The proposed array topology includes an X-slot and slow wave structure passive phase shifters. The radiating element has a well-known capacity for dual CP operation when fed from opposite ends of a linear subarray. The slow wave structure passive phase shifter provides the required phase shift to permit broadside radiation. A series of images depicting the linear subarray and each of the linear subarray components are found in Figure 6. What follows is a discussion of the radiator and phase shifter candidates, their relative merits, and the impetus for choosing this array layout.

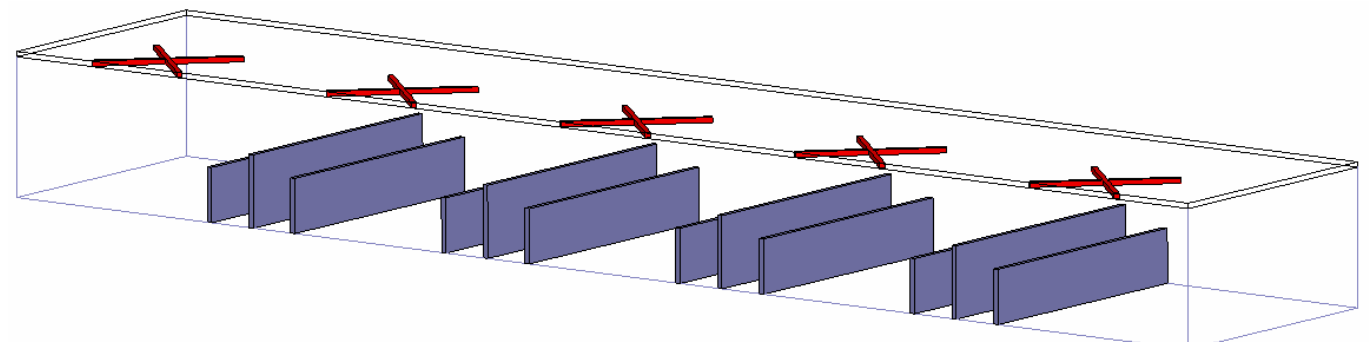

(a) Isometric View

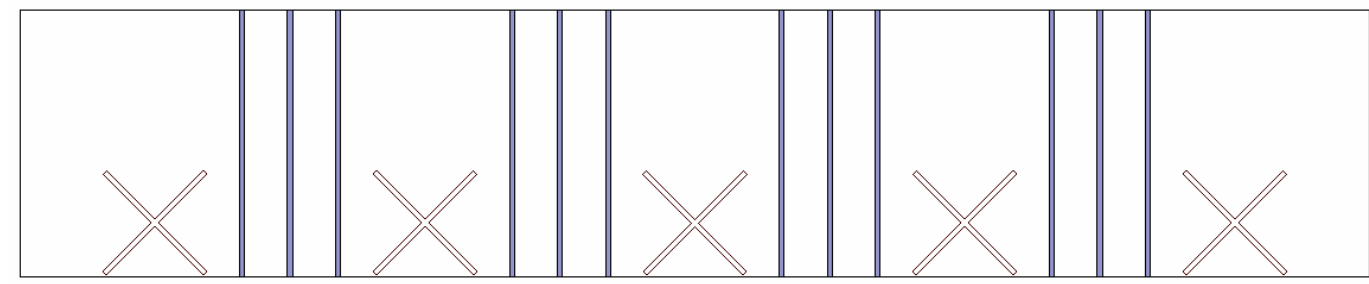

(b) Top View

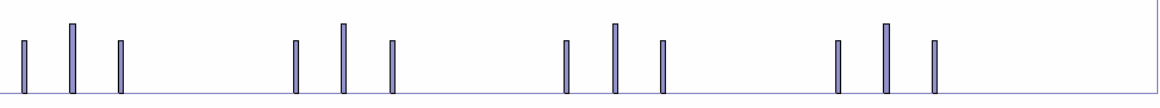

(c) Side View

Figure 6 : Proposed Linear Subarray 


\subsubsection{The Radiating Elements}

As discussed above, three historical CP slot radiators exist - the T-slot, X-slot, and Offset Compound Slot Pair (OCSP), seen in Figure 2, in the broad wall of a rectangular waveguide.

The means by which each slot configuration achieves $\mathrm{CP}$ radiation is virtually identical. It is a fairly simple exercise to derive the fields within a rectangular waveguide for any TE or TM mode of operation, starting from Maxwell's equations and prescribed boundary conditions. The H-field expressions for the most common mode, $\mathrm{TE}_{10}$, are stated below with Figure 7 included for reference. (In the figure, the width of the waveguide broad wall is referenced by $\mathbf{a}$.)

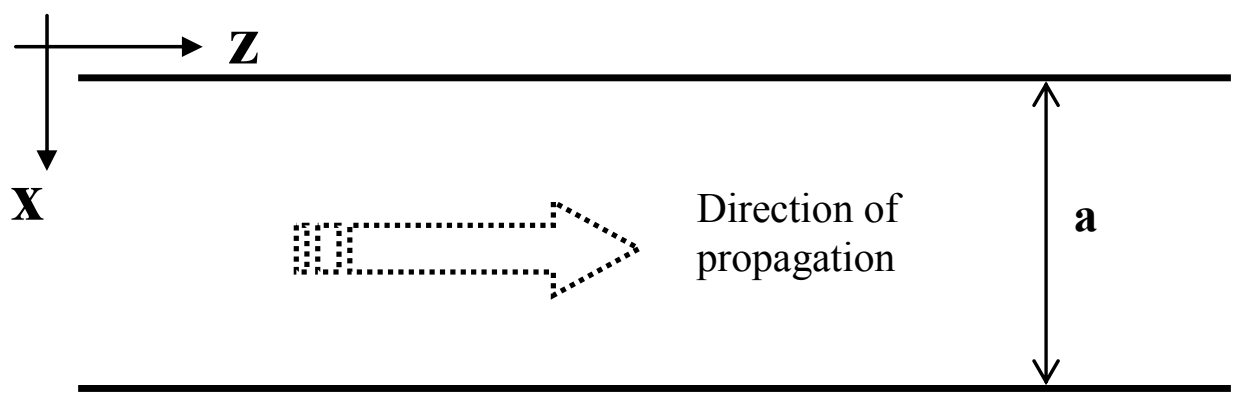

Figure 7 : Rectangular Waveguide Broad Wall

$$
\begin{aligned}
& H_{x}=H_{0} \cos \left(\frac{\pi x}{a}\right) e^{-j \beta z} \\
& H_{z}=j \frac{\beta a}{\pi} H_{0} \sin \left(\frac{\pi x}{a}\right) e^{-j \beta z}
\end{aligned}
$$

The $\mathrm{H}$-fields are important in this discussion because they may be considered the driving source components of the magnetic current formed in the 
waveguide slot. The magnetic slot currents generate aperture E-fields which radiate. An alternative method to visualize the mechanism by which waveguide slots radiate is through the disruption of surface currents in the broad wall of the waveguide. If a slot discontinuity is created within the conducting sheet that forms the waveguide wall, electrons are forced to flow around the slot, thus producing magnetic currents along the slot length and displacement currents across the slot width. These slot aperture fields radiate.

Two important observations can be made from (8) and (9). First, the orthogonal magnetic field components are in quadrature - i.e., their phases are separated by $90^{\circ}$. This is a necessary element in circular polarization. Second, the magnitudes are not equal through the transverse dimension. The longitudinal field has an odd distribution whose peak value is a function of frequency; while the transverse field maintains an even distribution whose peak magnitude is invariant with frequency. Further visualization of these fields is provided in Figure 8 and Figure 9.

Fundamentally, each of the CP slot configurations above is coupling to these two fields to produce circularly polarized radiation. Each of the slot configurations utilize the fields in slightly different fashions, but they are all capable of radiating two orthogonal fields of equal magnitude in phase quadrature - the three necessary conditions for circularly polarized radiation.

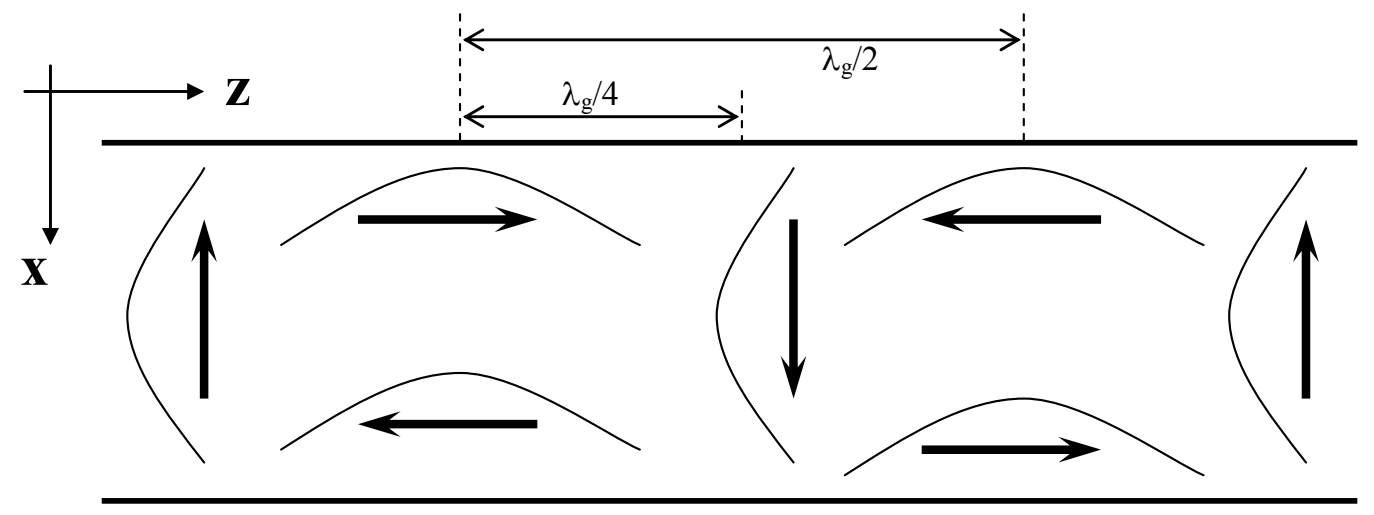

Figure $8: \mathrm{TE}_{10}$ Notional Magnetic Field Distribution 


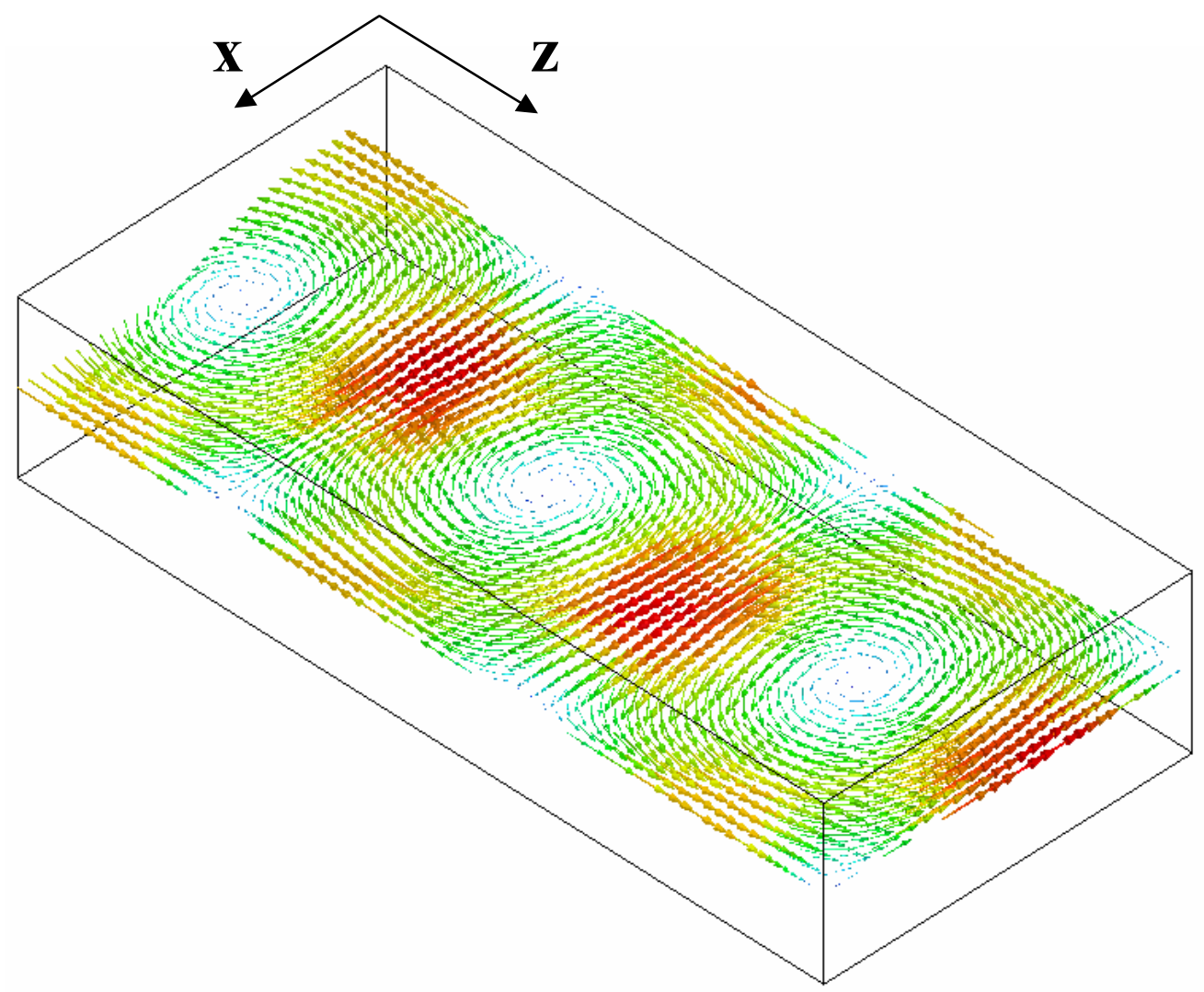

Figure 9 : TE $_{10}$ Magnetic Field Vector Plot

\subsubsection{T-Slot}

Of the three slot combinations, the T-slot is perhaps the simplest to comprehend and is, not surprisingly, the earliest reported CP slot in the literature [1-14]. The T-slot is composed of two classic linearly polarized slot radiators - the offset longitudinal shunt slot and the transverse series slot. Because each slot has a strong linear polarization, its source field is independent of the partnering slot that is, the two aperture fields do not mutually couple. The longitudinal slot couples strongly to the longitudinal magnetic field while the transverse slot couples strongly to the transverse magnetic field. The source fields are in quadrature and the apertures are orthogonal, thus supplying two of the conditions for $\mathrm{CP}$ radiation. The third condition of equal magnitude in the orthogonal field components is somewhat more challenging to achieve with T-slots. 
The slot aperture field magnitude is controlled by the amount of offset in both of these slots. However, the range of achievable resistance values for the transverse slot in this pair is not equal to that of the longitudinal slot. Thus, the range of coupling values that could be employed in a CP array design is limited by the transverse slot's range. The relatively limited range of values that can be achieved has been one of the reasons why it is so rarely employed in waveguide slot arrays [3-1].

To determine which of the $\mathrm{CP}$ slot topologies is most appropriate for the array at the center of this work, simple unit cell simulations were carried out. Each type of CP slot was fed from an identical rectangular waveguide and permitted to radiate into a half space, bound by an infinite ground plane (surrounding the slot) and a Perfectly Matched Layer (PML) radiation condition. All conductors were considered lossless and all non-conductor regions were modeled as lossless vacuum. Dual CP radiation patterns and S-parameter data were evaluated to determine the AR, coupling values, and return loss performance metrics. All CP slot simulations were carried out with Ansoft HFSS.

An image of the two-port T-slot model generated for simulation is seen in Figure 10. When the T-slot pair was fed from Port 1, the radiation pattern in Figure 11 was calculated. The radiation pattern calculated when port 2 was excited is seen in Figure 12. Ports 1 and 2 were not labeled in the unit cell image, but may be understood as two parallel waveguide sources at opposite ends of the unit cell. The same convention will be applied to the discussion of the OCSP and X-slot, below. 


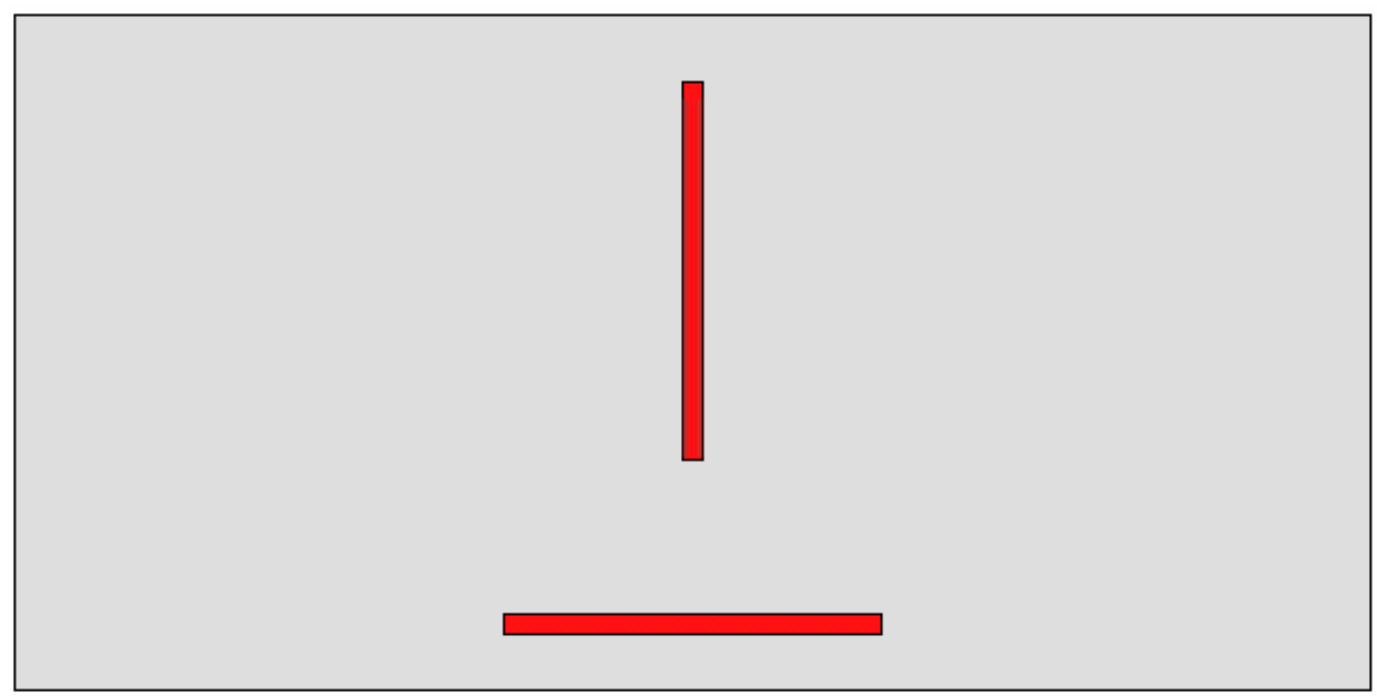

Figure 10 : Simulated T-Slot

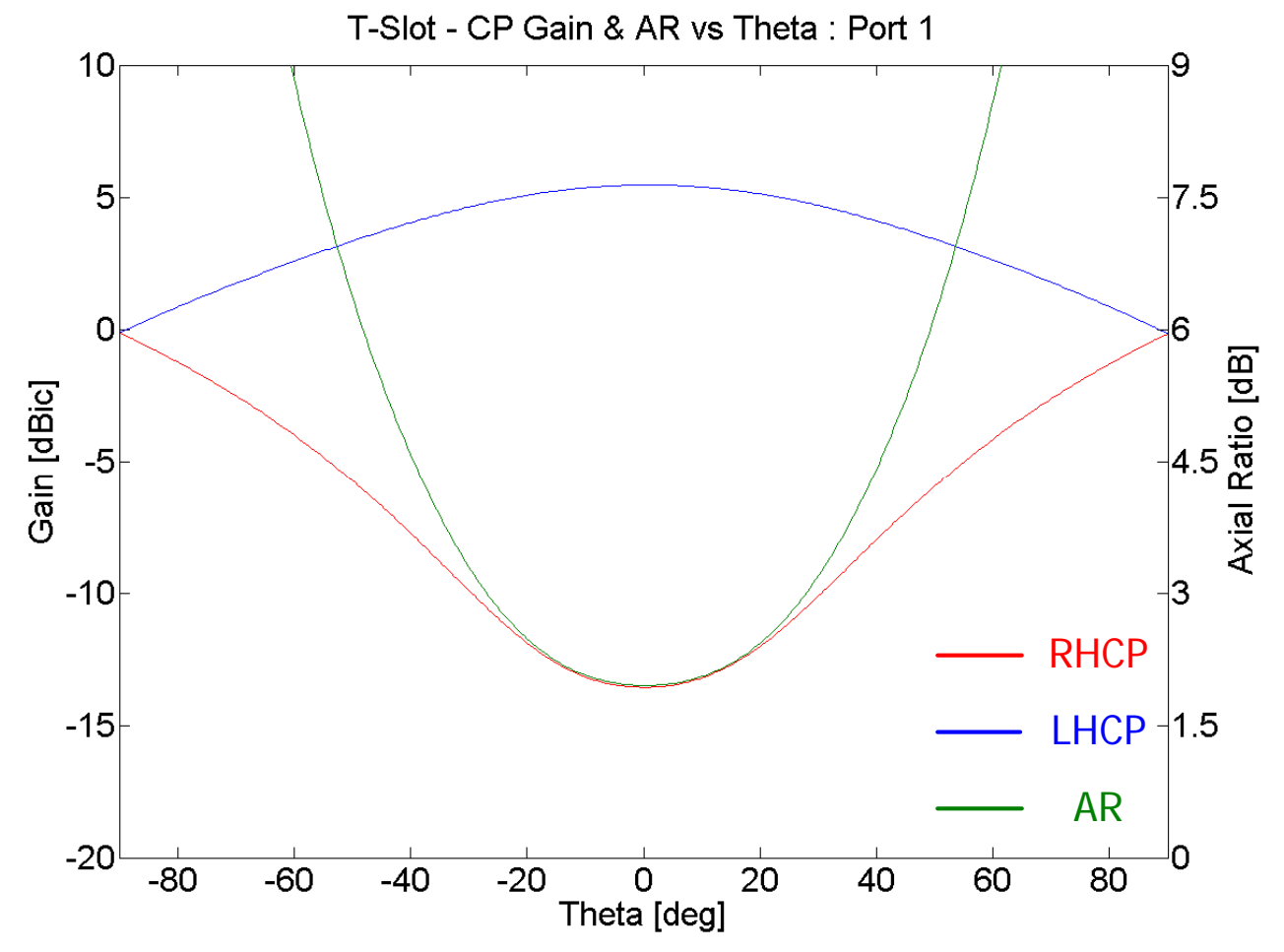

Figure 11 : T-Slot LHCP Pattern 


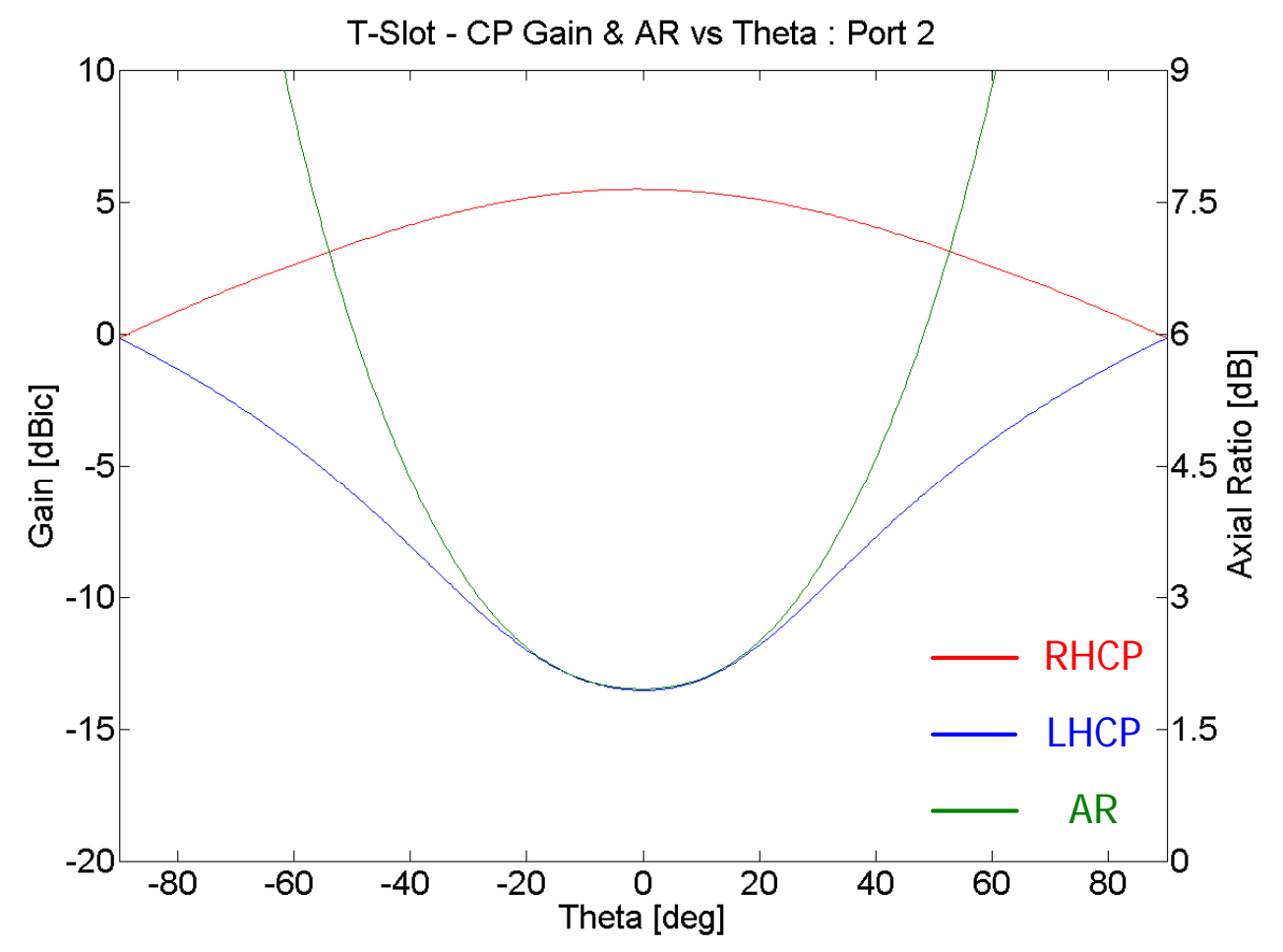

Figure 12 : T-Slot RHCP Pattern

As anticipated, the T-slot provided good $\mathrm{CP}$ radiation with a switch of $\mathrm{CP}$ sense generated via alternating the source field direction of propagation. To further investigate the radiation properties of the T-slot, plots were generated for the entire half space into which the T-slot radiated. In Figure 13 and Figure 14, the RHCP and LHCP gain patterns were calculated when only one port was excited. The terms, co-polarized $(\mathrm{CoPol})$ and cross-polarized (XPol) are employed to indicate the gain associated with the dominant hand of CP radiation and its opposite. From above, LHCP is the CoPol response for Port 1, while it is XPol for Port 2.

The half-space radiation plots are gain values calculated on the surface of a hemisphere and projected onto a circle. The center point of the plot represents zenith $(\theta=0, \varphi=0)$. Movement radially outward is equivalent to an increase in $\theta$, i.e. movement toward the horizon. Movement circumferentially is equivalent to a change in $\varphi$. 
As expected the co-polarized gain pattern was fairly uniform over the hemisphere. The two gain anomalies present in the upper right and upper left corners of the CoPol plot can be understood in terms of an array factor. The phase centers of each slot comprising the T-slot pair are separated slightly. The physical separation and quadrature phase relationship combine to produce a slight end-fire radiation pattern. That is, the main beam of the array factor exists away from zenith, toward the bottom of the plot and nulls appear near the top of the plot, also due to the array factor. Hence, the co-polarized gain pattern is shifted slightly downward and nulls appear near the horizon opposite the direction of the peak shift.
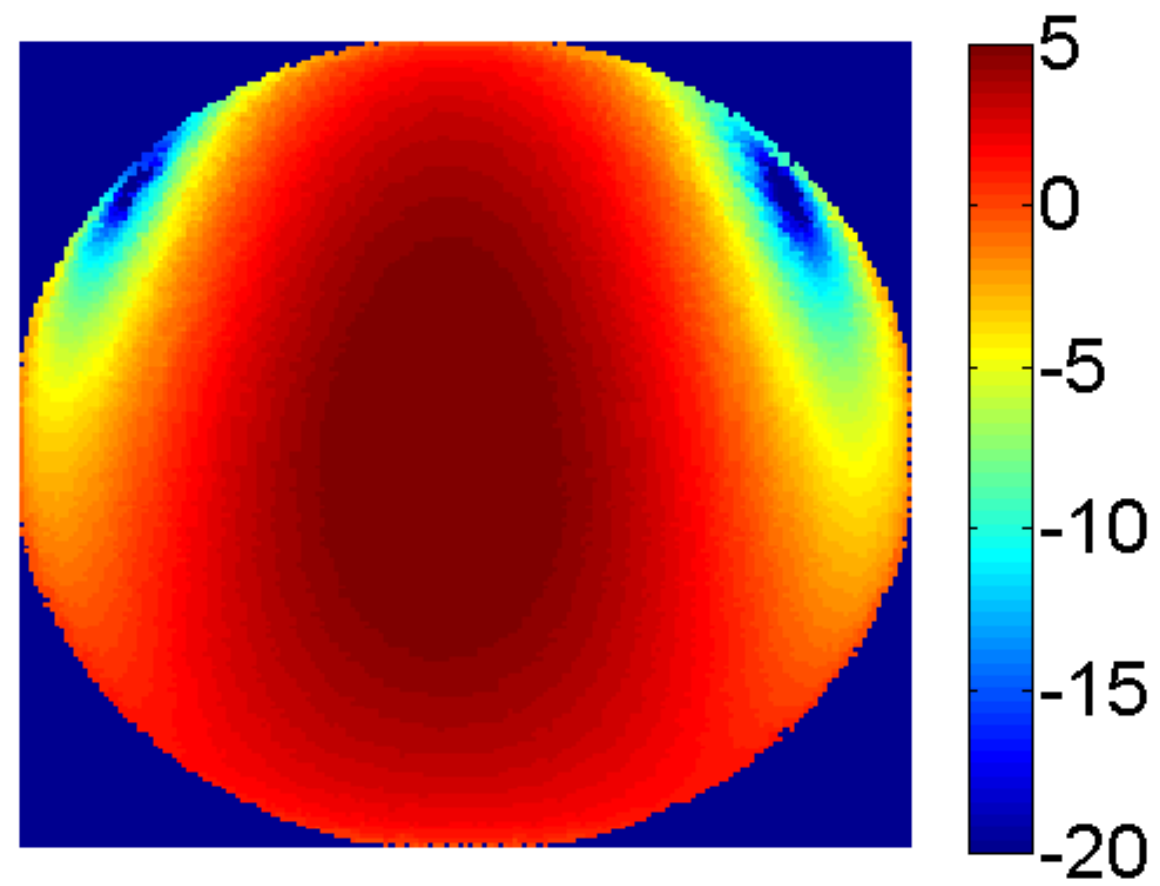

Figure 13 : T-Slot Half Space CoPol Gain (dB) 


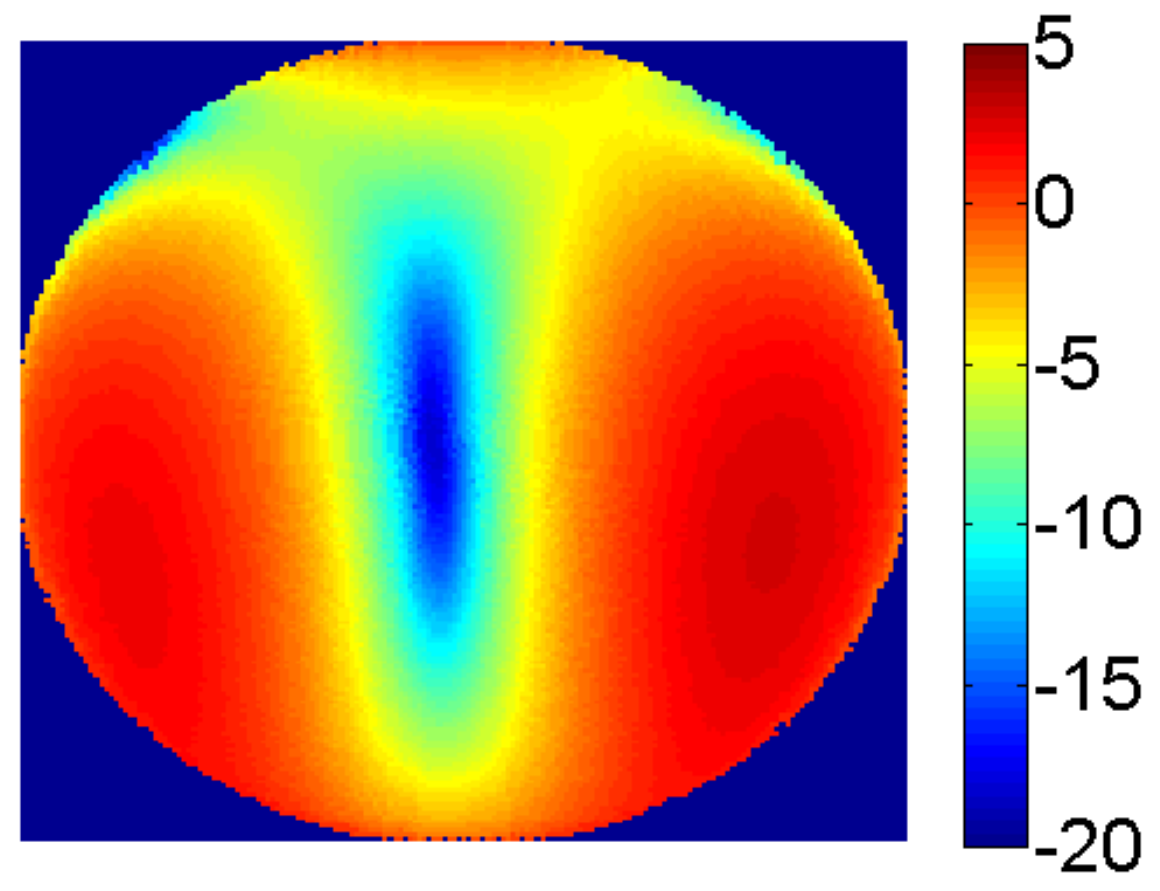

Figure 14 : T-Slot Half Space XPol Gain (dB)

Finally, the T-slot s-parameter performance data is presented in Figure 15. The $S_{21}$ and $S_{11}$ magnitudes exhibit the range of values necessary for a traveling wave slot array design. That is, a wide range of $S_{21}$ values were observed over which the return loss was low enough to ignore reflections. It should be noted that the $S_{21}$ phase was calculated by removing the effects of the waveguide transmission line separating the port in the simulation model. The phase plane of each port was de-embedded to the center of the T-slot. The goal of this shift was to calculate the forward scattered phase perturbation the T-slot presented to the waveguide source field. The relevance of obtaining the $S_{21}$ phase data in this format will be made clear below in a discussion of the design approach. 


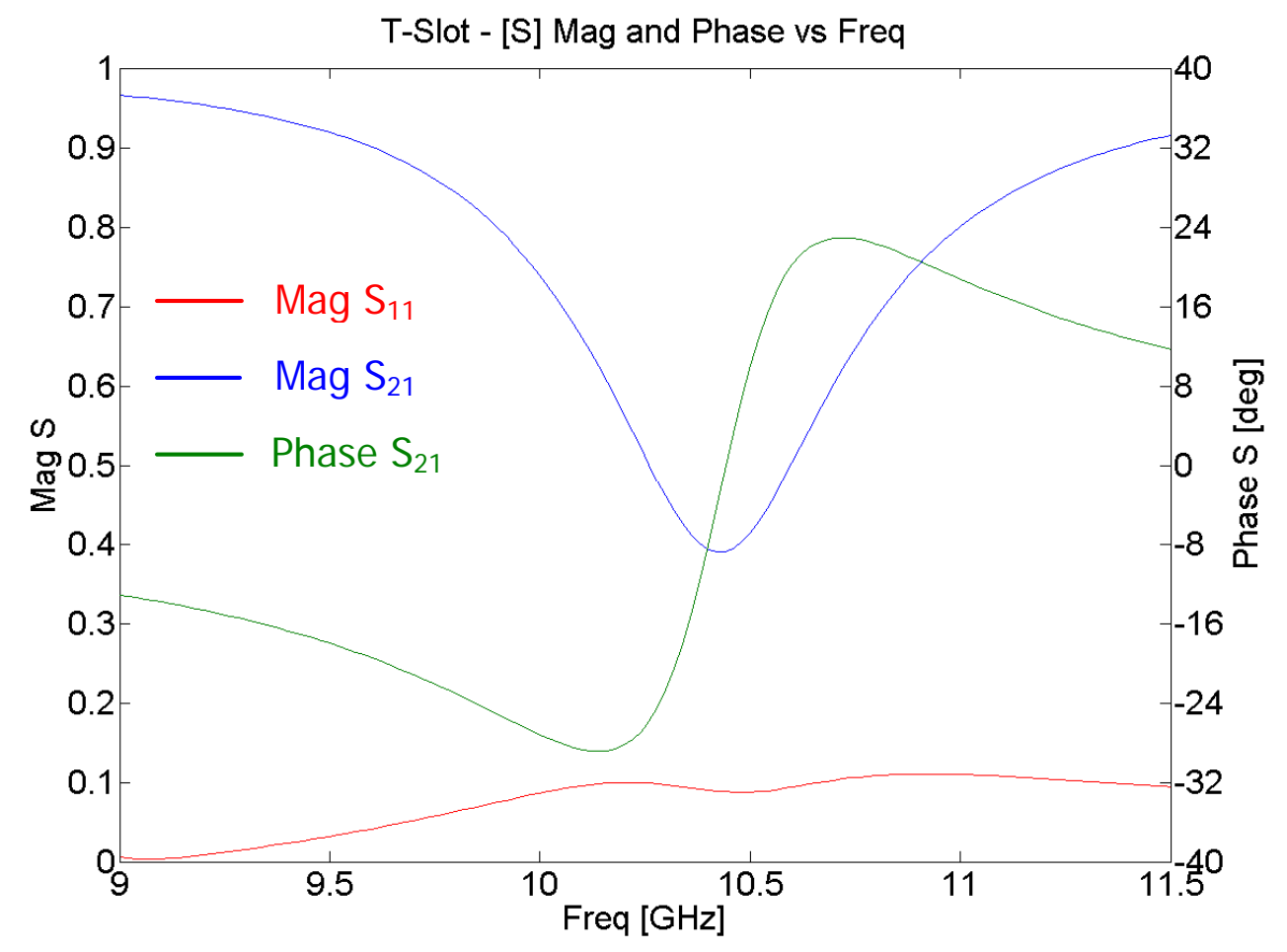

Figure 15 : T-Slot S-Parameter Performance

\subsubsection{Offset Compound Slot Pair}

A single, rotated slot offset from the center line of the broad wall was first studied by B.J. Maxum in 1960 [1-12]. It was further studied by S.A. Rengarajan in the early 1990s [1-13], who applied the "compound" label. The slot is so termed because it cannot be represented by either a simple shunt or series circuit. The slot interacts with both the transverse and longitudinal source fields, thereby compounding the problem and making an equivalent circuit analysis unreasonable.

The combination of compound slots to produce $\mathrm{CP}$ radiation from a rectangular waveguide feed line is a relatively new development, being first considered by Montisci, Mazzarella, and Musa in 2003 [3-2 and 3-3]. This slot configuration generates $\mathrm{CP}$ radiation by rotating an offset slot to $45^{\circ}$ from the center line of the waveguide and mirroring the slot about this line. The second, orthogonal slot is then translated $\lambda_{\mathrm{g}} / 4$ in the dimension of propagation to ensure 
quadrature fields. $\mathrm{CP}$ radiation is possible, because each slot is identical, orthogonal, and at an electrical separation of $90^{\circ}$.

As with the T-slot, a simple simulation was carried out with a characteristic OCSP in HFSS. An image of the model employed is seen in Figure 16.

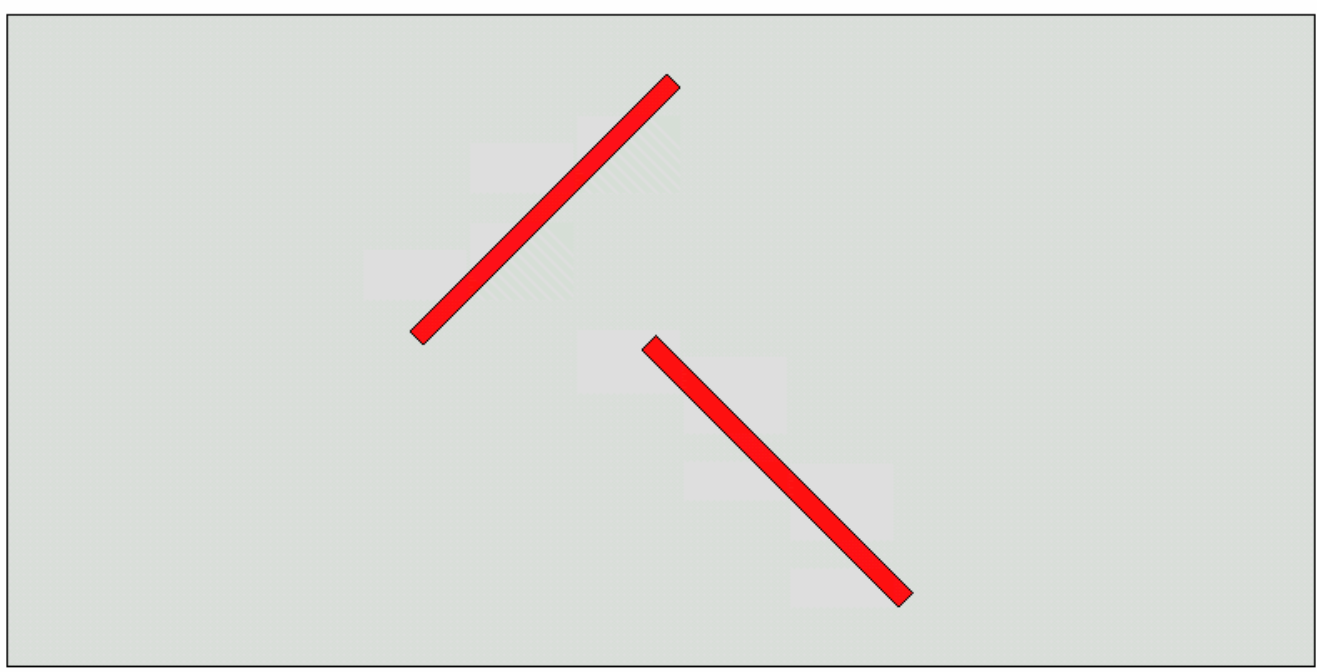

Figure 16 : Simulated Offset Compound Slot Pair

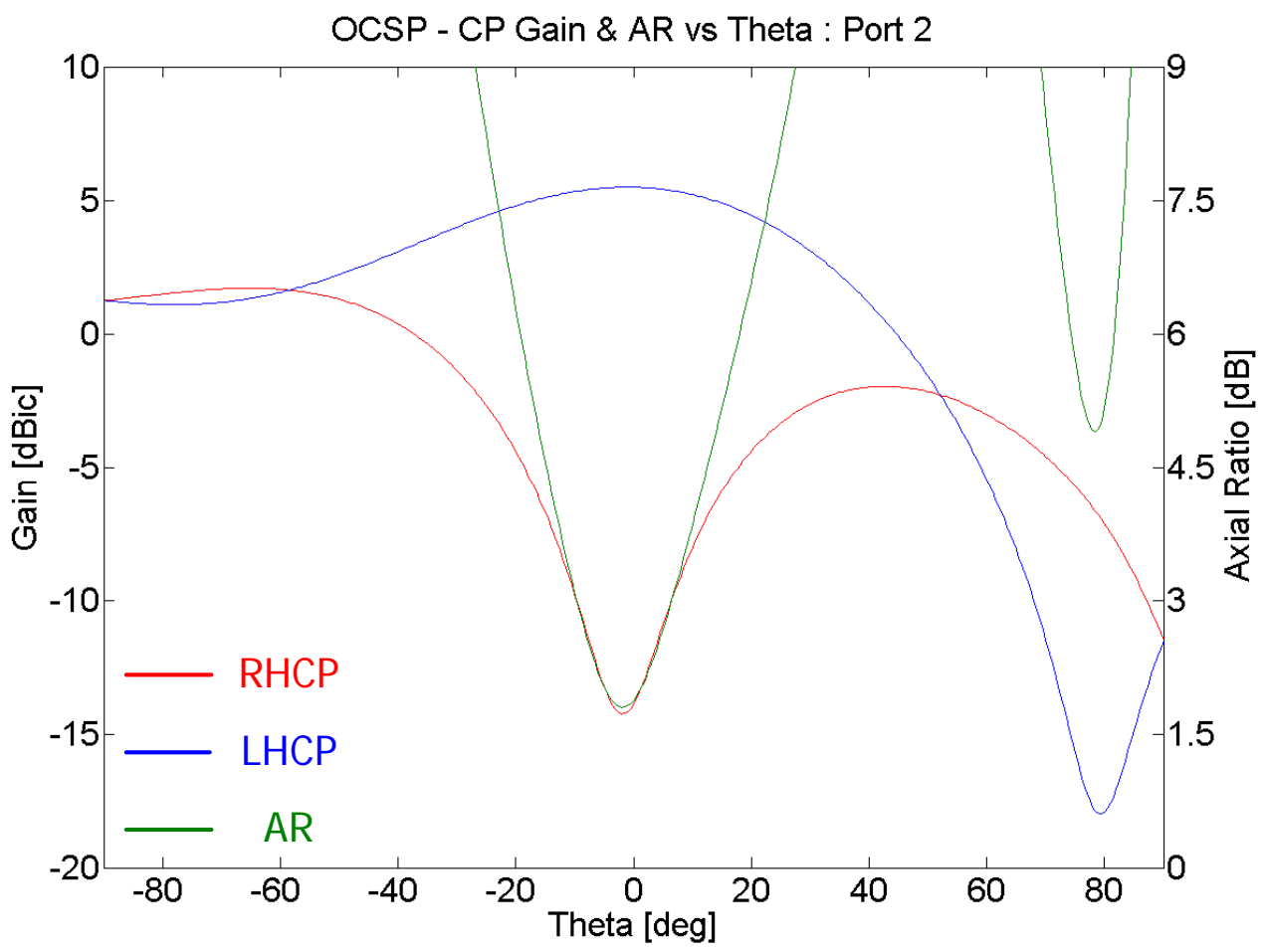

Figure 17 : OCSP LHCP Pattern 


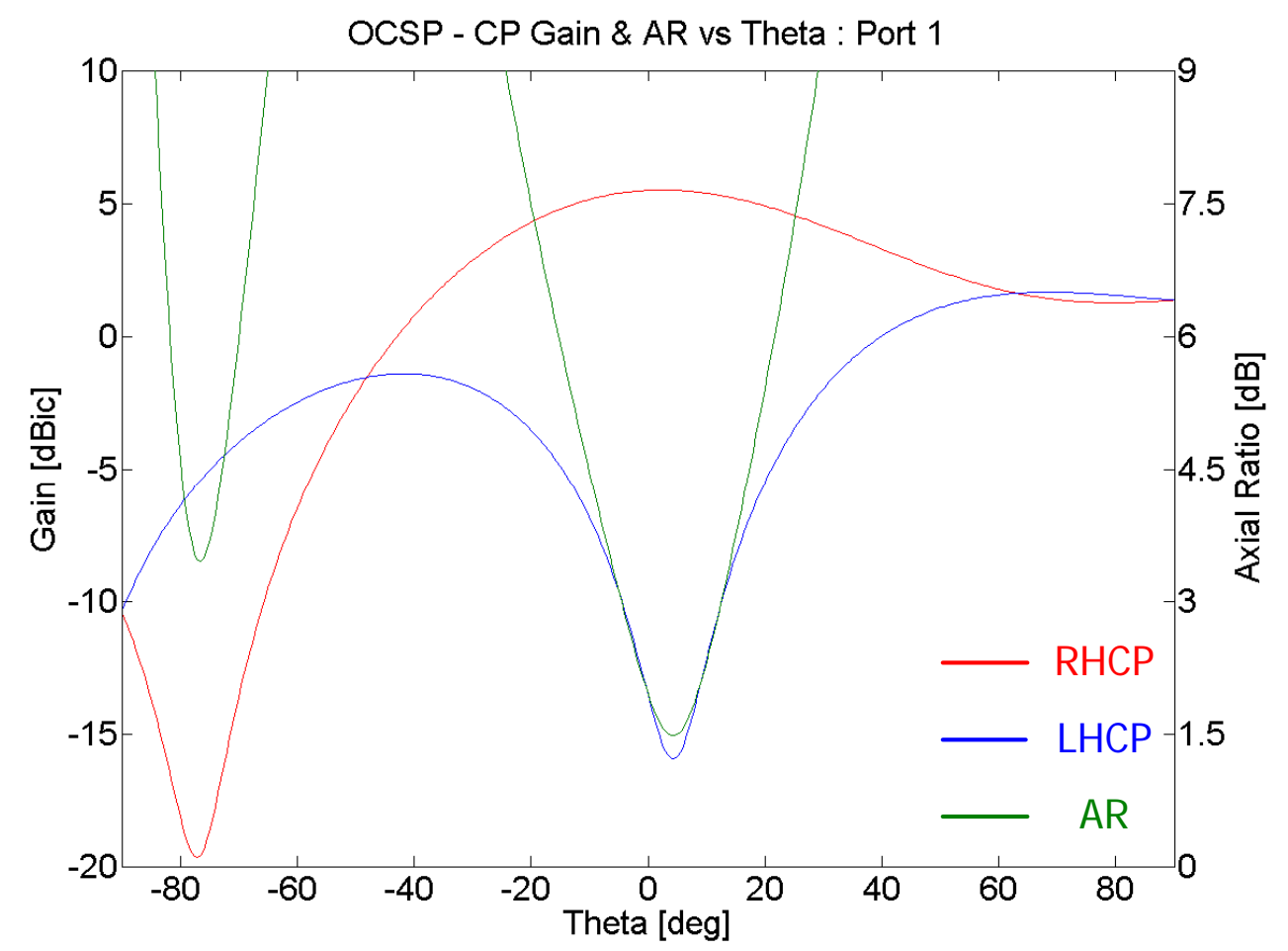

Figure 18 : OCSP RHCP Pattern

The OCSP radiation patterns (Figure 17 and Figure 18) exhibited good AR at zenith, as well as good symmetry between the solutions from each port excitation. Each pattern, however, included a curious asymmetry beyond $20^{\circ}$ to either side of zenith. Another study, not presented here for the sake of brevity, demonstrated that this asymmetry can be attributed to the separation of each slot's phase center. Because the two slots are separated by $\lambda_{\mathrm{g}} / 4$ and are driven in phase quadrature, their combined array factor produces a beam tilted away from zenith, along the axis formed between the slot phase centers. As with the T-slot, examining the radiation patterns in the entire half space provides additional information about this phenomenon.

The lower left corner of both Figure 19 and Figure 20 corresponds to the direction of the main beam of the array factor. The asymmetry seen in the previous plots is an artifact of the original radiation pattern being calculated on an inter-cardinal plane relative to the major axis of the OCSP array factor. 


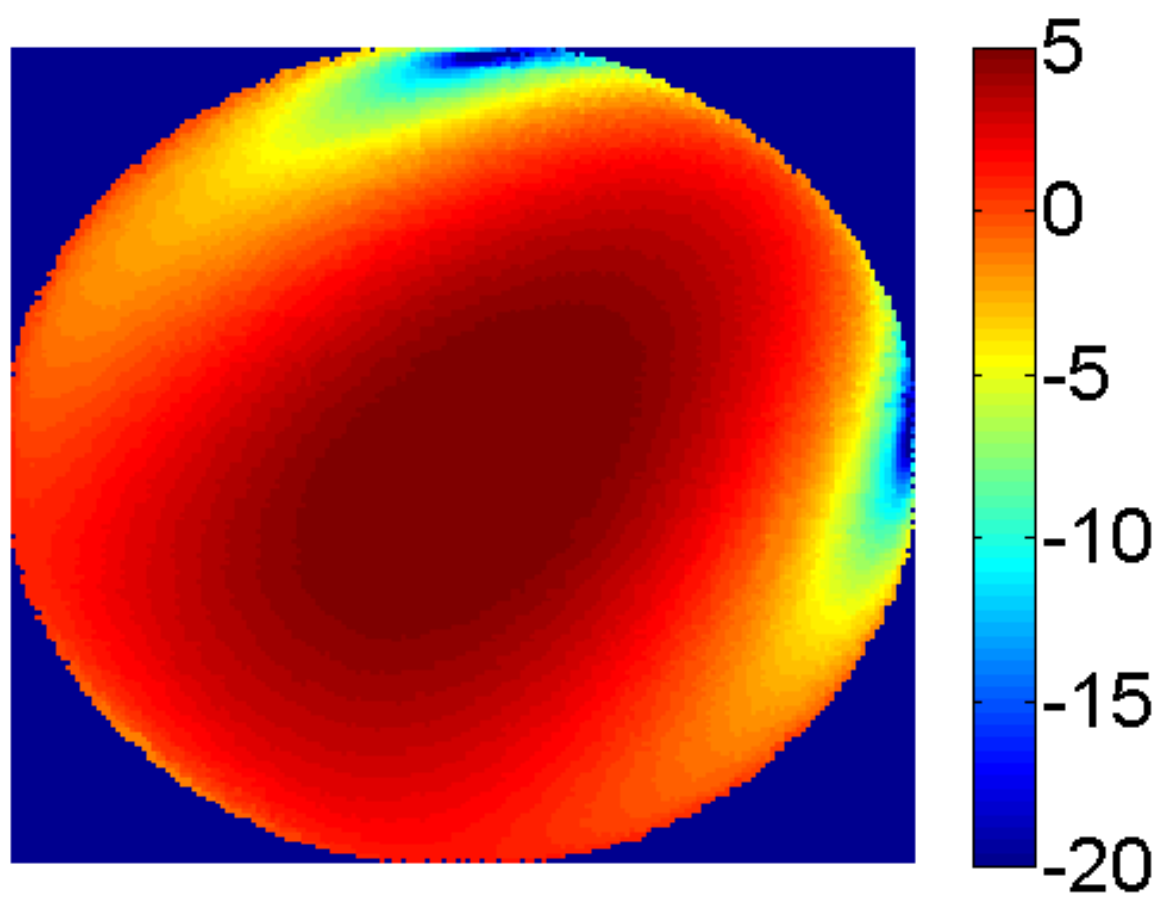

Figure 19 : OCSP Half Space CoPol Gain (dB)
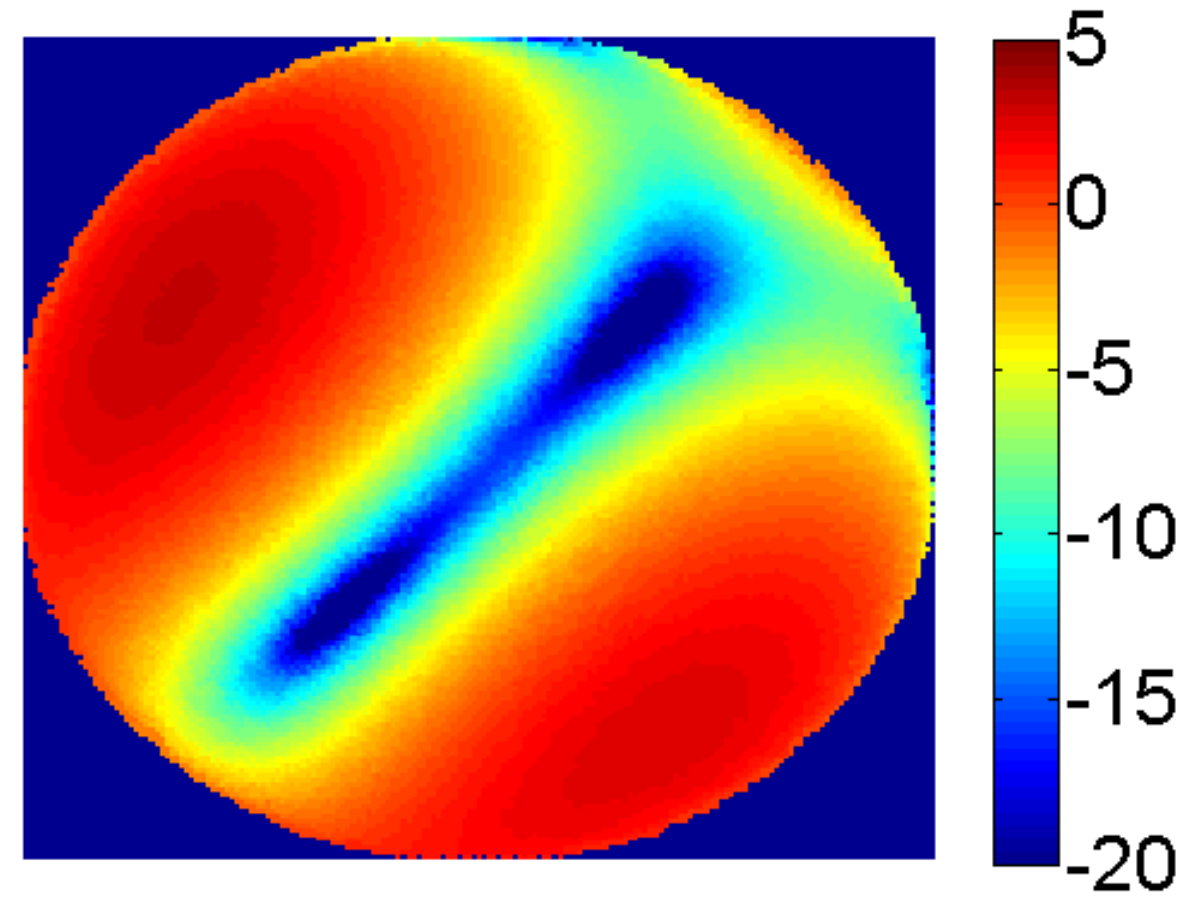

Figure 20 : OCSP Half Space XPol Gain (dB) 


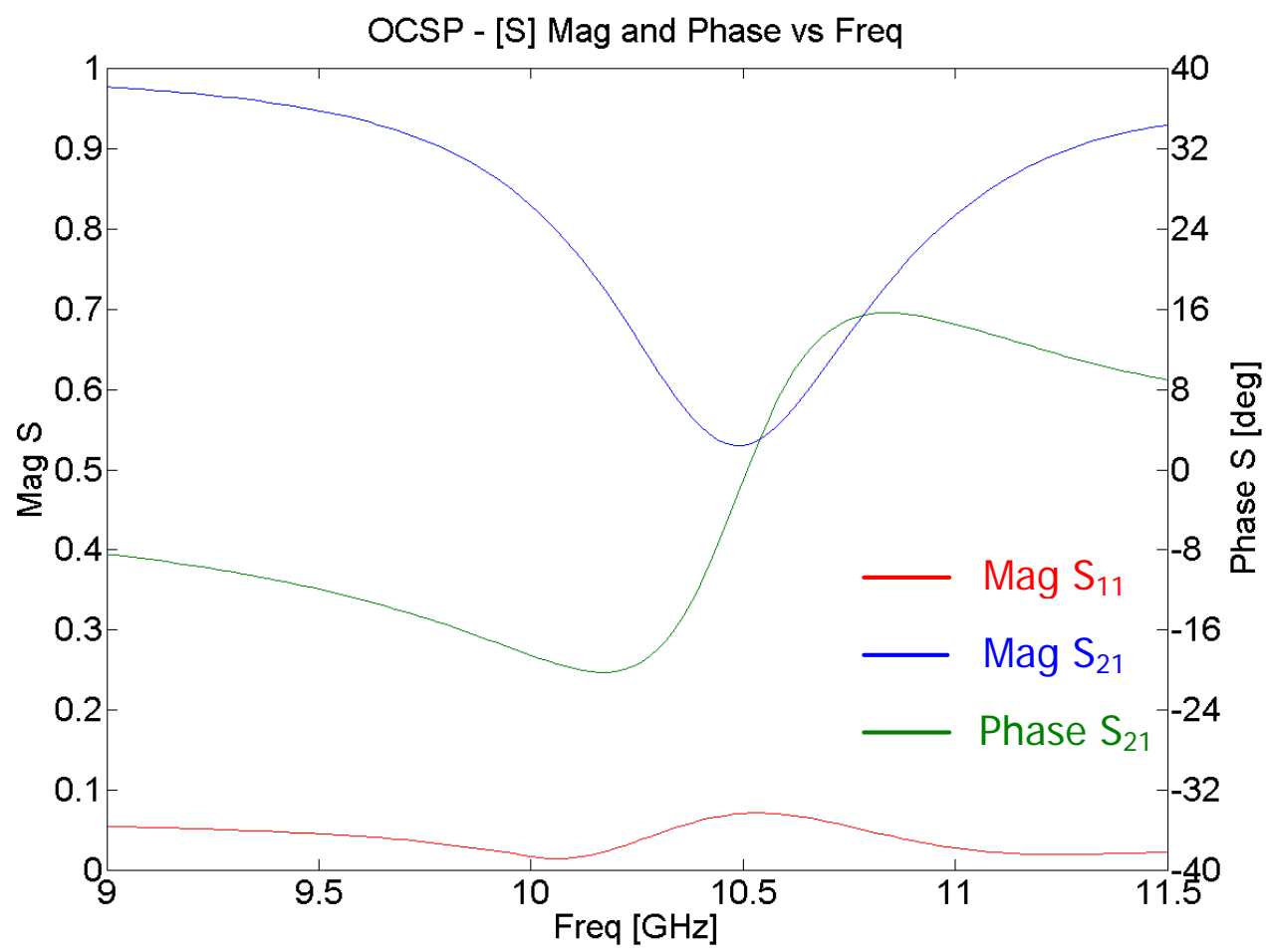

Figure 21 : OCSP S-Parameter Performance

OCSP S-parameters were also calculated and are presented in Figure 21. While the overall shape of each parameter was similar to the T-slot, the ranges were different. The $\mathrm{S}_{21}$ data changed more gradually with frequency and had a larger minimum. $\mathrm{S}_{11}$ was lower across the band, thus the OCSP also exhibited the requisite low return loss required for the traveling wave array design procedure.

\subsubsection{X-Slot}

As discussed above, the X-slot was the first CP slot radiator to which an entire technical journal paper was devoted. This original paper by Simmons was highly experimental, yet also provides the only published theoretical design guidelines to date. The proposed theory of operation is somewhat different for the X-slot than the T-slot or OCSP. Instead of focusing on establishing orthogonal slots whose aperture fields are in quadrature and of an equal magnitude, the focus was placed on the $\mathrm{TE}_{10}$ source fields. Equations (8) and (9) may be equated and solved for 
the positions of equal magnitude across the transverse dimension of the waveguide:

$$
x= \pm \frac{a}{\pi} \cot ^{-1}\left(\frac{\beta a}{\pi}\right)
$$

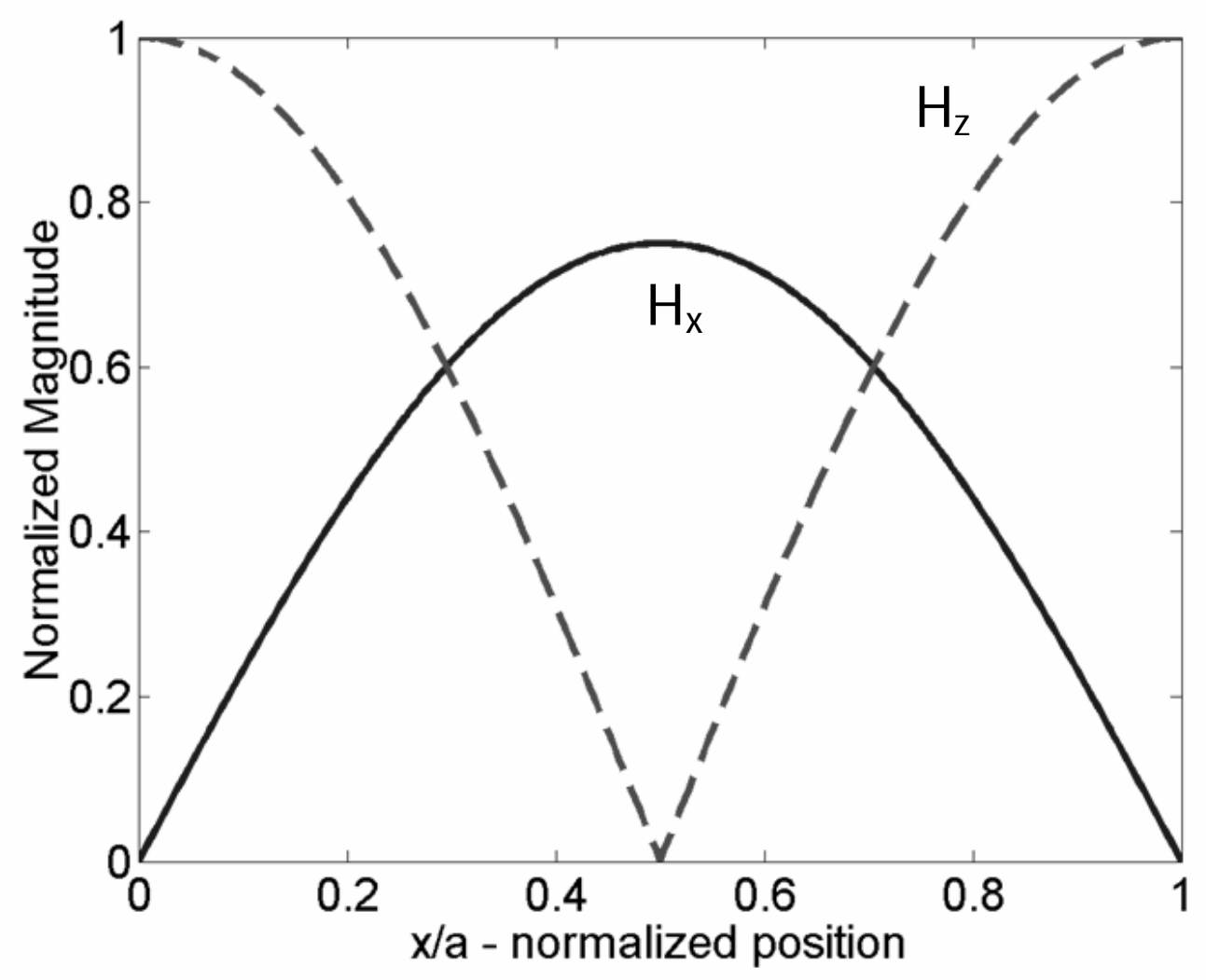

Figure 22 : Normalized Magnitude of $H_{x}$ and $H_{z}$

The normalized magnetic field values across the transverse dimension of a rectangular waveguide for a $\mathrm{TE}_{10}$ field are seen in Figure 22. The theory of operation put forth by Simmons was that any aperture placed in the broad wall of a waveguide at the positions described by (10) will produce $\mathrm{CP}$ radiation. That is, even a small circular hole will radiate $\mathrm{CP}$ at this position. Simmons confirmed these results, but also observed that an electrically large aperture was required to couple an appreciable amount of energy from the waveguide. While a small 
circular hole was an interesting example, it served little practical purpose. According to his paper, he pursued a pair of orthogonal linear slots as a more useful $\mathrm{CP}$ radiator, based on suggestions from Watson. He found that due to the offset required by (10), the slots had to be rotated in order to fit a full $\lambda / 2$ slot entirely within the broad wall face.

An alternative theory of operation is to consider two compound slots having the same offset but opposite rotation angles. In this way the X-slot and OCSP may be thought of as conceptually identical. An investigation to this theory of operation has been carried out and was found to predict the relationship between $\mathrm{X}$-slot $\mathrm{AR}$ and $\mathrm{S}_{11}$ performance. The details of this investigation are beyond the direct scope of this work and may be found in Appendix A.

An HFSS simulation similar to those above was carried out for an X-slot radiator. Calculated radiation patterns of this simulation are seen below in Figure 24 and Figure 25, for Ports 1 and 2, respectively.

The $\mathrm{CP}$ gain patterns exhibited strong similarities with the T-slot. Symmetry about zenith and the anticipated switch of CP sense with a shift in port excitation were observed. The axial ratio value is only $3 \mathrm{~dB}$, but, as will be discussed in Chapter 5, this can be improved through a few simple design refinement steps. 


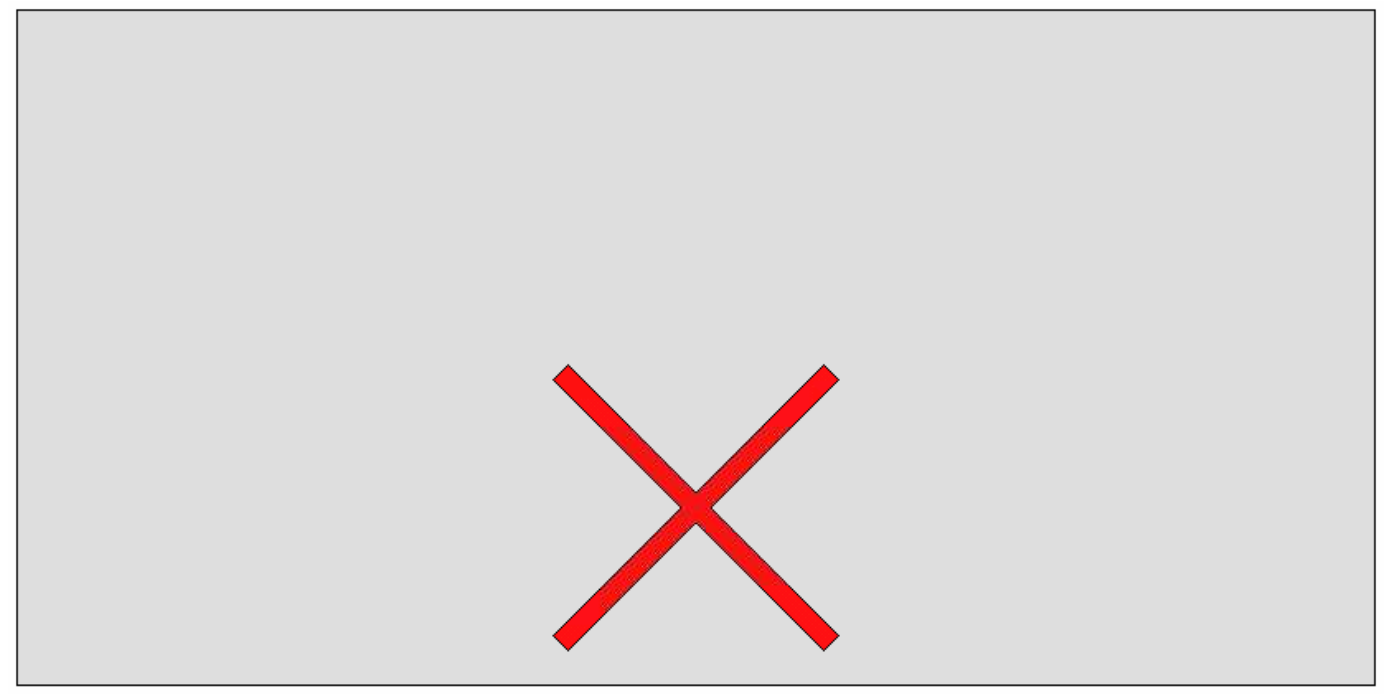

Figure 23 : Simulated X-Slot

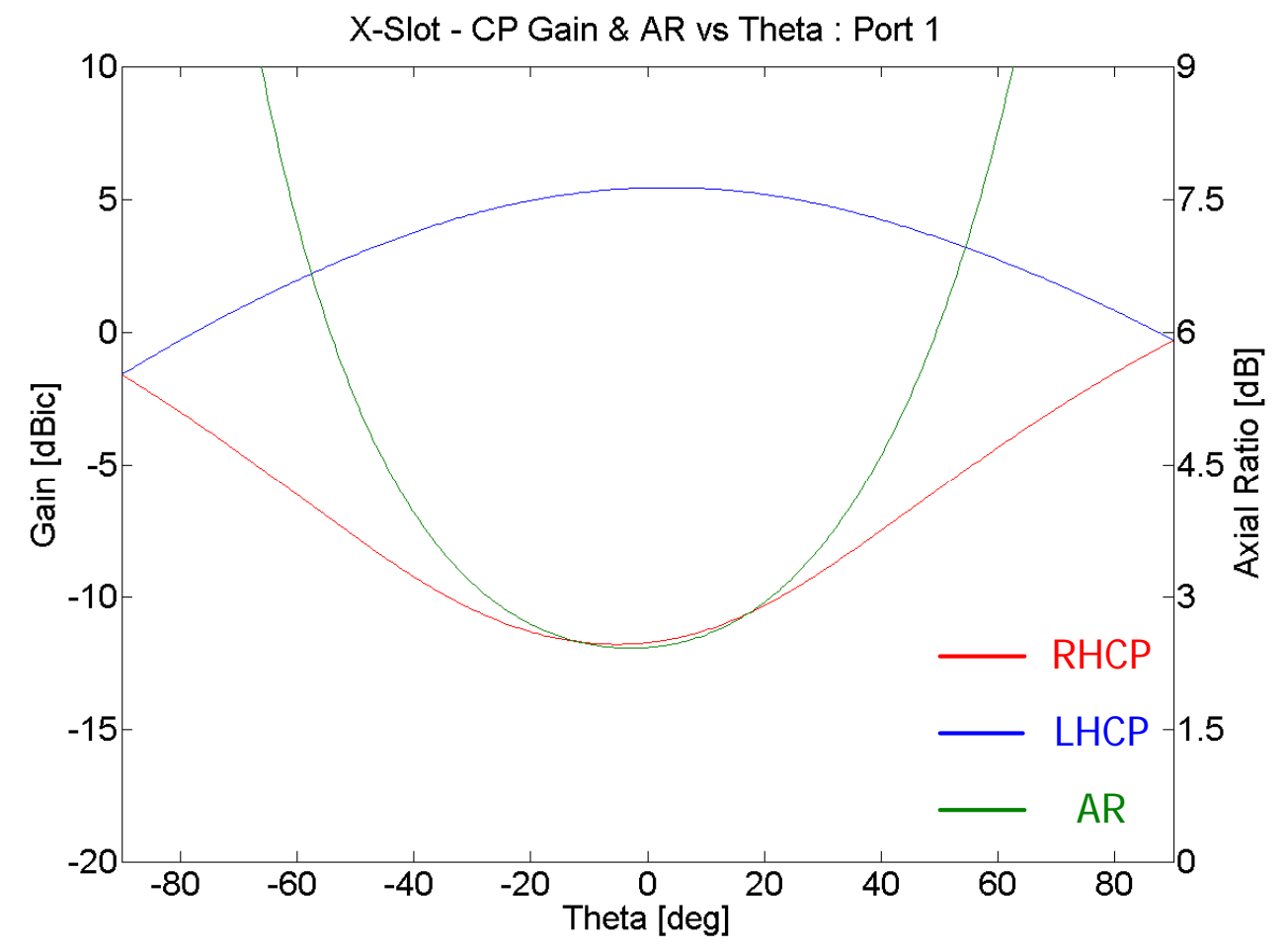

Figure 24 : X-Slot LHCP Pattern 


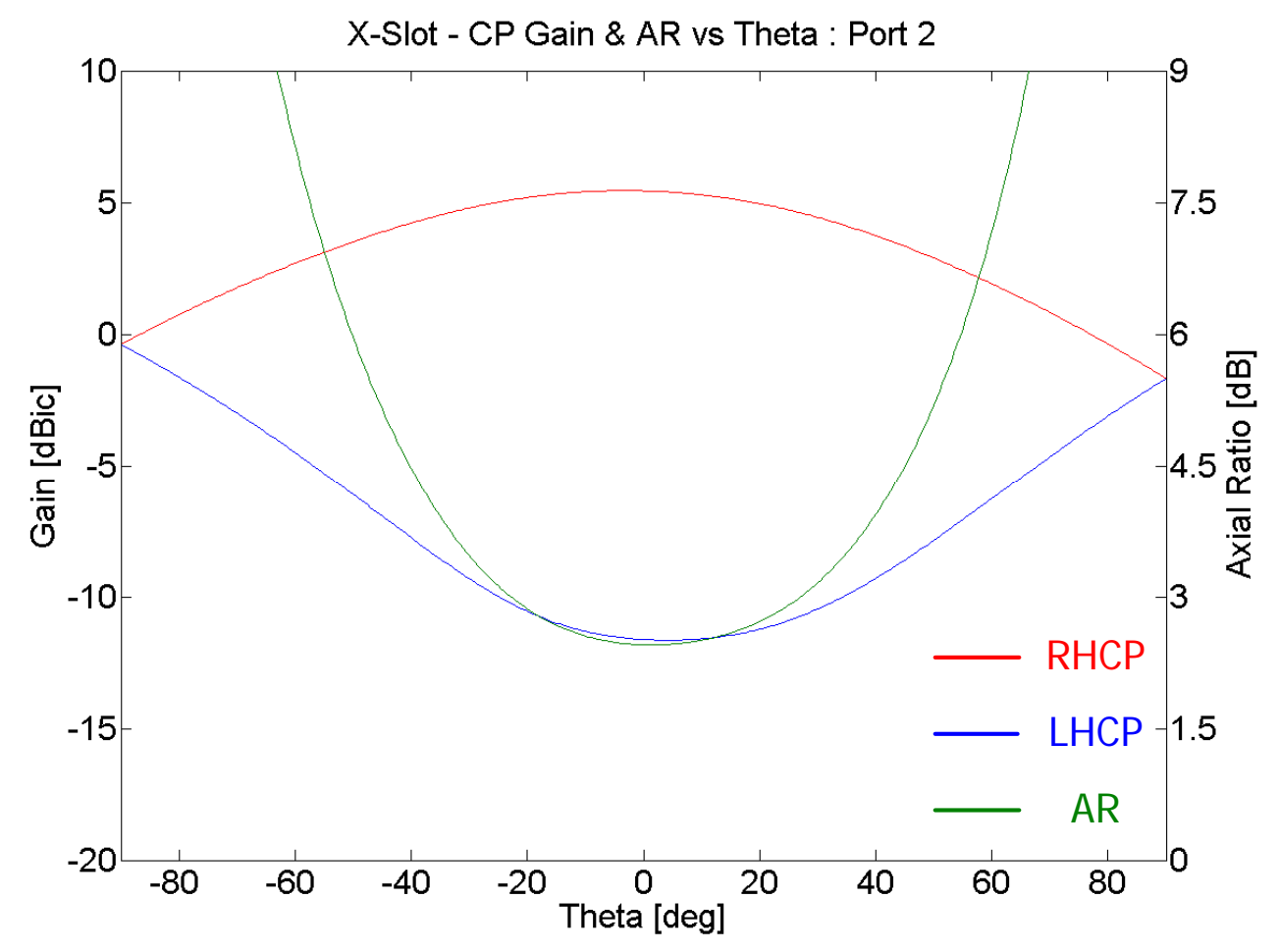

Figure 25 : X-Slot RHCP Pattern

An expanded view of the radiation patterns are captured in Figure 26 and Figure 27 where, as with the T-slot and OCSP, the gain was calculated over the entire upper hemisphere and projected onto a circle. The array factor anomalies observed with the other two CP slot configuration simulations was absent. If one considers the X-slot theory of operation of two, co-located compound slots of opposite rotation angles, these results come as no surprise. The phase center of each slot is at the same point, thus no pattern distortion caused by a unit cell array factor would be expected.

Finally, the S-parameter performance of the X-slot unit cell was calculated and is presented in Figure 28. The $\mathrm{S}_{21}$ magnitude and phase responses were nearly identical to the OCSP while the $S_{11}$ response was slightly improved, on average, across the band. Thus, the return loss was low enough to employ a traveling wave array design approach with an X-slot array. 


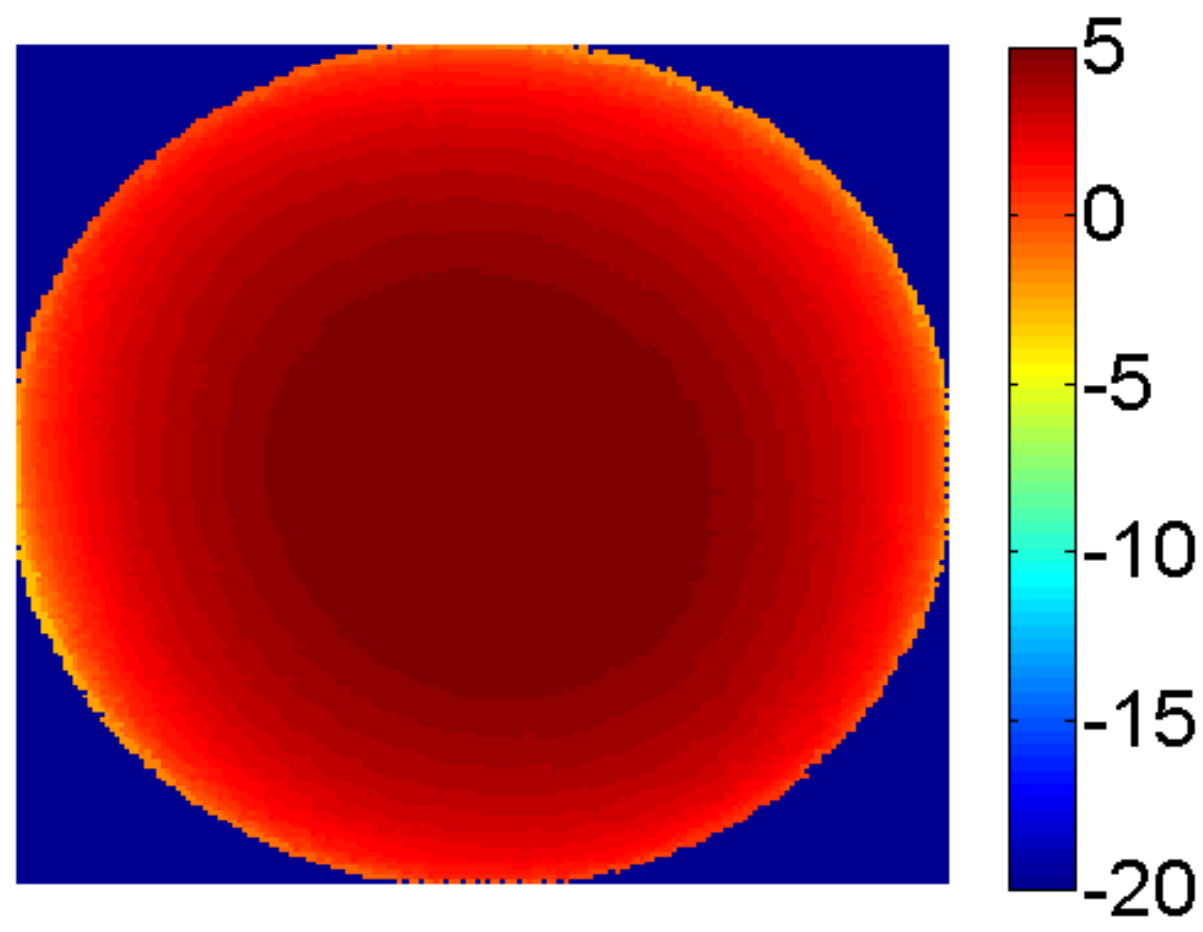

Figure 26 : X-Slot Half-Space CoPol Gain (dB)

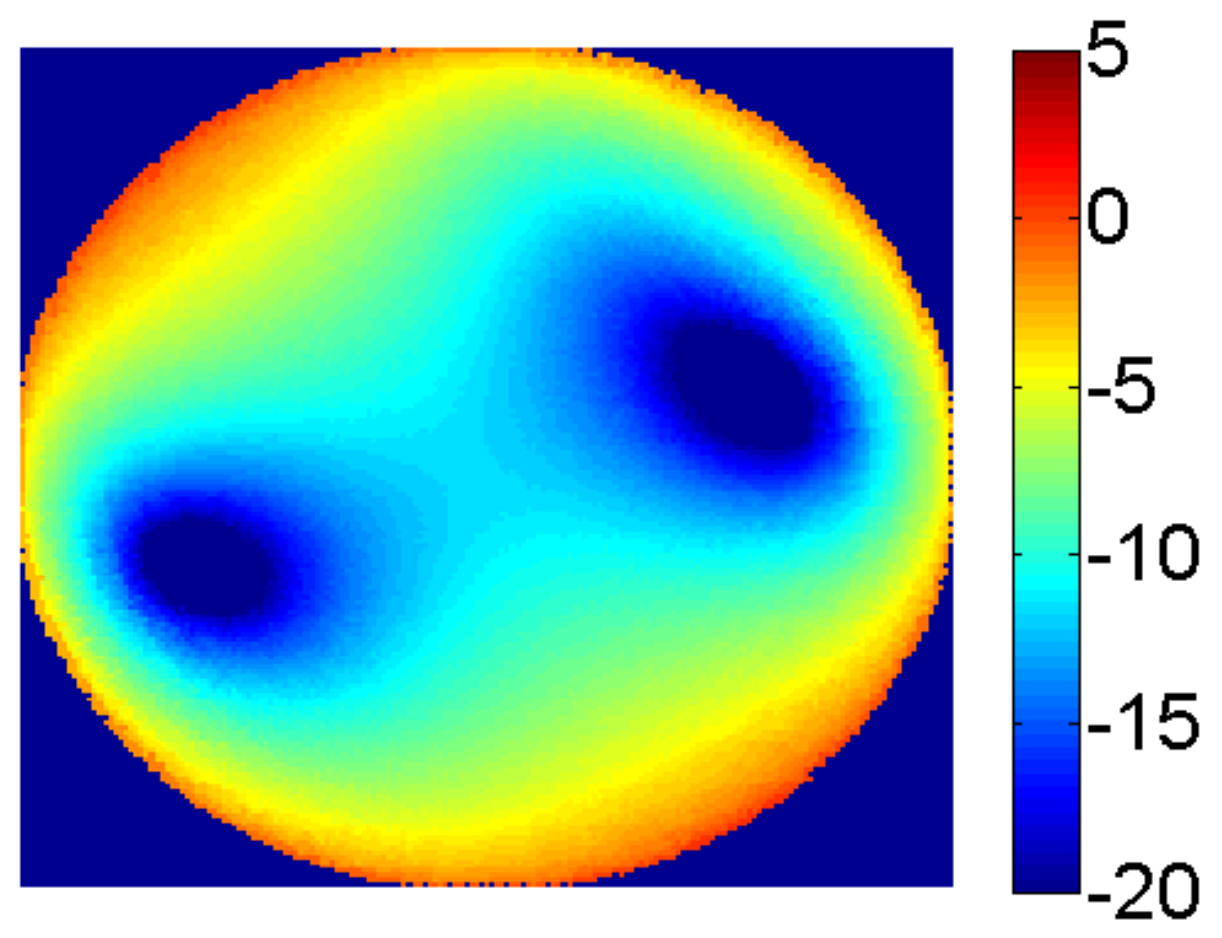

Figure 27 : X-Slot Half-Space XPol Gain (dB) 


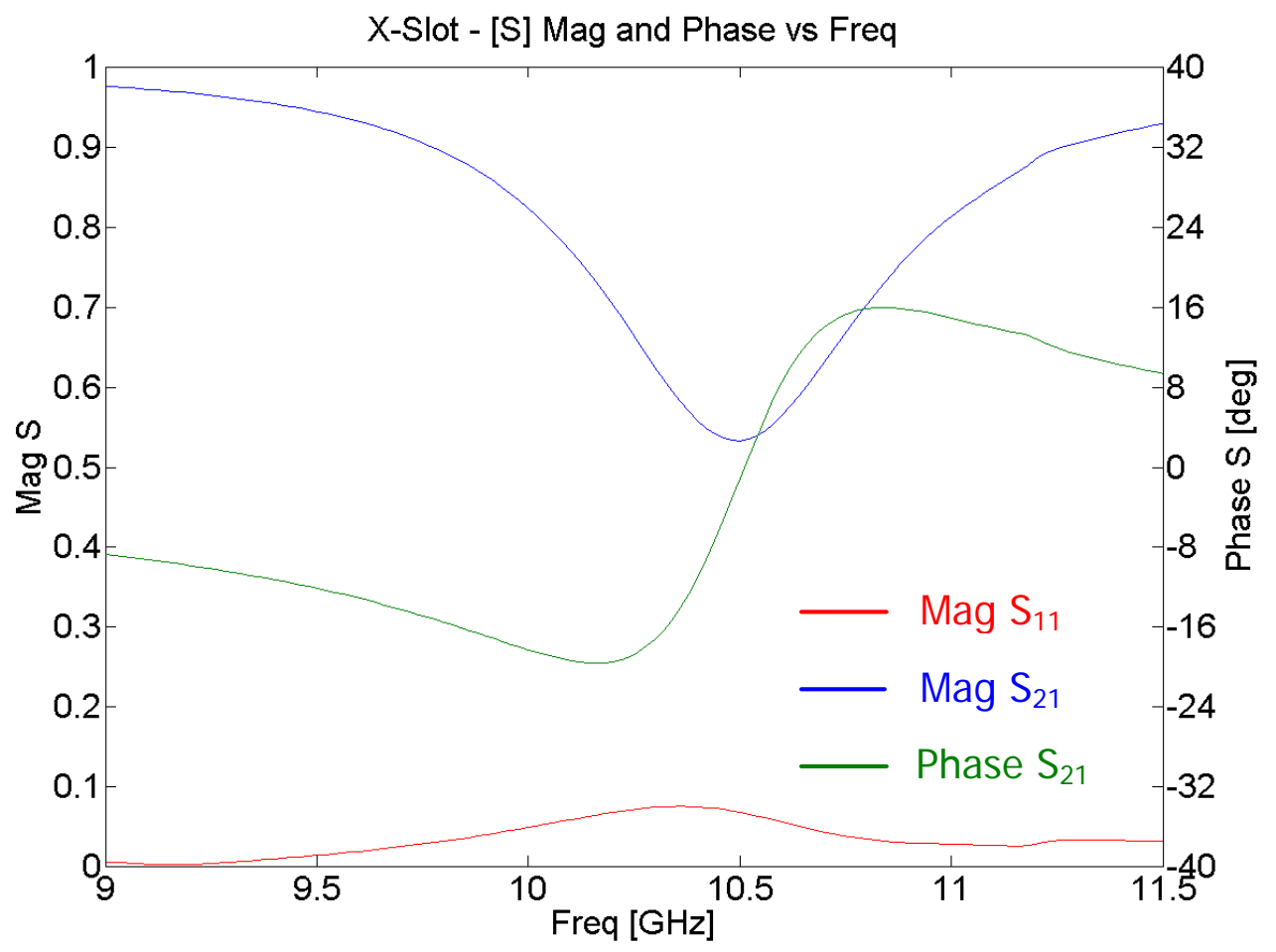

Figure 28 : X-Slot S-Parameter Performance

\subsubsection{Radiator Down Selection}

From the above simulation data, it was apparent that the CP slot alternatives all perform similarly. The peak directivities are almost identical, the AR patterns are similar, and the only radiation pattern differences can be understood in terms of the phase center separation of the T-slot and OCSP sub-slots. In this regard, with all other radiation characteristics being equal, the X-slot has a slight advantage due to its pattern symmetry, but it must be acknowledged that any of the slots could suffice.

The X-slot and OCSP scattering parameter performances were almost identical with both showing a sufficiently small return loss and range of radiated coupling values to be useful in a traveling wave array design. The T-Slot return loss was slightly worse and maintained a steeper response with frequency, which would translate to tighter mechanical tolerance requirements in a manufacturing 
setting. Over the range of typically useful $\mathrm{S}_{21}$ values - i.e. between 0.95 and 0.7 (a justification for this range will be provided below) - the X-slot had a consistently lower return loss than the OCSP, thus the choice was slightly in favor of the X-slot.

For the purposes of this work, a single CP slot topology was required. With the performance of all three slots being fairly equal, the X-slot was chosen. It is acknowledged, however, that the T-slot and OCSP could probably function adequately in the array topology. Developing design approaches for the OCSP and T-slot is left for a future endeavor.

\subsubsection{The Phase Shifter}

The selection of a phase shifter to reduce inter-element spacing for in-phase excitation of a linear subarray of waveguide slot radiators was more straightforward than the selection of a CP slot topology. Due to the previously discussed non-linear relationship between feed loss and G/T performance of satcom systems (and the generalized negative impact of feed loss), the majority of active phase shifting elements were immediately ruled out. The remaining alternatives included loading the waveguide with low loss dielectric and periodically loading the waveguide with reactive obstacles to produce a slow wave structure. Both techniques are well known to increase the guided wavenumber - i.e. decrease $\lambda_{\mathrm{g}}$ - and both techniques offered the possibility of discrete or continuous loading [2-5 and 3-4 - 3-7]. Figure 29 depicts these alternatives in cross section. 


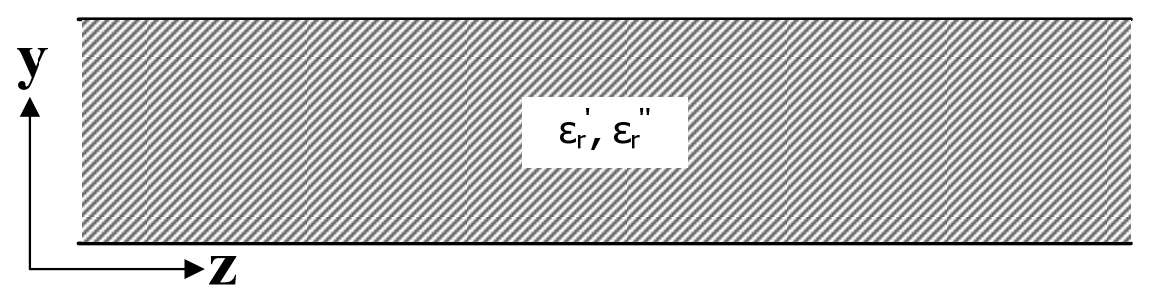

(a) Continuous Dielectric Loading

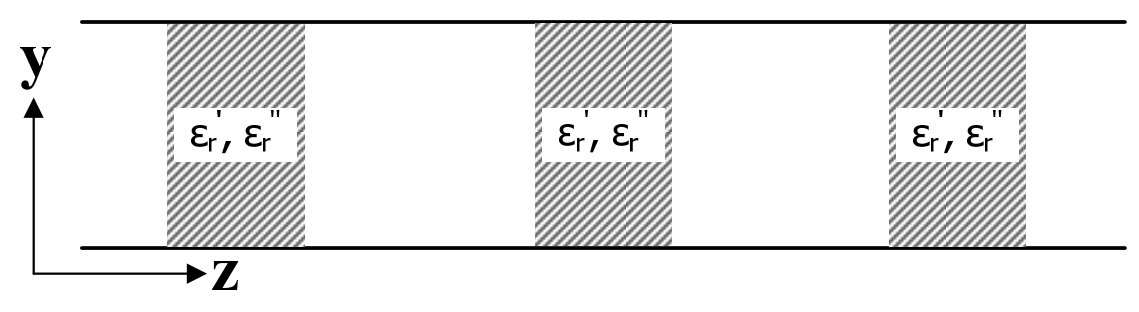

(b) Discrete Dielectric Loading

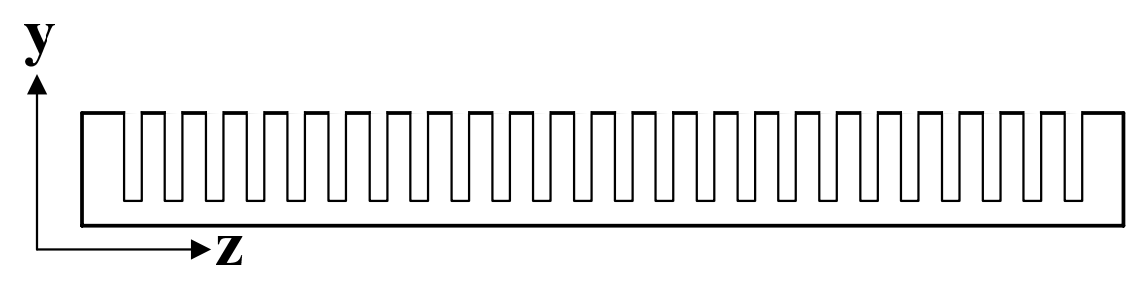

(c) Continuous Slow Wave Structure Corrugated Loading

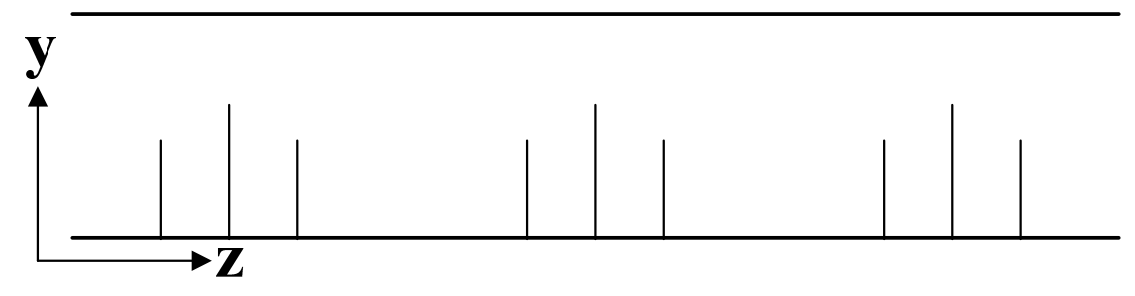

(d) Discrete Slow Wave Structure Iris Loading

Figure 29 : Passive Waveguide Phase Shifters

\subsubsection{Phase Shifter Loss}

Down-selection criteria for the passive phase shifter alternatives can be generated from theoretical expressions related to waveguide loss and design approach considerations. This section will address the issue of phase shifter loss while the 
following will tackle the unique design problems associated with each type of waveguide passive phase shifter.

The conduction loss experienced by a $\mathrm{TE}_{10}$ mode propagating through a metallic rectangular waveguide filled with an arbitrary dielectric may be expressed as follows:

$$
\alpha_{c}=\frac{R_{s}}{b \eta \sqrt{1-\left(\frac{\lambda}{\lambda_{c}}\right)^{2}}}\left[1+\frac{2 b}{a}\left(\frac{\lambda}{\lambda_{c}}\right)^{2}\right]
$$

If dielectric loss is included, the total loss experienced is increased by an amount approximately equal to

$$
\alpha_{d} \approx \frac{\varepsilon_{r}^{\prime \prime}}{\varepsilon_{r}^{\prime}} \frac{\pi \lambda_{g}}{\lambda^{2}},
$$

where $\mathbf{R}_{\mathbf{s}}$ is the surface resistance of the waveguide metallic walls; $\mathbf{b}$ is the waveguide height; $\lambda$ is the wavelength in an unbound medium of equivalent permittivity and permeability as that filling the waveguide; $\lambda_{c}$ is the cutoff wavelength of the $\mathrm{TE}_{10}$ mode; $\boldsymbol{\varepsilon}_{\mathbf{r}}$ is the real part of the relative permittivity for the medium filling the waveguide; $\boldsymbol{\varepsilon}_{\mathbf{r}}$ " is the corresponding imaginary part of the relative permittivity; and $\boldsymbol{\eta}$ is the impedance of the medium filling the waveguide. The total loss per unit length is the algebraic sum of (11) and (12).

From (11) and (12) it is seen that in addition to weight and cost considerations, dielectric loading will increase the average conduction and dielectric loss of a waveguide. While the two equations only apply strictly to the case of a uniform dielectrically loaded waveguide, the increase in loss accompanying a waveguide loaded with discrete dielectric plugs will be larger than that of an air-filled waveguide.

The task of determining the conduction loss from an air filled rectangular waveguide loaded with slow wave structures is a considerable challenge. A number of simple simulations were performed in HFSS to determine an approximate relationship between conductor loss in an air filled waveguide and 
one periodically loaded with capacitive irises or corrugations. It was found the amount of conductor loss scaled roughly with twice the ratio of the slow wave structure wave number to the original waveguide wave number. If this approximation is assumed, then the following inequality may be stated regarding the required material dielectric loss tangent to match the loss performance of a slow wave structure passive phase shifter (see Appendix B for the full derivation):

$\tan \delta<\frac{1}{\varepsilon_{r}} \sqrt{\frac{2}{\omega \mu \sigma}}\left(\frac{2}{b}\left(\frac{\beta_{c}}{\beta_{0}}\right)^{2}-\frac{\sqrt{\varepsilon_{r}}}{b^{\prime}}\right)\left[1+\frac{b}{2 a^{3}}\left(\frac{c}{f}\right)^{2}\right]$

In (13), $\boldsymbol{\beta}_{\mathbf{c}}$ is the slow wave structure wave number; $\boldsymbol{f}$ is the frequency of operation; $\boldsymbol{\varepsilon}_{0}$ is the permittivity of free space; and $\boldsymbol{\sigma}$ is the conductivity of the metal comprising the waveguide walls.

For a copper X-band waveguide operating at $10 \mathrm{GHz}$ with an increase in the guided wave number of about 1.5, a dielectric loading material would require a loss tangent of around $10^{-4}$ to compete with the slow wave structure passive phase shifter. Dielectric materials of this caliber are not unheard of at X-band, but they are almost exclusively ceramics, [3-8] which will translate to a greater increase in system weight than the more common polymer based dielectrics would cause. Additionally, loading a waveguide with ceramic dielectrics presents machining difficulties which exceed that of a milling operation required to construct a waveguide loaded with slow wave structure irises.

\subsubsection{Design Challenges}

Each type of waveguide passive phase shifter presents a unique set of design difficulties. This section will consider each in turn.

\section{Approach 1: Continuous Dielectric Loading}

Pro: Simple to analyze from classic waveguide equations. $\lambda$ compresses by the square root of $\varepsilon_{\mathbf{r}}$, thus a permittivity value may be easily found which provides $\lambda_{\mathbf{g}}$ $<\lambda_{0}$ to prevent grating lobes and allow broad side radiation. 
Con: $\lambda_{c}$ must be adjusted to ensure the next mode of operation does not exist when the waveguide is sufficiently loaded. Satisfying the two equations simultaneously is simple, but will necessitate shrinking the width of the waveguide to maintain single-mode operation. This restricts the area available for slot radiators, making the placement of a resonant slot entirely within the broad face of a rectangular waveguide impossible. Additionally, the permittivity discontinuity existing between the waveguide interior and free space perturbs the performance of the slot in a manner that is difficult to characterize.

\section{Approach 2: Discrete Dielectric Loading}

Pro: Transmission line analysis of cascaded elements can be employed to determine the amount of required phase shift from each block. As with the continuously distributed dielectric loading approach, simple equations are available to calculate the required permittivity.

Con: In order to maintain single-mode operation and an impedance match with unloaded portions of the waveguide, the waveguide width and height will have to be shifted at the location of the dielectric loading. This only slightly complicates the design procedure, adding another inequality constraint to the optimization process; however, the manufacturing challenges presented by such an operation are equal to or greater than those associated with slow wave structure loading.

\section{Approach 3: Continuous Iris/Corrugation Loading}

Pro: Design equations have been in existence for over fifty years [2-5] and the basic corrugated waveguide structure is widely used (in horn antenna applications, for example) and well understood.

Con: The slow wave structure passive phase shifter will, by design, perturb the driving sources throughout the waveguide region. If a slot radiator is placed in a wall directly opposite the slow wave structure, the slot will not operate as if it were in an unloaded waveguide. Thus, the design of the radiating elements and phase shifters are inextricably linked. This coupled design problem is much more challenging than the discrete slow wave structure problem. Additionally, if the 
slots comprising the linear subarray are not all identical, their relative forward scattered and radiated phase responses will have to be accounted for in the phase shifter design. This will be a much more challenging problem if a continuously distributed slow wave structure phase shifter is utilized.

\section{Approach 4: Discrete Iris Loading}

Pro: The design procedure may be broken into independent unit cells. The forward scattered phase response of each unique slot radiator can be easily addressed. Fewer irises than the continuously distributed version implies a less expensive manufacturing process.

Con: Not well understood and no design equations exist.

\subsubsection{Phase Shifter Down Selection}

Despite the lack of a design approach, the discretely loaded iris slow wave structure passive phase shifter approach offers the most manufacturing and technical benefits of the four choices. The goal of the next chapter is to address the lack of a design approach and develop a reliable, robust, efficient, and widely applicable design methodology.

\subsection{The Design Approach}

Having addressed the problems of selecting a radiating element and a passive phase shifter topology, the task remains to select a design approach. That is, if the goal of the following chapters is to develop a design methodology for the slow wave structure phase shifter and X-slot radiator, respectively, then overall array design approach must be established to determine which aspects of device performance require characterization.

A few of the design approach goals have been previously stated - that is, in order to maintain good $\mathrm{CP}$ radiation, a traveling wave design approach must be utilized, and the technique must be widely accessible. Thus, developing a set of 
specialized, efficient MoM codes to simulate an X-slot radiator and a discrete slow wave structure passive phase shifter would ultimately prove inefficient to the vast majority of designers.

\subsubsection{The Aperture Distribution}

A very important problem faced by every array designer is the aperture distribution. That is, the set of relative amplitude and phase relationships describing the radiating fields of each element in the array must be determined, relative to the known performance requirements. In the case of the dual-CP waveguide slot array with broadside radiation, which is at the focal point of this work, half of the problem has already been defined. That is, the inter-element phase shift must be as close to zero as possible to ensure the main beams associated with each sense of CP do not diverge.

The problem of an aperture amplitude distribution is somewhat more complicated. Traditional traveling wave array designs, like Dion's method, have been predicated on the notion of one feed direction and one load termination point to minimize reflections. The design methodology need not be stated in full here, but can be summarized by the following equation [1-10, 3-9, and 3-10]:

$$
g_{i}=\frac{P_{i}}{1-\sum_{n=1}^{i-1} P_{n}}
$$

In (14), $\mathbf{g}_{\mathbf{i}}$ refers to the normalized element conductance; $\mathbf{P}_{\mathbf{i}}$ is the radiated power by element $\mathbf{i}$; and the denominator indicates the amount of power available to the slot. Dion's method assumes the use of non-resonant, well-matched slots so the total amount of power available in the low loss feed line at any given slot can be calculated by summing the power radiated through all the slots between it and the source. The steps in this methodology can be summarized as:

1. Determine the normalized aperture distribution.

2. Calculate the amount of power to be terminated in the load $\left(\mathrm{P}_{\mathrm{N}}\right)$. 
3. Solve for $\mathrm{g}_{\mathrm{N}-1}, \mathrm{~g}_{\mathrm{N}-2}$, etc. until all the normalized conductance of each slot has been calculated.

While this method and slight evolutionary variations have been used for the previous 50 years with good success, the problem of a traveling wave array that is fed from both ends is not addressed. If a traveling wave array is designed to be fed from one end and terminated in a matched load at the other end, the array performance will not be identical if the matched load and feed are reversed.

In the three figures which follow, this effect in a typical traveling wave linear array is considered. First, in Figure 30, the normalized slot conductance of each element is captured. Next, the calculated array performance is presented for two setup variations. The design case is presented (referred to as "Port 1"), as well as the scenario where the source and matched load are reversed (referred to as "Port 2").

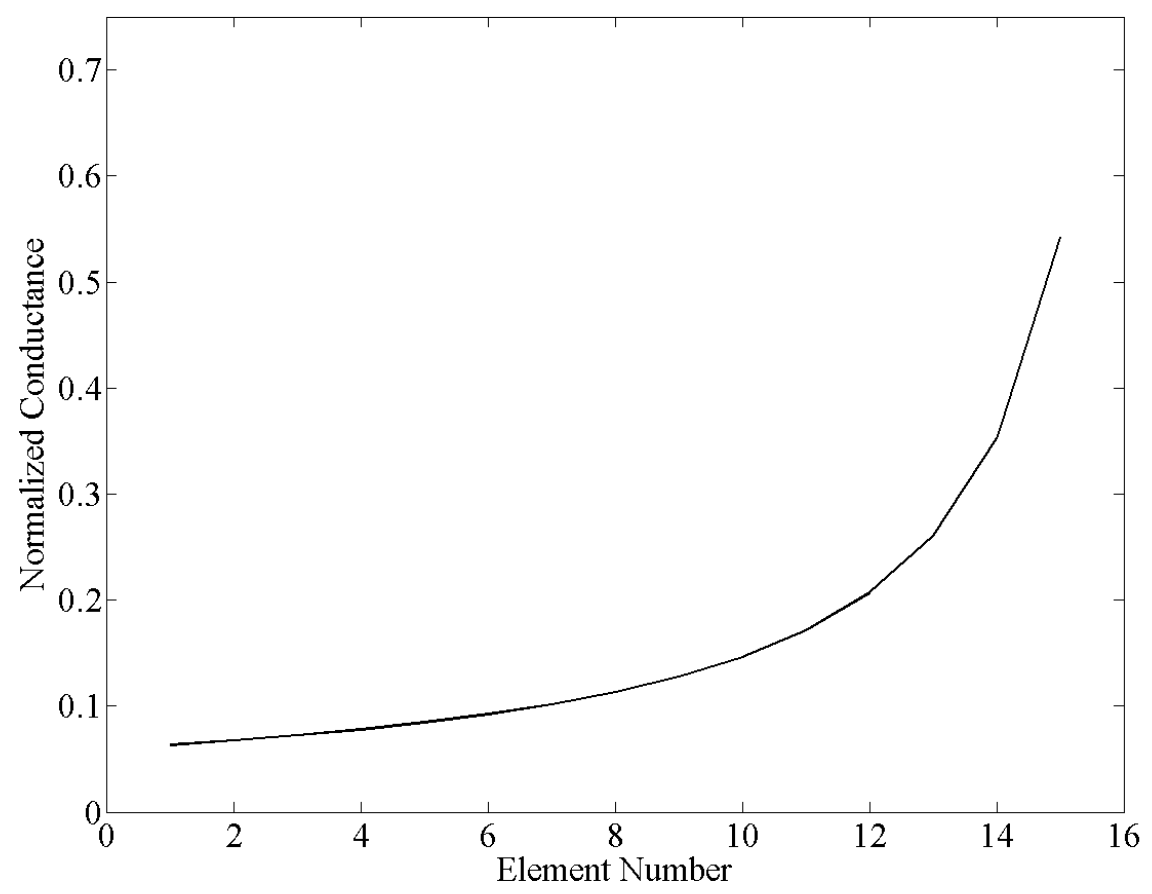

Figure 30 : Traveling Wave 15-Element Linear Array Slot Conductance 


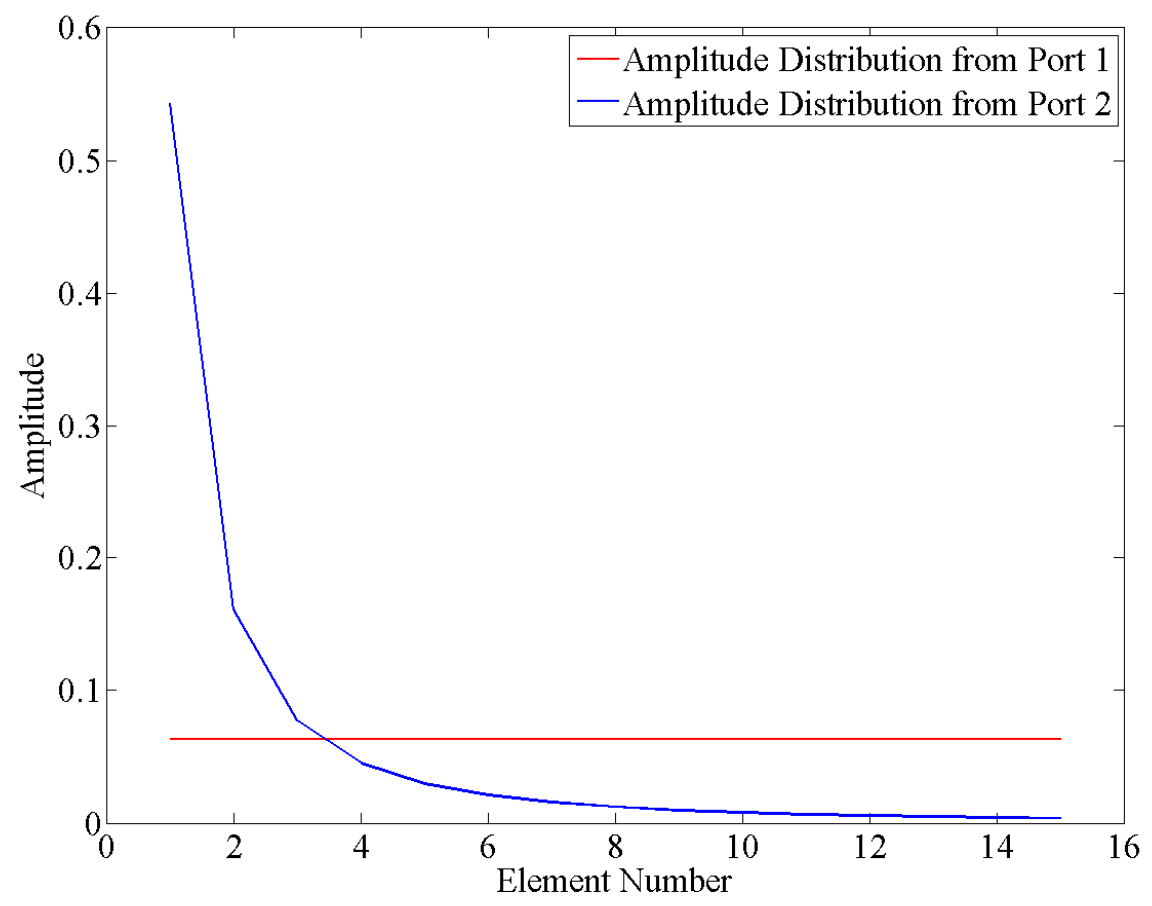

Figure 31 : Traveling Wave Linear Array Aperture Power Distribution

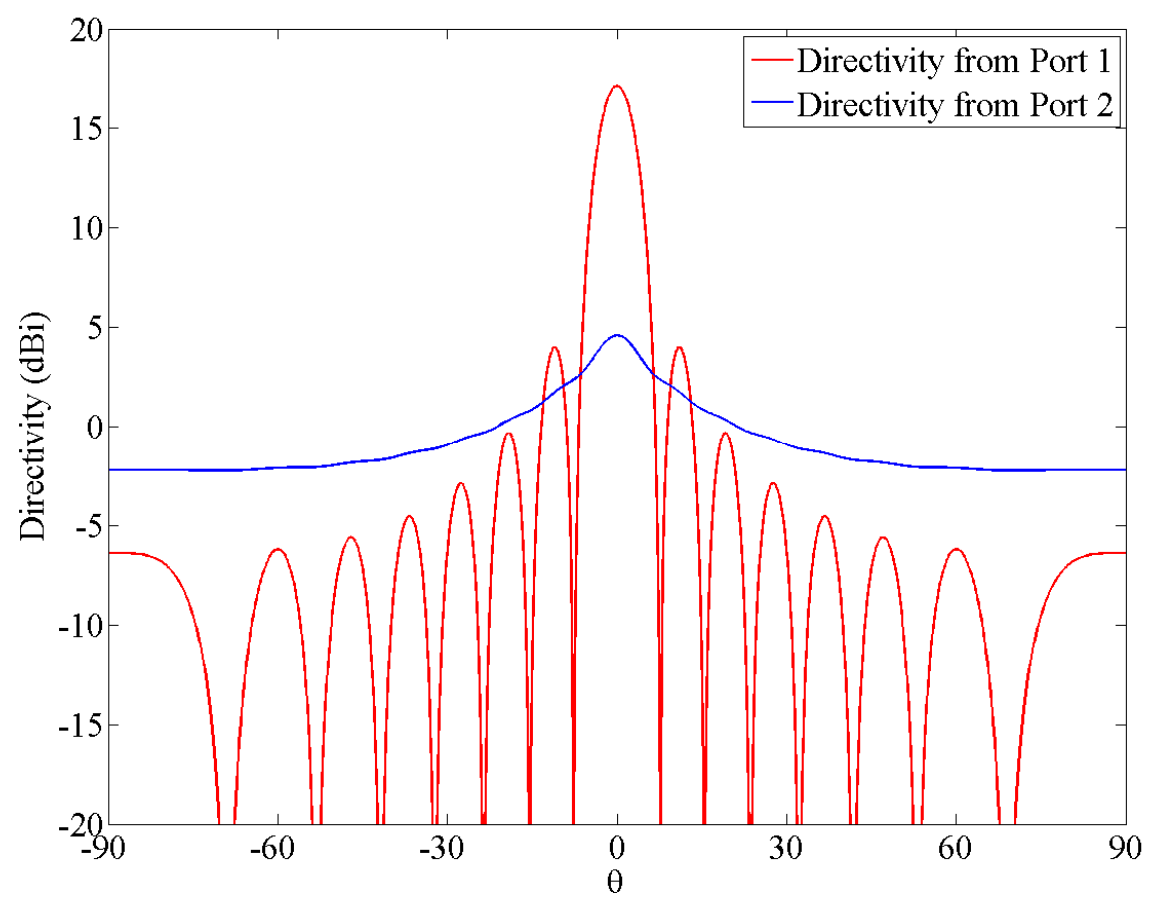

Figure 32 : Fwd and Rev Fed Traveling Wave Linear Array Directivities 
In Figure 31, the aperture power distribution is observed, where the magnitudes are referenced to a unity source. The resultant far fields from each aperture distribution are calculated in Figure 32. From the plot, it is quite clear that standard traveling wave array design techniques are unacceptably asymmetric and inefficient in a dual-polarization setting.

The problem of determining an ideal distribution to maximize gain when fed from either end of a traveling wave array has been addressed by Sakakibara, et al in 1999 [2-1]. A Calculus of Variations approach was taken whereby a theoretically optimal set of element coupling ratios was calculated and related to the amount of energy terminated in the load. This was then compared with a purely uniform set of coupling ratios (i.e. every slot being identical), the results of which are re-created in Figure 33.

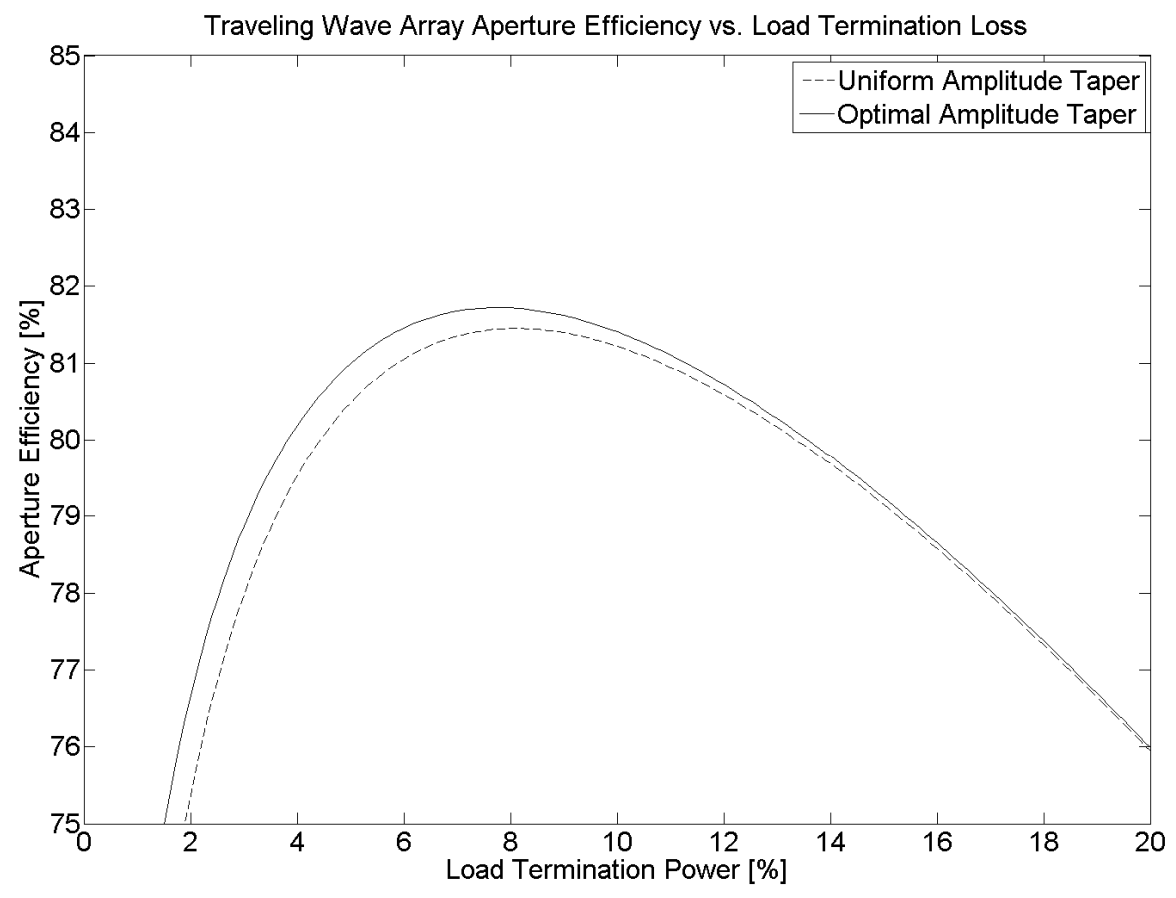

Figure 33 : TW Aperture Efficiency Comparison

Four points of interest appear from the results of Sakakibara's work. First, the optimal and uniform coupling distributions provide nearly identical aperture efficiencies. Second, the maximum aperture efficiency appears to be around $82 \%$. 
Third, a wide range of load terminations should provide aperture efficiencies above $80 \%$. Finally, the aperture efficiency can be equated to the load termination power - a simple quantity to calculate.

Taking advantage of these results, the following design approach is suggested for dual-CP waveguide slot arrays.

1. Every slot in any given linear subarray should be identical and have a good axial ratio (i.e. $\mathrm{AR}<3 \mathrm{~dB}$ )

2. The slot should be well-matched (i.e. $\mathrm{S}_{11}<0.1$ )

3. Unit cell simulations should be performed to design the slot for a desired $\mathrm{P}_{\mathrm{rad}}$ and $\mathrm{S}_{21}$

4. The forward scattered phase perturbation of the slot should be accounted for, in addition to the inter-element spacing to determine the necessary phase shift from a slow wave structure.

5. Unit cell simulations should be performed on the slow wave structure to design/optimize against return loss $\left(\mathrm{S}_{11}<0.1\right)$ and phase shift.

It is further proposed that, for an N-element array, the following relationship may be used to calculate $\mathbf{t}$, the amount of power terminated in the load, based on the forward-scattered power from the X-slot radiator unit cell:

$$
t=S_{21}^{2 N}
$$

Thus, it is a trivial matter to solve for the required $S_{21}$ to achieve the desired $\mathbf{t}$, as taken from Figure 33, above:

$$
S_{21}=t^{\frac{1}{2 N}}
$$




\section{Chapter 4: Slow Wave Structure Passive Phase Shifters}

\subsection{Introduction and Definition}

A slow wave structure may be defined as any transmission line structure with a phase velocity slower than that of free space. Due to the reciprocal nature of the phase velocity and wave number, it may also be defined in terms of supporting a wave number larger than that of free space.

$$
v_{p}=\frac{\omega}{\beta}
$$

By contrast, waveguide modes have phase velocities larger than that of free space. Accordingly, they may be termed fast wave structures, although this term is not commonly used.

A typical dispersion diagram for a slow wave structure may be seen in Figure 34. In the plot, $\boldsymbol{\beta}_{\mathbf{c}}$ is the wave number for a slow wave structure formed by placing capacitive obstacles in a waveguide; $\boldsymbol{\beta}_{\mathbf{g}}$ corresponds to the wave number for the waveguide without the slow wave structure; and $\mathbf{k}_{\mathbf{0}}$ corresponds to the wave number in an unbounded medium. 


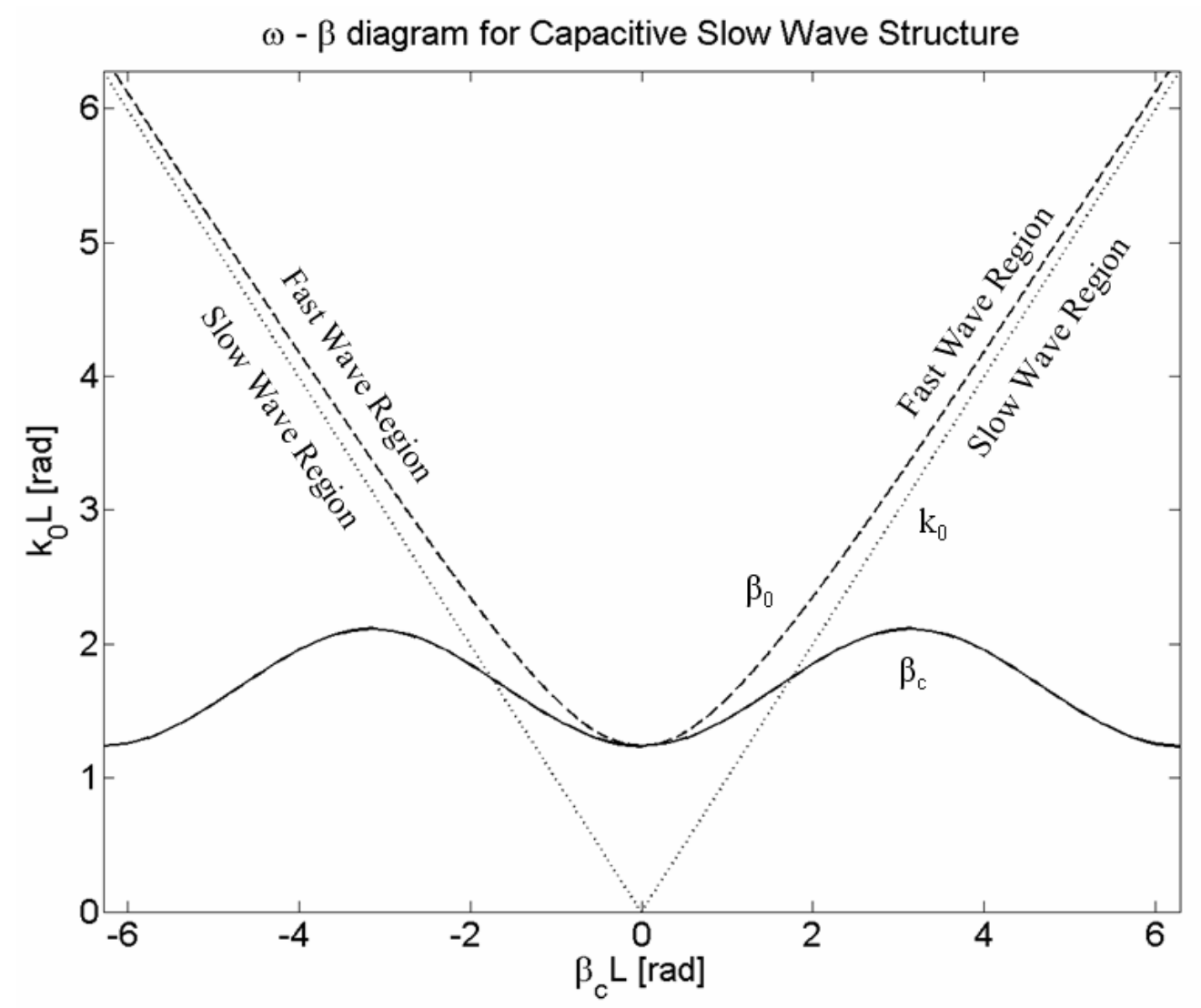

Figure $34: \omega-\beta$ Diagram for SWS and WG

The Fast Wave and Slow Wave regions are divided by the $\mathbf{k}_{\mathbf{0}}$ curve, also known as the "light line". Of interest on the chart is the minimum value for both $\boldsymbol{\beta}_{\mathbf{0}}$ and $\boldsymbol{\beta}_{\mathbf{c}}$. This is the cut-off frequency for the waveguide containing the slow wave structure capacitive irises. Additionally, $\boldsymbol{\beta}_{\mathbf{c}}$ transitions from a fast wave structure to a slow wave structure and only operates as a slow wave structure over a finite frequency band. When the curve reaches a local maximum, the group velocity goes to zero, per the following relationship.

$$
v_{g}=\frac{\partial \omega}{\partial \beta}
$$

If the group velocity goes to zero, any values beyond this point on the $\boldsymbol{\beta}_{\mathbf{c}}$ curve correspond to evanescent, non-propagating modes. This is almost always the case when a negative group velocity is observed in a transmission line structure [4-1 \& 4-2]. 


\subsection{Slow Wave Structures and Waveguide Slot Arrays}

The usefulness of slow wave structures in this work extends so far as the slow wave structure may be designed to produce a desired $\boldsymbol{\beta}_{\mathbf{c}}$ and a good return loss i.e., when it acts as a well-matched, passive phase shifter. It is anticipated that for a linear array of spacing $\mathbf{s}$, the following $\boldsymbol{\beta}_{\mathbf{c}}$ must be achievable to ensure the slow wave structure will provide the necessary inter-element phase shift for broadside radiation. (This equation will be modified once the phase response of the X-slot is considered, but it will suffice for the discussion at hand.)

$$
\beta_{c}=\frac{2 \pi}{s}
$$

As the first step on a path toward developing a design methodology for passive slow wave structure phase shifters like those seen in Figure 29(d), a review of the classical slow wave structure design procedure is necessary. As will be seen, these classic equations will ultimately provide a useful starting point in the design procedure for a discrete slow wave structure.

\subsection{Slow Wave Structure Analysis}

Slow wave structures first appear in the open literature in 1954 in a paper by R.S. Elliott [2-5]. A thorough development of slow wave structures generated via capacitive iris loading of a waveguide may be found in R.E. Collin's book, Field Theory of Guided Waves [4-3]. The analysis summary below largely follows that work.

The classic waveguide slow wave structure is an infinitely periodic array capacitive irises. A notional cross section is depicted in Figure 35. The dashed lines call out the unit cell of the periodic structure, which has a length, $\mathbf{L}$. In this image, the capacitive structures are irises extending from the bottom wall of the waveguide a distance d, Figure 36. The following development treats the iris as a 
simple equivalent shunt susceptance Figure 37. Alternative shunt capacitive structures will provide identical results, assuming the equivalent susceptance is unchanged.

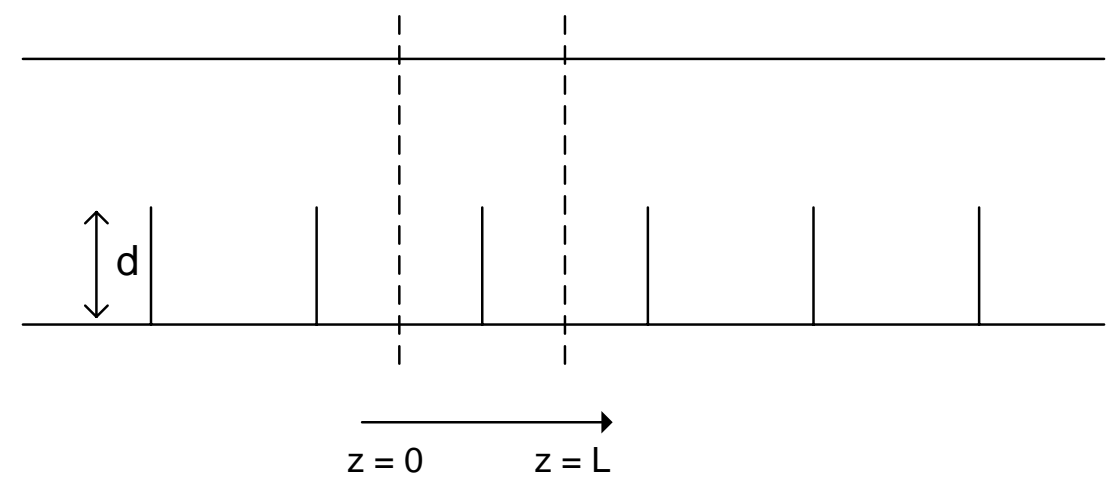

Figure 35 : Capacitive Iris Slow Wave Structure

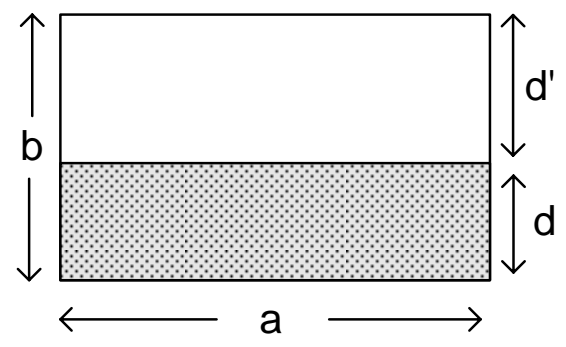

Figure 36 : WG Capacitive Iris

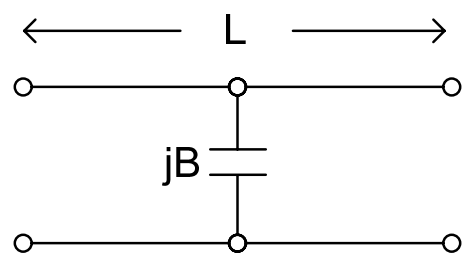

Figure 37 : WG Capacitive Iris Equiv Circuit

The input impedance, $\mathbf{Z}_{\mathbf{i n}}$, seen at the terminals of the unit cell of Figure 37 , when it is terminated in a matched load, $\mathbf{Z}_{\mathbf{L}}$, may be determined as follows, where $\mathbf{B}$ is the equivalent shunt susceptance of the iris and $\boldsymbol{\beta}_{\mathbf{c o}}$ is the cut-off wave number. 


$$
\begin{aligned}
& Z_{\text {in }}=\frac{\left(2 t-B t^{2}\right) /(B+2 t)+j Z_{L}\left(B t+t^{2}-1\right) /(B+2 t)}{j\left(B t+t^{2}-1\right) /(B+2 t)+Z_{L}} \\
& t=\tan \left(\frac{\beta_{0} L}{2}\right) \\
& \beta_{0}=\sqrt{k_{0}^{2}-\beta_{c o}^{2}}
\end{aligned}
$$

The Z-parameters for the capacitive iris unit cell terminated in a matched load may be extracted by comparison with an arbitrary T-network (Figure 38).

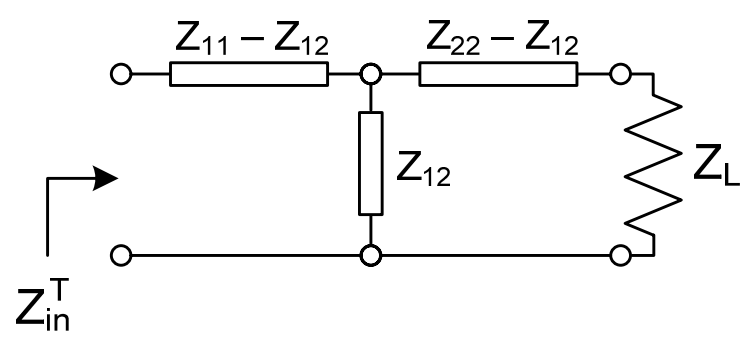

Figure 38 : Arbitary Terminated T-network

$$
Z_{i n}^{T}=\frac{Z_{11}^{2}-Z_{12}^{2}+Z_{11} Z_{L}}{Z_{11}+Z_{L}}
$$

Through a simple comparison of (23) with (20) and a few algebraic manipulations, the following Z-parameters may be extracted for the unit cell capacitive iris.

$$
\begin{aligned}
& Z_{11}^{C}=j \frac{t^{2}+B t-1}{B+2 t} \\
& Z_{12}^{C}=-j \frac{t^{2}+1}{B+2 t}
\end{aligned}
$$


The utility of determining (24) and (25) is apparent when attempting to determine the guided wave number of an infinitely periodic array of the capacitive iris unit cells. The following relationship may be derived for an infinite array of arbitrary, identical T-networks:

$$
\cos \beta_{C} L=\frac{Z_{11}}{Z_{12}}
$$

If the unit cell of the arbitrary T-network is the capacitive iris of Figure 37, a relationship between the slow wave structure guided wave number and simple design variables is obtained after substitution and further algebraic reduction:

$$
\cos \beta_{C} L=\cos \beta_{0} L-\frac{B}{2} \sin \beta_{0} L
$$

\subsection{Capacitive Iris Design}

In (27), a relationship is defined between the guided wave number and three design variables $-\boldsymbol{\beta}_{0}$, the guided wave number; $\mathbf{L}$, the length of the unit cell; and B, the equivalent shunt susceptance of the capacitive iris. The first two variables are trivial, leaving only the calculation of the susceptance to be addressed.

Thankfully, Marcuvitz has already tackled this formidable problem in his classic Waveguide Handbook text, with an approximate expression for infinitely thin capacitive waveguide discontinuities [4-4]. All variables in (28-30) refer to those displayed in Figure 36.

$$
B=\frac{8 b}{\lambda_{g}}\left[\ln (\csc \chi)+\frac{Q \cos ^{4} \chi}{1+Q \sin ^{4} \chi}+\frac{1}{4}\left(\frac{b}{\lambda_{g}}\right)^{2}\left(1-3 \sin ^{2} \chi\right)^{2} \cos ^{4} \chi\right]
$$




$$
\begin{aligned}
& Q=\frac{1}{\sqrt{1-\left(\frac{2 b}{\lambda_{g}}\right)^{2}}}-1 \\
& \chi=\frac{\pi d^{\prime}}{2 b}
\end{aligned}
$$

To determine the relative accuracy of the Marcuvitz expressions, an HFSS simulation was carried out. An iris discontinuity of $6.3 \mathrm{~mm}$ was placed at the center of a length of waveguide with an inner width of $18.3 \mathrm{~mm}$ and a height of $9.15 \mathrm{~mm}$. The wave port phase planes were de-embedded to the plane of the discontinuity. The equivalent shunt susceptance was calculated from the Sparameters and is plotted, alongside the Marcuvitz expression, in Figure 39.

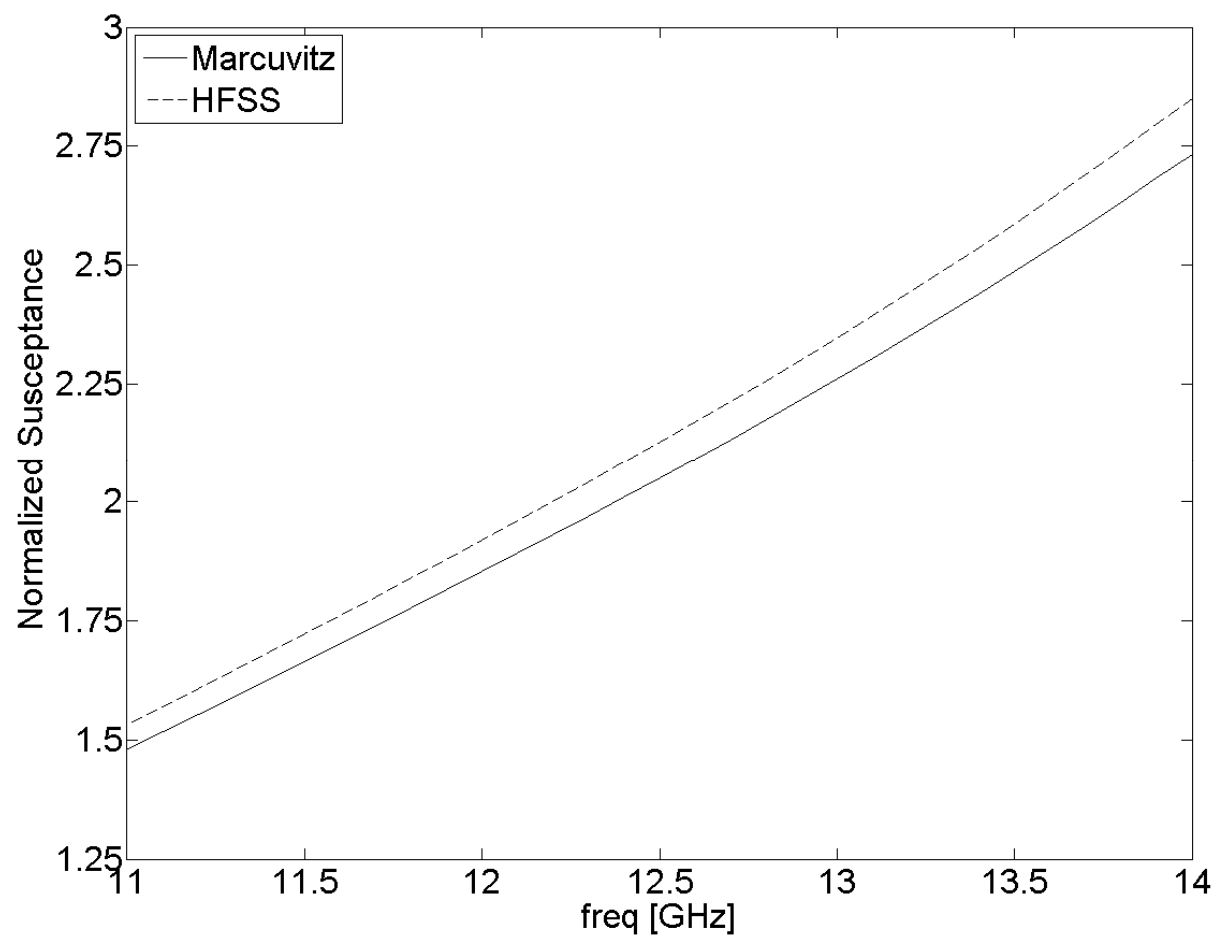

Figure 39 : Theoretical vs. Simulated Iris Susceptance 
Using the simulated data as normative, the Marcuvitz expressions are remarkably accurate. Across the simulated frequency band, an average difference of less than $5 \%$ was observed, providing good confidence in the potential use of the Marcuvitz expression for a slow wave structure theoretical design.

\subsection{Slow Wave Structure Design}

The design equations presented thus far are standard, slow wave structure expressions, given an infinitely periodic structure. Due to the intrinsic difficulties associated with simulating structures with infinite periodicity in the direction of wave propagation, the design equations were not tested beyond what is seen in Figure 39. The ultimate goal of the slow wave structure theoretical development carried out thus far is a reliable design technique for discrete slow wave structures. The next logical step requires a test of the design equations for infinite periodicity in a series of cases with finite periodicity.

A general discrete slow wave structure topology is depicted in Figure 40, where $\mathbf{S}$ is the total length of the device, $\mathbf{d}$ is the height of the irises, $\mathbf{L}$ is the length of the unit cell, and $\mathbf{N}$ is the number of irises. As required in the theoretical analysis, each iris is identical.

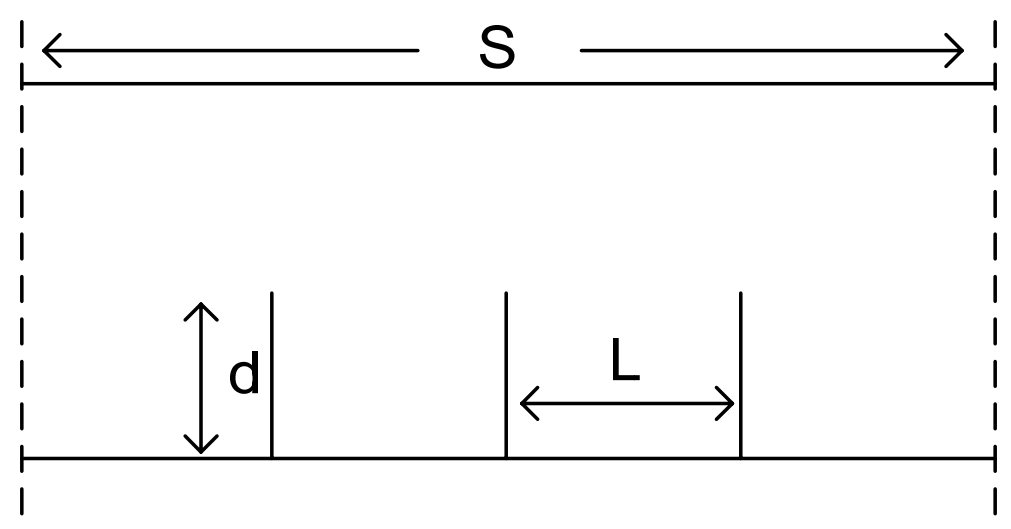

Figure 40: Discrete Slow Wave Structure

For the initial test, $\mathbf{N}=3$ was chosen with $\mathbf{L}=\mathbf{S} / 3$. The value of $\mathbf{S}$ was chosen to be $0.9 \lambda_{0}$ at the design frequency of $12.50 \mathrm{GHz}$, to replicate a required length in an array environment to ensure grating lobe free performance, per (5). 
Additionally, the value of $\boldsymbol{\beta}_{\mathbf{c}}$ was chosen to satisfy the system goals of broadside radiation in a waveguide slot linear array, resulting in a value of $291 \mathrm{rad} / \mathrm{m}$ :

$$
\beta_{C} S=2 \pi
$$

Solving (27), an equivalent susceptance of 1.31 was found. Next, a linesearch optimization in MATLAB [4-5] was performed to solve (28-30) for the height of an iris in a waveguide identical to that employed in the calculation of Figure 39. A value of $5.29 \mathrm{~mm}$ was obtained. To test these design values determined from the Collin and Marcuvitz expressions, another HFSS simulation was performed. The results of which are depicted in Figure 41.

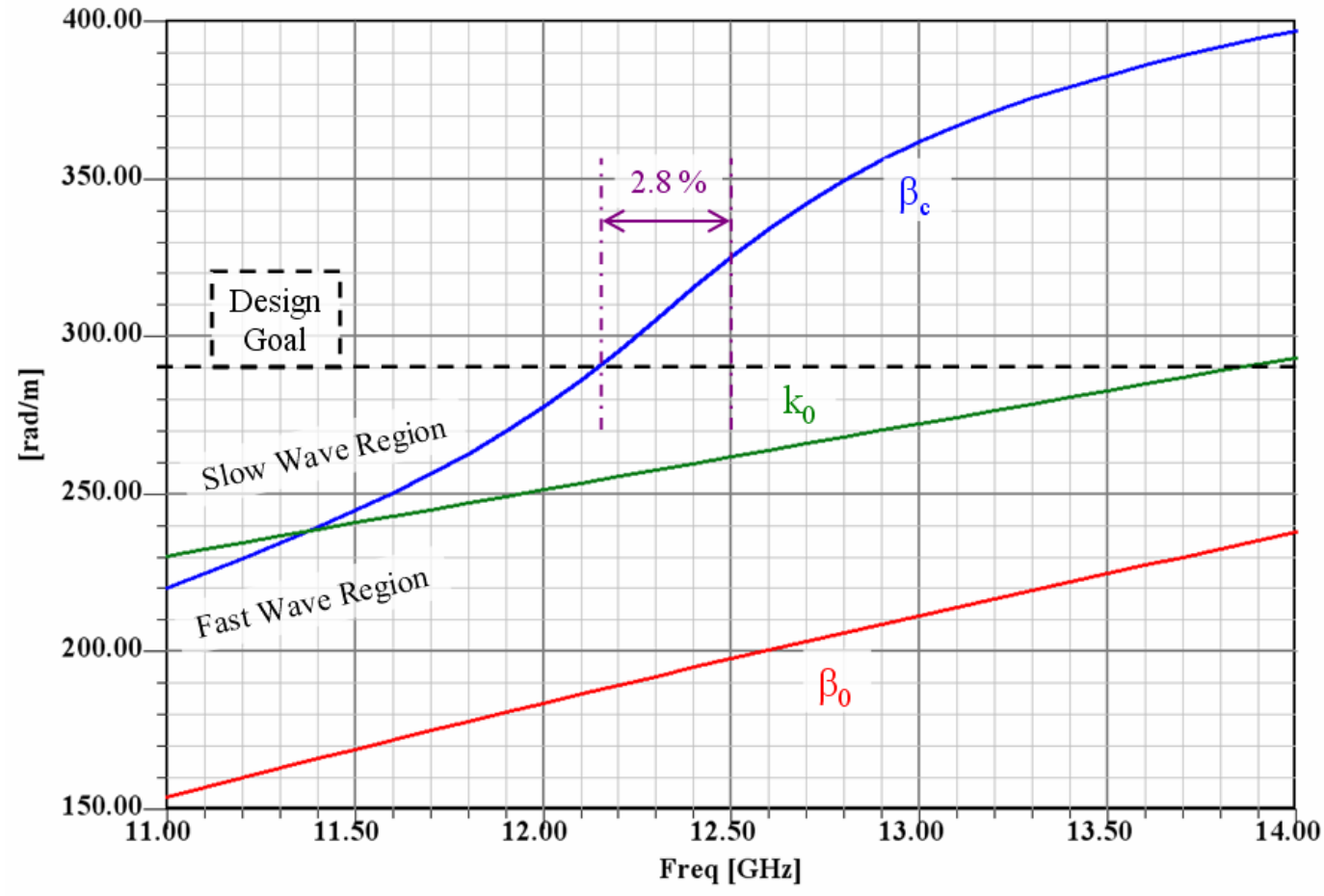

Figure $41: \mathbf{N}=3$ SWS from Theoretical Expressions

As evidenced in the figure, the design equations provided reasonable accuracy for a first pass. When considered within the design environment, however, the $2.8 \%$ shift away from the design goal will translate to an interelement phase shift of $43^{\circ}$ and a beam squint of $7.5^{\circ}$. Given the results of Figure 39, this error was anticipated. What was not clear, however, was the relative 
accuracy of (27) and its applicability to finite slow wave structures. To determine this, a study was conducted where the height of the irises in the initial test case were varied in roughly $0.1 \mathrm{~mm}$ steps until the desired $\boldsymbol{\beta}_{\mathbf{c}}$ was obtained. The results of this study are captured in Table 1.

\begin{tabular}{|c|c|c|c|c|c|c|}
\hline $\mathrm{d}(\mathrm{mm})$ & $\mathrm{B}_{\text {Marcuvitz }}$ & $\mathrm{B}_{\text {HFSS }}$ & $\% \Delta \mathrm{B}$ & $\beta_{\text {Collin }}\left(\mathrm{m}^{-1}\right)$ & $\beta_{\text {HFSS }}\left(\mathrm{m}^{-1}\right)$ & $\% \Delta \beta$ \\
\hline 5.29 & 1.31 & 1.37 & 4.3 & 291.1 & 325.2 & 10.5 \\
\hline 5.20 & 1.26 & 1.32 & 4.5 & 286.8 & 316.5 & 9.4 \\
\hline 5.10 & 1.20 & 1.26 & 4.8 & 282.3 & 310.0 & 8.9 \\
\hline 5.0 & 1.14 & 1.21 & 5.8 & 278.0 & 299.2 & 7.1 \\
\hline 4.9 & 1.09 & 1.13 & 3.5 & 273.9 & 291.5 & 6.0 \\
\hline
\end{tabular}

Table $1:$ N = 3 SWS Error Study

The relative accuracies of both the Marcuvitz and Collin expressions were recorded at each step in iris height. As before, the HFSS results were taken as normative. The iris susceptance error trend is difficult to assess due to the final data point not following the trend of its predecessors. The error trend for the wave number implies the derivative of (27) with respect to $\mathbf{d}$ is less than that of the real device. Due to the complicated structure of (28-30), it is difficult to tell if this is a result of the Marcuvitz expressions or a shortcoming of the Collin equation for the $\mathbf{N}=3$ case. A meaningful point to take from Table 1, however, is the difference in required susceptances to achieve the desired $\boldsymbol{\beta}_{\mathbf{c}}$. The Collin expression requires a $\mathbf{B}$ of 1.31, while the final HFSS value was 1.13. This discrepancy would seem to indicate the Collin expression is inadequate for a small number of irises in the discrete phase shifter.

An assumption was made that as the number of irises increased in the discrete slow wave structure, the accuracy of the Collin expression should 
improve. Thus, if the error in the Marcuvitz expression could be accounted for, the theoretical design approach should achieve acceptable levels of accuracy. A similar study to that done for $\mathbf{N}=3$ was carried out up to $\mathbf{N}=10$, the results of which are captured in Table 2.

\begin{tabular}{|c|c|c|c|}
\hline $\mathrm{N}$ & $\mathrm{B}_{\text {collin }}$ & $\mathrm{B}_{\text {hfss }}$ & $\% \Delta$ \\
\hline 3 & 1.31 & 1.13 & 16.0 \\
\hline 4 & 1.10 & 0.93 & 18.3 \\
\hline 5 & 0.92 & 0.82 & 12.2 \\
\hline 6 & 0.79 & 0.75 & 5.3 \\
\hline 7 & 0.69 & 0.67 & 3.0 \\
\hline 8 & 0.60 & 0.63 & -4.8 \\
\hline 9 & 0.54 & 0.59 & -8.5 \\
\hline 10 & 0.49 & 0.55 & -10.9 \\
\hline
\end{tabular}

Table 2 : SWS B Study - HFSS vs. Collin

The anticipated relationship was confirmed by simulation with an improvement in the Collin error observed with increasing $\mathbf{N}$. If the Collin expression were complete, the error relationship would be asymptotic. However, negative error values are calculated above $\mathbf{N}=7$ - i.e., where Collin predicts a smaller susceptance than that required. This error may be understood as follows. Only the dominant waveguide mode is considered in the transmission line analysis development of the slow wave structures, resulting in the design equation (27). Given a single-mode incident field, the iris obstructions will scatter energy into a spectrum of higher-order-modes, all but one of which will be evanescent. This evanescent, non-propagating energy may be understood as the cause of the equivalent reactance. When the spacing, $\mathbf{L}$, decreases to the point that a nontrivial amount of evanescent energy begins to contribute to the field coupling between irises, then a single-mode transmission line analysis will be insufficient. 
For the purpose of this work and its regular usage of the $\mathbf{N}=3$ discrete slow wave structure, this phenomena, while acknowledged, will be ignored.

Thus the Collin and Marcuvitz expressions have empirically proven themselves to be reliable starting points for a discrete slow wave structure design. Achieving a design that meets the required wave number is a fairly simple process which could be carried out with a commercial software package and an optimization algorithm. The issue of completing a discrete slow wave structure design which meets both the wave number and return loss requirements for a traveling wave dual CP slot array, discussed below, will further emphasize the need for and utility of commercial software with optimization packages.

\subsection{Unoptimized Slow Wave Structure Return Loss}

To function well within the traveling wave design procedure outlined in Chapter 3, the slow wave structure passive phase shifters must be well-matched to the waveguide. For the purpose of this work, it is assumed the $S_{11}$ value must be below $0.1(-20 \mathrm{~dB})$ to be considered adequate.

To begin an investigation of the return loss characteristics of discrete slow wave structures, the HFSS return loss data for the configurations of Table 2 were plotted in Figure 42. The calculated bandwidths for each curve are captured in Table 3.

As seen in the plot, each iteration of $\mathbf{N}$ produced a return loss of better than $-20 \mathrm{~dB}$ at some point within the simulated frequency band. Additionally, the bandwidth changed very little for $\mathbf{N}>3$. The problem, from the perspective of developing a design approach for discrete slow wave structures, was that the wellmatched frequency range shifted with increasing $\mathbf{N}$ and did not generally correlate to the design frequency-12.5 GHz here-where $\boldsymbol{\beta}_{\mathbf{c}}$ was the correct value to produce broadside radiation. 


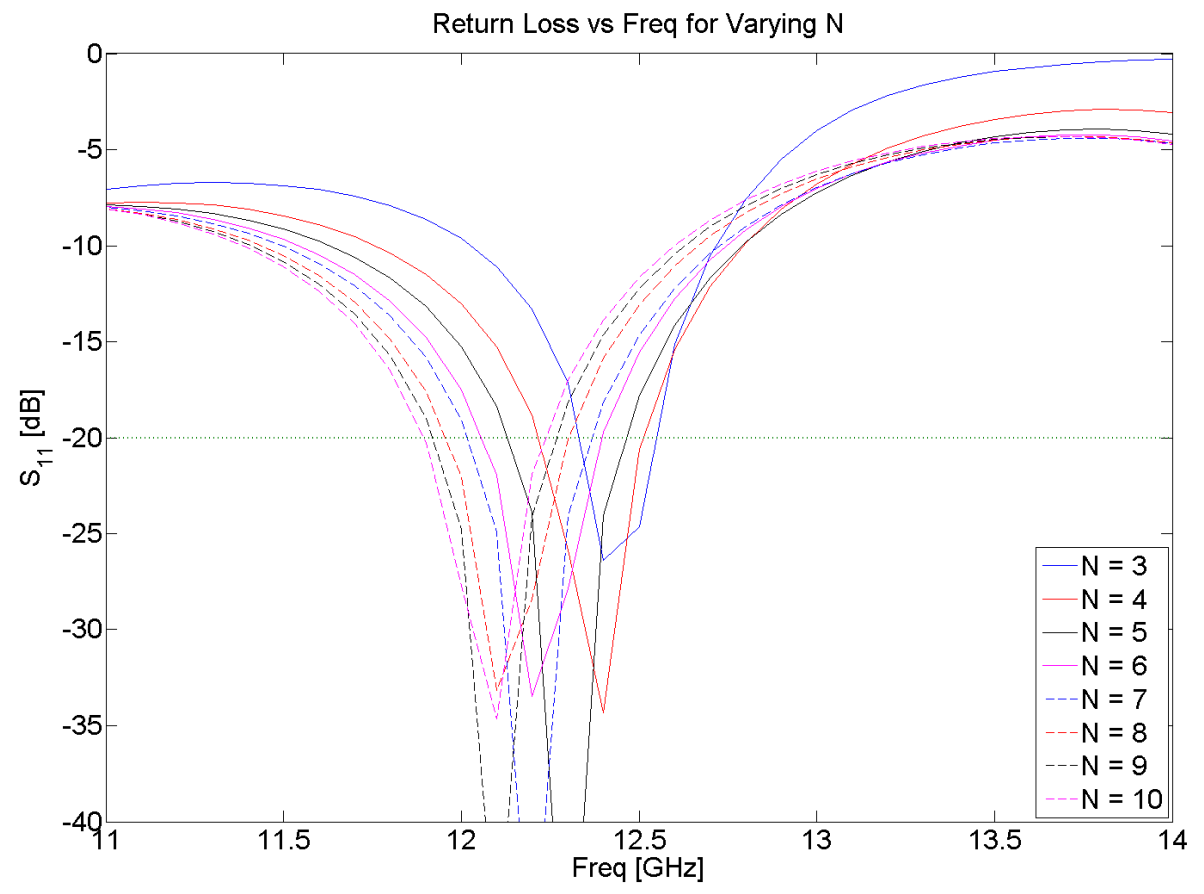

Figure 42 : Unoptimized SWS Return Loss

\begin{tabular}{|c|c|}
\hline $\mathrm{N}$ & \% BW \\
\hline 3 & 1.6 \\
\hline 4 & 2.4 \\
\hline 5 & 2.6 \\
\hline 6 & 2.9 \\
\hline 7 & 2.9 \\
\hline 8 & 2.9 \\
\hline 9 & 2.9 \\
\hline 10 & 2.7 \\
\hline
\end{tabular}

Table 3 : Unoptimized Discrete SWS BW 


\subsection{Optimizing Slow Wave Structure Return Loss}

To improve the discrete slow wave structure return loss while maintaining the wave number performance, an optimization process was employed. This is technique is commonly employed when designing complicated electromagnetic structures which are intractable via theoretical analysis. It could be argued that the majority of modern RF engineering relies more heavily on optimization algorithms driven by simulation tools than traditional theoretical analysis.

To efficiently optimize the slow wave structure, a new piece of software was employed. The simulations to this point had been limited entirely to HFSS, a generic, robust FEM commercial package which is capable of simulating slow wave structures, but does not do so efficiently. WASP-NET, a commercial code designed specifically for waveguide structures, was utilized to perform the optimization of the slow wave structures [4-6]. It uses the Mode-Matching technique to simulate the effects of waveguide discontinuities highly efficiently and transmission line theory to transform these effects along the intervening space between discontinuities.

To demonstrate the efficiency of this technique, the $\mathbf{N}=3$ structure from above was simulated in WASP-NET. The solution time required was approximately $1 \mathrm{sec}$ per frequency point. By contrast, the HFSS solution required over 5 minutes for the first frequency point and approximately another 5 minutes to complete the interpolating sweep across the frequency band. The difference between $1 \mathrm{sec}$ and 10 minutes would be admittedly trivial if only a single design iteration were required. However, when optimization algorithms are involved and several hundred steps may be required in the design space before the goals are achieved, a reduction in cost function evaluation time by two orders of magnitude is extremely important.

\subsubsection{Optimized Slow Wave Structure Return Loss}

In order to optimize the return loss and maintain the desired $\boldsymbol{\beta}_{\mathbf{c}}$, it was necessary to increase the degrees of freedom in the design space. In the first attempted 
optimization configuration, the iris spacing was held constant, while different iris heights were permitted. It was conjectured that the discrete slow wave structure would have improved return loss performance if the loading presented to the waveguide from each successive iris were tapered. To ensure the effect would be identical for a wave propagating from either direction, a symmetric taper was enforced. The updated slow wave structure layout and optimization constraints are depicted below (Figure 43).

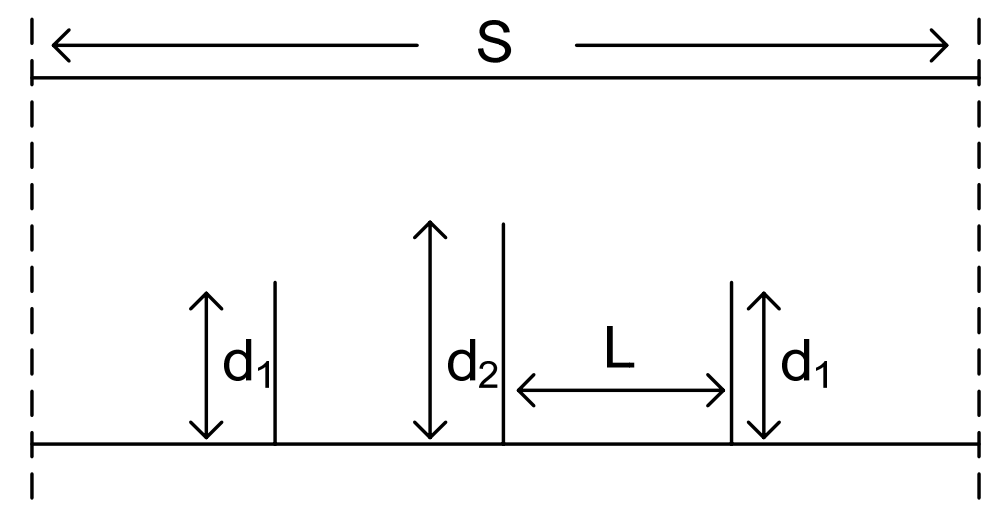

Figure 43 : SWS Configuration for Optimization

$$
\begin{aligned}
& d_{n} \begin{cases}<d_{n+1} & n<N / 2 \\
=d_{n+1} & n=N / 2 \\
>d_{n+1} & n>N / 2\end{cases} \\
& L=S / N
\end{aligned}
$$

WASP-NET includes several optimization algorithms, including random walk, genetic algorithms, a proprietary technique termed "Extreme", and an approach employing Powell's direct search method [4-7 and 4-8]. To maintain the philosophical approach of developing a widely accessible design approach, the Powell's method was chosen. This method is more efficient than a random walk and is considered more likely to be repeatable by someone using an external optimizer than the genetic algorithm because WASP-NET does not disclose the genetic coding approach it uses. Obviously the proprietary "Extreme" technique would also only be available to anyone using WASP-NET, rather than any of the 
other commercially available software packages designed specifically for waveguide analysis. Thus, the decision to employ the Powell's method algorithm was made from the perspective of maintaining a generalized, widely accessible design approach, rather than on purely technical grounds.

The optimization algorithm was allowed to vary the height of each element, $\mathbf{d}_{\mathbf{n}}$, and was constrained by the relationships given above. A starting point was provided based on the structures of Table 3. It was believed that a solution which simultaneously provided the desired $\boldsymbol{\beta}_{\mathbf{c}}$ and good return loss would be close to the original design with the correct $\boldsymbol{\beta}_{\mathbf{c}}$. The optimization goals required $S_{11}$ be less than 0.1 at three frequency points $(12.2,12.45$, and $12.7 \mathrm{GHz})$ and $\boldsymbol{\beta}_{\mathbf{c}}$ to remain equal to 291.1 at $12.5 \mathrm{GHz}$.

For each value of $\mathrm{N}$, the iteration required around 100 steps and 3 minutes to converge. The optimized return loss and bandwidths are captured in Figure 44 and Table 4, respectively.

It was observed that after the initial optimization, device bandwidth increased by $50 \%$ to $80 \%$ for each value of $\mathbf{N}$. Additionally, the design goal of $-20 \mathrm{~dB}$ return loss was met simultaneously, for each value of $\mathbf{N}$, at $12.2 \mathrm{GHz}$. While these two improvements were considered positive progress, they were also deemed insufficient to justify a full design methodology. 


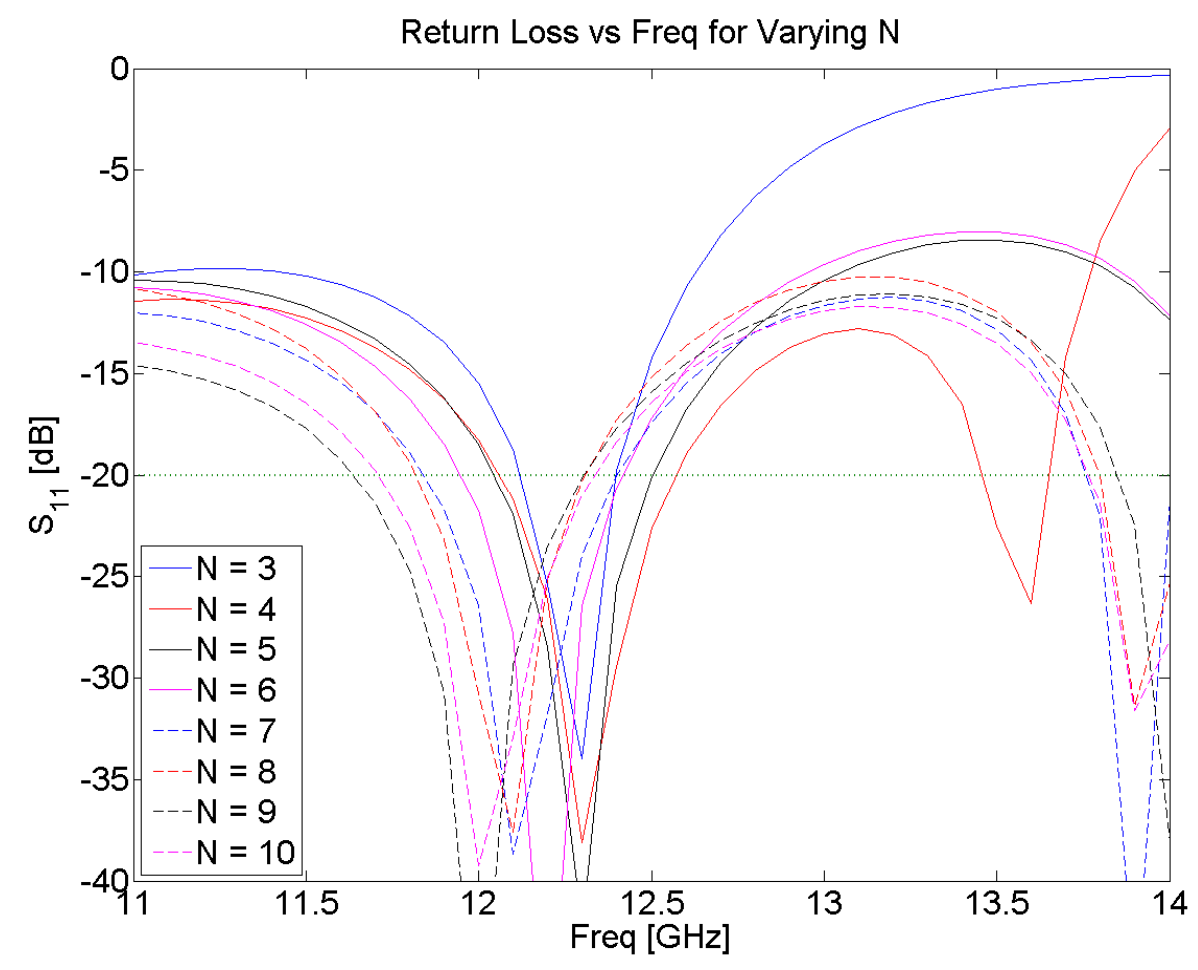

Figure 44 : Optimized SWS Return Loss

\begin{tabular}{|c|c|}
\hline $\mathrm{N}$ & $\% \mathrm{BW}$ \\
\hline 3 & 2.4 \\
\hline 4 & 4.1 \\
\hline 5 & 3.7 \\
\hline 6 & 3.9 \\
\hline 7 & 4.6 \\
\hline 8 & 4.1 \\
\hline 9 & 5.4 \\
\hline 10 & 5.3 \\
\hline
\end{tabular}

Table 4 : Optimized SWS BW 


\subsubsection{Re-Optimized Slow Wave Structure Return Loss}

The next step in developing a discrete slow wave structure design approach required the relaxation of an equality constraint in the optimization process. To this point, it had been assumed that the irises should be distributed equally within the waveguide unit cell length. This assumption was tested by removing the equality constraint on iris spacing from the first set of optimization attempts (33). All other constraints, starting points, and goals were left unchanged and the process was repeated.

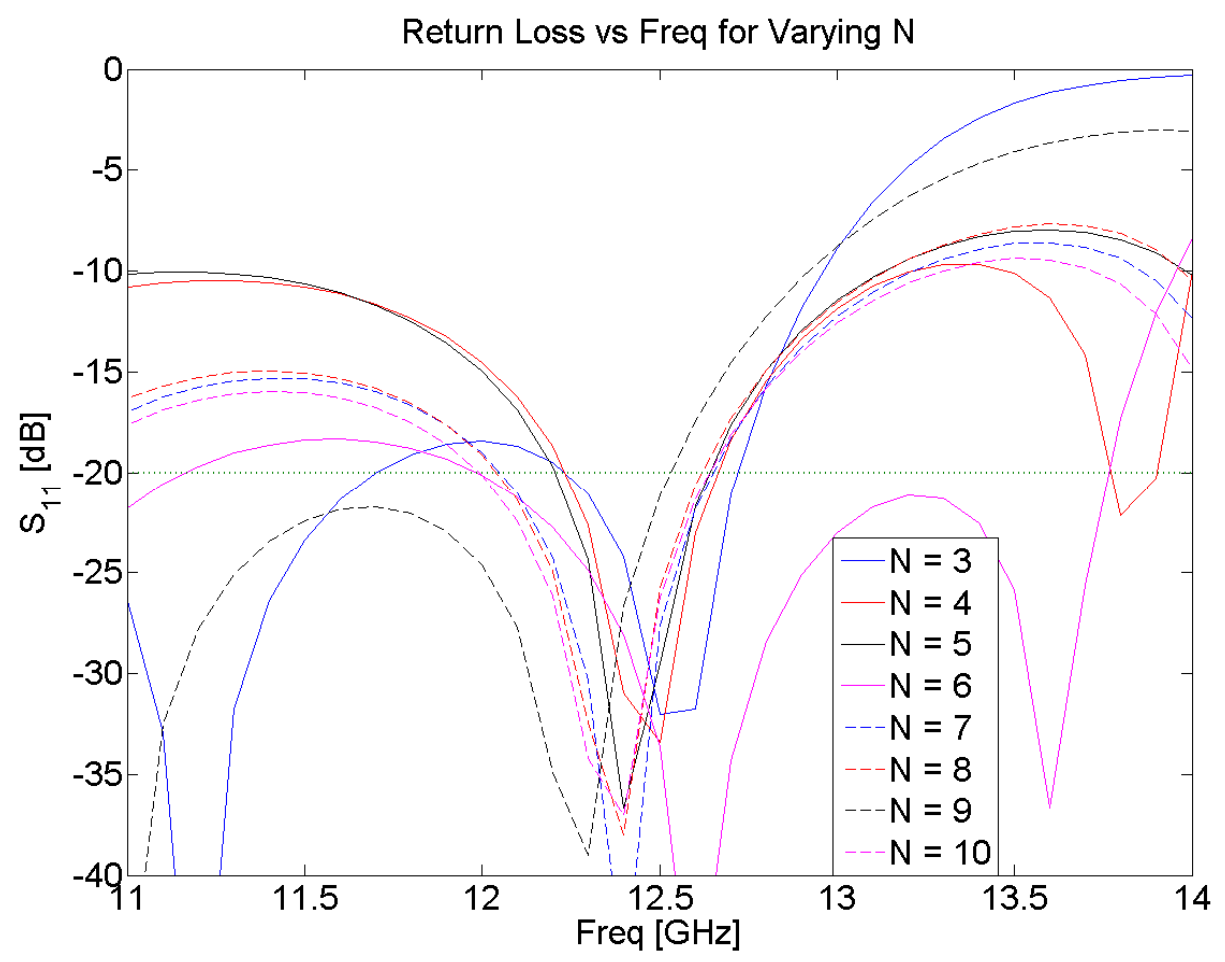

Figure 45 : Re-Optimized SWS Return Loss 


\begin{tabular}{|c|c|}
\hline $\mathrm{N}$ & $\% \mathrm{BW}$ \\
\hline 3 & 3.8 \\
\hline 4 & 3.9 \\
\hline 5 & 3.6 \\
\hline 6 & 14.0 \\
\hline 7 & 4.9 \\
\hline 8 & 4.8 \\
\hline 9 & 17.2 \\
\hline 10 & 5.4 \\
\hline
\end{tabular}

Table 5 : Re-Optimized SWS BW

Two important observations can be made from the second optimization analysis. First, for each value of $\mathbf{N}$, the iteration was able to achieve the return loss goal at $12.45 \mathrm{GHz}$. This would indicate that after relaxing the equality constraint and increasing the degrees of freedom in the design space to include the iris spacing, the optimization technique can simultaneously meet the design goals for $\boldsymbol{\beta}_{\mathbf{c}}$ and return loss. Second, while the bandwidth of most of the iterations improved only marginally over the previous optimization attempt, two of them more than tripled. A close examination of the $\mathbf{N}=6$ and $\mathbf{N}=9$ curves revealed the source of this bandwidth increase. A series of pass bands were generated by each structure. When these bands had an optimal return loss and occurred close enough in frequency to overlap, a composite, wider band response was generated.

The relative irrelevance of the large operating bandwidths can be determined with a quick glance at Figure 41. In order to maintain a broadside beam across the entire useable return loss bandwidth of operation, $\boldsymbol{\beta}_{\mathbf{c}}$ must hold a constant value. If this does not occur, an inter-element phase shift will exist and 
cause the beams associated with each sense of $\mathrm{CP}$ to squint in opposite directions away from broadside. However, as indicated in (18), a constant $\boldsymbol{\beta}_{\mathbf{c}}$ across frequency would imply an infinite group velocity. Not wishing to defy Relativity and attempt to induce superluminal propagation, further discussion of increasing the bandwidth of operation within a discrete slow wave structure design approach will not be considered in this work.

\subsection{A Discrete Slow Wave Structure Design Methodology}

Based on the results of the studies presented above, the following discrete slow wave structure design methodology for passive phase shifter operation within linear arrays of dual $\mathrm{CP}$ slot radiators is suggested.

1. Acquire array operational details (frequency, spacing, etc.).

2. Choose an initial $\mathbf{N}$ and calculate the required $\boldsymbol{\beta}_{\mathbf{c}}$.

3. From the Collin expression, calculate the required $\mathbf{B}$.

4. From the Marcuvitz expressions, solve for iris height, $\mathbf{d}$.

5. Simulate the initial structure with equal iris heights and the irises spaced evenly throughout the unit cell. (Software selection and efficiency is irrelevant at this stage.)

6. If the required return loss and $\boldsymbol{\beta}_{\mathbf{c}}$ performance are not met, optimize the structure with a piece of software which can efficiently handle waveguide discontinuities. Use the Collin/Marcuvitz expression as a starting point and permit the optimization algorithm to adjust the height of each iris and the spacing between irises.

7. If the return loss and $\beta_{\mathrm{c}}$ goals are not met simultaneously, increase $\mathrm{N}$ by 1 and repeat steps $2-6$. (An increase in $\mathrm{N}$ will increase the degrees of freedom in the design space, improving the probability of finding a solution.) 
The proposed design methodology was tested repeatedly and produced good results. Rarely was a slow wave structure with more than 3 irises required and typically two solutions could be found. One solution required a slight shift in iris spacing and height, but all iris heights were fairly equal. The second solution required a shift in spacing and included the height taper described above. The presence of multiple solutions should be expected - that is, a single, unique solution should not be anticipated with such generic optimization goals. This is not equivalent to stating that a solution is easily found and, thus, does not devalue the method of choosing the Collin/Marcuvitz solution as a reliable, robust starting point for the optimization process. Finally, it is believed that as $\mathbf{N}$ increases, the number of solutions will generally also increase, but this theory has not been extensively tested. 


\section{Chapter 5: X-Slot Parametric Study and Design Rules}

The proposed array topology includes both a passive slow wave structure phase shifter, to ensure broadside radiation, and an $\mathrm{X}$-slot radiating element, to ensure dual-CP operation. Having tackled the problem of developing a design procedure for the passive phase shifters, the next task was to develop design guidelines for the $\mathrm{X}$-slot radiator.

A theoretical point of departure existed for an X-slot design procedure investigation, much like the slow wave structure development above. As will be seen below, however, a set of design guidelines were developed for the X-slot radiator, instead of the step-by-step design methodology seen in Chapter 4. X-slot performance parameters were observed to be sensitive enough to shifts in design variables to justify this approach. That is, it was discovered that a unit cell X-slot analysis with application-specific details will be required for each unique array design process and developing traditional tools like slot conductance curves would either be too complicated to be useful or too simple and thus potentially misleading.

\subsection{A Discrete Slow Wave Structure Design Methodology}

As discussed above, Simmons' original paper featuring the $\mathrm{X}$-slot radiator included a single design equation. This was presented in Chapter 3 , along with a plot of the normalized transverse and longitudinal magnetic field magnitudes for a $\mathrm{TE}_{10}$ mode in a rectangular waveguide (Figure 22). As discussed previously, the operational principle of an X-slot radiator, as proposed by Simmons, is that two points exist within any transverse cross-section of a rectangular waveguide where the magnetic fields and, by extension, surface currents, have equal magnitude, are orthogonal, and are in phase quadrature. Because these are the three required conditions for generating a $\mathrm{CP}$ field, then an aperture at this point should produce good CP radiation. This theoretical basis of operation is captured well in the 
single design equation (10) he provided, however, it does little to address the problem confronted here, which is how to design an X-slot radiator for array applications.

As stated in the proposed design procedure at the end of Chapter 3, the radiated power, radiated axial ratio, forward scattered phase, and return loss are four critical design parameters, which must be accounted for in the design procedure of the element in order for the array to function properly. Since none of those parameters are addressed in either Simmons' equation or his theory of operation, a parametric study was performed. The X-slot design variables addressed are seen in Figure 46, where $\mathbf{L}_{1}$ and $\mathbf{L}_{2}$ refer to the lengths of each slot, $\xi$ is angle between the two slots, $\psi$ is the rotation angle of the slot relative to the waveguide walls, and $\boldsymbol{\delta}$ is the slot offset. Two additional variables, not shown, were also studied $-\mathbf{t}$, waveguide wall thickness, and $\mathbf{w}$, slot width.

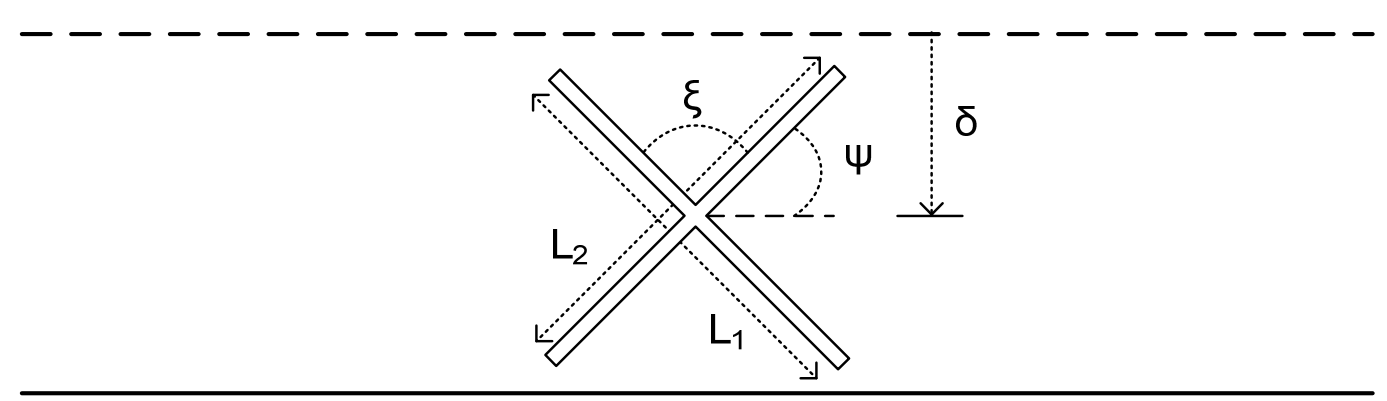

Figure 46 : X-Slot for Parametric Study

What follows is a methodical presentation of the parametric study results. Each design variable was considered independent of all others, thus only a single parameter was changed at a time. With each variable change, the relationships between that variable and return loss, axial ratio, forward scattered phase, and radiated power were considered. Plots of slot performance against shifts in each 
design variable were generated and analyzed, resulting in a set of X-slot design guidelines for traveling wave, dual-CP arrays.

To perform the parametric study, unit cell simulations were performed in HFSS. Each slot was fed from an identical waveguide, was permitted to radiate into an infinite half-space, and the calibrated phase plane of each feed port was de-embedded to the center of the X-slot. Thus, the calculated phase of $\mathrm{S}_{21}$ was the forward scattered phase perturbation the X-slot applies to the source field. Finally, X-slots with zero thickness were used to carry out the bulk of the simulations. The role of slot thickness was investigated separately.

\subsection{X-Slot Parametric Study $: \delta$}

Since Simmons' design equation only addresses the X-slot offset, $\boldsymbol{\delta}$, it was decided this would be an appropriate place to begin the parametric study. After determining a waveguide size $(20 \mathrm{~mm} \times 10 \mathrm{~mm})$ and design frequency $(12.5$ $\mathrm{GHz}$ ), Simmons' equation was used to calculate a required slot offset of $6.86 \mathrm{~mm}$. The inner slot angle was $90^{\circ}$ and the slot rotation angle was $45^{\circ}$. The longest slot length that would fit entirely within the face of the waveguide wall was chosen $(8.0 \mathrm{~mm})$. Three variations of slot offset were tested $-6.0 \mathrm{~mm}, 6.5 \mathrm{~mm}$, and 6.86 $\mathrm{mm}$.

The radiation performance is captured in Figure 47 below. Note, Copolarized gain is plotted in Red, cross-polarized gain is plotted in Blue, and Axial Ratio is plotted in Green. These conventions will be maintained throughout the rest of the parametric study for every radiation pattern. The S-parameter performance is captured in Figure 48 where the magnitude of $S_{21}$ is seen in Blue, the magnitude of $\mathrm{S}_{11}$ is seen in Red, and the phase of $\mathrm{S}_{21}$ is seen in Green. Again, these color conventions will be employed throughout the remainder of this chapter for every plot of S-parameter performance against a design variable.

The validity of Simmons' design equation was seen in the radiation pattern data. As the slot offset approached the calculated value of $6.86 \mathrm{~mm}$, the axial ratio performance improved. The S-parameter data, however, demonstrated 
the impracticality of Simmons' equation. To fit an X-slot entirely within the face of a rectangular waveguide and center it at the calculated offset, the slot lengths will be too short to couple any appreciable amount of energy. In this example, the slot is around one-third of a wavelength.

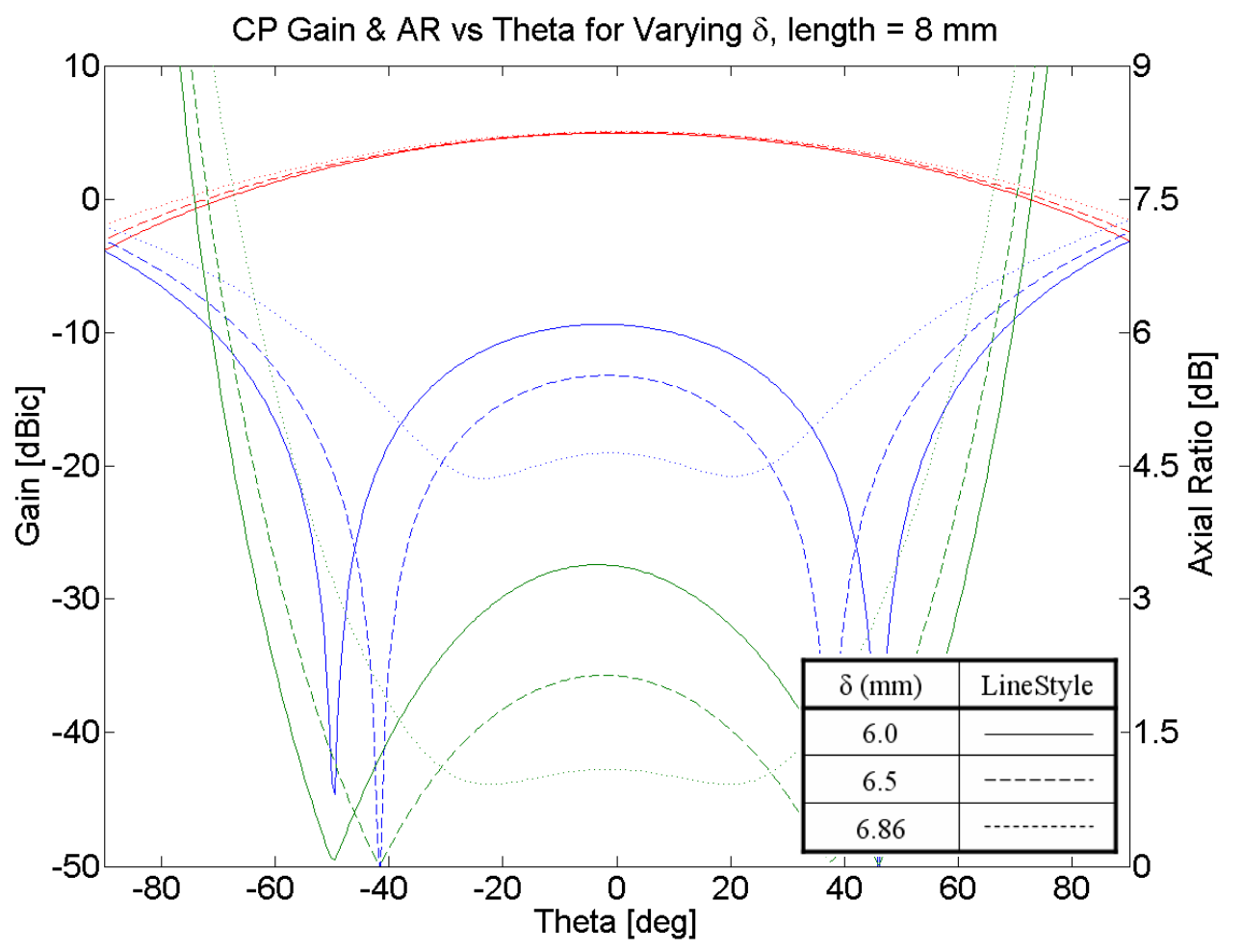

Figure 47 : 8 mm X-Slot, 12.5 GHz Radiation Performance vs. $\delta$ 


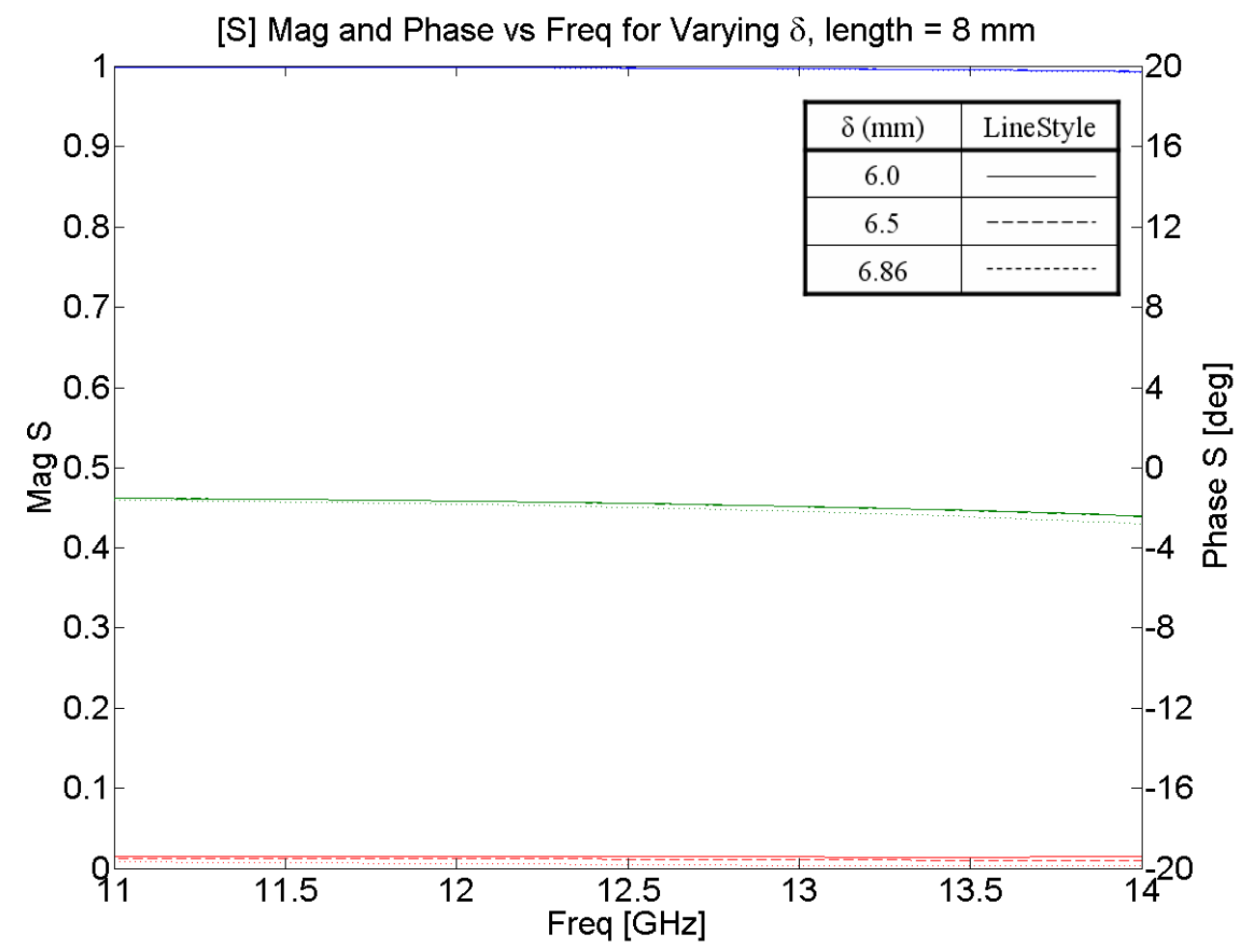

Figure $48: 8$ mm X-Slot, [S] vs. $\delta$

Typically, waveguide slots exhibit some form of a resonance when they are around a half-wavelength long. Thus, to improve the relevance of the test, a slot with an $11.5 \mathrm{~mm}$ length was next tested. Due to the increased slot length, the range of achievable slot offset values was restricted to below $5.70 \mathrm{~mm}$. Four values were tested at and below this offset value. The radiation performance and S-parameter results of the simulation are captured in Figure 49 and Figure 50.

As anticipated by Simmons' equation, the axial ratio performance improved with increasing offset, but was generally quite good over all the values tested. A slight change in the S-parameter performance was observed with variations in slot offset. As slot offset increased, the return loss generally also improved, perhaps indicating a relationship between return loss and axial ratio. The resonant frequency shifted with slot offset. This result was anticipated as a similar effect is observed with linearly polarized, longitudinal slots [3-10]. As the slot offset increases, the aperture field is perturbed by the waveguide side wall. 
This interaction causes a shift in the slot reactance, which, in turn, shifts the resonant frequency.

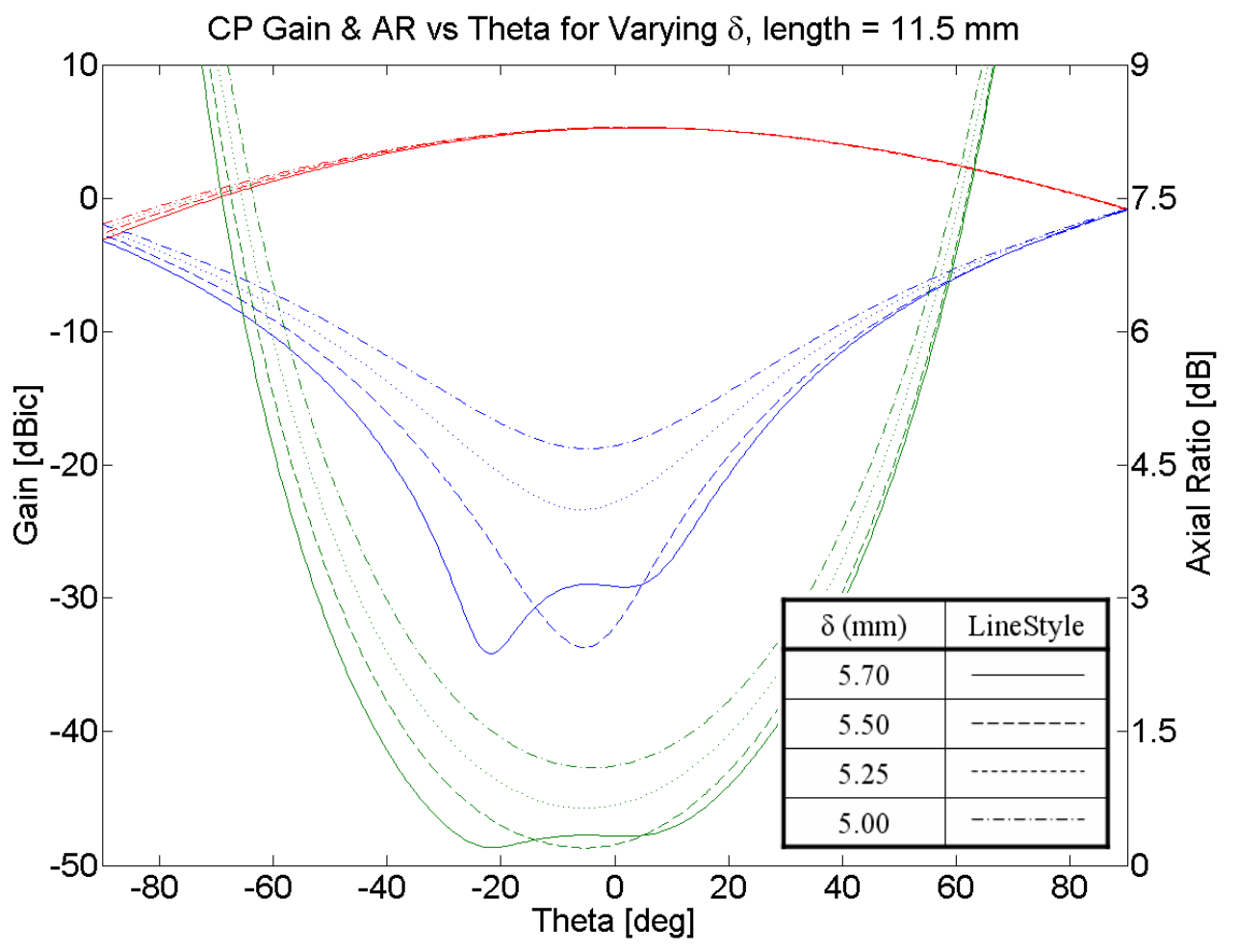

Figure 49 : 11.5 mm X-Slot, 12.5 GHz Radiation Performance vs. $\delta$ 


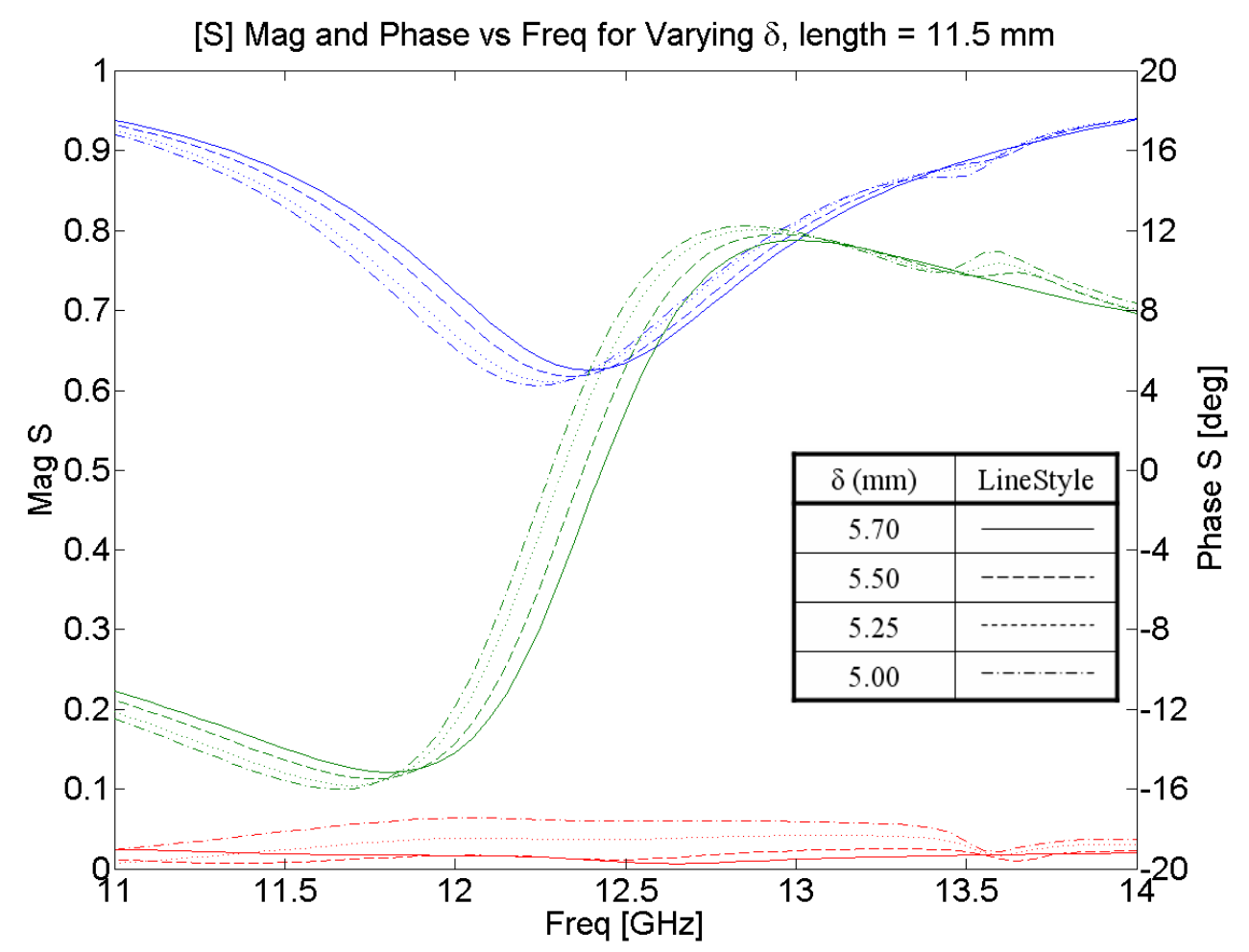

Figure 50 : 11.5 mm X-Slot, [S] vs. $\delta$

Aside from the previously mentioned slight relationship between $\mathrm{S}_{11}$ and slot offset, the small magnitude of the return loss was of particular interest. At resonance, where the $\mathrm{X}$-slot was radiating the maximum amount of power, the slot impedance is matched remarkably well to the waveguide feed line. This was an unexpected result that, as will be seen, makes the $\mathrm{X}$-slot a particularly good choice for the proposed traveling wave array topology.

To further test the relationship between slot offset and performance, a more extensive series of simulations were carried out where the slot offset was varied from $0 \mathrm{~mm}$ to the terminal value of $5.70 \mathrm{~mm}$. The minimum axial ratio and maximum return loss are plotted against the slot offset in Figure 51 and Figure 52, respectively. 


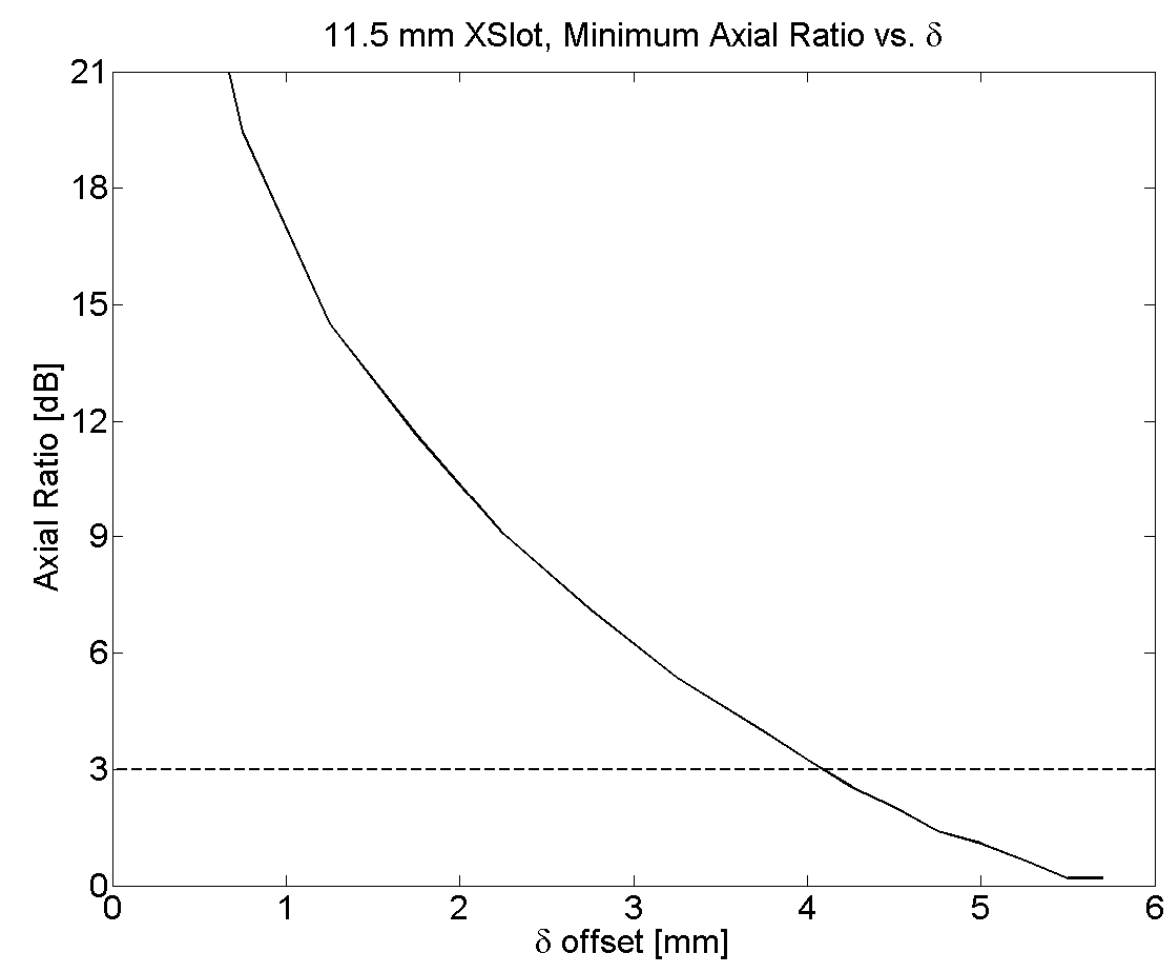

Figure $51: 11.5$ mm X-Slot, 12.5 GHz AR vs. $\delta$

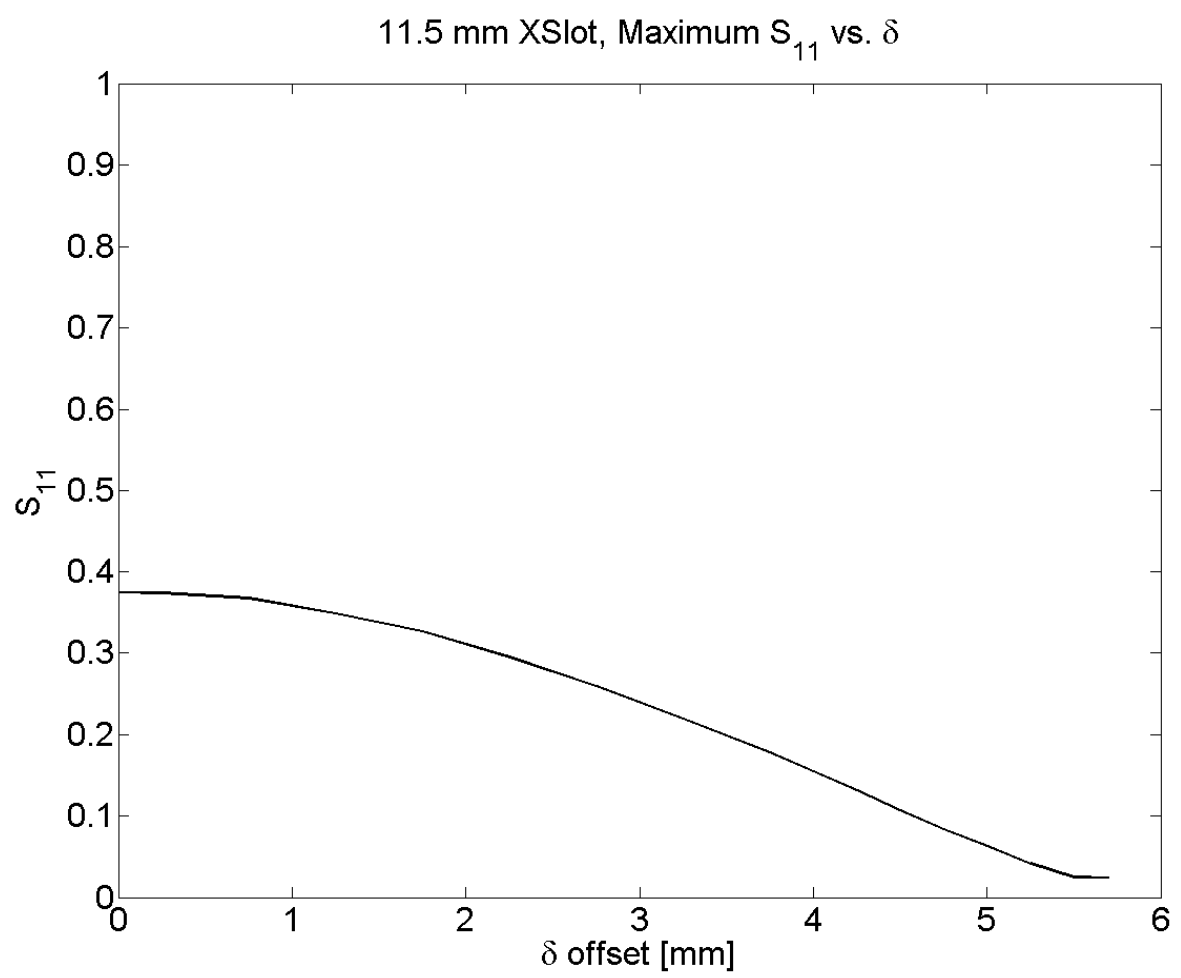

Figure 52 : $11.5 \mathrm{~mm} X$-Slot, $\operatorname{Max} S_{11}$ vs. $\delta$ 
As seen in the earlier results, both axial ratio and return loss improved with increasing slot offset. Other parameters, including the maximum radiated power, the position of the minimum axial ratio, and the resonant frequency were also tested against variation in slot offset. Aside from the previously mentioned shift in resonant frequency, none of the other relevant X-slot performance metrics displayed a relationship to slot offset worth noting.

In conclusion of the slot offset study, simulation data confirmed Simmons' equation. By itself, the equation is of little use in the design of a practical X-slot, due to the length restrictions it places on the slot. Basic trends predicted by the equation were confirmed as X-slot axial ratio was seen to improve with increasing slot offset. Unexpectedly, the return loss was also observed to improve with increasing slot offset and reached remarkably low levels (less than $-30 \mathrm{~dB}$ ) for a slot offset as far as possible.

\subsection{X-Slot Parametric Study : $\xi$}

The next slot design variable studied parametrically was the slot inner angle, $\xi$. Because the polarization of each leg of the X-slot is highly linear and one of the primary requirements for radiating a circularly polarized field is to have two orthogonal components, it was believed that the primary relationship between slot inner angle and slot performance would be observed in the axial ratio. Given the potential relationship observed between return loss and axial ratio in the first parametric study, however, it was also anticipated that a relationship between return loss and slot inner angle might be observed.

To permit a range of slot inner angles such that the slot fit entirely within the waveguide wall, a non-optimal slot offset was chosen at $5.25 \mathrm{~mm}$. After performing a set of parametric simulations where the slot angle varied from $80^{\circ}$ to $100^{\circ}$, the following radiation and S-parameter charts were generated. 


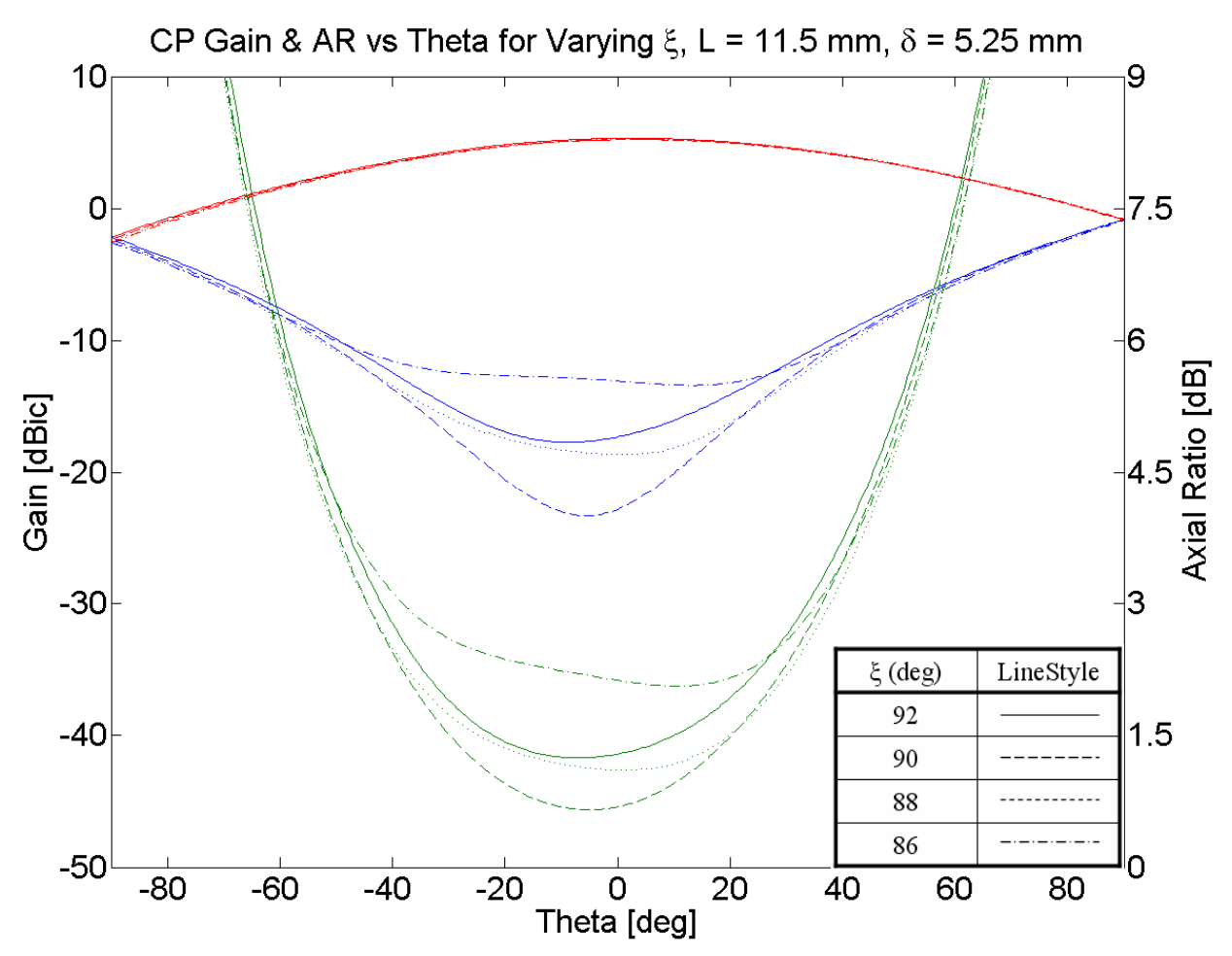

Figure 53 : 11.5 mm X-Slot, 12.5 GHz Radiation Performance vs. $\xi$

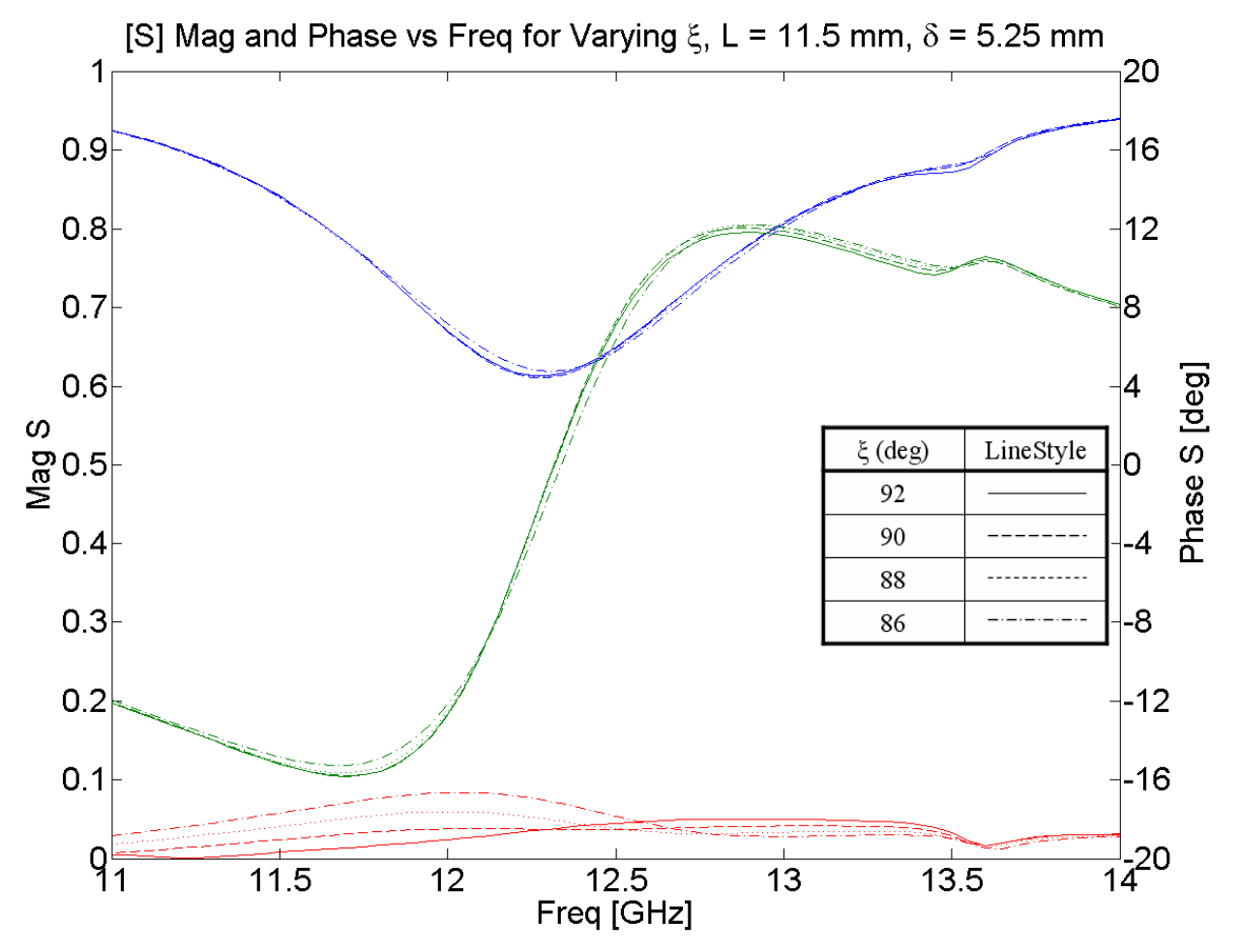

Figure $54: 11.5 \mathrm{~mm} \mathrm{X-Slot,} \mathrm{[S]} \mathrm{vs.} \xi$ 
As expected, the axial ratio was observed to shift with slot inner angle. Interestingly, the only relationship observed with S-parameter performance was the return loss. The effect of the slot inner angle on other performance metrics was studied. Aside from a curious, and rather unexpected, relationship between the spatial position of minimum axial ratio and the slot inner angle, little useful information was gleaned beyond what may be found in Figure 53 and Figure 54.

To summarize the slot inner angle parametric study, the results were largely as anticipated. As the slot inner angle shifts and removes the orthogonality condition, the radiated field axial ratio increases. Additionally, the suspected relationship between return loss and axial ratio was strengthened. For the purposes of a broadside radiating X-slot array, it is recommended that the inner slot angle of each element should be fixed at $90^{\circ}$.

\subsection{X-Slot Parametric Study $: \psi$}

The next variable to be studied parametrically was the slot rotation angle, $\psi$. In the limit of rotation at $0^{\circ}$ and $90^{\circ}$, the X-slot could be considered a T-slot where the longitudinal and transverse components intersect. As seen above, a T-slot is capable of radiating low axial ratio $\mathrm{CP}$ fields. Thus, it was not expected that the slot rotation angle would play a large role in slot performance. On a practical note, it may be seen without a parametric study that a slot rotation angle of $45^{\circ}$ permits the longest possible slot for a $90^{\circ}$ slot inner angle and a maximum slot offset.

To ensure a large range of slot rotation angles could be studied, an offset of $5.0 \mathrm{~mm}$ was chosen while the slot length was maintained at $11.5 \mathrm{~mm}$. The calculated radiation and S-parameter performance are plotted below. 


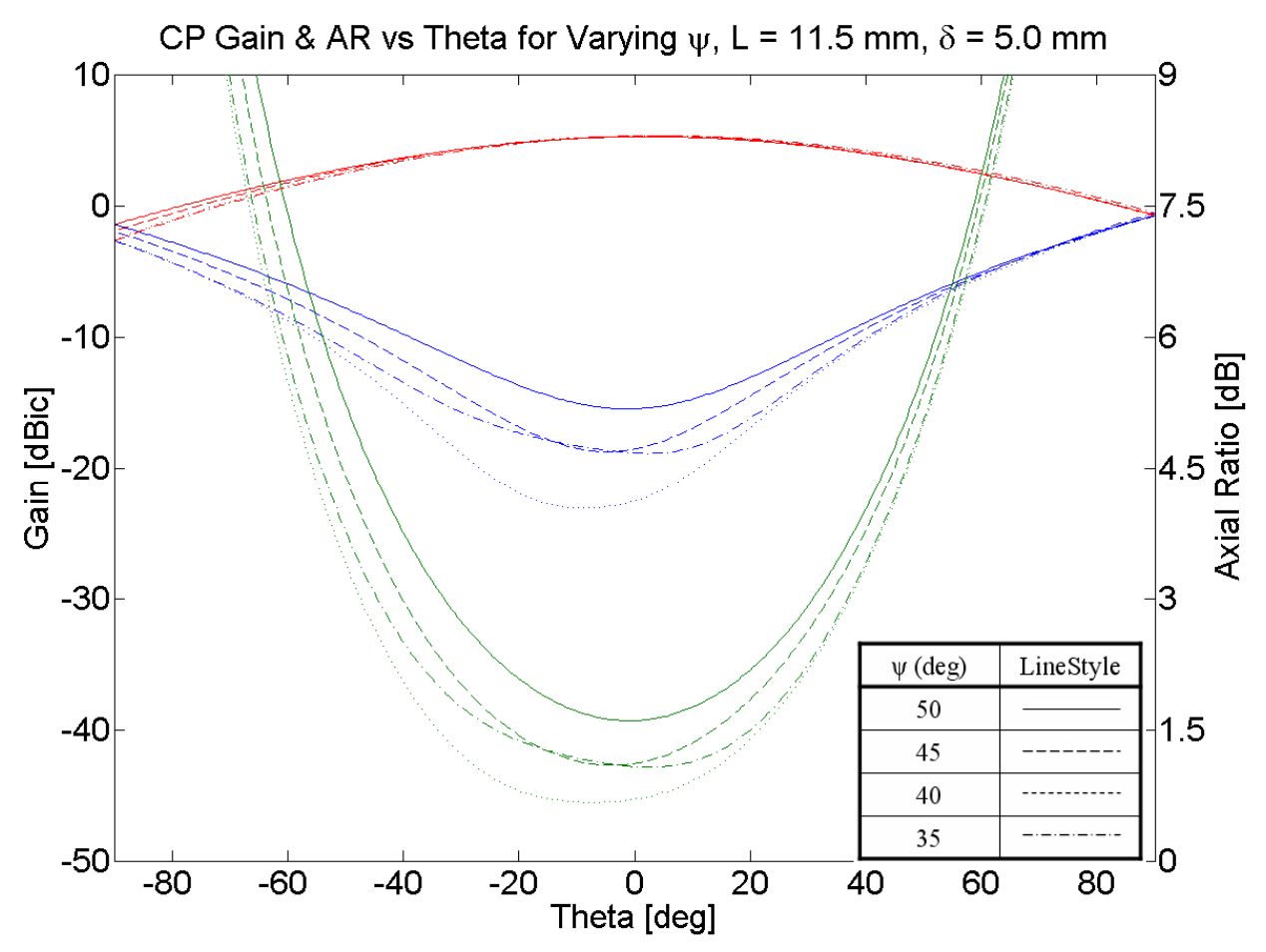

Figure 55 : 11.5 mm X-Slot, 12.5 GHz Radiation Performance vs. $\psi$

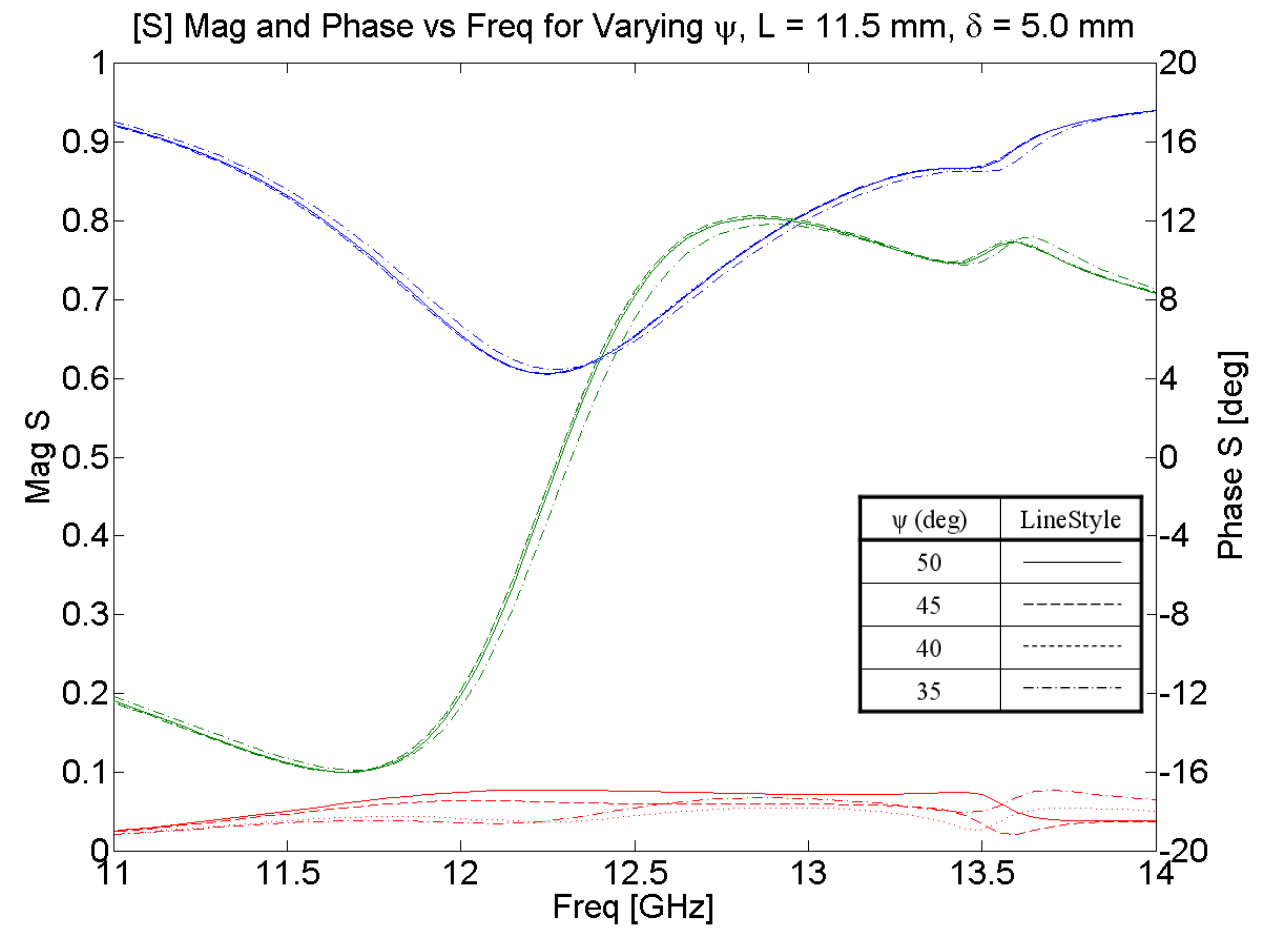

Figure 56 : 11.5 mm X-Slot, [S] vs. $\psi$ 
As anticipated, little relationship was observed between slot rotation angle and slot radiation or S-parameter performance. Curiously, the optimal return loss and axial ratio were observed for a slot rotation angle of $40^{\circ}$. This is considered of little consequence because the magnitude of the shift was small.

To conclude the parametric study of slot rotation angle, the anticipated lack of relationship between the design variable and slot performance was observed. The axial ratio, return loss, forward scattered phase, and radiated power maintained fairly constant values with up to $25 \%$ shifts in slot rotation angle. In terms of designing an $\mathrm{X}$-slot, it is recognized that a slot rotation angle of $45^{\circ}$ should generally be employed because it permits the maximum slot offset for a near-resonant slot length.

\subsection{X-Slot Parametric Study : $L_{1} / L_{2}$}

The effect of varying the ratio of slot lengths was studied next. It was anticipated that little good would come from an X-slot with unequal leg lengths, however, a paper by Ando and Hirokawa [1-17] presented an X-slot array where it was alluded to that each of the slot leg lengths controlled different aspects of X-slot performance. Ando suggested that the longer slot leg controlled the amount of coupled power, while the shorter leg length controlled the axial ratio. The context of the design discussion was a squinted beam traveling wave array application where the desired axial ratio minimum was not directly at zenith, but at some tilt angle (around $40^{\circ}-60^{\circ}$, typically).

The slot leg length ratio was studied by holding $\mathrm{L}_{1}$ constant at $11.5 \mathrm{~mm}$ and reducing $\mathrm{L}_{2}$ in regular steps to $9.5 \mathrm{~mm}$. The radiation pattern results and $\mathrm{S}$ parameter performance are found in Figure 57 and Figure 58, respectively. 


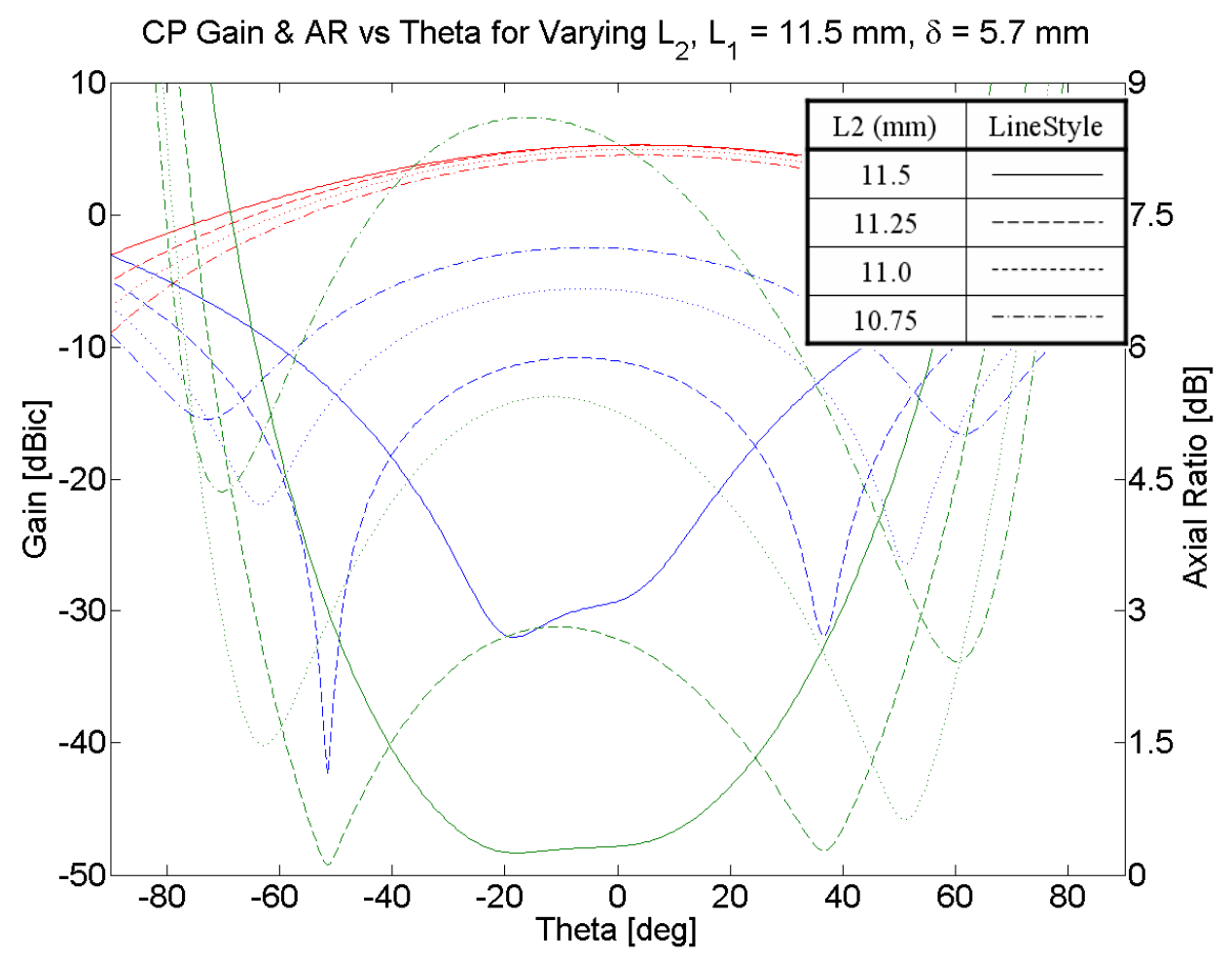

Figure 57 : 11.5 mm X-Slot, 12.5 GHz Radiation Performance vs. $L_{1} / L_{2}$

[S] Mag and Phase vs Freq for Varying $\mathrm{L}_{2}, \mathrm{~L}_{1}=11.5 \mathrm{~mm}, \delta=5.7 \mathrm{~mm}$

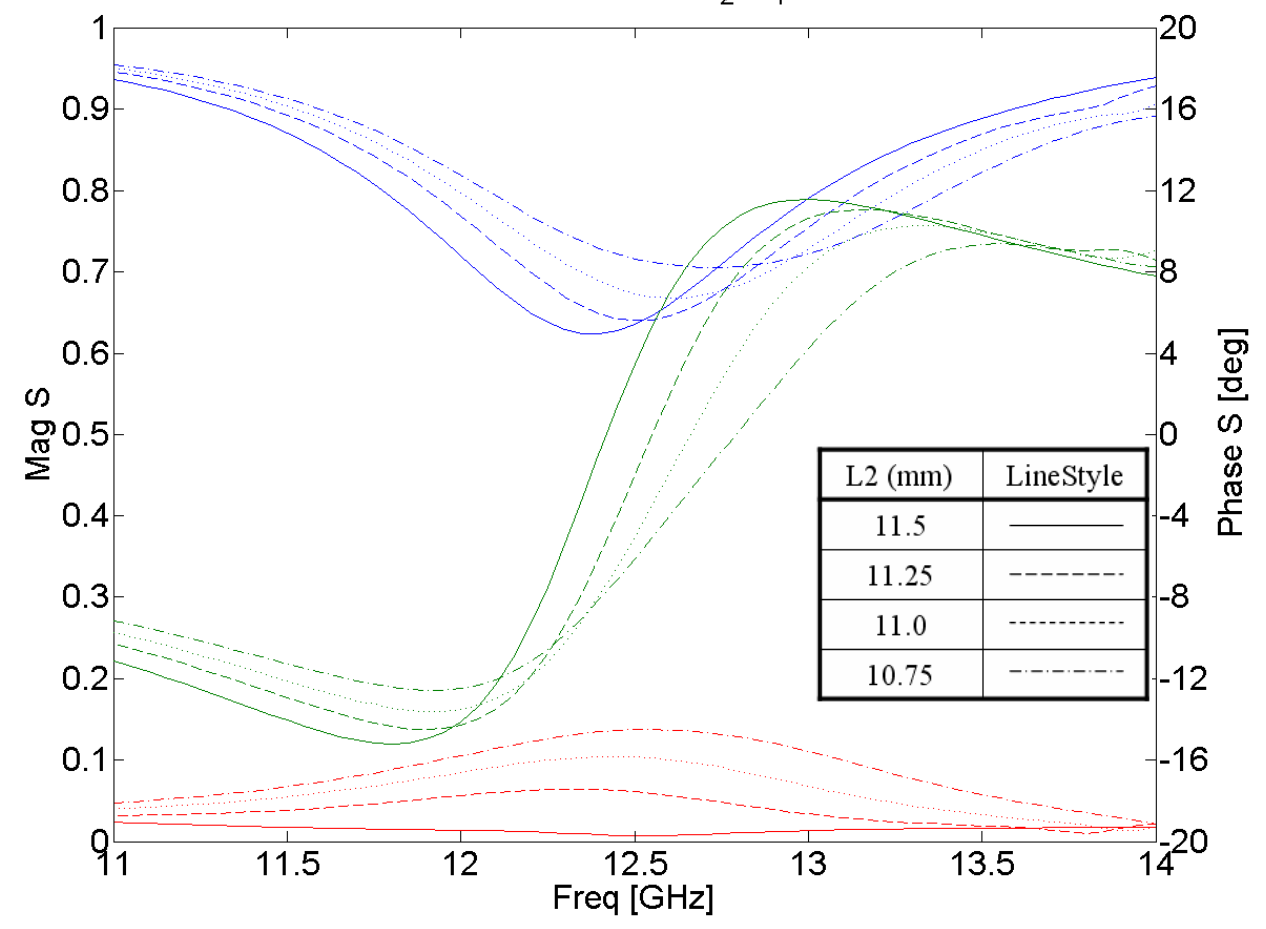

Figure $58: 11.5 \mathrm{~mm}$ X-Slot, $[S]$ vs. $\mathrm{L}_{1} / \mathrm{L}_{2}$ 
As anticipated, the axial ratio at zenith degraded with shifts in $\mathrm{L}_{2}$. A small $(0.5 \mathrm{~mm})$ shift in the slot length resulted in an axial ratio shift from around $0.5 \mathrm{~dB}$ to $5.0 \mathrm{~dB}$. The results also seemed to confirm Ando's paper. As the length of the shorter slot was reduced, the minimum axial ratio diverged from zenith and moved toward the horizon. For slight shifts in length, good axial ratio minima were observed out to $60^{\circ}$ in elevation, which is within the typical range of a tilted beam traveling wave array. The axial ratio performance vs. variations in the shorter slot length is plotted in Figure 59.

An interesting and insightful relationship was observed between return loss and the slot length ratio. As the slot length ratio was shifted away from unity, the return loss performance of the slot degraded. This may be observed, to a degree in Figure 58, above, but was plotted explicitly across the full range of tested $\mathrm{L}_{2}$ values in Figure 60. As with previous parametric studies, the relationship between axial ratio and return loss was observed.

Curiously, a very non-intuitive relationship was observed between $\mathrm{S}_{11}$ and $\mathrm{S}_{21}$. When the slot length ratio shifts from unity and the return loss increases, it would be expected that, because the slot is reflecting more energy, the amount of energy passed through the guide would decrease. This was not the case, however, as $\mathrm{S}_{21}$ actually increased when the return loss increased. Thus, when the slot length ratio shifted from unity it scatters more energy back toward the source and forward to the load, while radiating less energy. This result was not anticipated in Simmons' original model of the slot and was not predicted by any theories of slot operation available in the literature.

Taking a cue from the observed data and the consistent relationship between return loss and axial ratio performance in X-slot design variations, a new theory of operation was developed for the X-slot. This theory is, admittedly, beyond the direct scope of this work and is therefore relegated to Appendix A. 


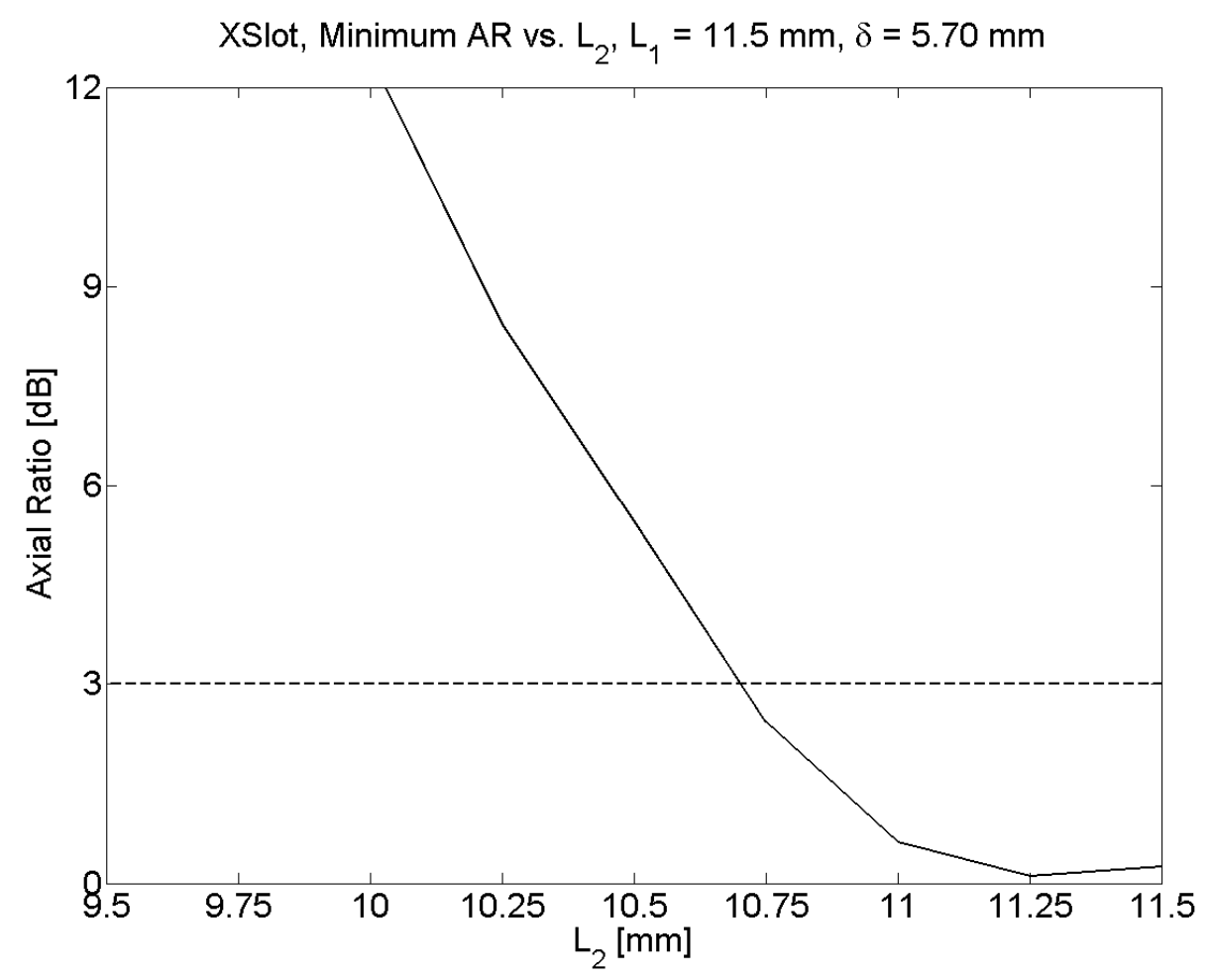

Figure 59 : 11.5 mm X-Slot, $12.5 \mathrm{GHz}$ AR vs. $\mathrm{L}_{1} / \mathrm{L}_{2}$

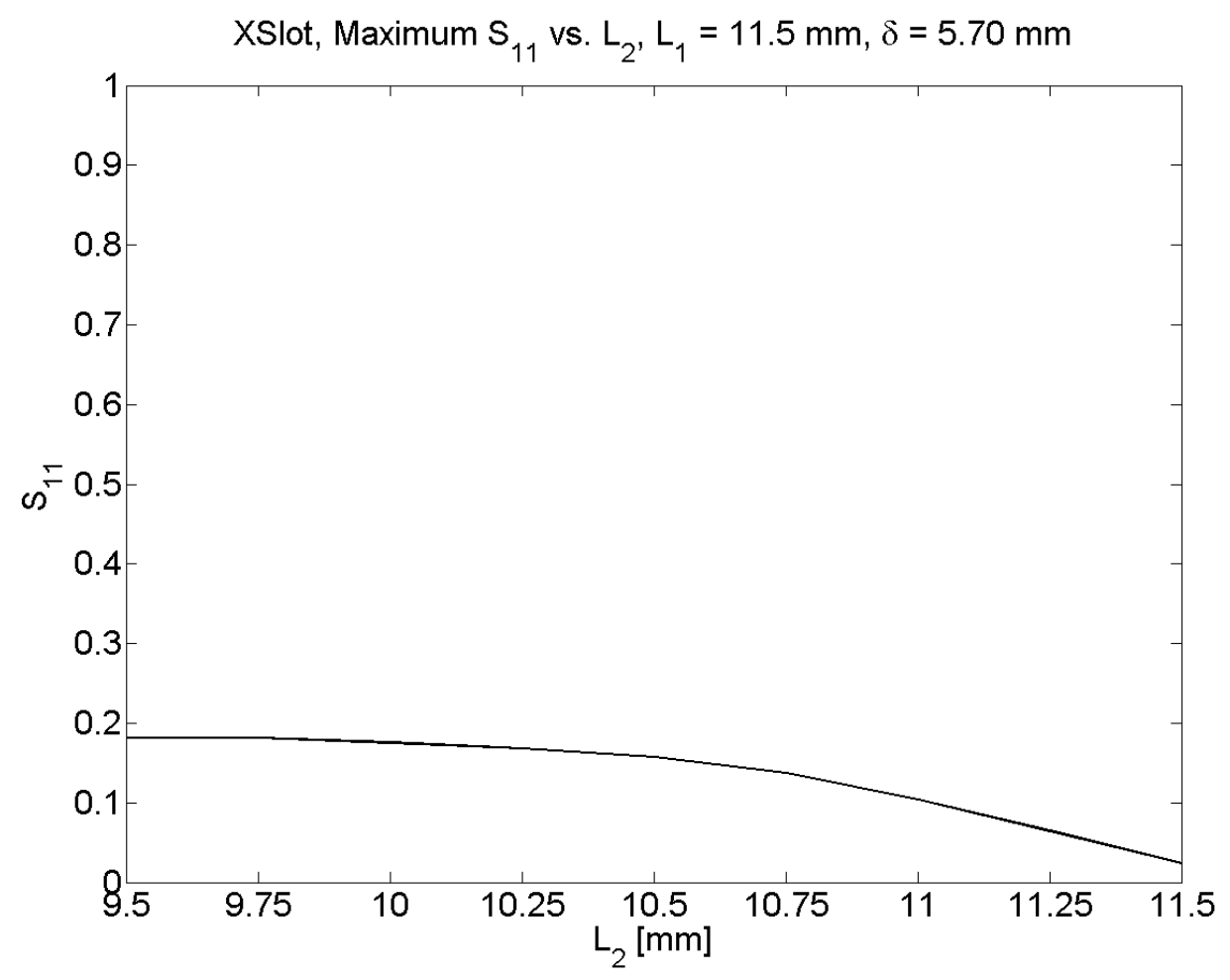

Figure 60 : $11.5 \mathrm{~mm}$ X-Slot, $\operatorname{Max} S_{11}$ vs. $L_{1} / L_{2}$ 
To conclude and summarize the slot length ratio parametric study, little value is gained by shifting this design parameter away from unity. If a tilted beam traveling wave array were to be designed, then the phenomena of a nonzenith minimum axial ratio could be exploited. However, within the context of developing a set of design guidelines for $\mathrm{X}$-slot radiators in a dual-CP linear array with broadside radiation, it is recommended that the slot length ratio be maintained at unity.

\subsection{X-Slot Parametric Study : w}

A parametric study on the X-slot width was conducted over a range from 0.25 $\mathrm{mm}$ up to $1.75 \mathrm{~mm}$. The limits were chosen from a slot thin enough to be difficult to machine, up to a slot width large enough to restrict the set of offsets and lengths available while remaining entirely within the waveguide broadside wall. While plots similar to those found throughout the remainder of this study were generated, none will be presented because no significant relationships were observed. That is, the radiation and S-parameter performance of the range of Xslots were virtually identical. The only recommendation that can be made relative to designing an $\mathrm{X}$-slot is to choose a slot width that is reasonably simple to machine. To be consistent, it is recommended that an accurate slot width is employed in the model, but the tolerances on the accuracy of the simulated value are significantly lower than other slot design parameters.

\subsection{X-Slot Parametric Study : $t$}

The final X-slot design variable to be studied parametrically was the waveguide wall thickness. To this point, all simulations were carried out with planar X-slots embedded in the wall of a waveguide defined purely in terms of conducting boundary conditions. Solid walls were introduced to the waveguide model, but 
otherwise all simulated conditions remained identical. The waveguide wall thickness was increased from $0 \mathrm{~mm}$ (the previous solution) to $2.5 \mathrm{~mm}$.

It was anticipated that as the waveguide wall thickness increased, the amount of energy stored within the slot field region would also increase, thereby changing the slot reactance. This was due to the evanescent, multi-mode field structure within the slot aperture. As the slot thickness increased, the amount of power radiated by these fields would decrease. Before starting the simulations, it was unclear whether the increased slot reactance would present itself as a simple shift in the frequency response of the slot or if the return loss would also increase.

Regarding the radiating fields, it was anticipated that the axial ratio and basic slot directivity patterns would remain mostly unchanged with shifts in waveguide wall thickness. All the slot performance relationships should be observed within the waveguide, not in the radiating space.

The slot radiation patterns and S-parameter performance for several values of wall thickness are plotted in Figure 61 and Figure 62, below. Little shift was observed in the radiation patterns, as expected. The S-parameter performance behaved within the scope of the anticipated response. As the waveguide wall thickness increased, the forward scattered phase and $S_{21}$ values changed. The net effect appeared to be that the slot appeared slightly shorter than it actually was, as the resonant frequency shifted upward. The return loss did not change, as would be expected from the broad band $\mathrm{S}_{11}$ data observed thus far. 


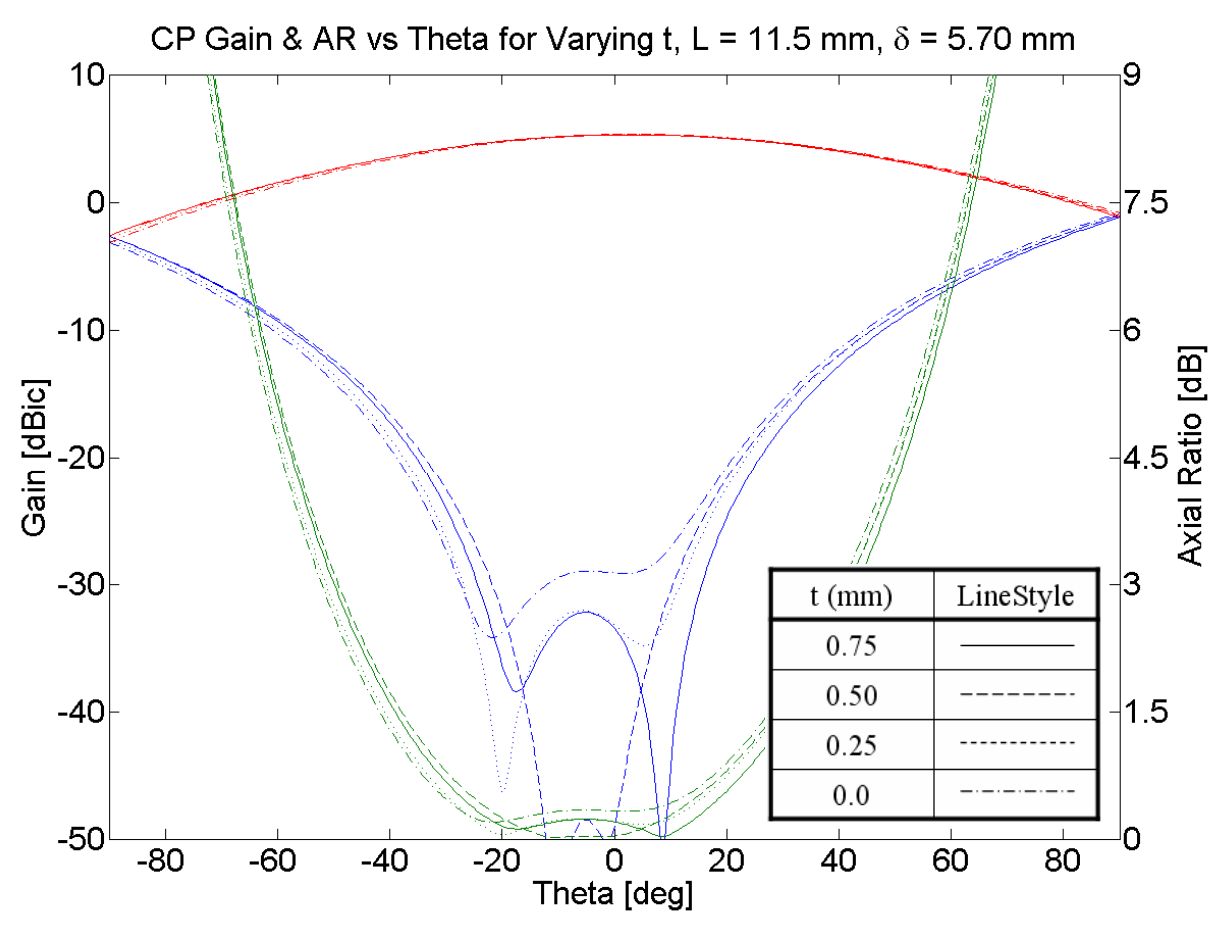

Figure 61 : 11.5 mm X-Slot, 12.5 GHz Radiation Performance vs. t

[S] Mag and Phase vs Freq for Varying $\mathrm{t}, \mathrm{L}=11.5 \mathrm{~mm}, \delta=5.70 \mathrm{~mm}$

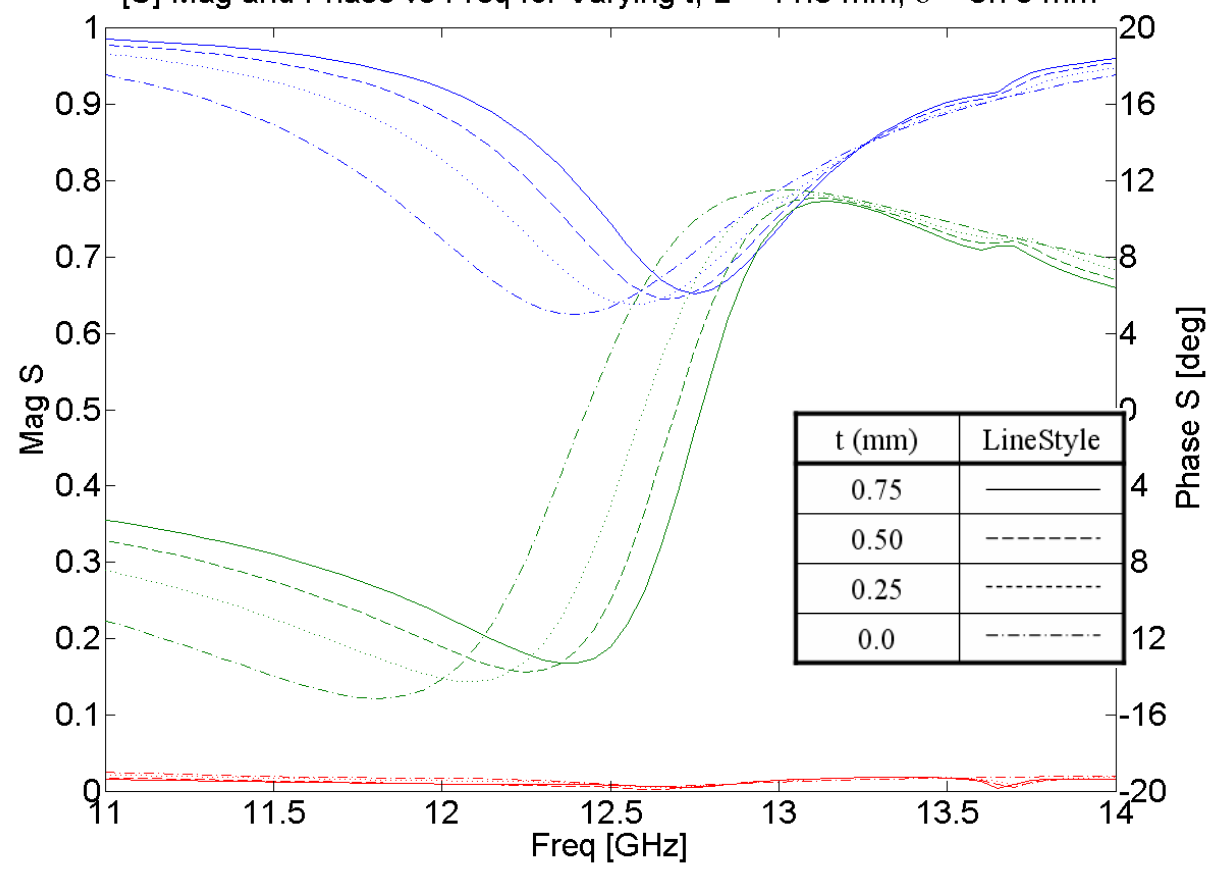

Figure $62: 11.5 \mathrm{~mm} \mathrm{X-Slot,} \mathrm{[S]} \mathrm{vs.} \mathrm{t}$ 


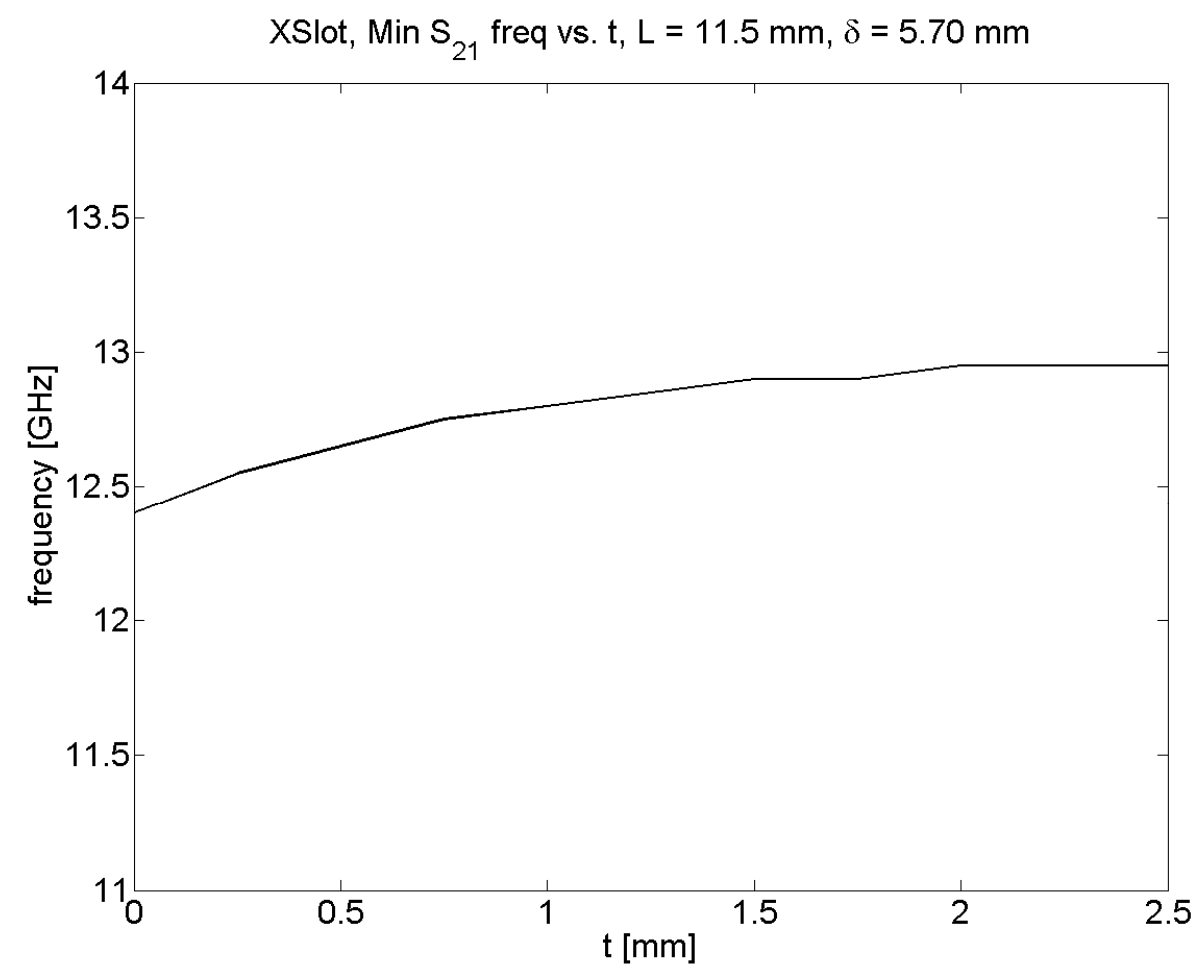

Figure 63 : $11.5 \mathrm{~mm} \mathrm{X-Slot,} \mathrm{f}_{\text {res }}$ vs. $t$

To further consider this relationship, the resonant frequency was plotted against the wall thickness over the full range of simulated values in Figure 63. The general trend approached a limit around $2 \mathrm{~mm}$.

The observed relationship between X-slot radiated power and forward scattered phase to waveguide wall thickness are not necessarily advantageous, but are noteworthy, nonetheless. In the proposed array design procedure, as discussed in Chapter 3, the $S_{21}$ magnitude value must be determined to within a prescribed range to achieve the desired aperture efficiency and percentage of load-terminated energy. If the wall thickness is not properly considered in the unit cell slot simulations, this value will be incorrectly estimated. Also outlined in Chapter 3 is the need to determine the forward scattered phase response of the X-slot antenna. This phase is not a design goal of the $\mathrm{X}$-slot, but rather is a piece of data from the $\mathrm{X}$-slot analysis which is absolutely necessary in the slow wave structure passive phase shifter design. Ideally the inter-element phase shift is a total of $360^{\circ}$. Each $\mathrm{X}$-slot will provide some of this phase shift and the remainder must be generated by the slow wave structure. 
To summarize the parametric study of the waveguide wall thickness, it must be considered in the design process, but does not appear to offer any performance advantages to the designer. Manufacturing considerations will typically determine a waveguide wall thickness. In order to account for the Sparameter perturbations that this will present, relative to a planar solution, the known wall thickness must be considered. If a variable wall thickness is anticipated due to manufacturing tolerances, the results of this study indicate a design in the asymptotic region, where the S-parameters change slowly with wall thickness variations, would be advantageous.

The observed influence of a manufacturing detail which is not advantageous to an $\mathrm{X}$-slot designer raises a generally valid point. If a generic piece of software is employed to model the unit cell performance of the X-slot and an attempt is made to accurately model the slot, then every manufacturingspecific artifact must be included. For example, if the slots will be milled out of a sheet of material, then the corner radii should be modeled. Enough variations exist on the theme of manufacturing artifacts to defy complete coverage within the this body of work, so the responsibility to account for them is left to the designer.

\section{$5.8 \quad X$-Slot Design Guidelines}

After completing a study of the design variables for the standard X-slot, a set of generic design guidelines may be generated. The assumption, stated previously and held throughout this work, is that an X-slot designer will optimize and characterize the slot radiation and S-parameter performance in a commercial computational EM software package which is capable of simulating generic structures with high accuracy. The tool employed throughout this work was HFSS, but none of the results are restricted to any of the exclusive abilities of HFSS. Other FEM, MoM, and FDTD codes should work equally well.

The following guidelines are tied to each of the parametric study results. 
1. The slot offset value calculated from Simmons' equation will work well, if a short enough slot length can be employed to accommodate the generally large offset. If the calculated slot offset will not permit the slot to fit entirely within the waveguide wall, then push the slot as close to the edge as seems reasonably possible to manufacture. This slot offset will provide optimal axial ratio performance and return loss.

2. The two legs forming the X-slot should be as close to orthogonal as possible. Using a slot inner angle other than $90^{\circ}$ is possible, but will not have any positive effects for a traveling wave array with broadside radiation.

3. The slot rotation angle should be $45^{\circ}$. Other values are possible, but this value will permit the maximum slot length and slot offset combination.

4. Slot length is the primary design variable. The slot length may be increased or decreased to tune the frequency response and achieve a particular $\mathrm{S}_{21}$ value at the design frequency. To optimize return loss and axial ratio, the length of each slot leg should be identical.

5. Waveguide wall thickness and other manufacturing details offer little advantage to the designer, but must be accounted for in the X-slot analysis. In particular, the forward scattered phase and magnitude of $\mathrm{S}_{21}$ will not generally be accurate if manufacturing artifacts (like a finite wall thickness) are ignored in the design procedure.

The design guidelines provided here should provide a sufficient list of rules to observe while optimizing an $\mathrm{X}$-slot for a particular $\mathrm{S}_{21}$ magnitude and noting the phase in a generic commercial computational EM software package. Despite the large number of potential design variables, the majority of them are benign and of little use to the slot designer. Within the context of the proposed array topology and design procedure, this is considered a positive result because it simplifies the radiating element design procedure. An example of this design 
procedure may be found in the final technical chapter, below, which presents and discusses a sample array design from start to finish. 


\section{Chapter 6: Array Design Methodology and Example}

Having proposed a linear array design approach in Chapter 3, developed a design methodology for the slow wave structure passive phase shifter in Chapter 4, and produced a set of generically applicable design guidelines for the radiating element in Chapter 5, a final task remains to complete the technical content of this work. This chapter addresses the finalized linear array design methodology and demonstrates its merits via a transparent, start-to-finish design example. As the financial budget for this work has been virtually non-existent, test articles were not able to be fabricated. Instead, the array performance simulated in HFSS was confirmed with CST, a commercial software package employing the FDTD method to solve generic computational electromagnetic problems. Good agreement was found between the two methods and both confirmed the array design methodology.

\subsection{Dual CP Linear Array Design Methodology}

As mentioned above, a linear array design methodology was proposed in Chapter 3. The purpose of proposing a design methodology before developing design approaches for the slow wave structure passive phase shifter and X-slot radiators was to frame the development. For example, because near-optimal array aperture distributions may be generated with a uniform set of slots and the aperture efficiency can be related to the percentage of input power terminated in a matched load, the X-slot design guidelines reflected this. Relationships between slot design variables and $S_{11}$ and $S_{21}$ were considered, while more traditional slot array design methodologies, such as developing equivalent circuit representations of the slot, were intentionally avoided as unnecessary embellishments. It was believed that whenever possible, a simple, functional solution should be developed, in lieu of an unnecessarily complicated one.

As stated repeatedly throughout this work, the design methodology is intentionally generic such that it may be accessible to any array designer wishing 
to take advantage of the unique characteristics of the proposed array topology. The design methodology is as follows:

1. Determine the array layout - i.e. the inter-element spacings. If the final topology is a linear array, this is simple; if a planar array composed of juxtaposed linear arrays, this step will include determining a waveguide width and wall thickness. If a circular aperture is to be approximated with juxtaposed linear arrays of varying length, the remaining design steps will be required for each unique linear array.

2. Calculate the required slot coupling ratio. From Figure 33 and (16), the required $\mathrm{S}_{21}$ can be determined from the number of slots in the linear array and the desired load termination power.

3. Design the X-Slot. Simulate the X-slot unit cell in a waveguide with inner dimensions and wall thicknesses identical to those in the real array. An initial length of slightly less than $\lambda_{0} / 2$ is a good starting point in the optimization process. The slot length is the primary design variable which may be adjusted to achieve the necessary $S_{21}$. The guidelines given in Chapter 5 should be followed to optimize axial ratio and return loss. Once the desired $\mathrm{S}_{21}$ value is achieved, the forward scattered phase response due to the slot must be calculated. This may be achieved by moving the calculated port phase planes to the center of the X-slot, thereby removing any phase effects due to propagation in the feed waveguide.

4. Design the Slow Wave Structure. Taking into account the X-slot forward scattered phase response and the array spacing, design the slow wave structure passive phase shifter to produce the necessary guided wave number to drive all the slot radiators in phase. The design methodology outlined in Chapter 4 should be followed to ensure an efficient, accurate slow wave structure solution where the 
desired guided wave number and return loss are simultaneously achieved.

5. Simulate the Linear Array. If computing resources permit, simulate the entire $\mathrm{N}$-element linear array to confirm the design process.

As will be seen in the next section, the first four steps in the design methodology are simple, given the right tools. Assuming a generic FEM, FDTD, or MoM solver is available for the X-slot design; as well as a software package optimized for waveguide structures (like the MM method) for the slow wave structure design, the entire design process should be well within the capabilities of a modern desktop PC (i.e. something on the order of a $3 \mathrm{GHz}$ processor with $1 \mathrm{~GB}$ of memory). Moreover, the entire solution should be achievable within one day of active design time.

\subsection{Dual CP Broadside Radiating X-Slot Array Design Example}

The intense processing requirements to simulate finite, electrically large antenna arrays prohibited a large design example. To demonstrate the design methodology effectiveness, it was decided that a five element array would be designed at X-band. The design goals found in Table 6 were chosen.

\begin{tabular}{|c|c|}
\hline$f_{0}, \lambda_{0}$ & $10 \mathrm{GHz}, 30 \mathrm{~mm}$ \\
\hline Axial Ratio & $3 \mathrm{~dB}$ \\
\hline Return Loss & $-20 \mathrm{~dB}$ \\
\hline Aperture Efficiency $(\eta)$ & $75 \%$ \\
\hline
\end{tabular}

Table 6 : Design Example Goals

The aperture efficiency was calculated by (34) with $\mathbf{G}$ being the co-polarized linear gain (including all feed losses) and $\mathbf{A}_{\mathbf{P}}$ the physical area occupied by the radiating elements. 


$$
\eta=\frac{G \lambda^{2}}{4 \pi A_{P}}
$$

\subsubsection{Step 1 : Array Layout}

The required array layout was chosen based on a series of simple array factor calculations to predict the aperture efficiency. The array layout values, as referenced to Figure 64, are captured in Table 7. Note that the array spacing in the direction of wave propagation is around $25 \%$ shorter than the guide wavelength. If the slow wave structure passive phase shifters were not present, a beam pointing about $56^{\circ}$ from zenith would be expected.

\begin{tabular}{|c|c|}
\hline$\Delta \mathrm{u}$ & $27 \mathrm{~mm}$ \\
\hline$\Delta \mathrm{v}$ & $27 \mathrm{~mm}$ \\
\hline Wall Thickness & $0.5 \mathrm{~mm}$ \\
\hline WG Width & $26.5 \mathrm{~mm}$ \\
\hline WG Height & $13.25 \mathrm{~mm}$ \\
\hline$\lambda_{\mathrm{g}}$ & $36.4 \mathrm{~mm}$ \\
\hline$\beta_{\mathrm{g}}$ & $172.66 \mathrm{rad} / \mathrm{m}$ \\
\hline
\end{tabular}

Table 7 : Design Example Array Layout 


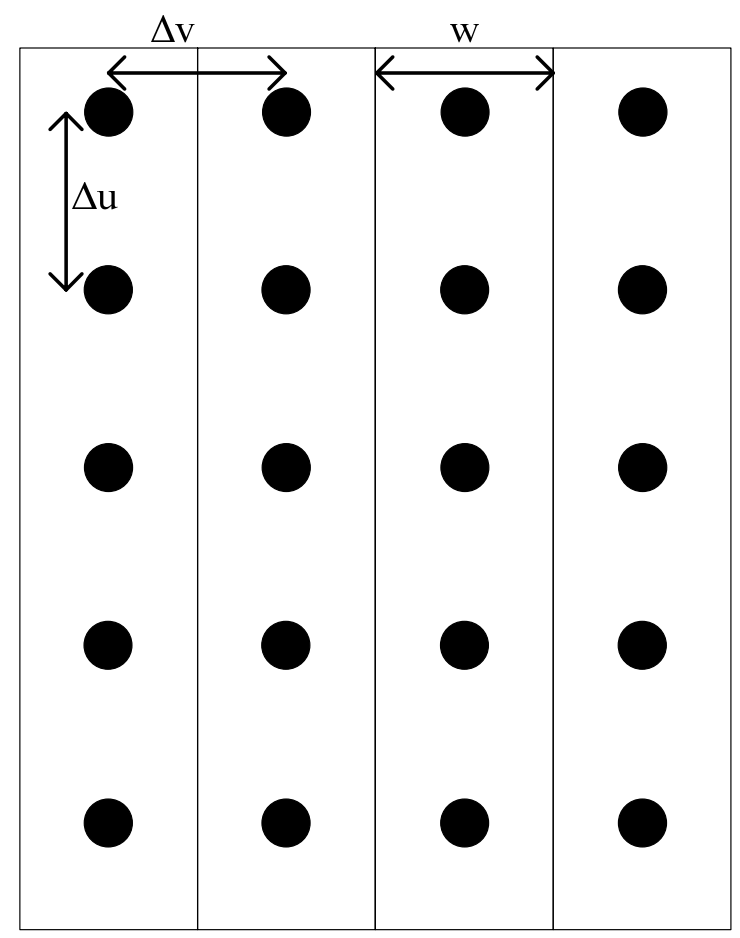

Figure 64 : Design Example Array Layout

\subsubsection{Step 2 : Slot Coupling Ratio}

To calculate the required slot coupling ratio, a range of load termination values was determined from Figure 33. The load termination value was entered into equation (16), to achieve a required $\mathrm{X}$-slot $\mathrm{S}_{21}$ value of 0.78

\subsubsection{Step 3 : X-Slot Design}

The waveguide inner width and guided wave number from Step 1, above, were entered into equation (10), to produce an ideal X-slot offset of $8.70 \mathrm{~mm}$. A slot length of $14 \mathrm{~mm}$ was chosen as an initial value slightly less than half of a free space wavelength. As anticipated, the ideal offset would not support this slot length entirely within the waveguide wall. An offset value of $7.90 \mathrm{~mm}$ was chosen in place of the ideal value to place the slot as close to the edge as possible. Based on the design guidelines given in Chapter 5, the slot rotation angle was set 
to $45^{\circ}$, the slot inner angle was $90^{\circ}$, and the lengths of both slot legs were set equal. Finally, a $0.5 \mathrm{~mm}$ slot width was chosen.

An initial simulation of the $\mathrm{X}$-slot, radiating into a half-space, required around 5 minutes of processing time in HFSS. S-parameter performance data is captured in Figure 65. The calculated $S_{21}$ at the design frequency, labeled in the figure, was found to be 0.84 . This was slightly higher than the desired value so a re-design was required. Based on the basic shape of the curve, it was decided to increase the length of the slot slightly, which should have had the effect of shifting all three plotted curves lower in frequency.

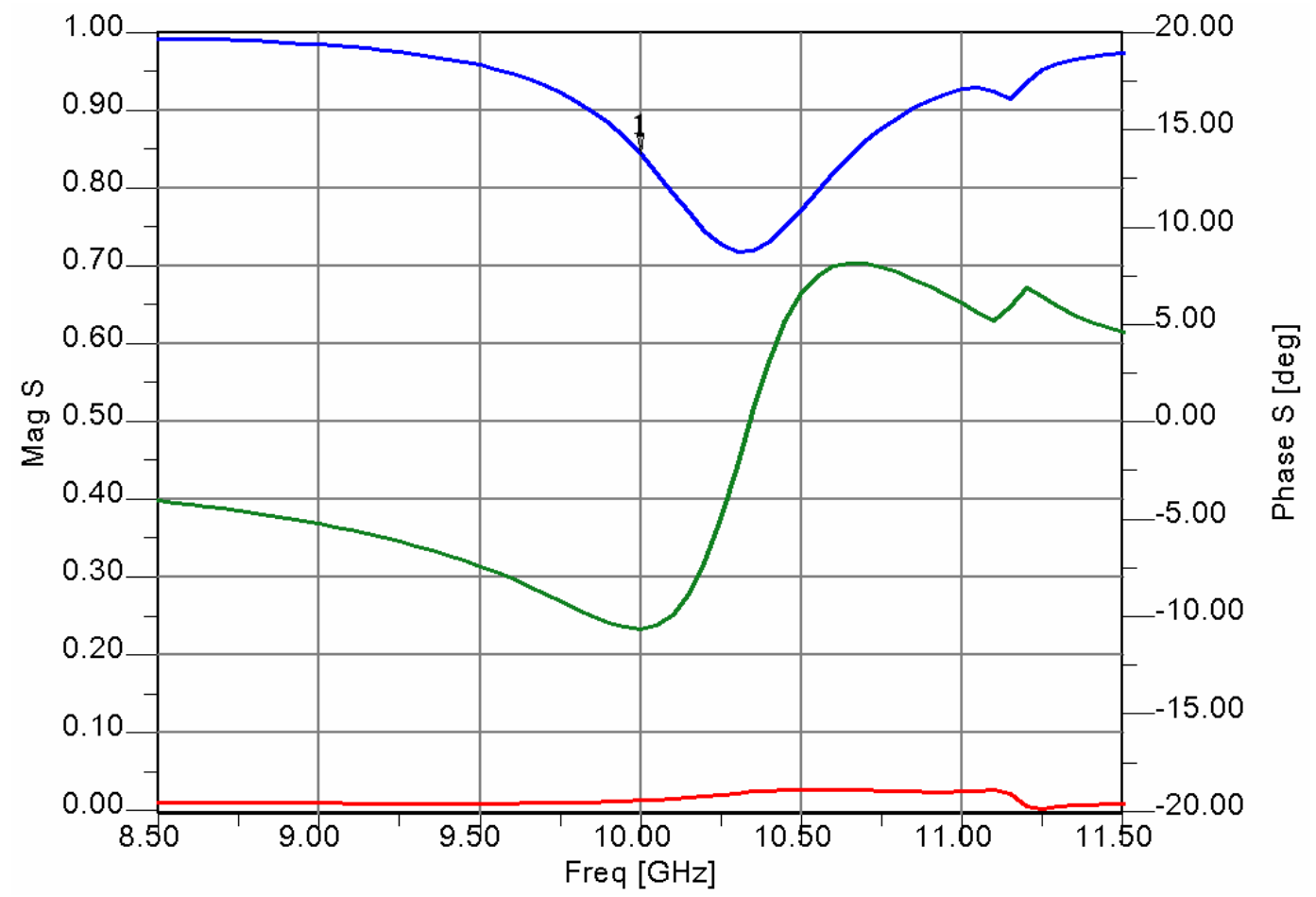

Figure 65 : Initial X-Slot, L = 14 mm, S-Parameters

After 3 additional iterations and around 15 minutes of simulation time, a satisfactory X-slot design was achieved with a slot length of $14.2 \mathrm{~mm}$. The Sparameter performance is captured in Figure 66. The calculated $\mathrm{S}_{21}$ for the optimized X-slot was 0.77 , which was deemed sufficiently close to the design goal of 0.78 because further refinement would require dimensional shifts smaller 
than the error tolerances of most standard machining practices. As expected, the $\mathrm{S}_{11}$ value was superb at 0.02 .

The radiation performance of the X-slot was confirmed and is captured in Figure 67. The axial ratio of $1.12 \mathrm{~dB}$ was below the design goal and the general radiation pattern exhibited the anticipated shape, thus it was considered acceptable.

Finally, before continuing on to Step 4 and designing the slow wave structure passive phase shifter, the forward scattered phase of the X-slot was calculated to be $-9.77^{\circ}$. This value was taken as a data point to be utilized in the next design step.

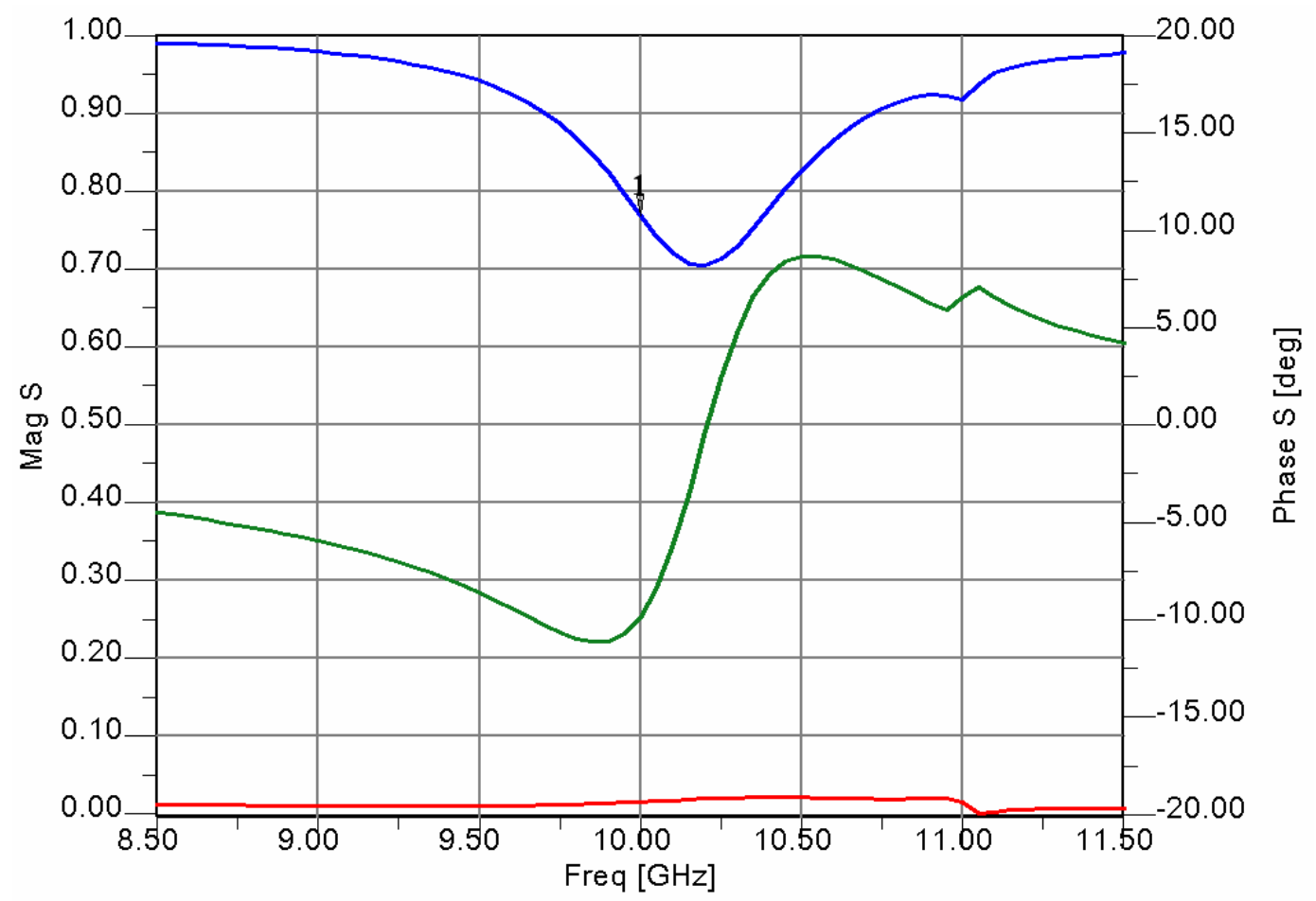

Figure 66 : Optimized X-Slot, $L=14.2 \mathrm{~mm}$, S-Parameters 


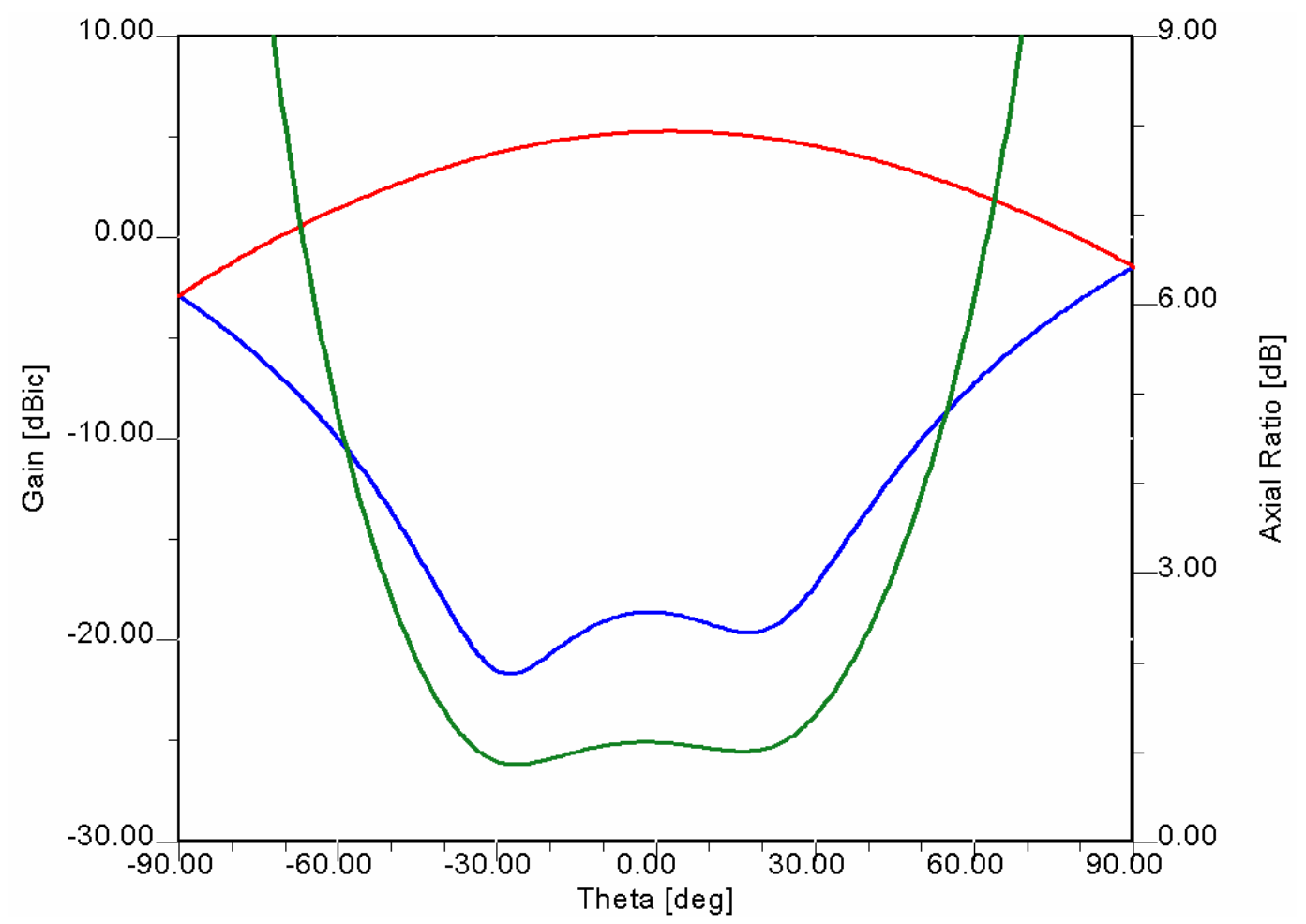

Figure 67 : Optimized X-Slot, $L=14.2$ mm, Radiation Patterns

\subsubsection{Step 4 : Slow Wave Structure Design}

The design steps outlined in Chapter 4 were next carried out to arrive at a slow wave structure passive phase shifter capable of good return loss performance and the necessary phase shift to produce a broadside radiation pattern from the linear array of X-slots. In order to account for the forward scattered phase shift of the $\mathrm{X}$-slot elements, the previous equation (19) describing the necessary guided wave number of the slow wave structure, had to be modified slightly:

$$
\beta_{c}=\frac{2 \pi-\Delta \phi_{21}}{\Delta u}
$$

In the new equation (34), $\Delta \boldsymbol{\Phi}_{21}$ is the forward scattered phase response of

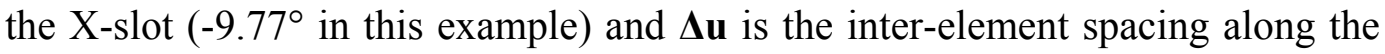
linear array, in the direction of wave propagation within the feed waveguide. Using this equation, a design goal of $226.4 \mathrm{rad} / \mathrm{m}$ was calculated. Following the design guidelines in Chapter 4 , an initial guess of $\mathbf{N}=3$ was chosen for the slow 
wave structure, meaning the initial inter-element spacing was set at $9 \mathrm{~mm}$. The Collin (27) and Marcuvitz (28-30) expressions were then employed to calculate an initial iris height of $5.77 \mathrm{~mm}$. Although the impact of finite thickness irises on the slow wave structure design procedure was never fully studied, a width of 0.5 $\mathrm{mm}$ was chosen as a reasonable approximation to an infinitely thin sheet because the Marcuvitz expressions employ this approximation.

A simulation was performed in HFSS with the initial set of iris dimensions. This step required around 5 minutes of processing time after the model was generated. The calculated return loss and wave number characteristics are captured in Figure 68 and Figure 69, respectively.

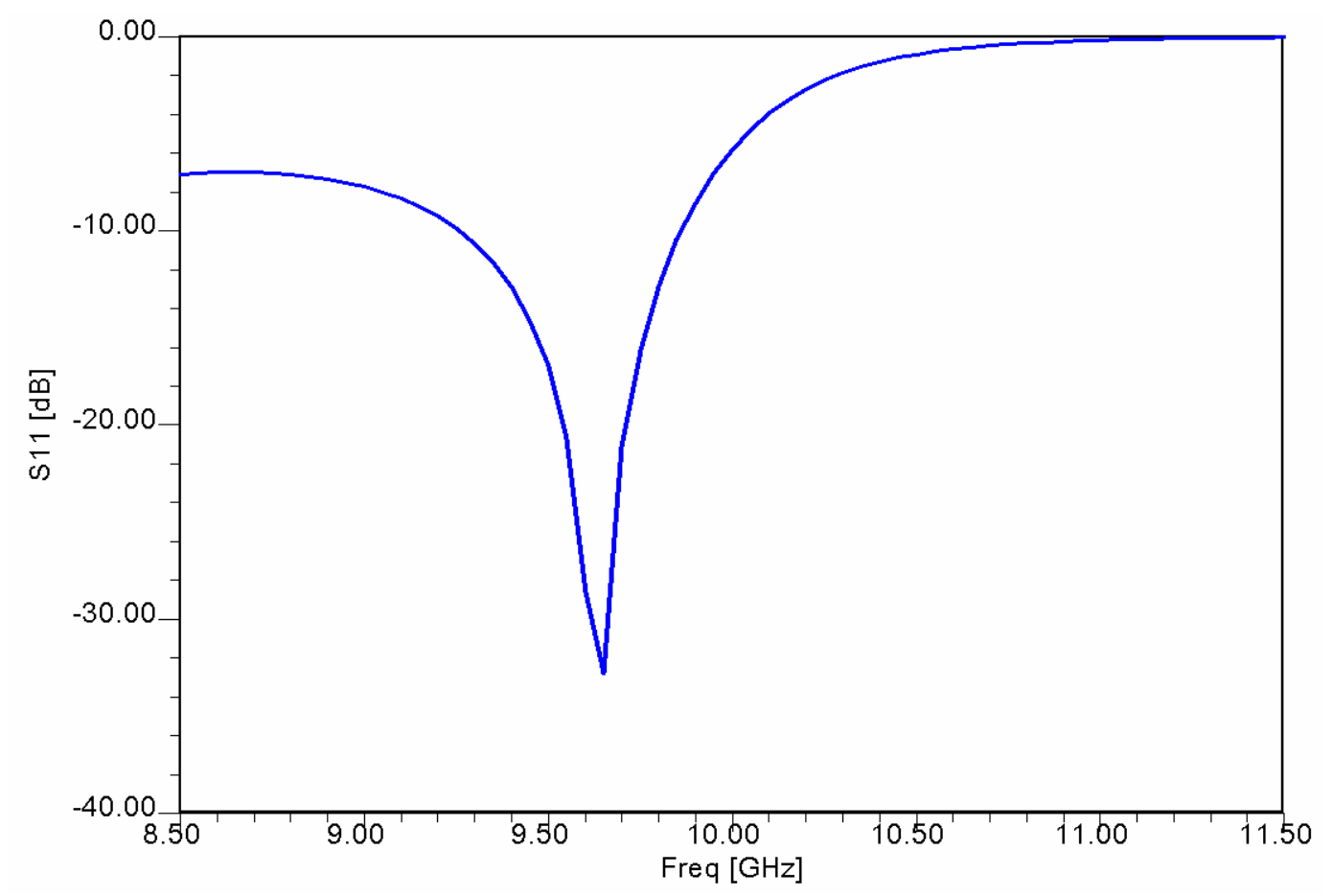

Figure 68 : Initial SWS Return Loss 


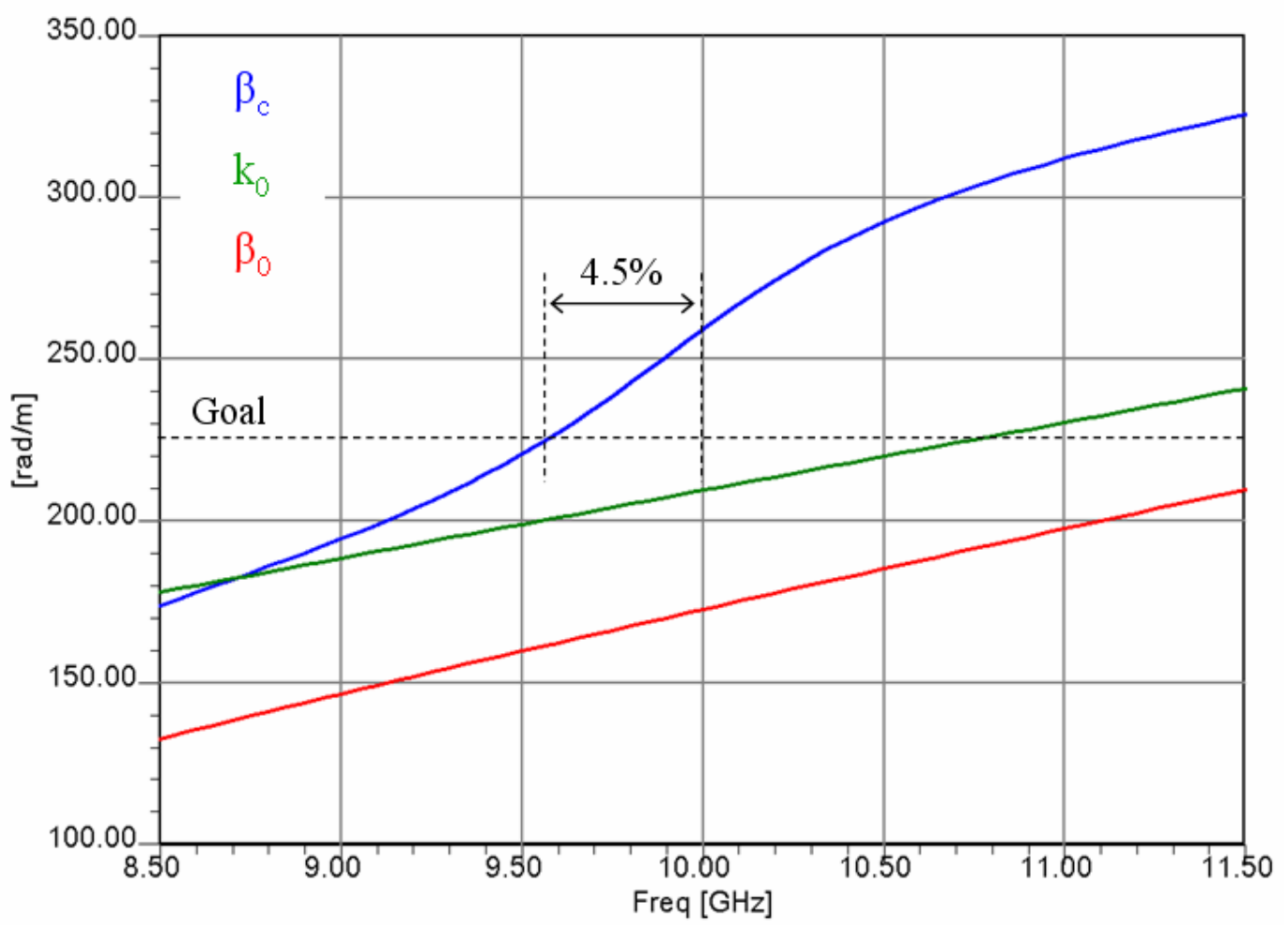

Figure 69 : Initial SWS Wave Number

As expected from previous experience, the calculated results exhibited non-optimal performance. The return loss was poor at $10 \mathrm{GHz}$ and the guided wave number design goal was achieved at $9.57 \mathrm{GHz}$. The $4.5 \%$ frequency shift in wave number would cause an unacceptable $13^{\circ}$ beam tilt.

As suggested in the design procedure, the problem was next handed over to a piece of software which has been optimized for waveguide structures via the Mode-Matching technique. As has been stressed throughout this work, several commercial software packages exist which can tackle these jobs, but an efficient designer will choose one that is optimized for the task at hand. In this example, that software was WASP-NET. A screen capture from the file setup in this program is depicted in Figure 70. 


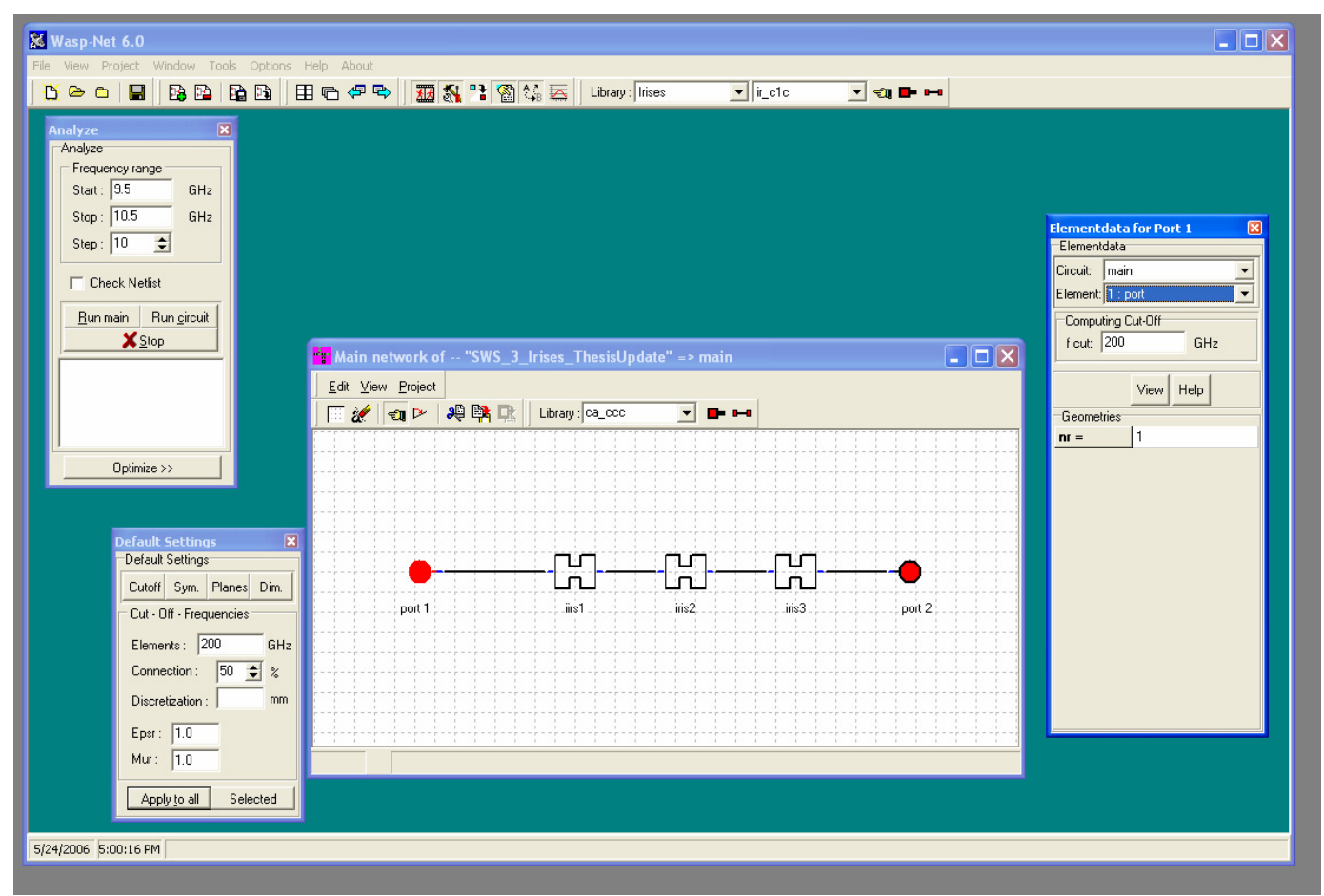

Figure 70 : WASP-NET Program Window

Within WASP-NET, an optimization algorithm employing Powell's method was given the design goals of at least $-30 \mathrm{~dB}$ return loss and a guided wave number of $226.4 \mathrm{rad} / \mathrm{m}$. These goals had to be met simultaneously at 10 $\mathrm{GHz}$. In the design space, the optimizer was allowed to alter the height of and separation between the irises. Two heights were permitted, with the outer irises being identical but possibly different from the central iris. The initial conditions for the design variables were taken from the design simulated in HFSS, above, based on the Collin/Marcuvitz solution.

After 43 optimization steps, requiring around 2 minutes of simulation time, an optimal solution was achieved in WASP-NET, with an iris spacing of 4.8 $\mathrm{mm}$, a central iris height of $6.9 \mathrm{~mm}$, and an outer iris height of $5.2 \mathrm{~mm}$. To confirm the results of the optimizer, the structure was simulated in HFSS (requiring an additional 5 minutes). The return loss and wave number performance are plotted below. 


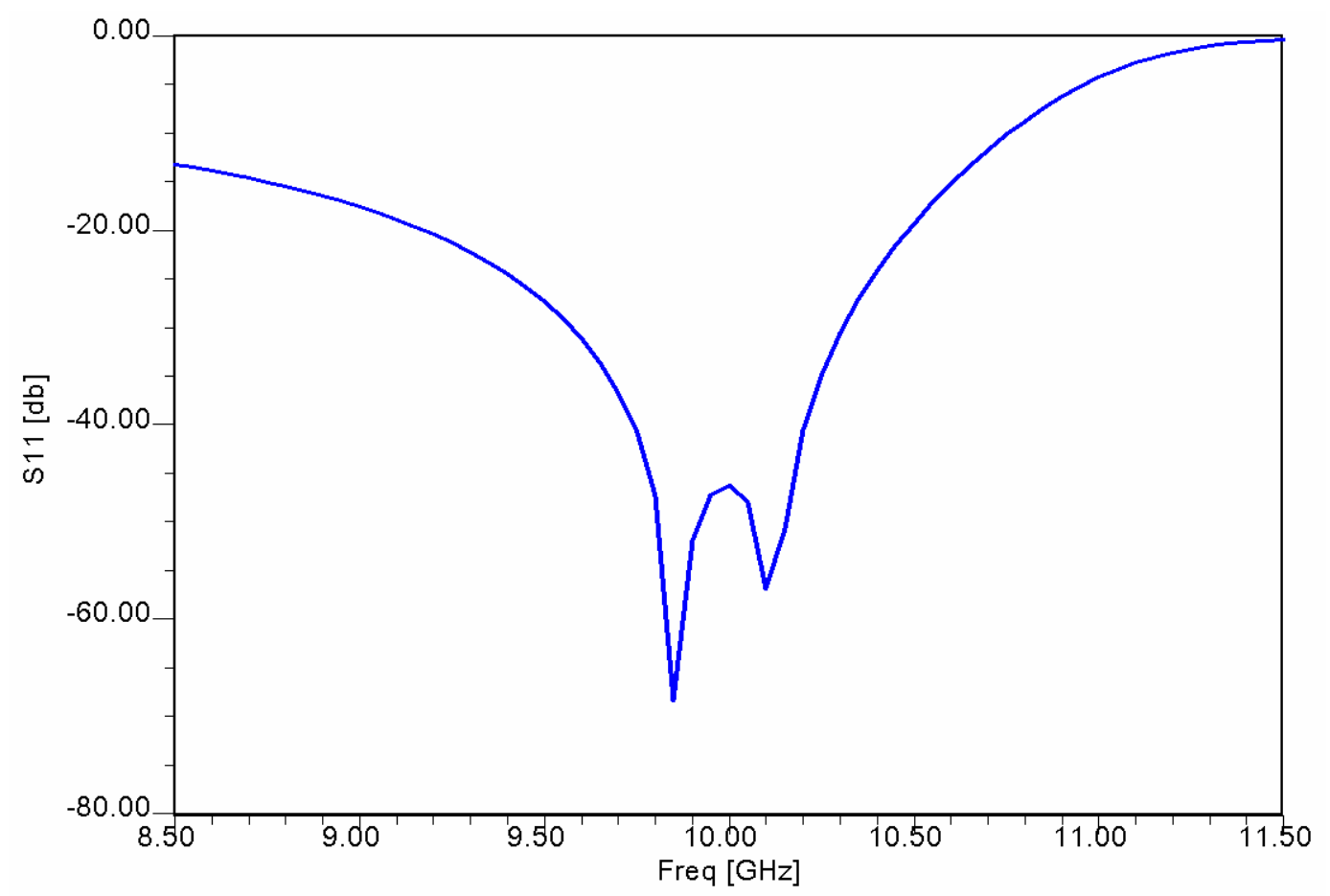

Figure 71 : Optimized SWS Return Loss

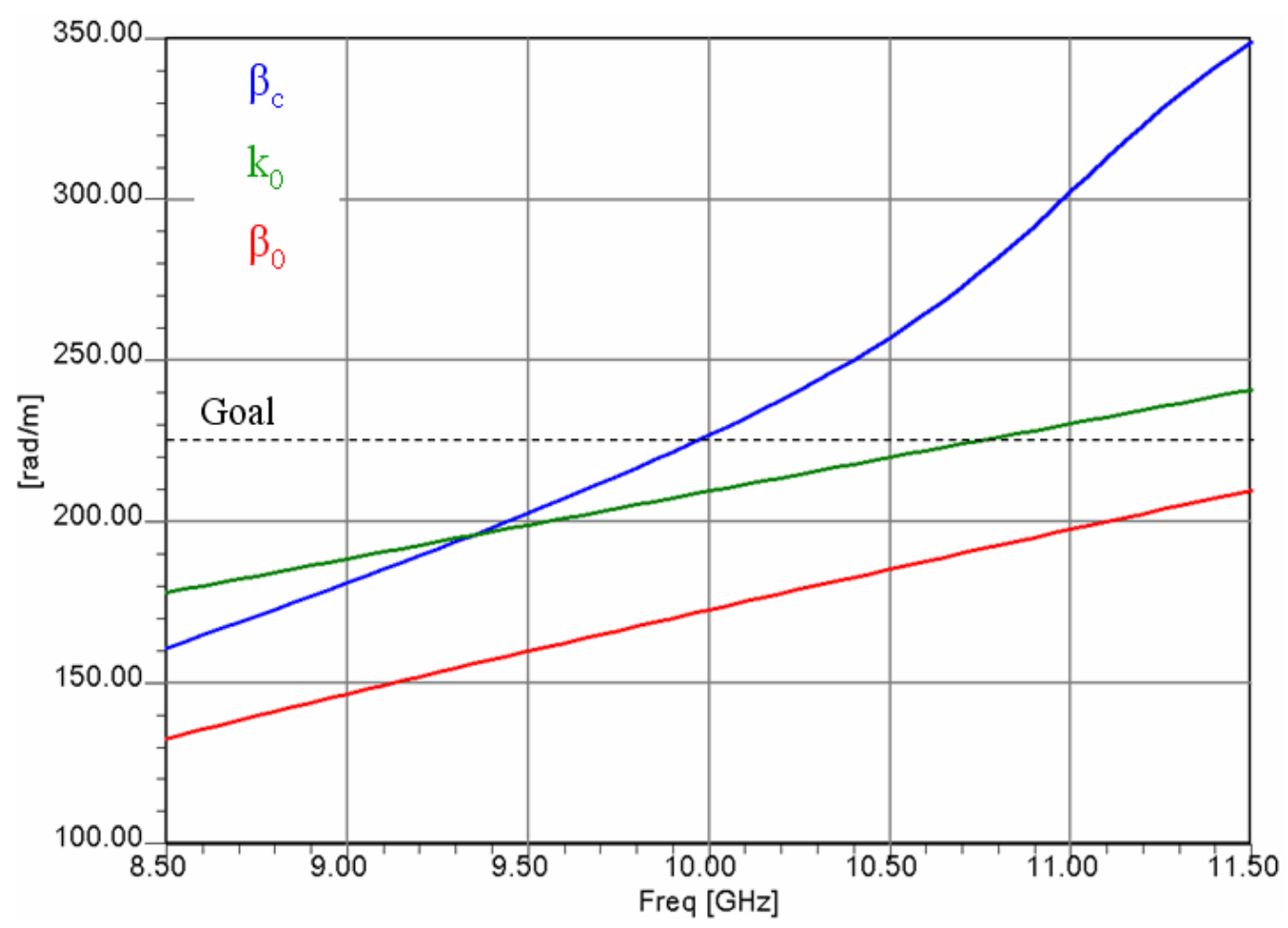

Figure 72 : Optimized SWS Wave Number 
The $S_{11}$ value of $-46.1 \mathrm{~dB}$ and guided wave number of $226.9 \mathrm{rad} / \mathrm{m}$ were considered exceptionally good results and no further optimization was required. The slow wave structure passive phase shifter design was complete and, thanks largely to the efficiency of WASP-NET, required less than 20 minutes of processing time. The model construction time was not explicitly captured in this report, but a designer fluent in the chosen analysis tools should be able to complete this process in less than 1 hour.

\subsubsection{Step 5 : Simulate the Linear Array}

The final step in the dual CP linear array design process was confirmation. Having determined the array layout, the X-slot design, and the slow wave structure passive phase shifter design, the array design work was complete, making this final step optional. In the design example, a five element array was chosen, making the task of simulating the full linear array not unreasonable. If, by contrast, an array of twenty elements were to be designed, this final step would probably not be an option with modern computing resources.

As mentioned above, simulations were carried out in both HFSS and CST. The FEM and FDTD methods were used to achieve result confirmation via agreement between philosophically orthogonal simulation techniques. Due to the superior flexibility of the tool, the majority of the array analysis was performed in HFSS, while CST was relied upon for simple result confirmation.

\subsubsection{The HFSS Model}

A set of images from the model constructed in HFSS, are found in Figure 73. Note that the X-slots are all identical and offset as far as possible within the waveguide wall. Also, the slow wave structure passive phase shifters are uniform, have two different height irises per unit cell, and are not distributed uniformly throughout the distance between X-slots. The finite thickness of the top waveguide wall was modeled, while the other three walls were established via 
PEC boundary conditions. The irises were solid metal objects with infinite conductivity and the X-slots were lossless vacuum. Not shown in the images is the infinite ground plane, level with the waveguide top wall, as well as the vacuum box terminated in a PML, into which the slots radiated. The array radiation patterns were calculated over the face of the air box surrounded by PML. The source fields were generated at either end of the waveguide on the faces transverse to the direction of propagation.
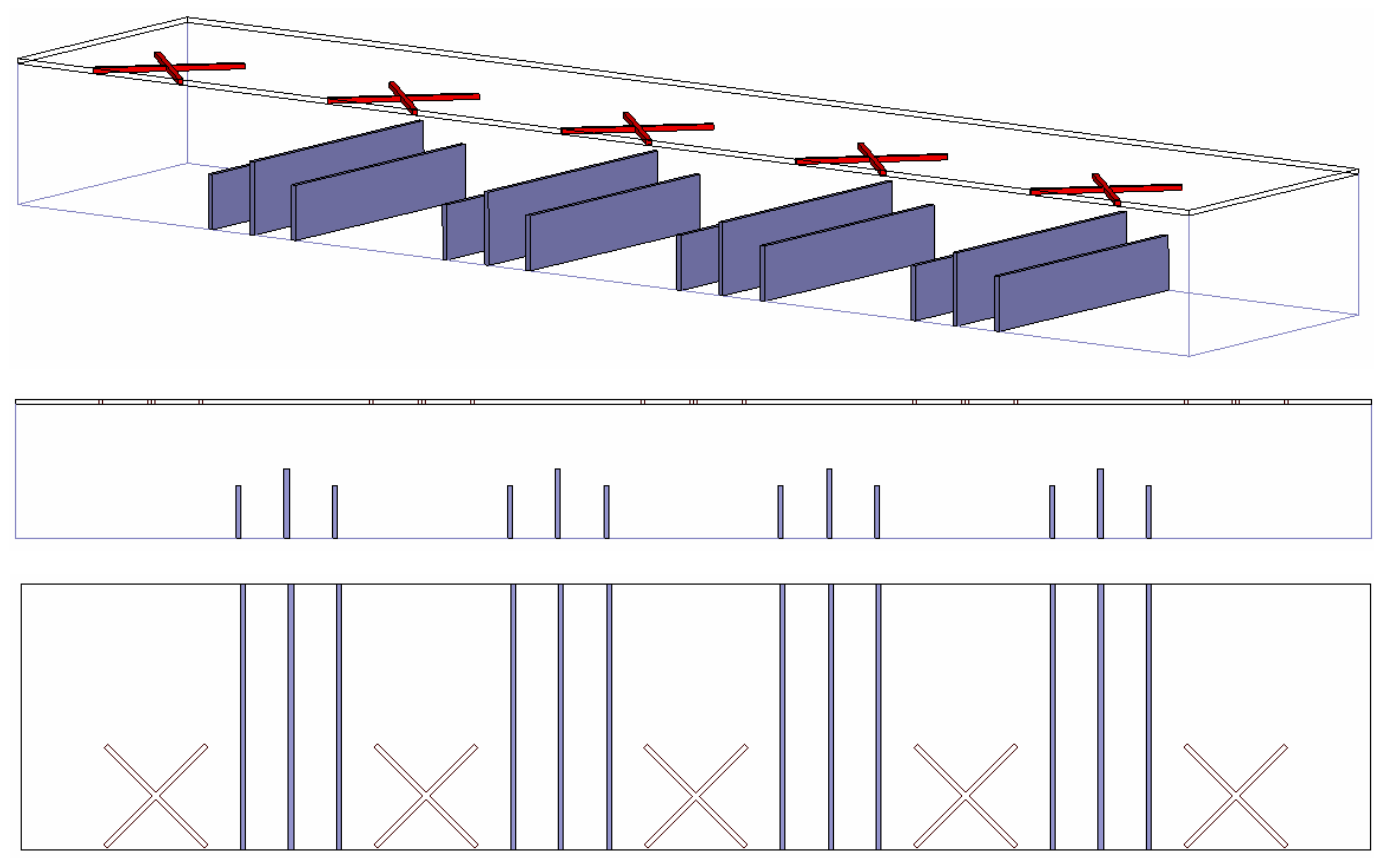

Figure 73 : HFSS Linear Array Design Example Model

A standard HFSS adaptive solution was forced to converge until the Sparameters between each pass varied by no more than 0.01 at the design frequency. Data across the 9.5 to $10.5 \mathrm{GHz}$ frequency band was generated via a discrete sweep with solutions every $100 \mathrm{MHz}$. The adaptive solutions required around 35 minutes of processing time and $3.2 \mathrm{~GB}$ of memory on an XP-64 workstation with four $2.2 \mathrm{GHz}$ Intel processors. The discrete sweep required a little over 2 hours and an identical amount of memory to complete. 


\subsubsection{The HFSS Array Performance at $10 \mathrm{GHz}$}

The calculated radiation patterns determined from an excitation at each port are seen in Figure 74 and Figure 75. Only one port was permitted to operate as a source at a time with the inactive port effectively terminated in a matched load. As anticipated in the design procedure, the array produced a broadside radiation pattern without grating lobes. Moreover, the RHCP and LHCP beams generated from either source are co-located at zenith.

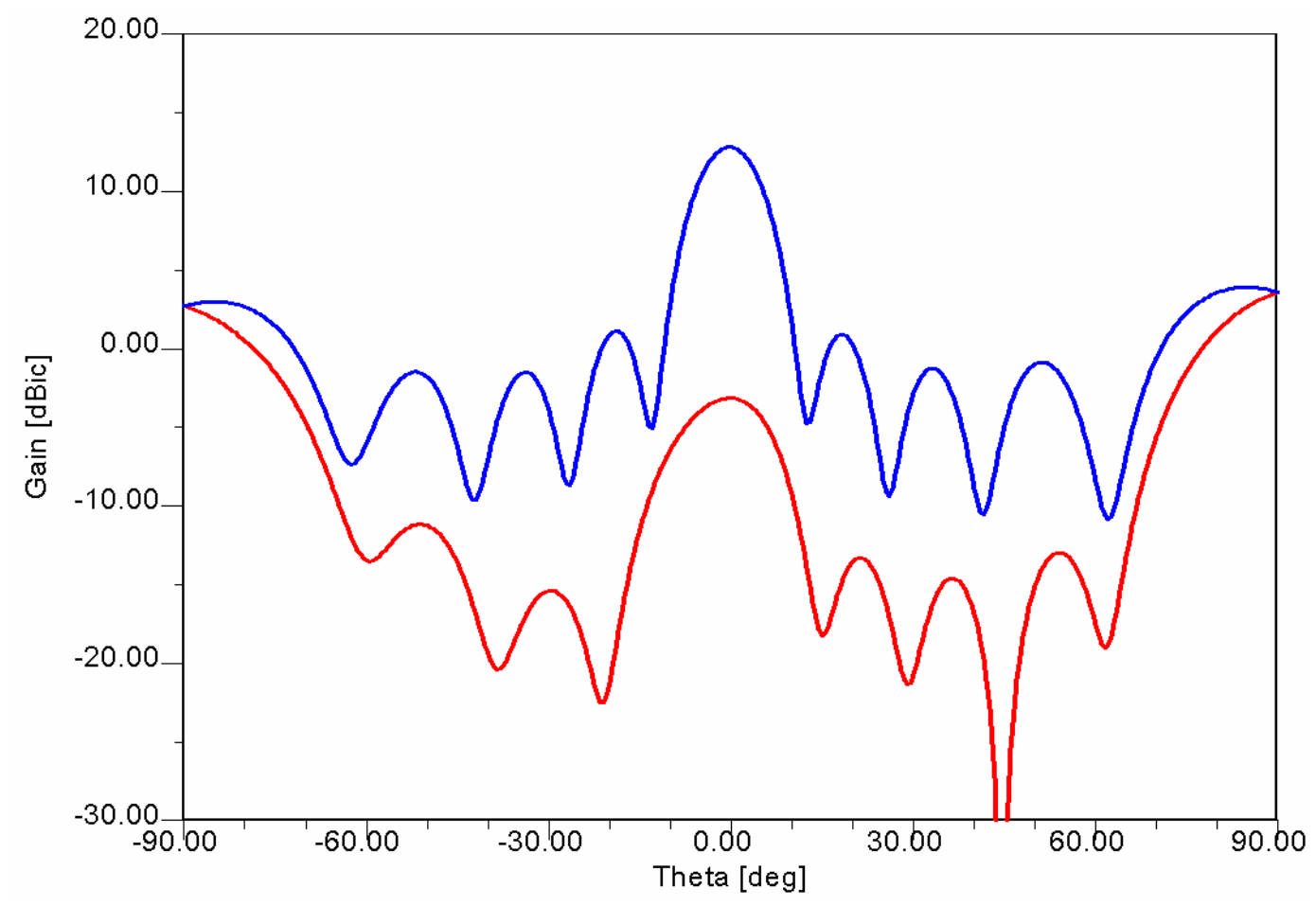

Figure 74 : Linear Array, Port 1 Radiation Patterns 


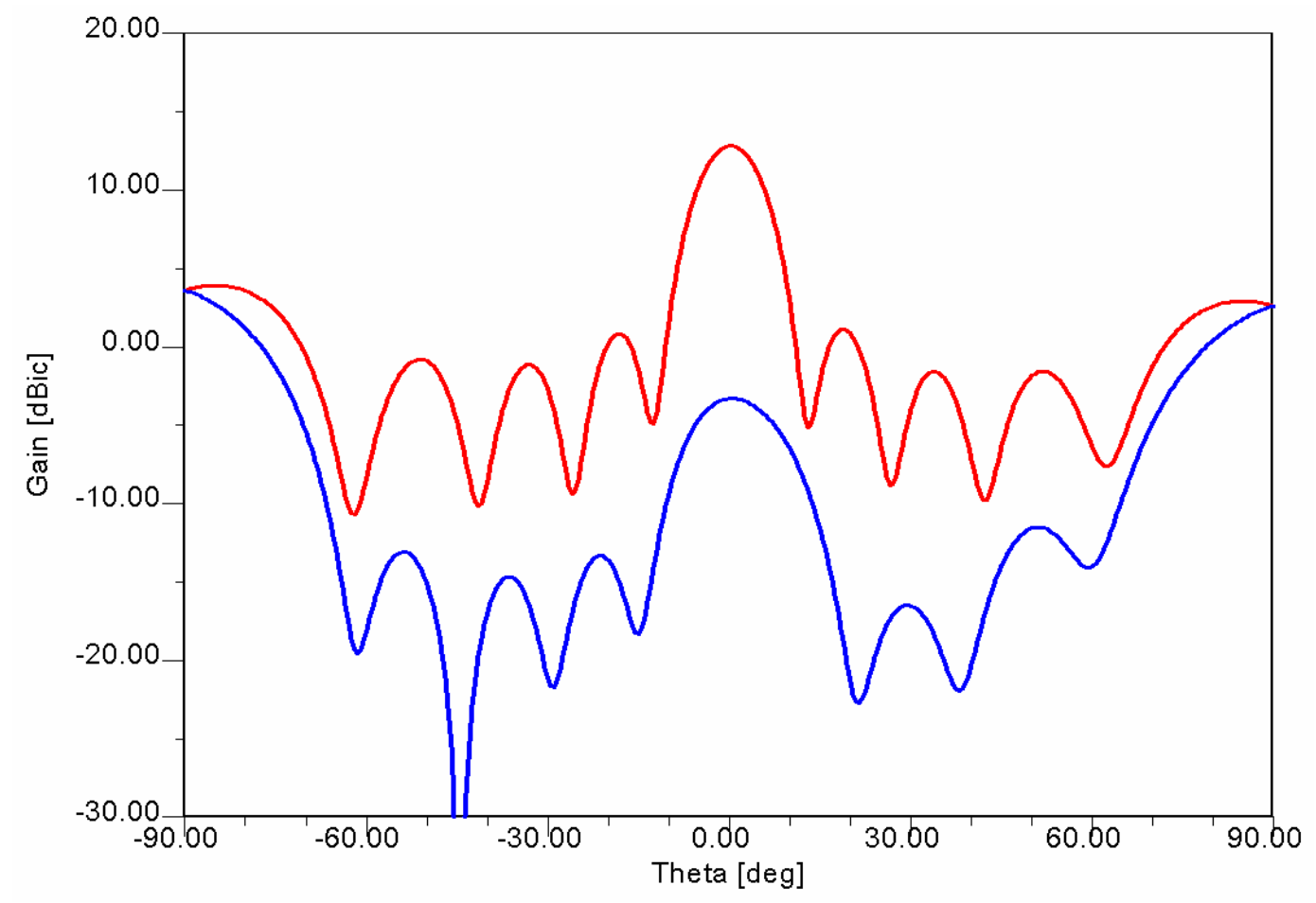

Figure 75 : Linear Array, Port 2 Radiation Patterns

As with previous radiation patterns in this work, the LHCP gain data is shown in blue, while the RHCP gain data is shown in red. The co-polarized and cross polarized gain were calculated across the hemisphere and mapped to a circle, as was done in Chapter 3 for the radiating element comparison. The full hemispherical patterns (Figure 76 and Figure 77) exhibit similar trends as the linear plots above. A single main beam is seen, at broadside; zero grating lobes are exhibited; and good cross polarization rejection is observed throughout the calculated region. 

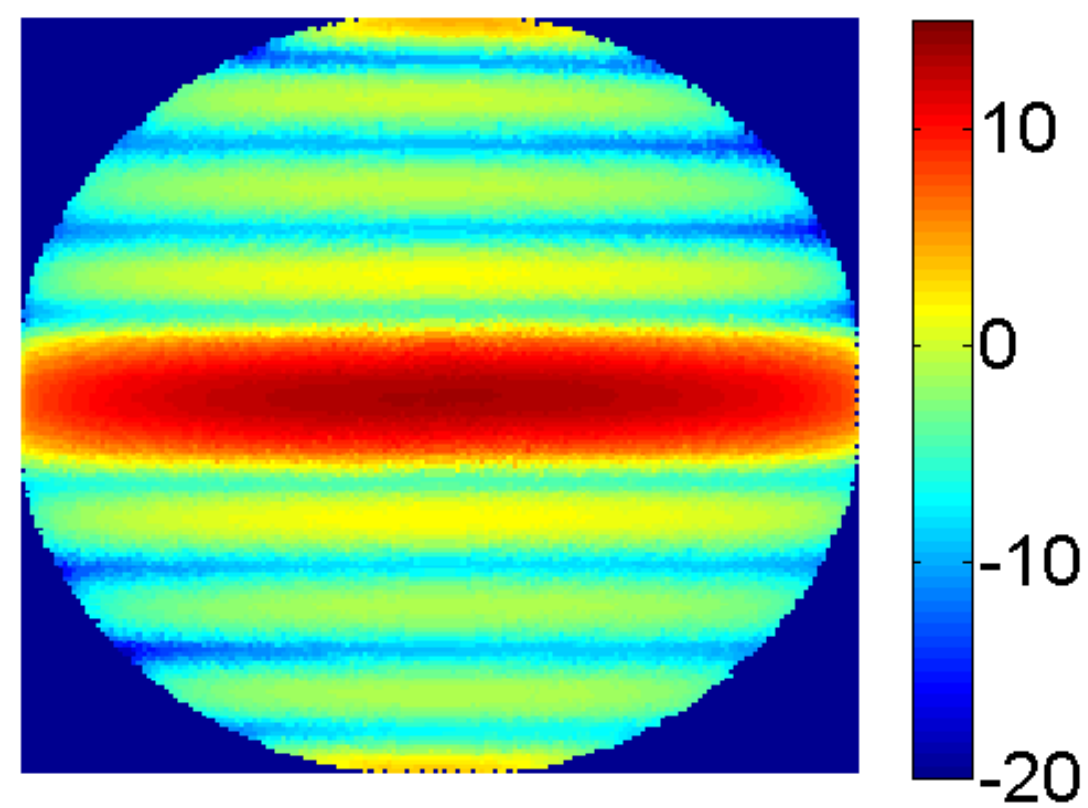

Figure 76 : 10 GHz Linear Array CoPol Gain (dB)
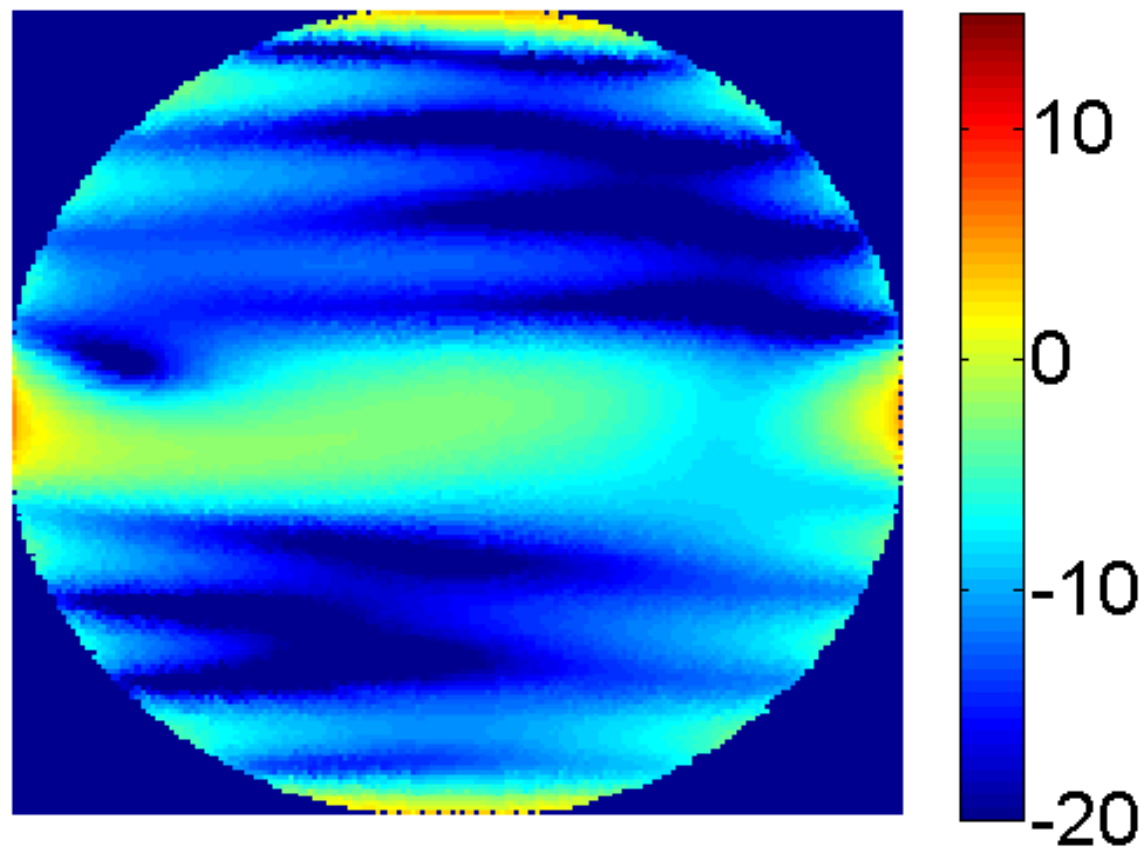

Figure 77 : 10 GHz Linear Array XPol Gain (dB) 
Calculated S-parameter and radiation performance summaries at $10 \mathrm{GHz}$ are captured in Table 8, where all design goals are seen to have been achieved.

\begin{tabular}{|c|c|c|c|}
\hline & Port 1 & Port 2 & Goals \\
\hline Return Loss & $0.054(-25.2 \mathrm{~dB})$ & $0.057(-24.9 \mathrm{~dB})$ & $0.01(-20 \mathrm{~dB})$ \\
\hline P $_{\text {Load }}$ & $13 \%$ & $13 \%$ & $8 \%$ \\
\hline Co-Pol Gain & $12.81 \mathrm{~dB}$ & $12.82 \mathrm{~dB}$ & N/A \\
\hline Cross-Pol Gain & $-3.13 \mathrm{~dB}$ & $-3.31 \mathrm{~dB}$ & N/A \\
\hline Axial Ratio & $2.73 \mathrm{~dB}$ & $2.76 \mathrm{~dB}$ & $3 \mathrm{~dB}$ \\
\hline $\boldsymbol{\eta}$ & $76.4 \%$ & $76.6 \%$ & $75 \%$ \\
\hline
\end{tabular}

Table 8 : Linear Array Simulated S-Parameter and Gain Data

Slight discrepancies from the anticipated power terminated in the load and the axial ratio can be understood in terms of a slight perturbation of the source fields to the X-slot, caused by the slow wave structure. Ideally, the effect of the phase shifter on the waveguide fields would be localized to the three irises. However, it was seen in this case to extend somewhat beyond the immediate iris region and to alter the $\mathrm{X}$-slot unit cell axial ratio and $\mathrm{S}_{21}$ performance. The significance of this phenomenon has not been thoroughly analyzed; however in other design examples not presented in this work, the interaction has effected changes in the linear array performance of similar magnitude. The array design methodology remains verified, via the presence of simultaneous dual-CP radiation with co-located RHCP and LHCP beams at broadside.

\subsubsection{The HFSS Array Performance from 9.5-10.5 GHz}

To determine the bandwidth of the array, the aforementioned discrete sweep was performed in HFSS. The S-parameters and radiation patterns were calculated every $100 \mathrm{MHz}$, using the FEM tetrahedral mesh generated in the adaptive 
solution at $10 \mathrm{GHz}$. This technique is not as accurate as performing adaptive solutions for every frequency point, but generally provides good enough data to demonstrate device performance trends.

The co-polarized gain of the array across the simulated band is captured in Figure 78. The curve indicates the $\mathrm{CP}$ gain of the array ( $\mathrm{dBic})$ at zenith. If the array bandwidth were defined as the span of frequencies over which the gain at zenith was within $1 \mathrm{~dB}$ of the maximum value, then a $4.2 \%$ operating bandwidth was calculated. This definition was employed somewhat arbitrarily and, as with all antennas, the true operating bandwidth must be defined within the context of system requirements.

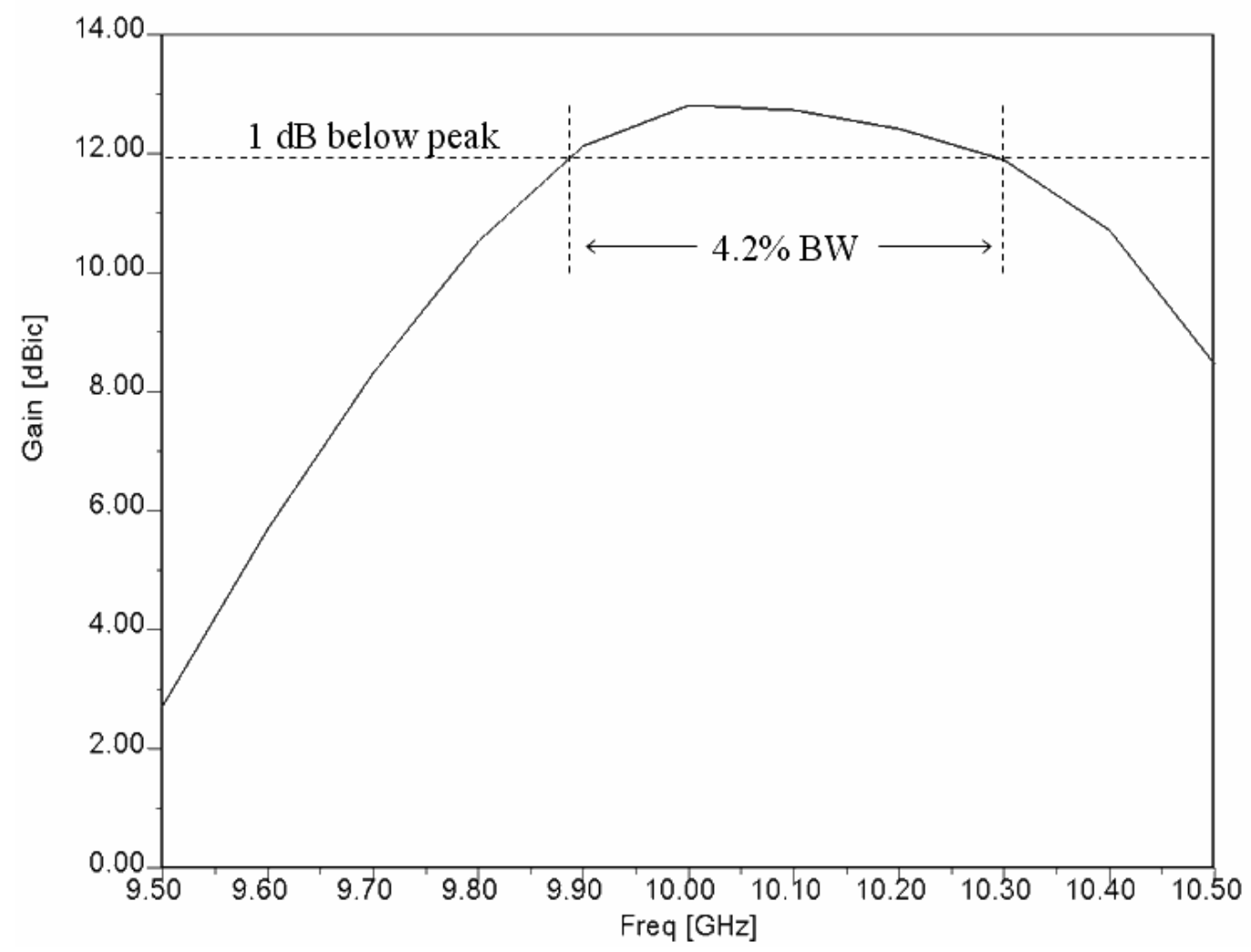

Figure 78 : Linear Array Gain at Zenith vs. Frequency

The next steps required determining the source of the gain roll-off with frequency. The two anticipated sources of performance degradation were the load termination power and the beam squint. These were considered in turn. 
The return loss and power terminated in the load across the band are captured in Figure 79. The return loss remained very good across the band, while the amount of energy terminated in the load became prohibitively large for good aperture efficiency below $9.9 \mathrm{GHz}$ and above $10.4 \mathrm{GHz}$. This was expected, considering the narrow range over which the $\mathrm{X}$-slot unit cell $\mathrm{S}_{21}$ remained close to the design value of 0.78 .

The gain curve appeared to be the approximate inverse of the $\mathrm{P}_{\text {load }}$ curve, leading to the conclusion that the amount of energy terminated in the load (i.e. the varying $\mathrm{X}$-slot unit cell $\mathrm{S}_{21}$ values) is an important limiting factor in array performance.

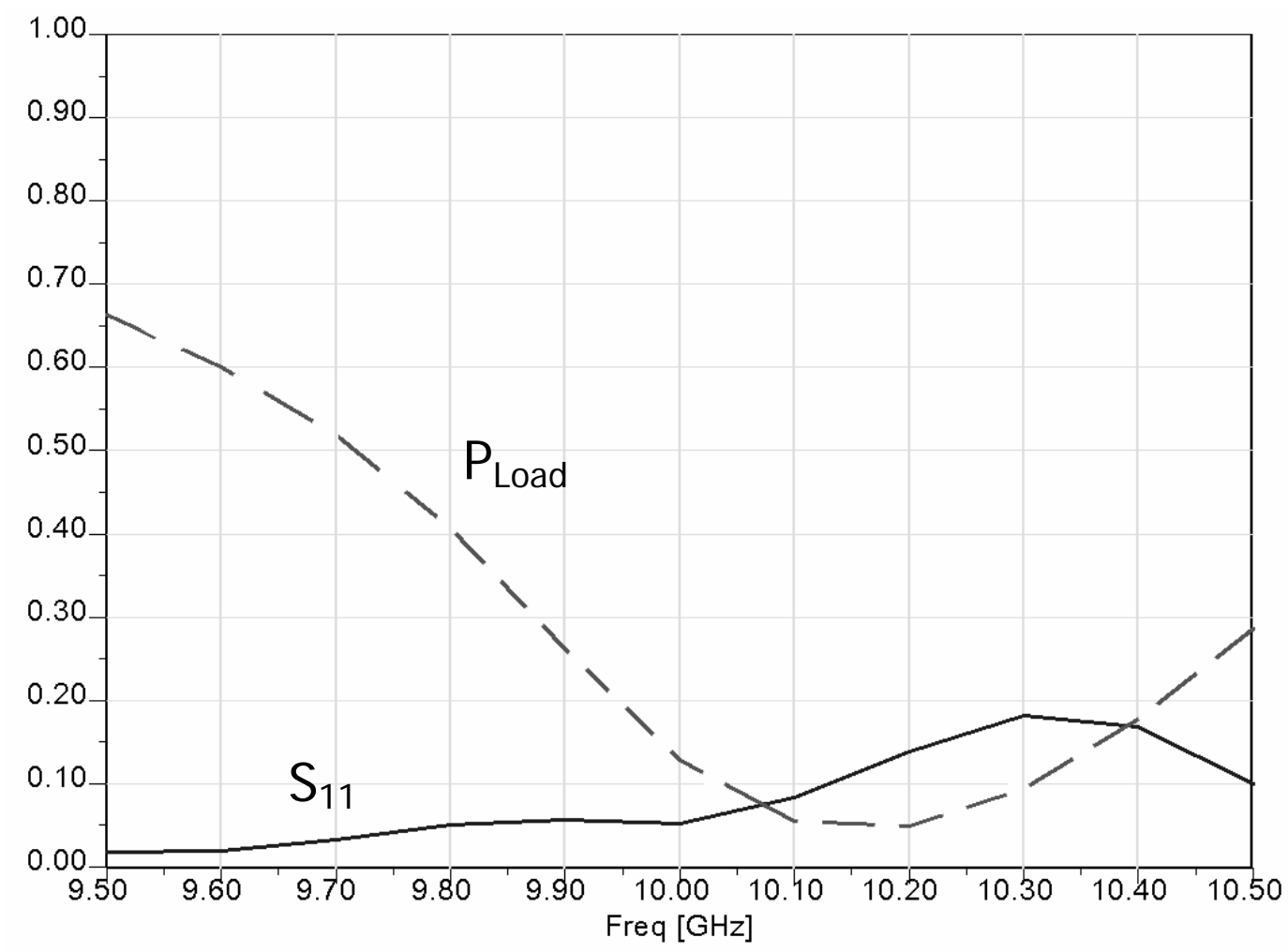

Figure 79 : Linear Array $S_{11}$ and $P_{L o a d}$

Next, the beam squint gain reduction was calculated across frequency. The direction of the main beam is captured in Figure 80. The main beam shifts with frequency because the inter-element phase shift is only zero at the design frequency. As discussed in Chapter 4, the slow wave structure would require superluminal group velocity in order to maintain zero inter-element phase shift 
across a band of frequencies. Attempts were made to off-set the changing phase response of the capacitive slow wave structure with that of an inductive fast wave structure, but nothing successful was achieved. Hemispherical radiation patterns at 9.5 and $10.5 \mathrm{GHz}$ were generated to demonstrate the main beam squint, as seen in Figure 81 and Figure 82.

The impact of the beam squint is clearly present in the five-element linear array. This effect would worsen if the array included more elements, thereby decreasing the beam width in the plane of squint. If the impact of beam squint is unacceptable for a given application, a sub-arrayed approach could be considered where a long linear array is broken into a series of corporate-fed four or five element sub-arrays. The net beam squint should be reduced [6-1].

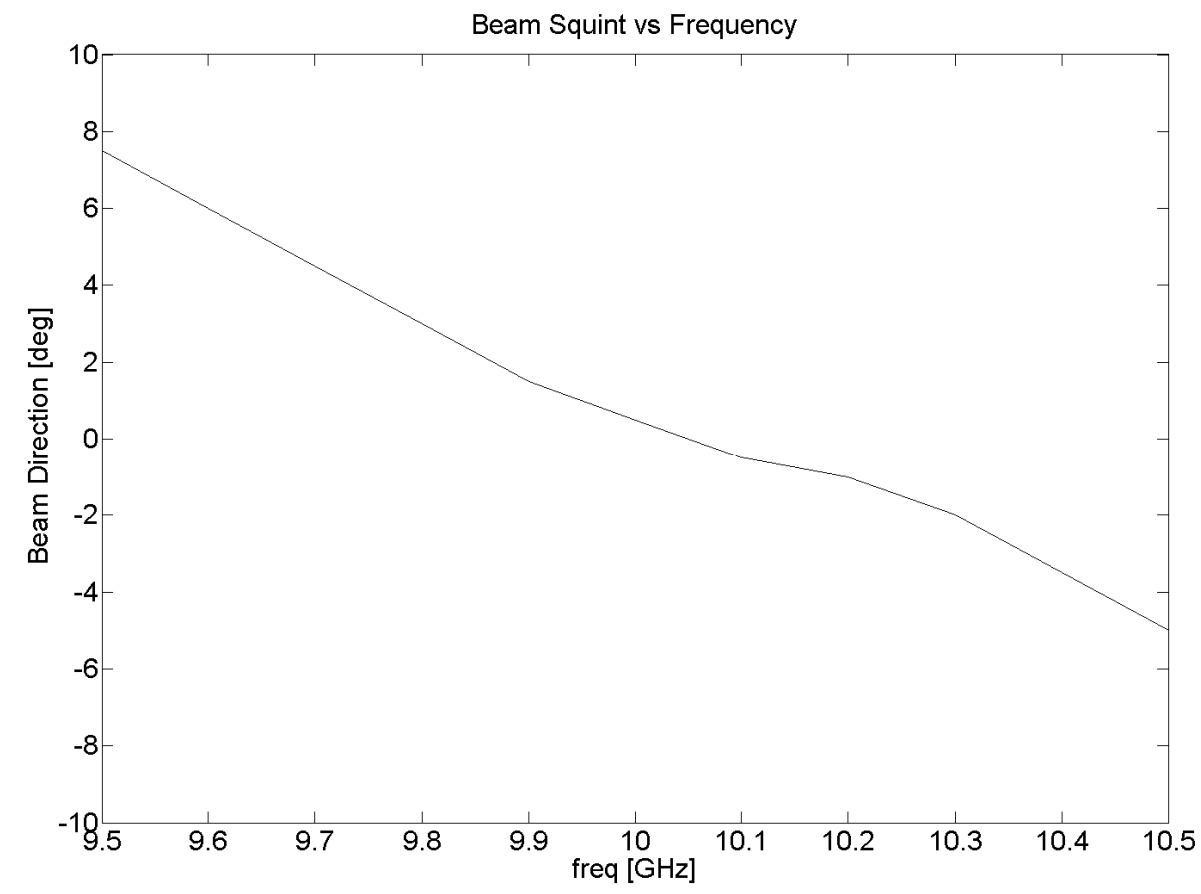

Figure 80 : Linear Array Main Beam Direction vs. Frequency 

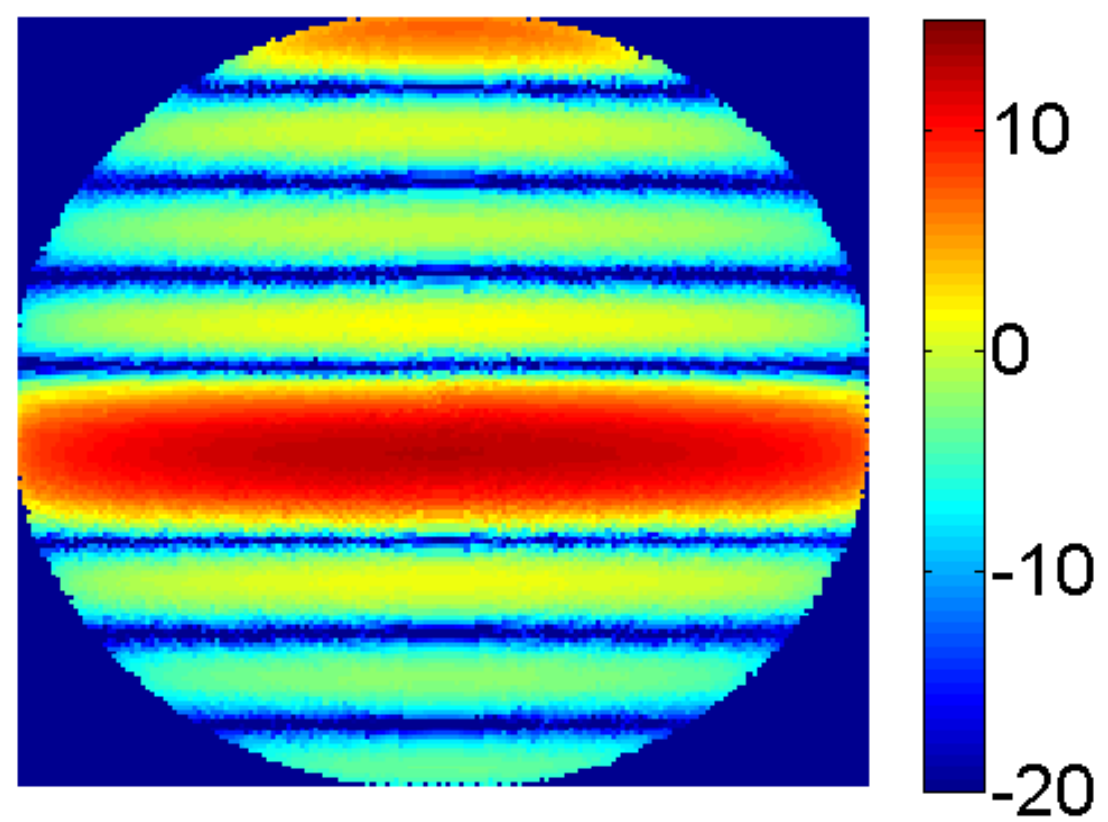

Figure 81 : 9.5 GHz Linear Array CoPol Gain (dB)

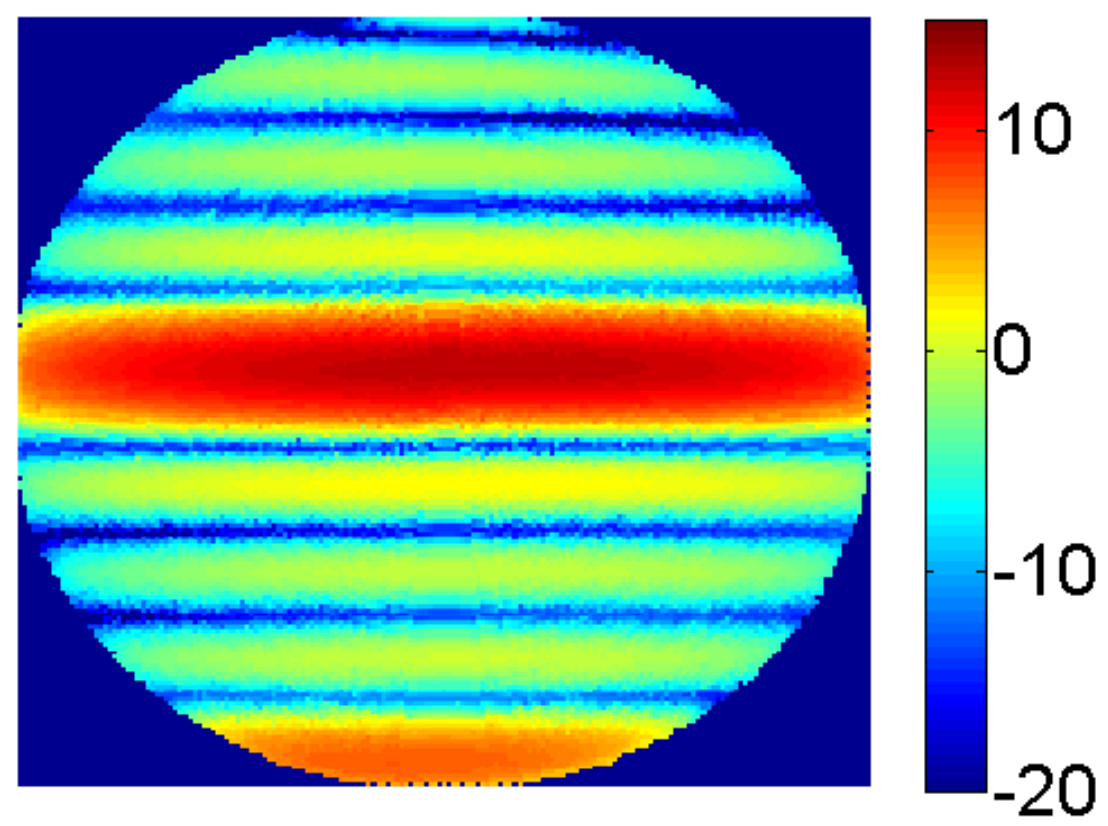

Figure 82 : 10.5 GHz Linear Array CoPol Gain (dB) 
Finally, the assumption was made that for some applications, the LHCP and RHCP beams could be allowed to diverge. Furthermore, it was assumed that in these situations, mechanical steering could exist, permitting the array to be physically pointed for maximum gain in the direction of the beam, thereby reducing the impact of beam squint. While the impetus for much of this work assumed this was not acceptable, it was also recognized that in traditional $\mathrm{CP}$ waveguide slot architectures, the RHCP and LHCP beams diverge by $80^{\circ}-100^{\circ}$, making the mechanical positioning requirements much more severe than in this case where each main beam overlaps, despite not sharing the same volume. Making this assumption, then, the gain of the array in the main beam direction is captured in Figure 83. Employing the previous definition of bandwidth, the array performance would extend from $4.2 \%$ up to $5.5 \%$. The increase is slight, but noteworthy.

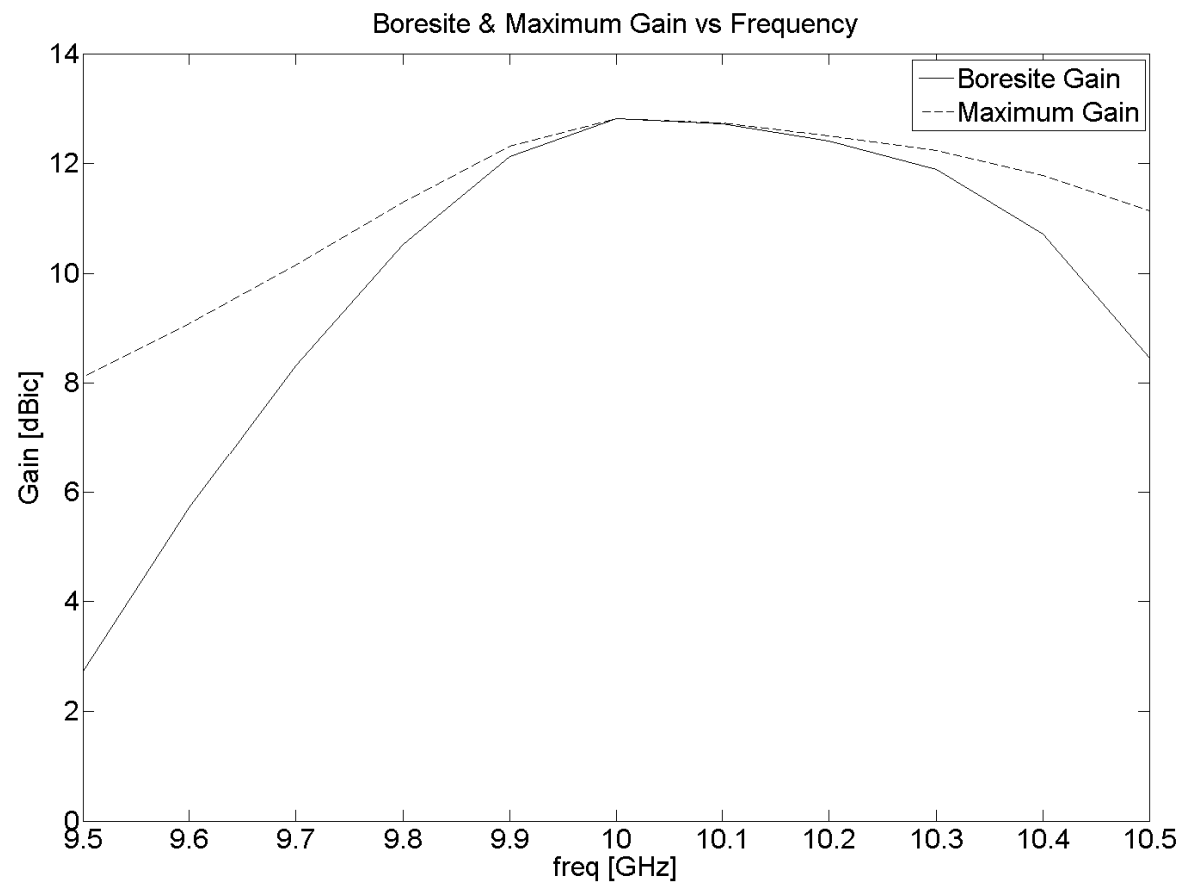

Figure 83 : Linear Array Boresite and Peak Gain vs. Frequency 


\subsubsection{The CST Comparison Model}

To compare the results of the HFSS model, a nearly identical model was constructed in CST. Due to slight modeling protocol differences between the pieces of software, an identical model was not possible in CST. The differences were subtle, for example, the linear array could not radiate into an infinite halfspace, but instead had to radiate into a full space with an electrically large ground plane. The impact of these differences was slight and should be evident in the simulated data.

To exhibit the similarity of the CST simulation, screen images from the model are shown in Figure 84.
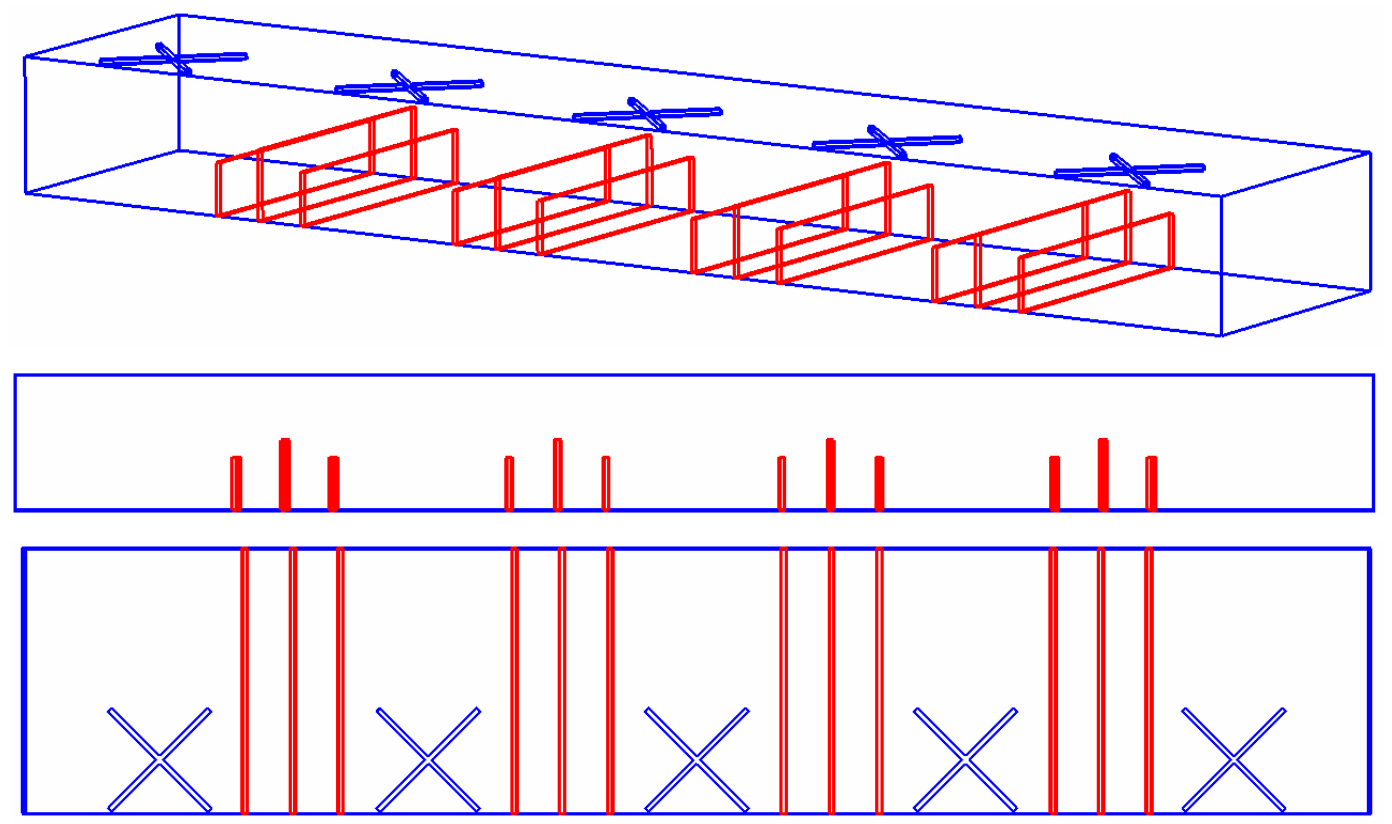

Figure 84 : CST Linear Array Design Example Model

\subsubsection{CST vs. HFSS : Radiation Patterns}

The first point of comparison between CST and HFSS was in the radiation patterns at $10 \mathrm{GHz}$. The co-polarized directivity patterns of both are shown in Figure 85. For reasons unknown, CST does not provide the tools to plot gain of 
an antenna (i.e., taking into account all system losses), thus the directivity of each solution had to be compared.

Despite employing very different solution techniques (FDTD vs. FEM), the calculated linear array directivity patterns are remarkably similar in HFSS and CST. This was especially observed throughout the main beam region, as seen in Figure 86, an expanded view of the main lobe. The divergence between the solutions was a result of about a $0.25^{\circ}$ shift in the beam direction.

To further explore the issue of discrepancies in the radiation pattern in the side lobe regions, calculations were made over the entire hemisphere at 9.5, 10, and $10.5 \mathrm{GHz}$. This data is captured in Figure 87, Figure 88, and Figure 89. Comparing the HFSS and CST full pattern data, a good degree of similarity was observed. The basic structure of each radiation pattern was the same - a main beam at broadside with zero grating lobes. Additionally, the direction of beam squint with shifts in frequency was identical in both solution sets. An odd ring pattern appeared in each of the CST plots near the horizon, which made little physical sense. It was concluded the HFSS patterns were probably more accurate because they simply looked more realistic. The HFSS help file provides the equations employed in calculating the far field pattern, but the technique is hidden from the CST user, so further investigation of the discrepancy could not be pursued.

Ultimately, the observed differences beyond the main beam region are curious, but of little consequence for two reasons. First, the array was designed for maximum aperture efficiency and, therefore, will have relatively high side lobes - that is, the side lobe levels are not a primary concern. Second, side lobe level discrepancies are anticipated due to the differences between the HFSS and CST ground plane implementations. In HFSS, edge diffraction is not included because the radiated fields are terminated over an infinite ground. In CST, a step exists at the edge of the electrically large ground plane (around $5 \lambda$ per side), which will cause diffraction effects to be seen in the pattern. 


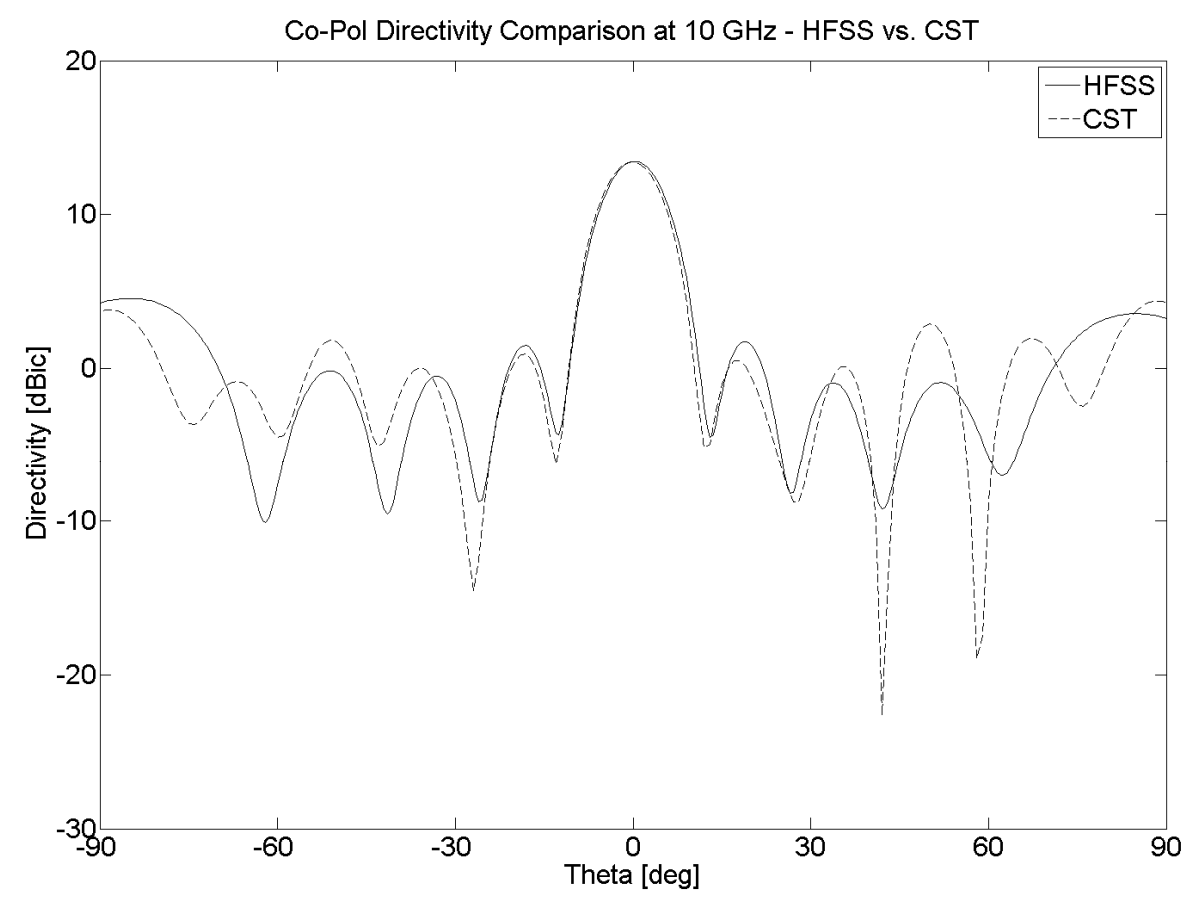

Figure 85 : Linear Array 10 GHz Directivity, HFSS vs. CST

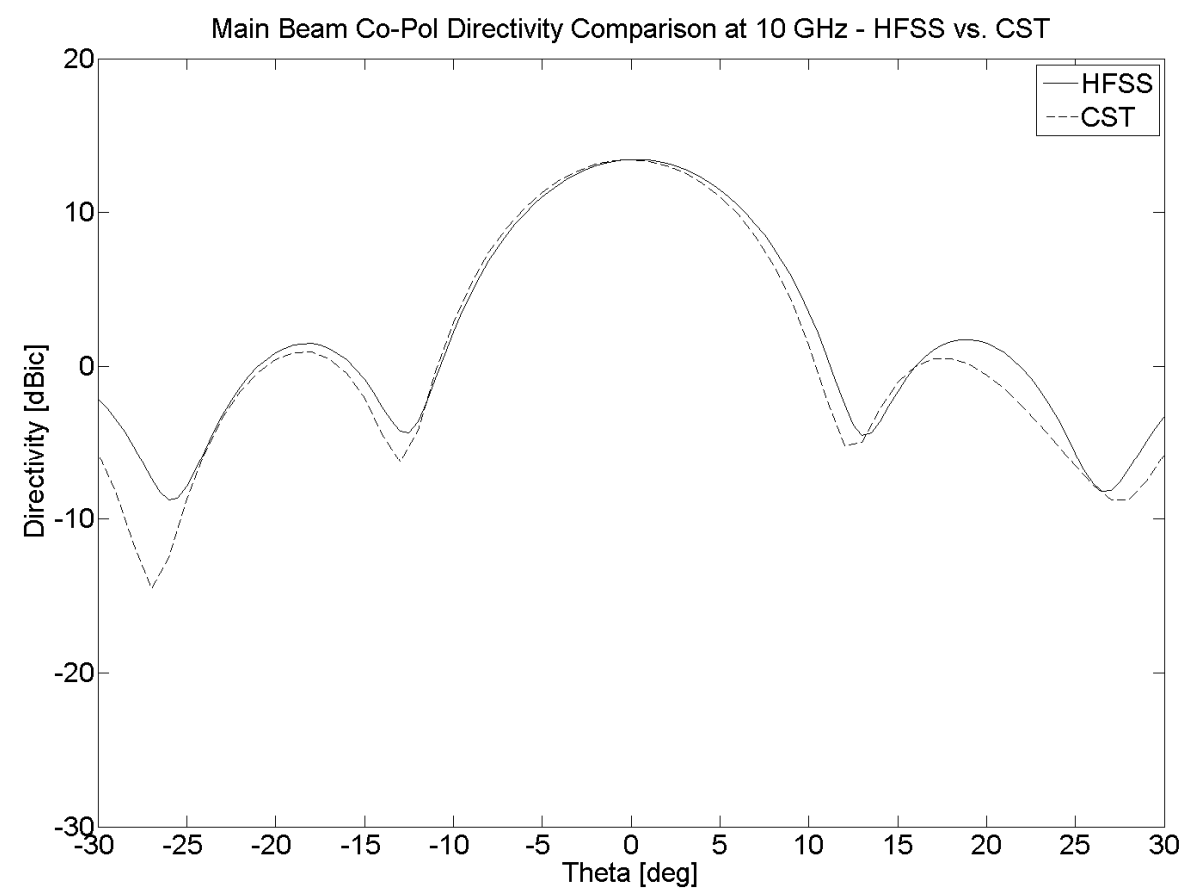

Figure 86 : Linear Array $10 \mathrm{GHz}$ Main Beam Comparison, HFSS vs. CST 


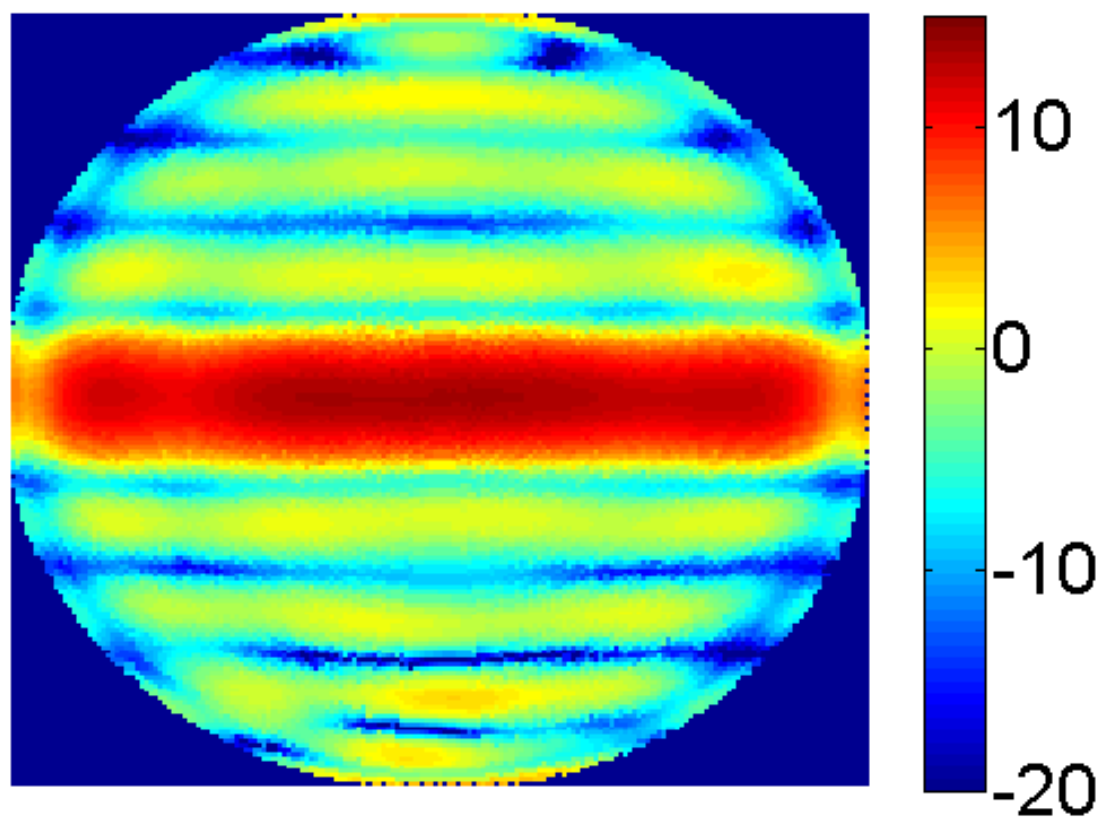

Figure 87 : CST Linear Array 10 GHz CoPol Directivity (dB)
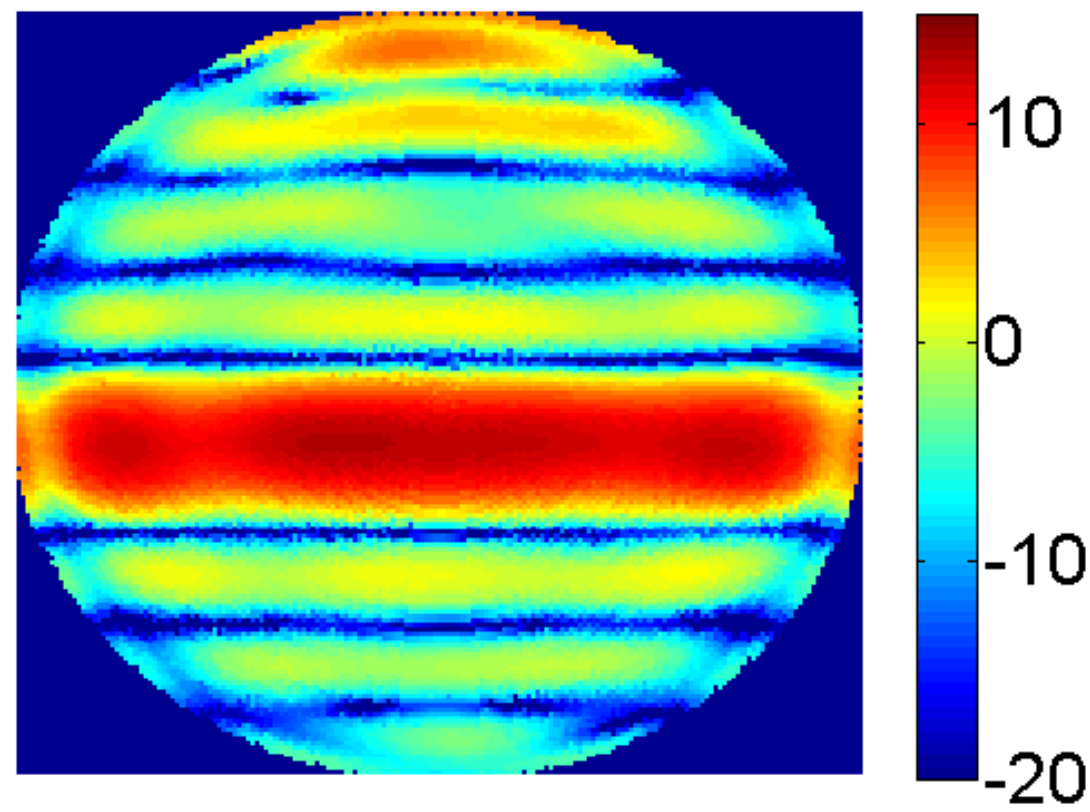

Figure 88 : CST Linear Array 9.5 GHz CoPol Directivity (dB) 

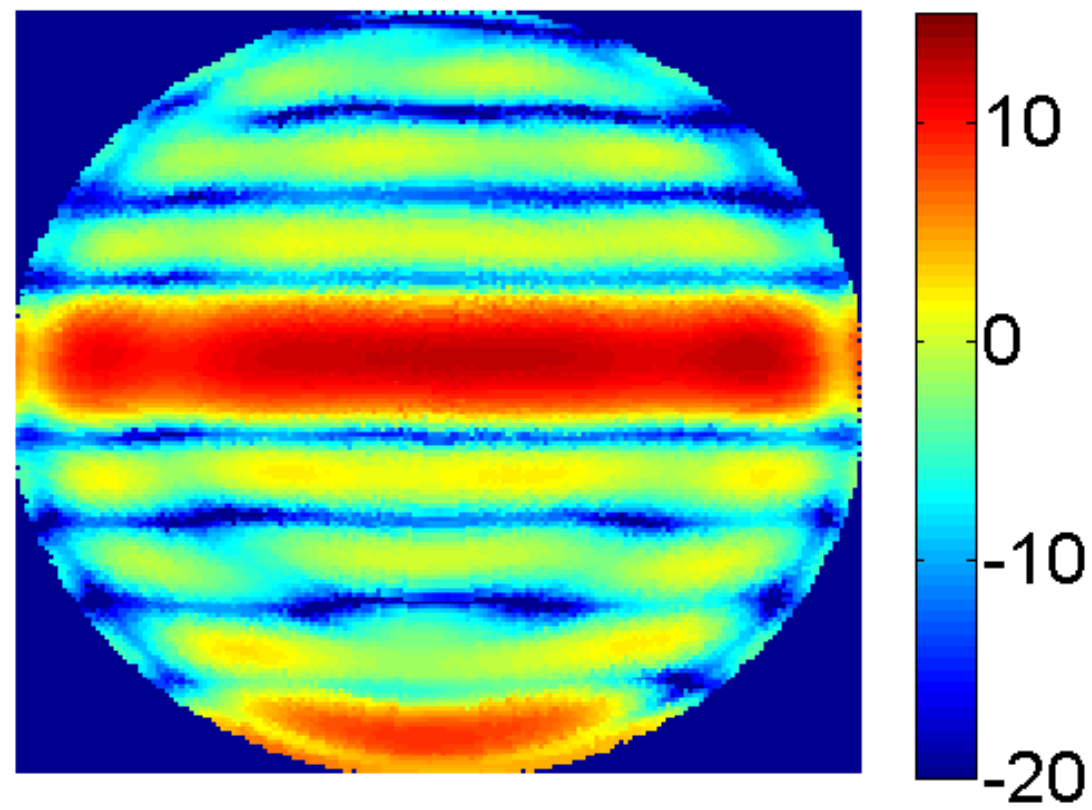

Figure 89 : CST Linear Array 10.5 GHz CoPol Directivity (dB)

Despite the slight differences observed between the HFSS and CST calculated radiation patterns, they are similar enough to strongly confirm the linear array gain performance, in lieu of experimental data.

\subsubsection{CST vs. HFSS : $P_{\text {Load }}$ and $S_{11}$}

The second point of comparison between HFSS and CST was the return loss and power terminated in the load. Because the CST solution was in the time-domain, all the frequency response data was generated simultaneously and was not a concatenation of multiple narrow-frequency response solutions, like in HFSS. The $\mathrm{S}_{11}$ data from both simulators is shown in Figure 90. As with the radiation pattern information, remarkably good agreement was observed over the majority of the data set, offering confirmation of the simulations. 


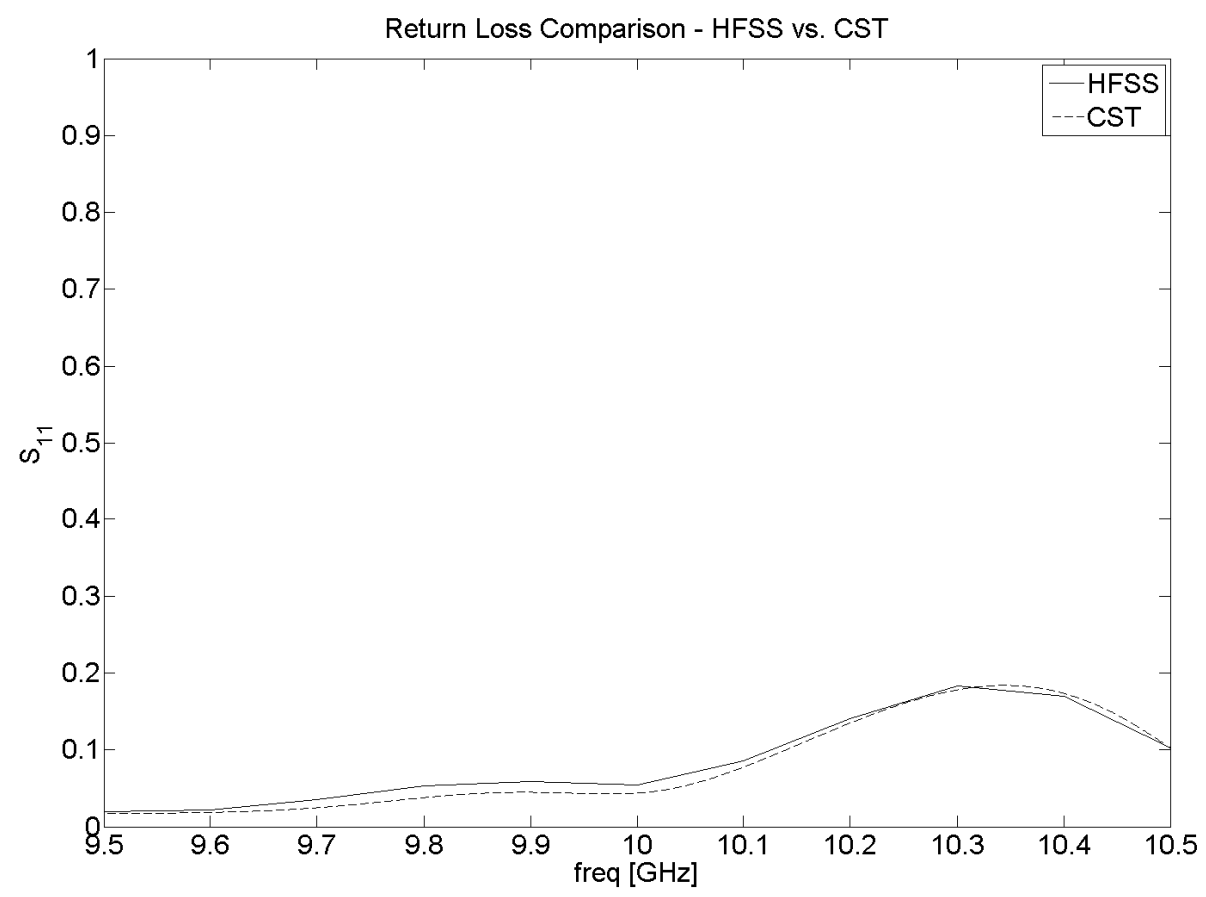

Figure 90 : Linear Array $S_{11}$ HFSS vs. CST

The power terminated in the load for both HFSS and CST is seen in Figure 91. While both plots exhibit very similar trends, a curious divergence was observed from 9.5 to $10.1 \mathrm{GHz}$. For reasons unclear, the two solutions diverge in that region. Several attempts were made to solve this discrepancy; including improving the mesh densities in both solvers, but it could not be removed. Overall, the two trends exhibited sufficiently similar results to provide good confidence in the simulation. When this fact was combined with the outstanding agreement in the other plots above, an aggregate very good agreement was found between HFSS and CST. This agreement was viewed as strong evidence for the performance of the array and confirmation of the design methodology. 


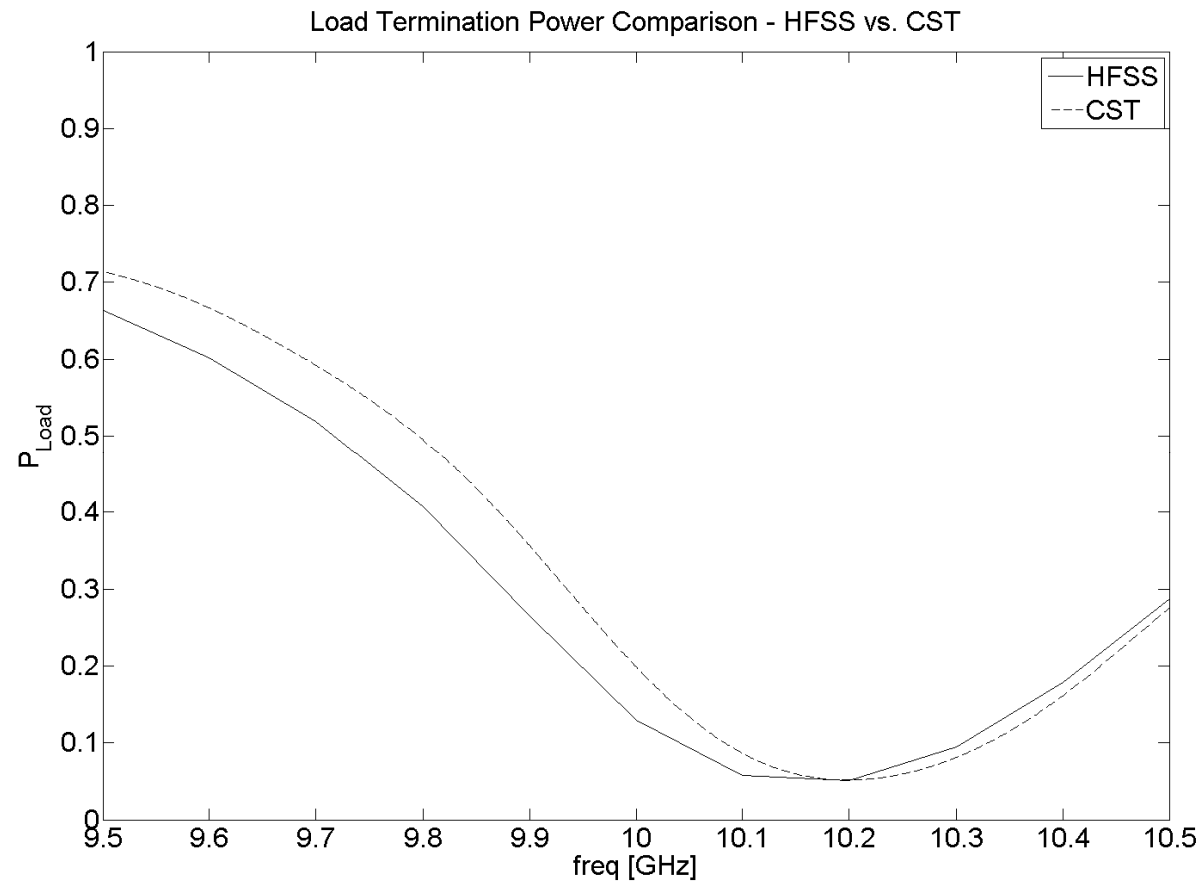

Figure 91 : Linear Array $\mathbf{P}_{\text {load }}$ HFSS vs. CST

\subsection{Array Design Example Conclusions}

A novel array topology and design methodology example has been presented in this chapter. After stating the design steps, each was carried out in as transparent a fashion as reasonably possible. The philosophical difference between the design methodology presented here and standard, transmission line design methods, which depend on equivalent circuit models of slot radiators, for linearly polarized slots, comes in the intended audience. Modern designers rely heavily on ubiquitous software packages — such as HFSS and CST — which employ highly capable algorithms for solving generic antenna problems, but lack the sophistication and optimization of many specialized academic codes. The aim of the design process in this work was to cater to the needs and capacity of the majority of designers that could benefit from the proposed array topology.

Through the design example in this chapter, the relative simplicity of each step in the design procedure was revealed. Any designer with moderate 
familiarity of one of the referenced pieces of commercial software (or an equivalent) should be capable of carrying out all the design steps within a few hours. The optimization process employed in the slow wave structure is the only potential bottleneck in the design steps. If each cost function evaluation required 5-10 minutes because one of the generic software packages were employed, then the fourth step in the design process could require up to a full day, depending on the solution convergence rate. The final step of a full array simulation is optional and a function of the computing resources available to the designer. A wellknown shortcoming of generic software packages is their inefficient, brute-force approach to problem solving. If a linear array with $10+$ slot elements or, perhaps, a full planar array, were the design goal, the final simulation step could be very challenging even with modern top-of-the-line workstations.

The primary performance goals of the proposed array topology were observed in the HFSS gain patterns and S-parameter curves. The main beam of the linear array was focused in the broadside direction, with good return loss and high aperture efficiency. As expected, the beam squinted with frequency and the unit cell $\mathrm{S}_{21}$ value did not remain constant, limiting the array performance bandwidth.

Linear array performance in both radiation patterns and S-parameter data were confirmed via simulations in CST. Exceptional agreement was observed throughout the main beam region of the array, with the only observable differences being a $0.25^{\circ}$ shift in beam direction. If the simulation carried out were an array with something akin to a corporate feed, where all elements should be in phase by virtue of the feed structure employing uniform current paths, then this level of agreement between two philosophically orthogonal simulation tools would be quite good. Since, however, the main beam direction in the design example was artificially produced at broadside via the slow wave structure passive phase shifter effects and would not be expected by default, this level of agreement in the main beam direction is considered exceptional. It implies that the performance of the slot antennas, the slow wave structures, and all associated interactions were all modeled equally well in both tools. 
Exceptional agreement was also observed between the HFSS and CST return loss data across the 9.5 to $10.5 \mathrm{GHz}$ band. The calculated power terminated in a matched load agreed well for a portion of the band, but diverged slightly in the 9.5 to $10.1 \mathrm{GHz}$ region. The reason for this divergence has not been discovered, but is considered of little consequence as the curves exhibited the same trends. 


\section{Chapter 7: Conclusions and Future Work}

\subsection{Overview and Conclusions}

Two primary goals were set for this work. The first goal was to develop a novel array topology intended to produce dual CP radiation from a waveguide slot array where the LHCP and RHCP beams are co-located at zenith. The antenna topology was anticipated to address systems which simultaneously demand low feed loss, high aperture efficiency, and dual CP operation. The second goal was to develop a robust, generic design procedure for this array.

A common fault of academic engineering work is a limited range of applicability and accessibility to those outside of academia. As discussed previously, the history of waveguide slot radiator research had a strong beginning in this regard. Design tools were reported that were universally accessible - e.g., design curves and simple equations. As time progressed and digital computers came into vogue, however, the field of waveguide slot radiator research changed directions. Specialized MoM codes were developed which simultaneously increased the accuracy of the design techniques (e.g., mutual coupling could be more precisely considered) and raised barriers to entry for the non-researcher. However, because the powerful analysis tools were not made publicly available, the results, while making for very interesting and impressive publications, were of little use to their intended audience!

A primary philosophical intention of this work has been to overcome these historical limitations of academic waveguide slot radiator research. Commercial software was employed with the belief that if generic design methods were developed for generic tools, the array topology should be within the reach of any industrial engineer using the same type of generic tools. Moreover, the reach of the work could be as widespread as these seemingly ubiquitous tools (HFSS, CST, etc.).

To conclude this work, a brief overview of each chapter is presented. 


\section{Chapter 1}

The beginning of this work introduced an historical context via a brief overview of the major developments in waveguide slot radiator research since its inception in the 1940 s by Watson.

\section{Chapter 2}

The second chapter outlined some of the specific challenges associated with dual circularly polarized waveguide slot radiators arrays and proposed a notional solution including a passive phase shifter and a CP slot element.

\section{Chapter 3}

The third chapter analyzed several potential radiators and passive phase shifters against the demands of the proposed array architecture. Simulation data for down selection criteria was presented for the radiators, with the X-slot ultimately chosen due to the T-Slot and OCSP both exhibiting tilted-beam array factors. Dielectric loading and slow wave structures were compared for the passive phase shifter. The discrete slow wave structure was chosen because it had the lowest loss and was the simplest to manufacture. Finally, a proposed design procedure was outlined.

\section{Chapter 4}

A design methodology for the discrete slow wave structure passive phase shifter was developed in the fourth chapter. Analytical expressions from Marcuvitz and Collin were reviewed as a starting point in the design process. It was observed that with a large enough number of irises per unit cell, the required guided wave number could be predicted with reasonable accuracy; however, the return loss was generally poor at the design frequency. The analytical solution was then

employed as a starting point for an optimization algorithm driven by a commercial software package highly specialized for waveguide structures. It was observed that with the proper selection of design constraints, a discrete slow wave 
structure which simultaneously satisfies the wave number and return loss design criteria could be achieved in a short amount of time.

\section{Chapter 5}

The X-slot radiator was the focal point of the fifth chapter. The practical usefulness of an historical design equation presented by Simmons was reconsidered. For the majority of design scenarios, where an X-slot with leg lengths on the order of $\lambda / 2$ is required, the design equation was seen to be insufficient because it demanded an impractical slot offset. Since Simmons' equation is the only design information available in the open literature, a parametric study of the design variables for an X-slot was conducted. The results of which culminated in a set of generic design guidelines predicated on one of the previously mentioned pieces of commercial software being utilized by the designer.

\section{Chapter 6}

The final technical chapter of the work presented a transparent start-to-finish design example. Each step was presented in as open a fashion as possible to increase the repeatability by future readers of this work. A five element X-slot array was simulated in HFSS. The calculated results demonstrated the proposed array topology did in fact address the previously discussed design problems for dual $\mathrm{CP}$ arrays. In a structure that would be fairly simple to manufacture and can be easily designed with off-the-shelf software, dual CP radiation was observed with the RHCP and LCHP beams co-located at zenith. Return loss lower than -20 $\mathrm{dB}$ and aperture efficiencies over $75 \%$ were calculated. To confirm the array operation, an identical structure was simulated in CST, which employs the FDTD method. The CST S-parameter and radiation patterns were observed to be in very good agreement with the HFSS results, further strengthening the validity of the proposed array topology. 


\subsection{Future Work}

A few parting remarks related to future work are in order.

1. Construction of linear and planar arrays based on the presented design methodology needs to be undertaken to further reinforce the method.

2. One of the main goals in the presented design approach was to maximize aperture efficiency and gain. As was seen earlier, this step greatly simplifies the radiating element design process because each X-slot in the array is identical. Further study should be conducted to determine sufficient design techniques for dual CP low side lobe arrays. That is, the possibility of achieving classic amplitude tapers (Tayor, Chebyshev, etc.) within the context of a traveling wave array fed from both ends must be examined.

3. The impact of mutual coupling was also largely ignored in the present work. In the context of high gain traveling wave arrays where side lobe levels are somewhat irrelevant, mutual coupling is traditionally ignored. When very low side lobe levels are a critical design parameter, inter-element mutual coupling must be considered.

4. Finally, the perturbation of unit cell X-slot performance by the slow wave structure passive phase shifters requires further analysis. As with mutual coupling, this topic was largely ignored because it was not seen to shift the unit cell radiation and S-parameter performance by a significant amount. In the context of an array with much more stringent design goals, however, this perturbation will require consideration. 


\section{Appendix A : An Alternate X-Slot Theory of Operation}

During the X-slot parametric study in Chapter 5, a relationship between slot return loss $\left(\mathrm{S}_{11}\right)$ and axial ratio was repeatedly observed. This relationship is further investigated in this appendix and a new theory of operation is proposed, which leads to an equivalent single-slot design approach. The theory is not developed beyond the range of the simulation data, but is confirmed via simulation of an alternate version of the X-slot - the Double Crescent Slot (DCS).

Simmons proposed a theory of operation which emphasized the complex relationship between the transverse and longitudinal source fields of the feed waveguide dominant mode. As discussed above, he theorized that any slot placed at the proper location would produce good CP radiation. This theory, however, did not explain the relationship between $\mathrm{S}_{11}$ and axial ratio. It is proposed here that the $\mathrm{X}$-slot is better understood as a juxtaposition of two linear, compound slots.

A study of two isolated compound slots was conducted to determine how the complex $S_{11}$ and aperture fields changed with offset. All other parameters were chosen similarly to the X-slot of Chapter 6 .

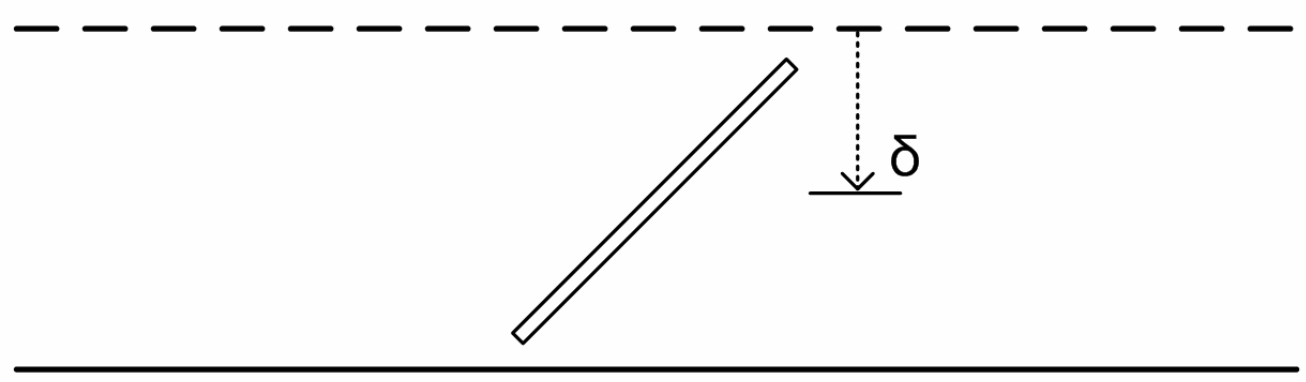

Figure 92 : Compound Slot Study Unit Cell 
It was initially confirmed that the $\mathrm{S}_{11}$ of both X-slots can be determined via the S-parameters from a single unit cell. $S_{22}$ from the unit cell in Figure 92 was observed to be identical to $S_{11}$ from the complementary compound slot which composes the X-slot. Thus, the S-parameter studies could be carried out with a single unit cell. The compound slot was varied from zero offset to as far as possible.

The magnitude of the maximum $\mathrm{S}_{11}$ and the resonant frequency (i.e. the frequency where $S_{11}$ was at a maximum) were confirmed to be identical for both slots. The range of $\mathrm{S}_{11}$, as observed in Figure 93, was much higher than the Xslot $\mathrm{S}_{11}$.

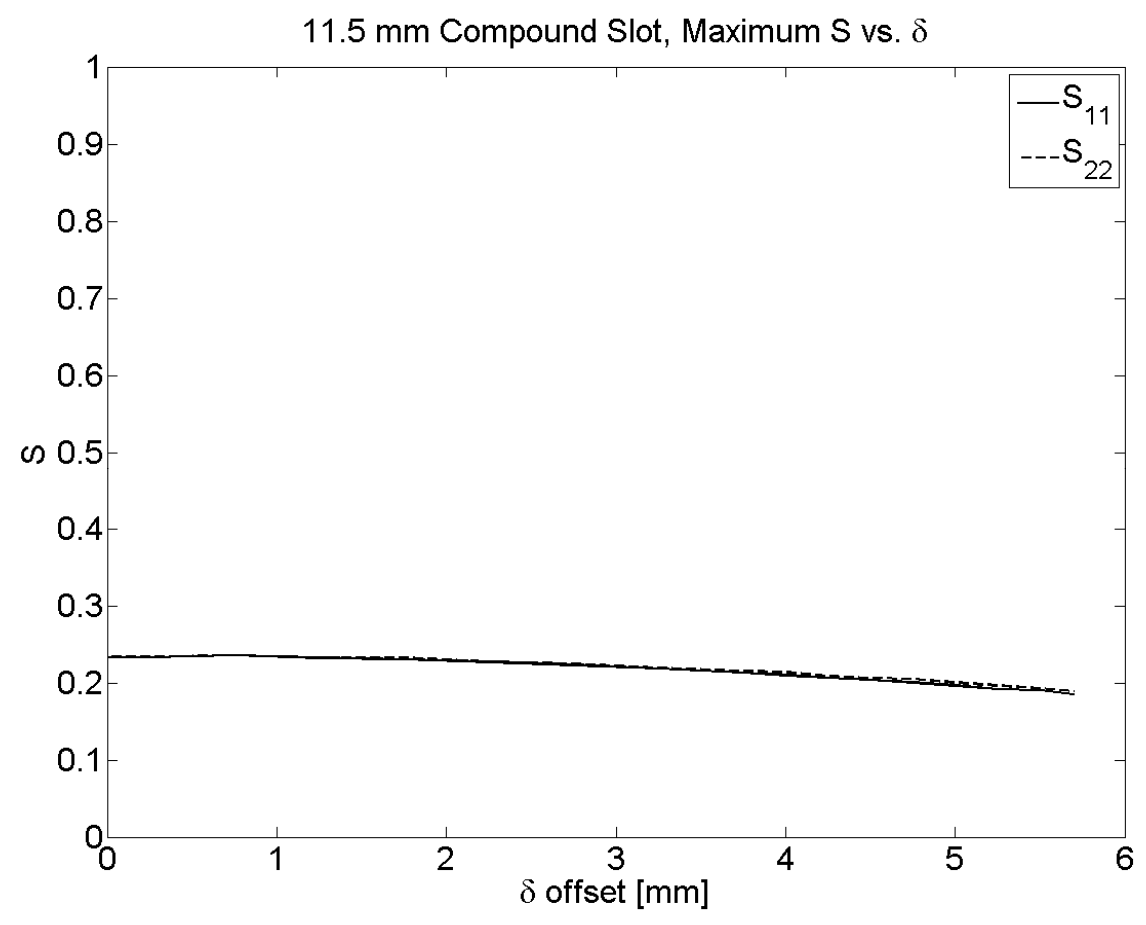

Figure 93 : Compound Slot $S_{11}$ Magnitude vs. $\delta$

Next, the phase of the compound slot $\mathrm{S}_{11}$ was studied, at resonance, against shifts in slot offset. The data in Figure 94 demonstrated that as slot offset increased, the phase of $\mathrm{S}_{11}$ changed - that is, slot reactance increased. As with previous simulations, the phase calibration plane was established close to the center of the slot, to mitigate transmission line effects in the $\mathrm{S}_{11}$ data. 


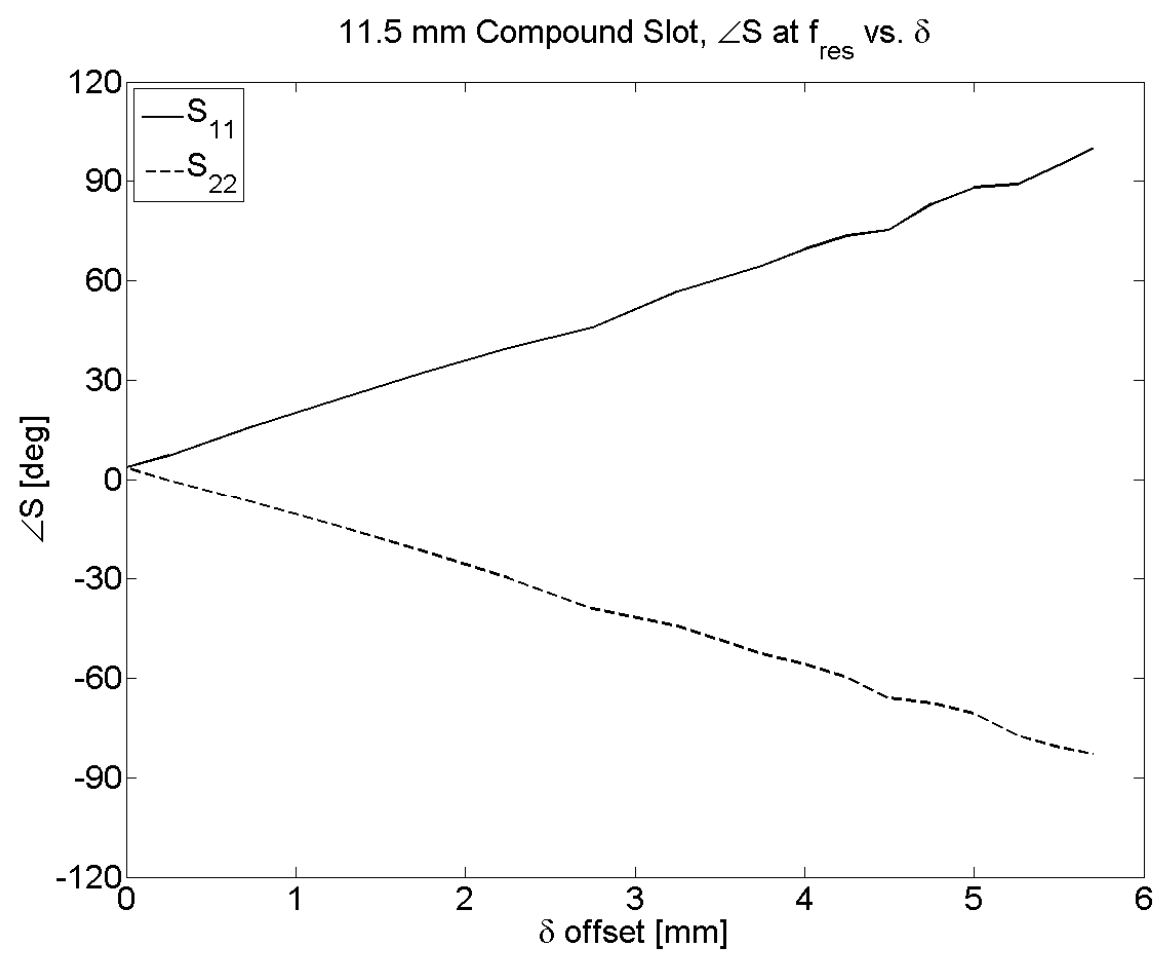

Figure 94 : Compound Slot $S_{11}$ Phase vs. $\delta$

The $\mathrm{S}_{11}$ phase shifted until, at the largest possible offset, the compound slot became purely reactive. The juxtaposition of two compound slots with equal and opposite effective reactances would be expected to simultaneously provide conjugately match each other. To confirm this theory, the real and imaginary part of the sum of $\mathrm{S}_{11}$ from each compound slot was plotted in Figure 95. As anticipated from the simulated phase data, the two compound slots conjugately match each other across the frequency band. For smaller offsets, however, the real component of the reflection coefficient dominates the return loss and the conjugate matching is minimal. As slot offset increases toward a terminal value, the purely reactive reflections cancel, thus providing the superb return loss performance seen throughout the X-slot parametric studies. 


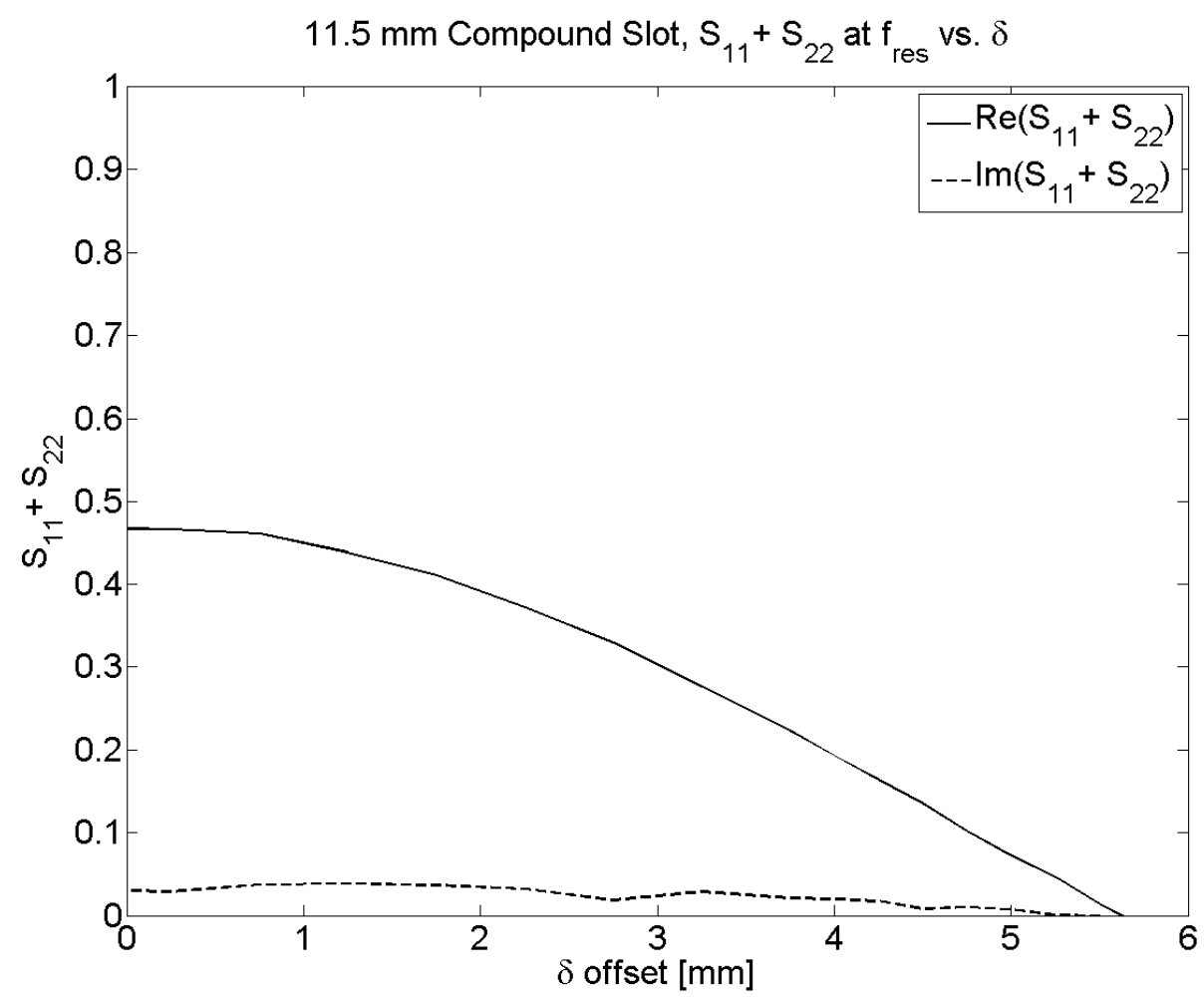

Figure 95 : Compound Slot Conjugate Matching vs. $\delta$

To investigate the axial ratio relationship, the slot aperture fields were examined, at resonance, for each of the two compound slots. Unlike the Sparameter analysis, two unit cells had to be simulated for every offset value. The dominant polarization average aperture field magnitude was calculated for each slot. A plot comparing the relative magnitudes is found in Figure 96. Similarly, the dominant polarization aperture field phases were calculated for each offset value. Relative slot field phases are plotted in Figure 97.

As with $\mathrm{S}_{11}$, the relative slot field magnitudes did not change with offset, but the phase relationship did change. Whereas the $S_{11}$ phases approached a $180^{\circ}$ separation, the aperture field phases approached a $90^{\circ}$ separation. Thus the two conditions for good CP radiation from orthogonal slots were observed. 


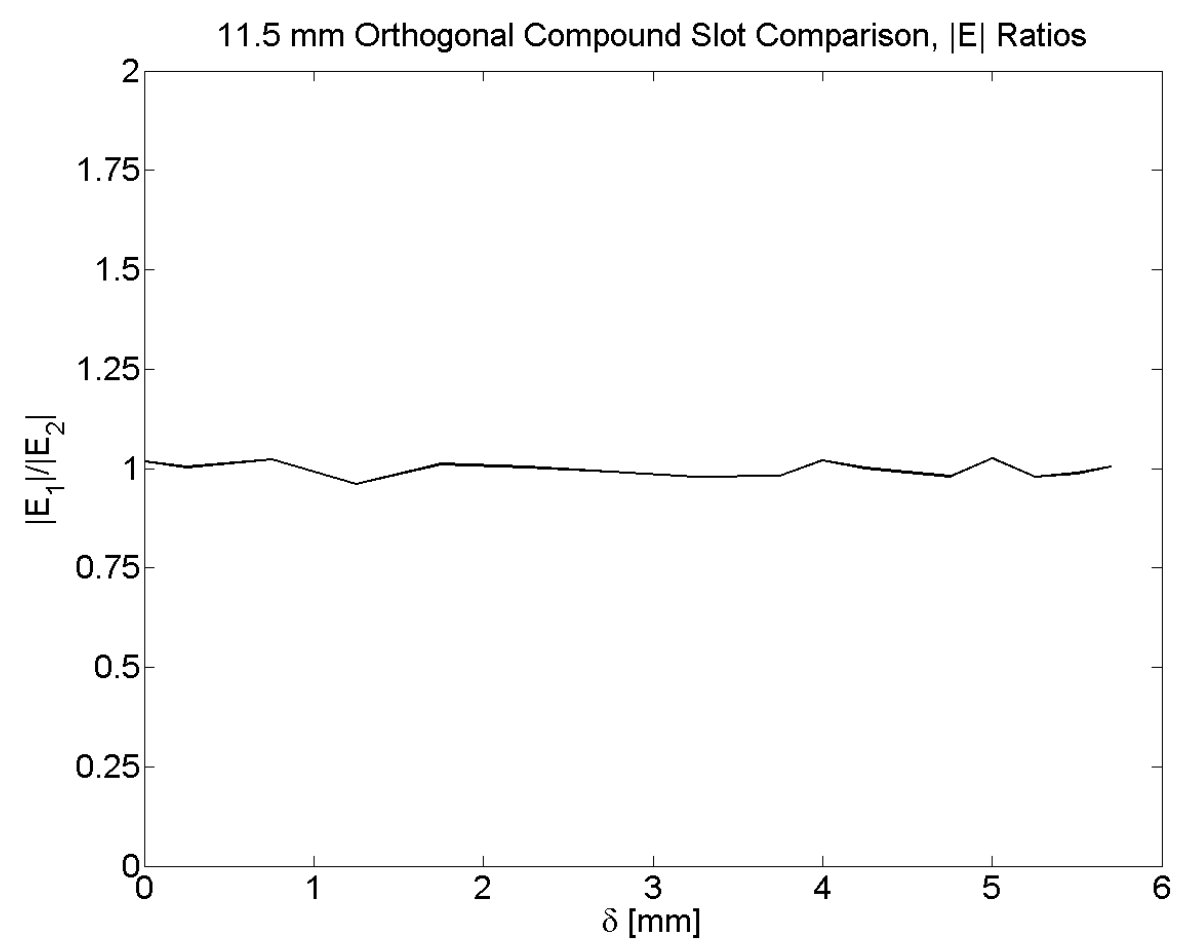

Figure 96 : Compound Slot Aperture Field Magnitude Comparison

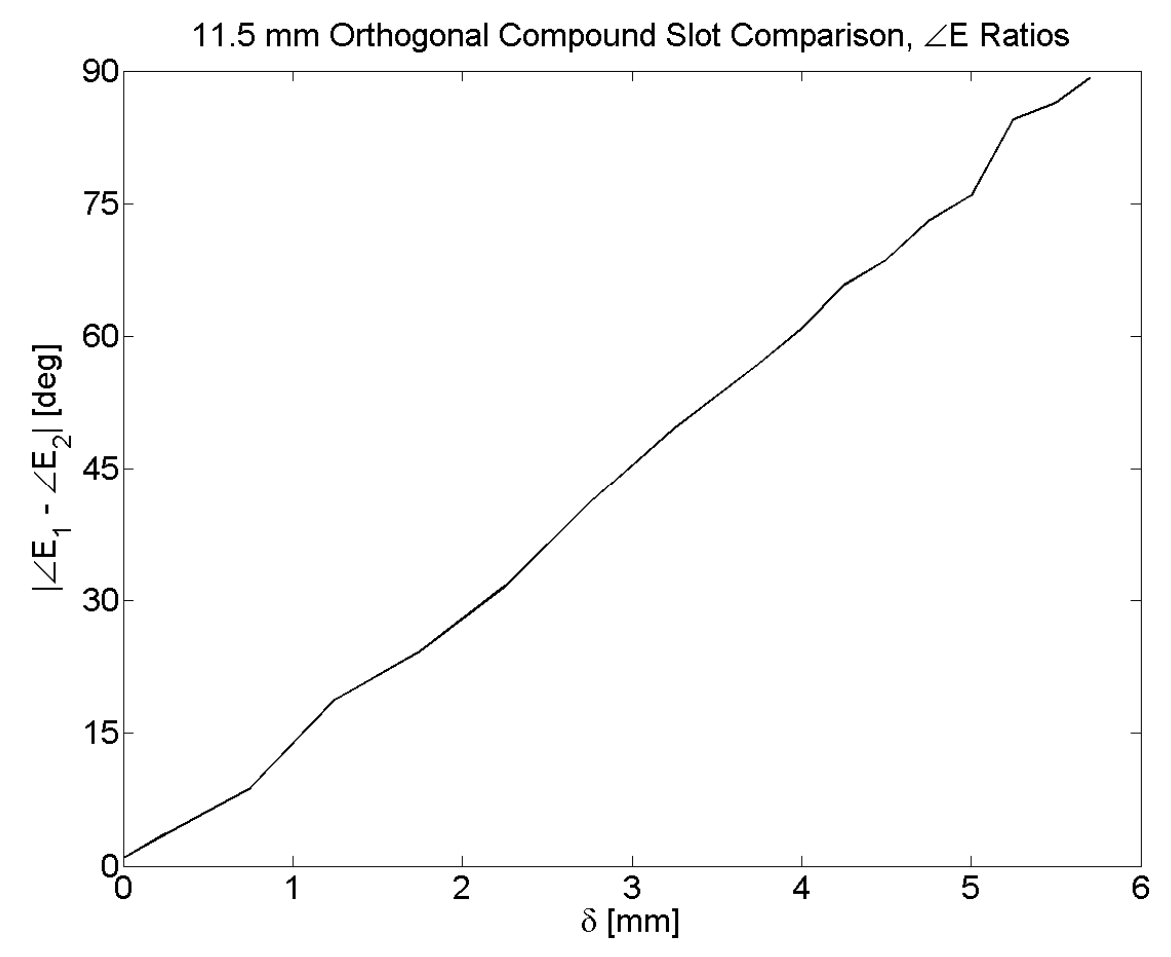

Figure 97 : Compound Slot Aperture Field Phase Comparison 
It was further theorized that if a juxtaposition of two compound, linear slots could explain the superb return loss and good axial ratio performance of the X-slot, then other slots with identical S-parameter performance should provide similar axial ratio performance, provided the dominant polarization vectors of each aperture were orthogonal. To test the theory, a single crescent slot was designed until $\mathrm{S}_{11}$ was purely reactive. A complementary slot was generated and offset until the two slot centers were coincident, per Figure 98. The crescent slot shape was simply a $90^{\circ}$ circular arc whose total length was close to the $11.5 \mathrm{~mm}$ of the compound slot, above.

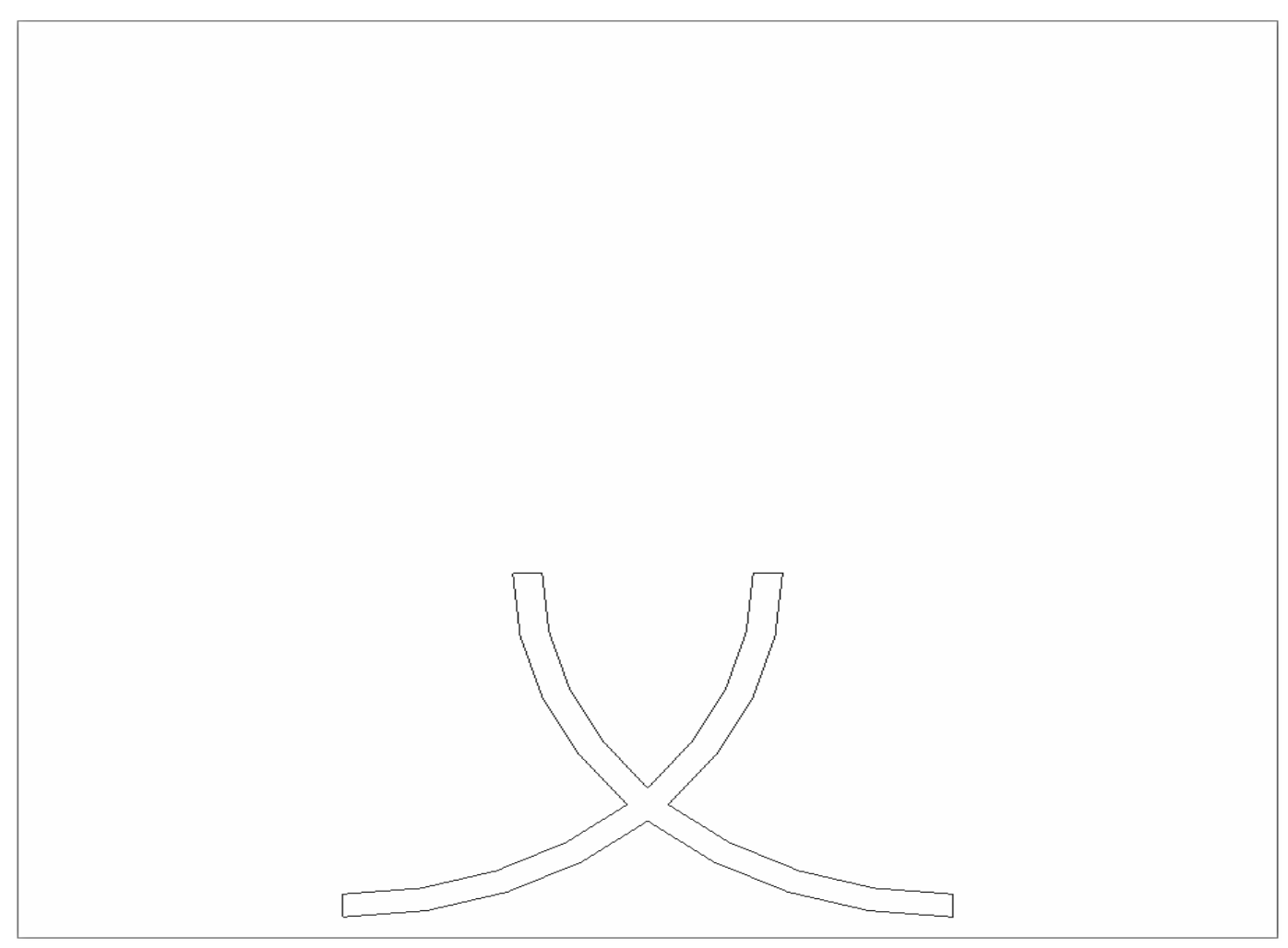

Figure 98 : Double Crescent Slot Unit Cell

The calculated S-parameter and radiation performance is captured below, in Figure 99 and Figure 100. As anticipated, both closely resemble the X-slot. Note that all color conventions follow those found in Chapters 5 and 6 . 
[S] Mag and Phase vs. Freq, $11.5 \mathrm{~mm}$ Crescent XSlot

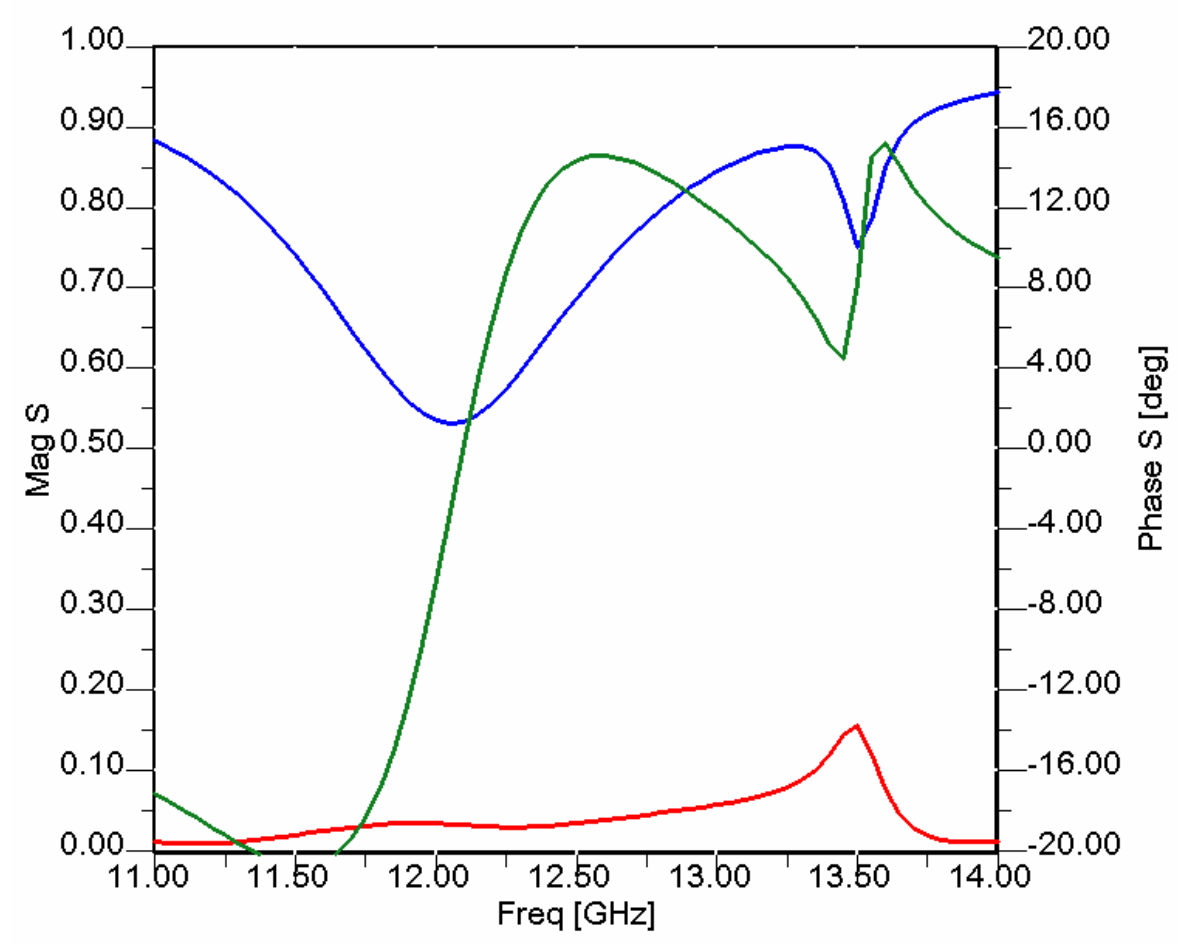

Figure 99 : DCS Unit Cell S-Parameter Performance

GP Gain \& AR vs. Theta, $11.5 \mathrm{~mm}$ Crescent XSlot

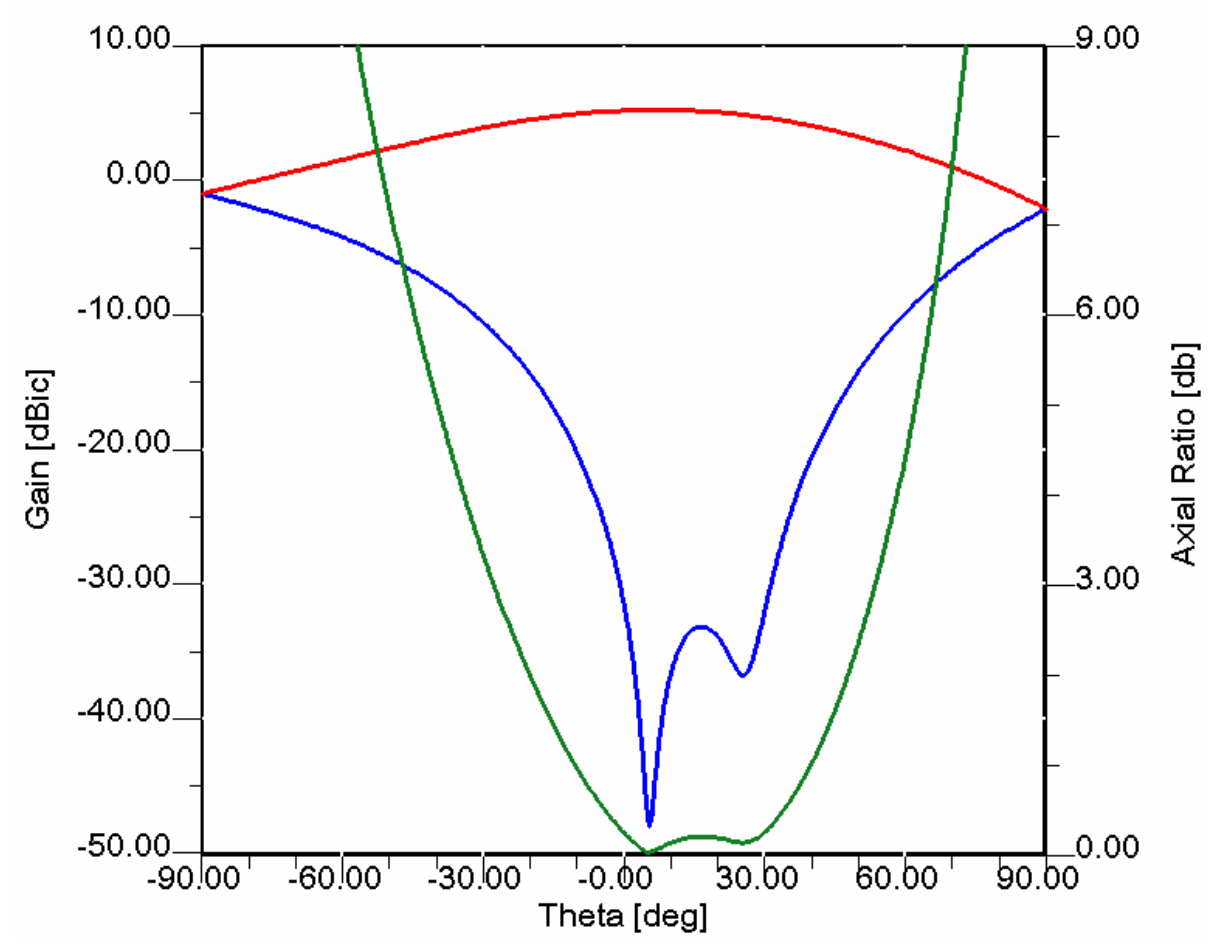

Figure 100 : DCS Unit Cell Radiation Performance 
Of particular interest in the DCS simulated data is the remarkable return loss. As with the compound slot, the single crescent slot did not exhibit very good return loss characteristics. However, at the offset where $\mathrm{S}_{11}$ became purely reactive, it is believed the two crescent slots conjugately matched one another to provide superb return loss.

The final theory of operation is not formalized beyond this point, but the design DCS design example was sufficiently successful to indicate that the relationship between $S_{11}$ and axial ratio is not unique to the X-slot. Rather, any arbitrary slot which couples equally to the transverse and longitudinal waveguide source fields will conjugately match its complement. Moreover, the aperture fields between the slot and its complement will also be in quadrature.

Further study of this topic is considered more likely to be an academic curiosity than having any practical usefulness. The study was relegated to an appendix in this work because it fell outside the primary goals set forth. 


\section{Appendix B: Derivation of Equation 13}

The goal in equation 13 is to determine the required loss tangent for a dielectrically loaded waveguide to have an identical amount of loss as an air-filled waveguide loaded with slow wave structure irises. To begin, the loss of each guide is expressed in terms of conductive and dielectric losses.

$\alpha_{s w s}=\alpha_{c}^{s w s}+\alpha_{d}^{s w s}$

$\alpha_{D L}=\alpha_{c}^{D L}+\alpha_{d}^{D L}$

In (36) and (37), $\alpha$ refers to the loss per unit length and has units of Nepers/meter. The loss terms are separated into conduction and dielectric losses, $\boldsymbol{\alpha}_{\mathrm{c}}$ and $\boldsymbol{\alpha}_{\mathbf{d}}$, respectively. The waveguide with slow wave structure loading will be assumed to have zero dielectric losses, thus the inequality from which the required loss tangent will be derived is merely:

$\alpha_{d}^{D L}<\alpha_{c}^{s w s}-\alpha_{c}^{D L}$

Each of the terms will be computed separately and compared in this form later. The first term to determine is the conduction loss of a waveguide loaded with a slow wave structure. For comparison, the conduction loss of an ordinary air-filled waveguide may be stated as follows (re-stated from (11)):

$$
\alpha=\frac{R_{s}}{b \eta \sqrt{1-\left(\frac{f_{c}}{f}\right)^{2}}}\left[1+\frac{2 b}{a}\left(\frac{f_{c}}{f}\right)^{2}\right]
$$


Through simulation of waveguides loaded with slow wave structures, it was found that the conduction loss increases directly with the slow wave number. This increase was determined to be approximately twice the ratio of the slow wave number and the empty waveguide wave number.

$$
\alpha_{c}^{s w s}=2 \frac{\beta_{s w s}}{\beta_{0}} \frac{R_{s}}{b \eta \sqrt{1-\left(\frac{f_{c}}{f}\right)^{2}}}\left[1+\frac{2 b}{a}\left(\frac{f_{c}}{f}\right)^{2}\right]
$$

For rectangular waveguide, the following relationships can be exploited.

$$
\begin{aligned}
& \beta_{0}=k_{0} \sqrt{1-\left(\frac{f_{c}}{f}\right)^{2}} \\
& f_{c}=\frac{c}{2 a \sqrt{\varepsilon_{r}}}
\end{aligned}
$$

To give this somewhat more useful form:

$$
\alpha_{c}^{s w s}=2 \frac{\beta_{s w s}}{\beta_{0}^{2}} \frac{R_{s} k_{0}}{b \eta}\left[1+\frac{b}{2 a^{3} \varepsilon_{r}}\left(\frac{c}{f}\right)^{2}\right]
$$

In (42) and (43), $\boldsymbol{\varepsilon}_{\mathbf{r}}$ is the relative dielectric constant of the medium filling the waveguide. In this case, it is equal to 1 , but the term is left for future use in the dielectric filled waveguide, to which attention is now turned.

A rectangular waveguide filled with an isotropic, homogenous dielectric

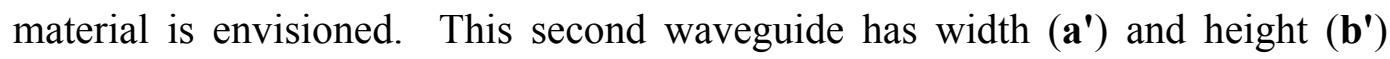
dimensions different from the waveguide above. The waveguide dimensions and 
dielectric constant of the filling medium are assumed chosen so as to permit a wave number equal to the slow wave number from above. This will permit an identical array spacing in the direction of wave propagation for both cases.

Utilizing the results from (43), the results conduction loss from the dielectrically loaded waveguide can be expressed as follows, where $\varepsilon_{\mathrm{r}}$ is used to represent the relative dielectric constant of the medium without a prime sign because it was previously equal to one and effectively unused.

$$
\alpha_{c}^{D L}=\frac{R_{s}}{b^{\prime} \eta^{\prime} \sqrt{1-\left(\frac{f_{c}^{\prime}}{f}\right)^{2}}}\left[1+\frac{b^{\prime}}{2 a^{\prime 3} \varepsilon_{r}}\left(\frac{c}{f}\right)^{2}\right]
$$

Which may be re-written as:

$$
\alpha_{c}^{D L}=\frac{R_{s} \sqrt{\varepsilon_{r}}}{b^{\prime} \eta \sqrt{1-\left(\frac{f_{c}^{\prime}}{f}\right)^{2}}}\left[1+\left(\frac{b^{\prime}}{b}\right)\left(\frac{a}{a^{\prime}}\right)^{3}\left(\frac{1}{\varepsilon_{r}}\right) \frac{b}{2 a^{3}}\left(\frac{c}{f}\right)^{2}\right]
$$

Recalling that the guided wave number of the dielectrically loaded waveguide is equal to the slow wave number of the previous structure, then (45) can be rewritten in a slightly more similar form.

$$
\beta_{0}=k_{0} \sqrt{1-\left(\frac{f_{c}^{\prime}}{f}\right)^{2}}=\beta_{s w s}
$$




$$
\alpha_{c}^{D L}=\frac{R_{s} k_{0} \sqrt{\varepsilon_{r}}}{b^{\prime} \eta \beta_{s w s}}\left[1+\left(\frac{b^{\prime}}{b}\right)\left(\frac{a}{a^{\prime}}\right)^{3}\left(\frac{1}{\varepsilon_{r}}\right) \frac{b}{2 a^{3}}\left(\frac{c}{f}\right)^{2}\right]
$$

Comparison of (47) with (43) exhibits a close similarity between the second half of each equation. To reduce the task of comparing the two equations in the inequality of (38), it was observed that the following values were typically very close to unity, making the second half of each equation very close to each other (i.e. within $10 \%$ ).

$$
\left(\frac{b^{\prime}}{b}\right)\left(\frac{a}{a^{\prime}}\right)^{3}\left(\frac{1}{\varepsilon_{r}}\right) \approx 1
$$

Again, as with the assertion of scale in (40), this could not be proven, but was observed several times in calculation. With the approximation of (46) in hand, the inequality from which the required loss tangent can be determined may be restated in expanded form.

$$
\alpha_{c}^{s w s}-\alpha_{c}^{D L} \approx\left(2 \frac{\beta_{s w s}}{\beta_{0}^{2}} \frac{R_{s} k_{0}}{b \eta}-\frac{R_{s} k_{0} \sqrt{\varepsilon_{r}}}{b^{\prime} \eta \beta_{s w s}}\right)\left[1+\frac{b}{2 a^{3}}\left(\frac{c}{f}\right)^{2}\right]
$$

This can be further factored to produce:

$$
\alpha_{c}^{s w s}-\alpha_{c}^{D L} \approx \frac{R_{s} k_{0}}{\eta \beta_{s w s}}\left(\frac{2}{b}\left(\frac{\beta_{s w s}}{\beta_{0}}\right)^{2}-\frac{\sqrt{\varepsilon_{r}}}{b^{\prime}}\right)\left[1+\frac{b}{2 a^{3}}\left(\frac{c}{f}\right)^{2}\right]
$$


Recalling (12) and re-arranging terms for a more convenient form, the following relationship can be stated about the approximate dielectric loss induced by a rectangular waveguide filled with a dielectric material characterized by a loss tangent, $\tan \boldsymbol{\delta}$.

$\alpha_{d}^{L D} \approx \tan \delta \frac{k_{0}^{2} \varepsilon_{r}}{2 \beta_{s w s}}$

Thus, via substitution of (51) and (50) into (38) and after further algebraic reduction, the following relationship may be stated for the required loss tangent for a dielectrically filled waveguide to have less overall loss than an air-filled guide with slow wave structures where both wave guides have identical wave numbers. (Note: the resistance of the side walls was replaced with the wellknown approximation for a highly conducting medium.)

$\tan \delta<\frac{1}{\varepsilon_{r}} \sqrt{\frac{2}{\omega \mu \sigma}}\left(\frac{2}{b}\left(\frac{\beta_{s w s}}{\beta_{0}}\right)^{2}-\frac{\sqrt{\varepsilon_{r}}}{b^{\prime}}\right)\left[1+\frac{b}{2 a^{3}}\left(\frac{c}{f}\right)^{2}\right]$ 


\section{Bibliography}

\section{Chapter 1}

1-1 W. H. Watson, The Physical Principles of Waveguide Transmission and Antenna Systems, Oxford, England: Clarendon, 1947

1-2 A. F. Stevenson, "Theory of Slots in Rectangular Waveguides," Journal of Applied Physics, vol. 19, pp. 24-38, Jan. 1948

1-3 A. A. Oliner, "The Impedance Properties of Narrow Radiating Slots in the Broad Face of Rectangular Waveguide," IRE Trans Ant and Prop, vol. AP-5, pp. 4-20, Jan. 1957

1-4 R. S. Elliot \& L. A. Kurtz, "The Design of Small Slot Arrays," IEEE Trans Ant and Prop, vol. AP-26, pp. 214-219, Mar. 1978

1-5 R. S. Elliot, "On the Design of Traveling-Wave-Fed Longitudinal Shunt Slot Arrays," IEEE Trans Ant and Prop, vol. AP-27, pp. 717-720, Sept. 1979

1-6 R. S. Elliot, Antenna Theory and Design, Englewood Cliffs, NJ: PrenticeHall, 1981, sec. $8.13-8.17$

1-7 R. S. Elliot, "An Improved Design Procedure for Small Arrays of Shunt Slots", IEEE Trans Ant and Prop, vol. AP-31, pp. 48-53, Jan. 1983

1-8 G. J. Stern and R. S. Elliot, "Resonant Length of Longitudinal Slots and Validity of Circuit Representation: Theory and Experiment," IEEE Trans Ant and Prop, vol. AP-33, pp. 1264-1271, Nov. 1985

1-9 R. C. Hansen, Microwave Scanning Antennas, Peninsular, Tallahassee, FL, 1986

1-10 A. Dion, "Nonresonant Slotted Arrays," IRE Trans Ant and Prop, vol. AP-6, pp. 360-365, Oct. 1958

1-11 T. V. Khac, "A Study of Some Slot Discontinuities in Rectangular Waveguides," Ph.D. dissertation, Monash University, Australia, 1974

1-12 B. J. Maxum, "Resonant Slots with Independent Control of Amplitude and Phase," IRE Trans Ant and Prop, vol. AP-8, pp. 384-388, July 1960 
1-13 S. R. Rengarajan, "Compound Radiating Slots in a Broad Wall of a Rectangular Waveguide," IEEE Trans Ant and Prop, vol. AP-37, pp. 11161123, Sept. 1989

1-14 IBID, Sec. 6.9

1-15 A. J. Simmons, "Circularly Polarized Slot Radiators," IRE Trans Ant and Prop, vol. AP-5, pp. 31-36, Jan. 1957

1-16 J. Hirokawa, "A Study of Slotted Waveguide Array Antennas," Ph.D. dissertation, Tokyo Institute of Technology, Japan, 1993

1-17 J. Hirokawa, et al., "A Single Layer Slotted Leaky Waveguide Array Antenna for Mobile Reception of Direct Broadcast from Satellite," IEEE Trans Vehicular Tech, vol. 44, pp. 749-755, Nov. 1995

1-18 T. Hirano, et al., "Waveguide Matching Crossed-Slot," IEE Proc - Micro, Ant, and Prop, vol. 150, pp. 143-146, June 2003

1-19 N. Goto and M. Yamamoto, "Circularly Polarized Radial Line Slot Antennas,” Tech. Rep. AP80-57, IEICE, Japan, Aug. 1980

1-20 A Akiyama, et al., "Numerical Optimisation (sic) of Slot Parameters for a Concentric Array Radial Line Slot Antenna," IEE Proc - Micro, Ant, and Prop, vol. 145, pp. 141-145, April 1998

1-21 M. Ando, et al., "Novel Single-Layer Waveguides for High-Efficiency Millimeter-Wave Arrays," IEEE Trans Micro Theory and Tech, vol. 46, pp. 792-799, June 1998

\section{Chapter 2}

2-1 K. Sakakibara, et al., "A Two-Beam Slotted Leaky Waveguide Array for Mobile Reception of Dual-Polarization DBS," IEEE Trans Ant and Prop, vol. AP-48, pp. 1-7, Jan. 1999

2-2 M. Takahashi, et al., "Dual Circularly Polarized Radial Line Slot Antennas," IEEE Trans Ant and Prop, vol. AP-43, pp. 874-876, Aug. 1995

2-3 S. Park, et al., "A Slotted Post-Wall Waveguide Array with Interdigital Structure for $45^{\circ}$ Linear and Dual Polarization," IEEE Trans Ant and Prop, vol. AP-53, pp. 2865-2871, Sept. 2005 
2-4 N. F. Sali, et al., "Impedance Properties of X-Slot Array as a Function of Displacement on Broad Face of Rectangular Waveguide," IEEE Proc of Aero and Elec Conf, vol. 1, pp. 316-319, May 1992

2-5 R. S. Elliott, “On the Theory of Corrugated Plane Surfaces," IRE Trans Ant and Prop, vol. AP-2, pp. 71-81, April 1954

2-6 R. E. Collin, Foundations for Microwave Engineering $2^{\text {nd }}$ ed., McGraw-Hill, N.Y., 1992

2-7 D. M. Pozar, Microwave Engineering $2^{\text {nd }}$ ed., Wiley, N.Y., 1998, Sec. 8.1

2-8 Ansoft Corporation, Pittsburgh, PA (www.ansoft.com)

2-9 CST of America, Inc., Wellesley, MA (www.cst.com)

2-10 EM Software \& Systems, Stellenbosch, South Africa (www.feko.info)

2-11 A. F. Seaton and G. A. Carnegis, "A Novel Circularly Polarized Planar Array for Surveyor,” IRE Int'l Conv Rec, vol. 11, pp. 2-9, Mar. 1963

2-12 A. J. Sangster, “Circularly Polarized Linear Waveguide Array,” IEEE Trans Ant and Prop, vol. AP-21, pp. 704-705, Sept. 1973

2-13 J. Hirokawa, "Analysis of a Slot Pair on a Corrugated Waveguide of Finite Length and Extraction of its Coupling Factor," Proc URSI Radio Science Mtg, vol. 1, pg. 612, July 2006

2-14 X. Shan, “A Novel Waveguide Transverse Slot Antenna Array,” IEEE Proc - Ant and Prop, vol. 3, pp.420-423, June 2002

\section{Chapter 3}

3-1 L. Josefsson, "A Waveguide Transverse Slot for Array Applications," IEEE Trans Ant and Prop, vol. AP-41, pp. 845-850, July 1993

3-2 G. Montisci, et al., "A Polarization-agile Waveguide Slot Antenna," IEEE Proc - Ant and Prop, vol. 3, pp. 1034-1037, June 2003

3-3 G. Montisci, et al., "Waveguide Slot Antennas for Circularly Polarized Radiated Field," IEEE Trans Ant and Prop, vol. AP-52, pp. 619-623, Feb. 2004

3-4 C. A. Balanis, Advanced Engineering Electromagnetics, Wiley, N.Y., 1989, Sec. $8.1-8.5$ 
3-5 J. Dittloff, et al., "Optimum Design of Waveguide E-Plane Stub-Loaded Phase Shifters," IEEE Trans Ant and Prop, vol. AP-36, pp. 582-587, March 1988

3-6 I. L. Verbitskii, "Dispersion Relations for Comb-Type Slow-Wave Structures," IEEE Micro Theory and Tech, vol. MTT-28, pp. 48-50, Jan. 1980

3-7 J. Jobert and D. A. McNamara, "Analysis of Radiating Slots in a Rectangular Waveguide Inhomogenously Loaded with a Dielectric Slab", IEEE Trans Ant and Prop, vol. AP-41, pp. 1212-1221, Sept. 1993

3-8 National Magnetics Group, Bethlehem, PA (www.magneticsgroup.com)

3-9 R. C. Johnson, Antenna Engineering Handbook, McGraw-Hill, N.Y., 1992, Chap. 9

3-10 R. C. Hansen, Phased Array Antennas, Wiley, N.Y., 1998, Sec. 5.2 \& 6.1.2

\section{Chapter 4}

4-1 O. F. Siddiqui, et al., "Periodically Loaded Transmission Line With Effective Negative Refractive Index and Negative Group Velocity," IEEE Trans Ant and Prop, vol. AP-51, pp. 2619-2625, Oct. 2003

4-2 O. F. Siddiqui, et al., "Time-Domain Measurement of Negative Group Delay in Negative-Refractive-Index Transmission-Line Metamaterials," IEEE Micro Theory and Tech, vol. MTT-52, pp. 1449-1454, May 2004

4-3 R. E. Collin, Field Theory of Guided Waves $2^{\text {nd }}$ ed., Wiley-IEEE, N.Y., 1990, Chap. 9

4-4 N. Marcuvitz, Waveguide Handbook, MIT Rad Lab Series, vol. 10, McGraw-Hill, N.Y., 1951

4-5 The MathWorks, Inc., Natick, MA (www.mathworks.com)

4-6 Microwave Innovation Group, Bremen, Germany (www.mig-germany.com)

4-7 M. J. D. Powell, "An Efficient Method for Finding the Minimum of a Function of Several Variables Without Calculating Derivatives," Computer Journal, vol. 7, pp. 155-162, July 1964 
4-8 J. W. Bandler, "Optimization Methods for Computer-Aided Design," IEEE Micro Theory and Tech, vol. MTT-17, pp. 533-552, Aug. 1969

\section{Chapter 6}

6-1 R. J. Mailloux, Phased Array Antenna Handbook $2^{\text {nd }}$ ed., Artech House, Boston, Sec. 8.3 